\title{
Struktureigenschaften und molekulare Dynamik flüssiger Ethylenglykol-Oligomere und ihrer Mischungen mit Wasser
}

\author{
Dissertation \\ zur Erlangung des Doktorgrades \\ der Mathematisch-Naturwissenschaftlichen Fakultäten \\ der Georg-August-Universität zu Göttingen
}

\author{
vorgelegt von \\ Elke Hanke \\ aus Hildesheim
}

Göttingen 2007 
D7

Referent: Prof. Dr. Dirk Ronneberger

Korreferent: Priv.-Doz. Dr. Christian Jooß

Tag der mündlichen Prüfung: 3. Juli 2007 


\section{Inhaltsverzeichnis}

1 Einleitung 1

2 Die verwendeten Substanzen 5

2.1 Präparation und Behandlung der Messproben . . . . . . . . . 5

3 Ultraschallspektroskopie 9

3.1 Schallabsorption in Flüssigkeiten $\ldots \ldots \ldots \ldots \ldots$

3.1.1 Relaxation thermischer Gleichgewichte . . . . . . . . 10

3.2 Messverfahren . . . . . . . . . . . . . . . . . . . . . . . 12

3.2 .1 Resonatorverfahren . . . . . . . . . . . . . . . . . 13

3.2.2 Schwingungspulstransmissionsverfahren . . . . . . . . 25

4 Ultraschallabsorptionsspektren der Oligoethylenglykole 35

4.1 Auswertung der Resonatordaten gegen unterschiedliche Referenzen . 37

4.2 Analytische Beschreibung der Oligoethylenglykolspektren . . . . . . 42

4.2 .1 Anpassungsalgorithmus . . . . . . . . . . . . . . . . . . . 42

4.2 .2 Dispersion . . . . . . . . . . . . . . . . . . . . . 42

4.2.3 Beschreibung durch Debye-Spektralfunktionen . . . . . . . . 44

4.2 .4 Beschreibung durch andere Spektralfunktionen . . . . . . . 47

4.3 Diskussion der Ergebnisse . . . . . . . . . . . . . . . . . 47

4.3.1 Rouse-Modell und Modell gedämpfter Torsionsoszillatoren . 48

4.3.2 Beschreibung der Spektren auf Grundlage des Rouse-/DTOModells . . . . . . . . . . . . . . . . . . 52

4.3.3 Verhältnis von Scher- und Volumenviskosität bei tiefen Frequenzen . . . . . . . . . . . . . . 76

4.3.4 Temperaturabhängigkeit von Relaxationszeit und -amplitude 77

5 Dielektrische Spektroskopie $\quad 79$

5.1 Grundlagen . . . . . . . . . . . . . . . . . . . . . . . . . . 79

5.1 .1 Dielektrische Relaxation . . . . . . . . . . . . . . . 79

5.1 .2 Leitfähigkeit . . . . . . . . . . . . . . . . . . . . . 80

5.2 Messverfahren . . . . . . . . . . . . . . . . . . . . . 81

5.2 .1 Messprinzip . . . . . . . . . . . . . . . . . . . 81

$5.2 .2 \quad$ Fehler des Verfahrens . . . . . . . . . . . . . . . . . 84 
6 Die Ergebnisse der dielektrischen Spektroskopie 87

6.1 Analytische Beschreibung . . . . . . . . . . . . . . . 87

6.2 Diskussion der Ergebnisse . . . . . . . . . . . . . . . . . . . 87

6.2 .1 Der Verteilungsparameter … . . . . . . . . . 87

6.2.2 Die Hauptrelaxationszeit der Cole-Cole-Spektralfunktion . . 92

6.2.3 Die zu tiefen Frequenzen hin extrapolierte Dielektrizitätszahl 100

6.2.4 Abschätzung der Dipolorientierungskorrelation . . . . . . . 100

7 Beeinflussung der Kettendynamik durch Wasserstoffbrückenasso$\begin{array}{ll}\text { ziation } & 107\end{array}$

7.1 PEG-Schmelzen . . . . . . . . . . . . . . . . . . . 107

7.2 PEG/Wasser-Mischungen . . . . . . . . . . . . . . 109

7.2.1 Konzentrationsabhängiger Verlauf der Relaxationzeiten . . . 112

8 Zusammenfassung und Ausblick 115

9 Anhang 119

9.1 Ergänzende Dichte-, Schallgeschwindigkeits- und Viskositätsmessungen . . . . . . . . . . . . . . . . . . . . . . . 119

$\begin{array}{ll}\text { Literaturverzeichnis } & 123\end{array}$

$\begin{array}{ll}\text { Danksagung } & 129\end{array}$

$\begin{array}{ll}\text { Curriculum vitae } & 131\end{array}$ 


\section{Einleitung}

Die Polymerphysik hat sich in den vergangenen Jahrzehnten zu einem weiten Forschungsfeld entwickelt. Dabei besteht nicht nur ein grundlegendes Interesse an den zahlreichen ungewöhnlichen physikalischen und chemischen Eigenschaften polymerer Substanzen. Die genaue Kenntnis beispielsweise des Zusammenhangs von mikroskopischer Struktur und makroskopischem Verhalten spielt auch bei der Entwicklung neuer Materialien für Industrie und Technik eine große Rolle. Daneben kommen viele Erkenntnisse über strukturelle und dynamische Eigenschaften von synthetischen Polymeren und Biopolymeren in der Pharmazie und Medizin zur Anwendung [1, 2].

Wesentlich für die Eigenschaften polymerer Stoffe ist die Dynamik der Polymerketten. Sie soll im Rahmen dieser Arbeit an Schmelzen kurzkettiger linearer Polymere hauptsächlich mit Hilfe der Ultraschallabsorptionsspektroskopie untersucht werden. Mit dieser Methode können schnelle molekulare Prozesse in Flüssigkeiten detektiert werden, die mit Änderungen des molekularen Volumens bzw. der Enthalpie des Systems verbunden sind. Darunter fallen auch Konformationsänderungen, wie sie der Polymerkettenbewegung zugrunde liegen.

Frequenzabhängige Messungen des Schallabsorptionskoeffizienten an Polymerschmelzen, -mischungen und -lösungen wurden in den letzten Jahren von verschiedenen Arbeitsgruppen veröffentlicht [3-5]. Dabei stand insbesondere die Untersuchung des kritischen Entmischungsverhaltens von binären Polymer/PolymerSystemen und Polymerlösungen im Vordergrund [3, 5]. Durch den nur schmalen verfügbaren Frequenzbereich (für die genannten Veröffentlichungen liegt er im Intervall 0.2-90 MHz) wird die Interpretation der sich über mehrere Dekaden erstreckenden Absorptionsspektren sehr erschwert. Eine detaillierte Analyse der Spektren im Hinblick auf die Kettendynamik war daher kaum möglich. Insbesondere bei Mischungen ist die genaue Kenntnis der Spektren der reinen Polymere jedoch von großer Bedeutung, da die Eigenschaften der Mischungen durch die der reinen Substanzen wesentlich mitbestimmt werden.

Die in dieser Arbeit vorgestellten Messungen lassen aus zwei Gründen einen tieferen Aufschluss über die Kettendynamik erwarten: Zum einen steht ein zu hohen Frequenzen deutlich erweiterter Messbereich zur Verfügung (0.2 bis $2000 \mathrm{MHz}$ ). Zum anderen werden die komplizierten und breiten Absorptionsspektren systematisch vereinfacht, indem die Zahl der Bewegungsfreiheitsgrade durch die Wahl kleiner Polymerisationsgrade und gezielte, schrittweise Verkürzung der Ketten verringert wird. Überprüft werden soll, inwieweit die so erhaltenen Absorptionsspektren auf der Grundlage einfacher Theorien für die Kettendynamik (Rouse [6], Tobolsky et al. [7, 8]) analysiert werden können und die Beschreibung der Oligomerketten- 
bewegung mit diesen Modellen möglich ist.

In der vorliegenden Arbeit wird hauptsächlich das Polymer Polyethylenglykol (PEG) untersucht. Die vielseitigen Eigenschaften dieses Polymers spiegeln sich in der Breite seiner industriellen Anwendungen wider: So sind beispielsweise kurze PEG-Ketten in zahlreichen kosmetischen Produkten als Weichmacher, Feuchthaltemittel oder Schaumbildner enthalten [9, 10], wohingegen geringe Mengen langkettiger Polyethylenglykole eingesetzt werden, um den Strömungswiderstand in wasserdurchflossenen Leitungen und Röhren herabzusetzen [11, 12]. Vielversprechend sind Entwicklungen im biotechnologischen und medizinischen Bereich: Bei verschiedenen biologisch relevanten Molekülen, die mit Polyethylenglykolen chemisch verknüpft wurden, konnte eine Erhöhung der Löslichkeit sowie ihrer Lebensdauer im Blutkreislauf beobachtet werden [1]. Auch von einer reduzierten Immunogenizität und Antigenizität PEG-modifizierter Proteine wird berichtet [1]. Eine weitere interessante Anwendung ist die chemische Modifikation von Oberflächen durch PEG-Moleküle, durch die die Anlagerung von Proteinen und Zellen verhindert werden kann [1]. Dieser Effekt wird beispielsweise bei der Konservierung archäologischer Funde ausgenutzt [13].

\section{$\mathrm{HO}-\mathrm{CH}_{2} \varlimsup_{-\mathrm{CH}_{2}}-\mathrm{O}-\mathrm{CH}_{2} \ddagger_{\mathrm{n}-1} \mathrm{CH}_{2}-\mathrm{OH}$}

Abbildung 1.1: Chemische Struktur von Polyethylenglykol

Wegen seiner einfachen linearen und flexiblen Struktur (Abb. 1.1) ist Polyethylenglykol sehr gut als Modellsystem für diese Arbeit geeignet. Eine weitere nützliche Eigenschaft ist seine hervorragende Löslichkeit in Wasser [14-16]. Sie stellt insofern eine Besonderheit dar, als die strukturell ähnlichen Polyether Polymethylenglykol $\left(\mathrm{H}-\left[\mathrm{OCH}_{2}\right]_{n}-\mathrm{OH}\right)$ bzw. Polytrimethylenglykol $\left(\mathrm{H}-\left[\mathrm{OCH}_{2} \mathrm{CH}_{2} \mathrm{CH}_{2}\right]_{n}-\mathrm{OH}\right)$ nicht wasserlöslich sind [14], obwohl in ersterem die Konzentration hydrophiler Gruppen deutlich höher ist als bei PEG. Dieses unerwartete Lösungsverhalten wird in einigen Arbeiten darauf zurückgeführt, dass der Abstand benachbarter Sauerstoffatome in flüssigem Polyethylenglykol etwa dem Abstand der Wasserstoffatome eines Wassermoleküls entspricht, wodurch sich die PEG-Kette besser in das Wasserstoffbrückennetzwerk des Wassers einfügt als seine chemischen Nachbarn. In diesem Zusammenhang wird auch eine Stabilisierung der Polymerstruktur bzw. die Ausbildung einer Helixstruktur in wässrigen Lösungen durch eine umgebende Hydrathülle diskutiert [17-19].

In der vorliegenden Arbeit wird die Wasserlöslichkeit von PEG ausgenutzt, um den Einfluss der Wasserstoffbrückenassoziation auf die Kettendynamik zu untersuchen. Dazu werden frequenzabhängige Messungen der komplexen Dielektrizitätszahl an PEG/Wasser-Mischungen mit unterschiedlichen Mischungsverhältnissen durchgeführt, die Aussagen über die Beweglichkeit und Orientierung der dipolaren $\mathrm{CH}_{2}$-O-CH${ }_{2}$-Gruppen in der PEG-Kette erlauben. Durch Variation des Wasseranteils sowie der Polymerkettenlänge und -endgruppen soll dabei der Assoziations- 
grad systematisch verändert werden. Inwieweit die Beweglichkeit der $\mathrm{CH}_{2}-\mathrm{O}-\mathrm{CH}_{2}{ }^{-}$ Gruppen auch die Flexibilität der gesamten Polymerkette beeinflusst, soll durch einen Vergleich von dielektrischen und akustischen Spektren ermittelt werden. Zur Ergänzung der Ergebnisse aus der dielektrischen und akustischen Spektroskopie werden an den untersuchten Lösungen und Schmelzen Messungen der Schallgeschwindigkeit, der Dichte und der Viskosität vorgenommen. 
1 Einleitung 


\section{Die verwendeten Substanzen}

Alle verwendeten Oligomere wurden von der Firma Aldrich bezogen. Sie sind in den Tabellen 2.1 und 2.2 zusammen mit ihrem Reinheitsgrad aufgeführt. Außerdem finden sich dort Angaben zum Molekulargewicht und der sich daraus ergebenden Kettenlänge.

Ab einer Kettenlänge von vier monomeren Einheiten ist das Molekulargewicht keine wohldefinierte Größe mehr, sondern unterliegt einer Verteilung. Der Hersteller gibt in diesem Fall ein mittleres Molekulargewicht $\bar{M}$ oder die maximale Breite der Verteilung an. Zusätzlich liegen zu den meisten Polymeren Analysenzertifikate mit genaueren Angaben über das anzahlgemittelte Molekulargewicht $\bar{M}_{\text {mess }}$ vor. Je nach Produktionscharge variiert $\bar{M}_{\text {mess }}$, weicht aber bei den verwendeten Schmelzen weniger als $3 \%$ vom Sollwert ab. Einzige Ausnahme bildet Polyethylenglykoldimethylether 250. Hier liegt $\bar{M}_{\text {mess }}$ für die von uns verwendete Charge um $14 \%$ über dem Sollwert $250 \mathrm{~g} / \mathrm{mol}$. Die mittlere Kettenlänge $\bar{n}$, d.h. die mittlere Anzahl monomerer Einheiten pro Molekül, wurde für alle Polymere aus dem chargenunspezifischen mittleren Molekulargewicht $\bar{M}$ errechnet. Eine Berechnung aus dem chargenabhängigen Messwert $\bar{M}_{\text {mess }}$ würde außer im Fall von PEGDME 250 nur auf geringfügig andere Kettenlängen führen. Da sich auch die Parameter der Ultraschallspektren für unterschiedliche Herstellungsreihen nur innerhalb der Messfehler ändern, wird der hierdurch gemachte Fehler als vernachlässigbar angesehen. Die größere Abweichung zwischen dem Soll- und Ist-Wert bei PEGDME 250 wird bei der Auswertung der Daten entsprechend berücksichtigt. Auch der Fehler im absoluten Molekulargewicht, der vom Hersteller für PEG 300, PEG 400 und PEG 600 mit $\pm 5 \%$ angesetzt ist, wird in die Auswertung miteinbezogen.

Substanzen, die zur Herstellung von Referenzlösungen für die Ultraschallspektroskopie verwendet wurden, sind in Tabelle 2.3 aufgelistet.

\subsection{Präparation und Behandlung der Messproben}

Die Chemikalien wurden, wie vom Hersteller geliefert, ohne weitere Reinigung oder Trocknung verwendet. Um eine Erhöhung des Wassergehalts der hygroskopischen Polymerschmelzen zu vermeiden, wurden die Flaschen nach dem ersten Öffnen im Exsikkator über einer Schicht Trockenmittel (Silicagel-Perlen) aufbewahrt. Proben aus den Originalflaschen wurden ausschließlich unter Stickstoffatmosphäre entnommen. Die Ultraschallmessungen der unverdünnten Polymerschmelzen fanden unter ständiger Spülung mit trockenem Stickstoff statt. Zusätzlich wurde die Atmosphäre im Thermostatiergehäuse, in dem sich die Messzelle zur thermischen Abschirmung 


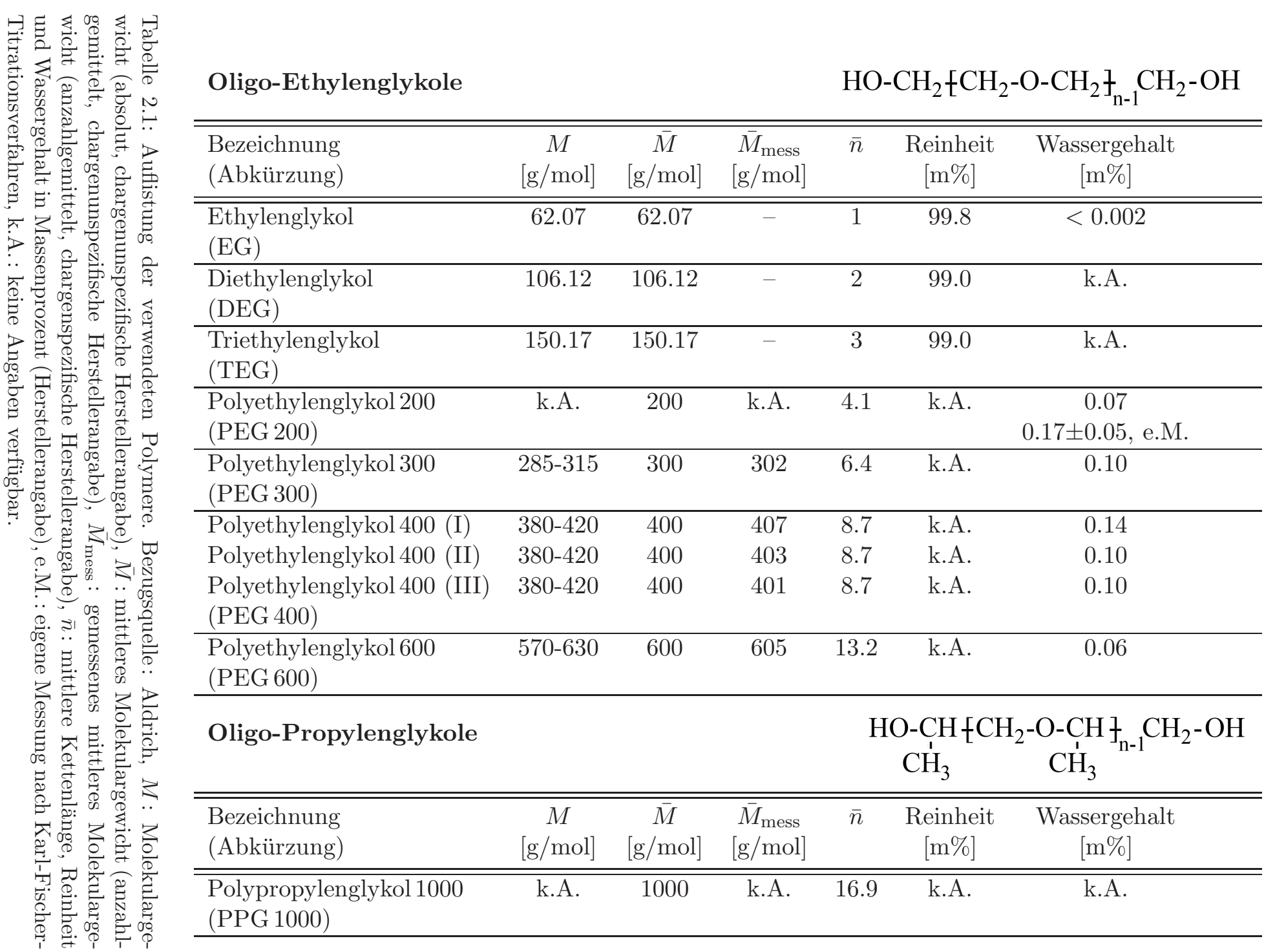


Dimethylether-Derivate der Ethylenglykol-Oligomere $\quad \mathrm{H}_{3} \mathrm{C}-\mathrm{O}-\mathrm{CH}_{2} \mathrm{fCH}_{2}-\mathrm{O}-\mathrm{CH}_{2} \mathrm{f}_{\mathrm{n}-\mathrm{l}} \mathrm{CH}_{2}-\mathrm{O}-\mathrm{CH}_{3}$

\begin{tabular}{|c|c|c|c|c|c|c|}
\hline $\begin{array}{l}\text { Bezeichnung } \\
\text { (Abkürzung) }\end{array}$ & $\begin{array}{c}M \\
{[\mathrm{~g} / \mathrm{mol}]}\end{array}$ & $\begin{array}{c}\bar{M} \\
{[\mathrm{~g} / \mathrm{mol}]}\end{array}$ & $\begin{array}{c}\bar{M}_{\text {mess }} \\
{[\mathrm{g} / \mathrm{mol}]}\end{array}$ & $\bar{n}$ & $\begin{array}{c}\text { Reinheit } \\
\text { [m\%] }\end{array}$ & $\begin{array}{l}\text { Wassergehalt } \\
{[\mathrm{m} \%]}\end{array}$ \\
\hline $\begin{array}{l}\text { Triethylenglykoldimethylether } \\
\text { (TEGDME) }\end{array}$ & 178.23 & 178.23 & k.A. & 3 & $\overline{99.0}$ & k.A. \\
\hline $\begin{array}{l}\text { Polyethylenglykoldimethylether } 250 \\
\text { (PEGDME 250) }\end{array}$ & k.A. & 250 & 284 & 4.6 & k.A. & $\begin{array}{c}0.29 \\
0.36 \pm 0.05, \text { e.M. }\end{array}$ \\
\hline $\begin{array}{l}\text { Polyethylenglykoldimethylether } 500 \\
\text { (PEGDME 500) }\end{array}$ & k.A. & 500 & 486 & 10.3 & k.A. & $0.33 \pm 0.05$, e.M. \\
\hline \multicolumn{5}{|c|}{ Monoethylether-Derivate der Ethylenglykol-Oligomere } & \multicolumn{2}{|r|}{$\mathrm{H}_{3} \mathrm{C} f \mathrm{CH}_{2}-\mathrm{O}-\mathrm{CH}_{2} \mathrm{f}_{\mathrm{n}} \mathrm{CH}_{2}-\mathrm{OH}$} \\
\hline $\begin{array}{l}\text { Bezeichnung } \\
\text { (Abkürzung) }\end{array}$ & $\begin{array}{c}M \\
{[\mathrm{~g} / \mathrm{mol}]}\end{array}$ & $\begin{array}{c}\bar{M} \\
{[\mathrm{~g} / \mathrm{mol}]}\end{array}$ & $\begin{array}{c}\bar{M}_{\text {mess }} \\
{[\mathrm{g} / \mathrm{mol}]}\end{array}$ & $\bar{n}$ & $\begin{array}{c}\text { Reinheit } \\
\text { [m\%] }\end{array}$ & $\begin{array}{l}\text { Wassergehalt } \\
{[\mathrm{m} \%]}\end{array}$ \\
\hline $\begin{array}{l}\text { Triethylenglykolmonoethylether } \\
\text { (TEGMEE) }\end{array}$ & 1788.23 & 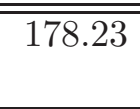 & k.A. & 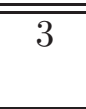 & k.A. & 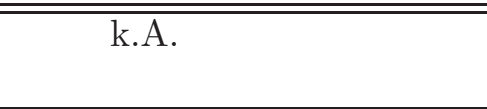 \\
\hline
\end{tabular}




\begin{tabular}{lcccc}
\hline \hline Bezeichnung & $\begin{array}{c}M \\
{[\mathrm{~g} / \mathrm{mol}]}\end{array}$ & Strukturformel & Bezugsquelle & $\begin{array}{c}\text { Reinheit } \\
{[\mathrm{m} \%]}\end{array}$ \\
\hline \hline Ethanol & 46.07 & $\mathrm{H}_{3} \mathrm{C}-\mathrm{CH}_{2}-\mathrm{OH}$ & $\begin{array}{c}\text { Riedel de Haën/ } \\
\text { Scharlau }\end{array}$ & 99.8 \\
\hline Harnstoff & 60.06 & $\mathrm{O}=\mathrm{C}-\mathrm{NH}_{2}$ & Fluka & $\geq 99.5$ \\
\hline
\end{tabular}

Tabelle 2.3: Ausgangssubstanzen der Referenzlösungen. Bezeichnungen wie in Tab. 2.1.

befindet, durch Trockenmittel wasserfrei gehalten. Messproben reiner Polymerschmelzen wurden erst am Tag der Messung abgefüllt und kein zweites Mal verwendet. Das zum Ansetzen der Lösungen benötigte Wasser wurde zuvor in einem mehrstufigen Millipore-Ionenaustauscher deionisiert und gereinigt. Die Einwaage der Chemikalien erfolgte auf Analysewaagen mit einer Genauigkeit von $\pm 0.01 \mathrm{~g}$ (Sartorius BP $3100 \mathrm{~S}$ und Sartorius Typ 2258). Da niemals weniger als $1.5 \mathrm{~g}$ einer Substanz abgewogen wurde, liegt der Fehler im Massenanteil damit bei maximal $0.7 \%$. Alle Messsubstanzen wurden stets in gut verschließbaren Gefäßen (Erlenmeyerkolben/Deckelgläsern) aufbewahrt und zusätzlich mit Parafilm abgedichtet. Vor jeder Messung wurden die Proben im Vakuumofen bzw. im Ultraschallbad entgast. 


\section{Ultraschallspektroskopie}

\subsection{Schallabsorption in Flüssigkeiten}

Aus den Erhaltungssätzen der Kontinuumsmechanik für den Impuls (Navier-StokesGleichung) und die Masse (Kontinuitätsgleichung) kann man in adiabatischer Näherung eine akustische Wellengleichung aufstellen, deren Lösungen allgemein die Schallausbreitung in einer homogenen viskosen Flüssigkeit beschreiben. Durch Lösung dieser Wellengleichung für den Schalldruck $p$ mit einem harmonischen Ansatz

$$
p(x, t)=\hat{p} e^{i \omega t-\gamma x}
$$

erhält man für die komplexe Ausbreitungskonstante $\gamma=\alpha+i \beta$ einer in positiver $x$-Richtung laufenden ebenen Schallwelle der Frequenz $f$ und Wellenlänge $\lambda$ die Beziehung [20]

$$
\gamma^{2}=\frac{-\omega^{2} \rho}{K+\frac{4}{3} i \omega \eta_{\mathrm{s}}}
$$

mit

$\begin{array}{ll}t & : \text { Zeit } \\ \omega=2 \pi f & : \text { Kreisfrequenz } \\ \alpha & : \text { Absorptionskoeffizient } \\ \beta=2 \pi / \lambda & : \text { Wellenzahl } \\ K=\kappa_{\mathrm{s}}^{-1}+i \omega \eta_{\mathrm{v}} & : \text { komplexer adiabatischer Kompressionsmodul } \\ \kappa_{\mathrm{s}}=\left(\rho c_{\mathrm{s}}^{2}\right)^{-1} & : \text { adiabatische Kompressibilität } \\ c_{\mathrm{s}}=\lambda f & : \text { Wellenausbreitungsgeschwindigkeit } \\ \rho & : \text { Dichte } \\ \eta_{\mathrm{s}} & : \text { Scherviskosität } \\ \eta_{\mathrm{v}} & : \text { Volumenviskosität. }\end{array}$

Aus (3.2) ergibt sich ein Absorptionskoeffizient

$$
\alpha(f)=\frac{2 \pi^{2}}{\rho c_{\mathrm{s}}^{3}}\left(\frac{4}{3} \eta_{\mathrm{s}}+\eta_{\mathrm{v}}\right) \cdot f^{2},
$$

der den Amplitudenabfall einer Schallwelle entlang ihrer Ausbreitungsrichtung beschreibt und die zentrale Messgröße der akustischen Spektroskopie darstellt. Die Scherviskosität $\eta_{\mathrm{s}}$ spiegelt dabei die Verluste bei Scherung eines Flüssigkeitselements wider, die Volumenviskosität den Anteil am Absorptionskoeffizienten, der 
die Verluste bei allseitiger Kompression eines Flüssigkeitsvolumens beschreibt. Historisch bedingt werden hierbei die Scherviskosität und/oder die Volumenviskosität in der Literatur häufig als frequenzunabhängig angenommen [20-23] und der durch sie beschriebene Beitrag zur Ultraschalldämpfung nur als Hintergrundsanteil einer Gesamtabsorption betrachtet. Tatsächlich sind jedoch beide Größen frequenzabhängig und es können sowohl Scher- als auch Volumenrelaxationsprozesse im betrachteten Frequenzbereich liegen [24]. Dies ist bei den untersuchten Substanzen der Fall. Gleichung (3.3) ist also zu lesen als

$$
\alpha(f)=\frac{2 \pi^{2}}{\rho c_{\mathrm{s}}^{3}}\left(\frac{4}{3} \eta_{\mathrm{s}}(f)+\eta_{\mathrm{v}}(f)\right) \cdot f^{2} .
$$

Durch diese Gleichung werden die in dieser Arbeit gemessenen Absorptionsspektren vollständig beschrieben. Nicht berücksichtigt wurden in (3.2) bzw. in (3.4) Verluste aufgrund von Wärmeleitung im Fluid. Für die hier untersuchten Polymerschmelzen ist ihr Beitrag gegenüber den viskosen Verlusten vernachlässigbar.

Die Ursachen für die frequenz- und substanzabhängigen viskosen Verluste sind im molekularen Aufbau der Flüssigkeiten zu suchen: Molekulare Prozesse führen über Änderungen der mikroskopischen Flüssigkeitstruktur zu Änderungen makroskopischer Größen wie der Scher- und Volumenviskosität. Diese beeinflussen ihrerseits den Absorptionskoeffizenten. Der Zusammenhang zwischen Absorptionsspektren und molekularen Mechanismen wird im Folgenden näher dargestellt.

\subsubsection{Relaxation thermischer Gleichgewichte}

Besonders Flüssigkeitsgemische und Flüssigkeiten mit größeren Molekülen zeigen im Frequenzbereich $10^{3} \mathrm{~Hz}$ bis $10^{9} \mathrm{~Hz}$ so genannte Relaxationsphänomene, die zu einer charakteristischen Erhöhung der Schallabsorption führen. Der Grund dafür ist die Existenz dynamischer Gleichgewichte, die über thermodynamische Größen wie Druck, Temperatur oder Dichte an eine Schallwelle koppeln. Die Schallwelle gibt somit eine leichte Tendenz für die Änderung eines Gleichgewichts vor. Dieser Vorgabe folgt das Gleichgewicht jedoch nicht instantan, sondern mit einer charakteristischen Relaxationszeit. Bestimmt durch das Verhältnis von Relaxationszeit und Periodendauer führt die zeitliche Verzögerung, mit der sich das neue Gleichgewicht einstellt, zu einer Phasenverschiebung zwischen den Druck- bzw. Temperaturschwankungen der Schallwelle und den periodischen Gleichgewichtsänderungen und damit zu einer frequenzabhängigen Absorption von Schallenergie. Molekulare Prozesse, die über geringfügige Störungen ihrer Gleichgewichte im Absorptionsspektrum beobachtet werden können, sind beispielsweise Konformationsänderungen von Molekülen, Komplexbildungen oder Konzentrationsfluktuationen in Flüssigkeiten.

Für die theoretische Beschreibung der Spektren sind je nach Absorptionsmechanismus unterschiedliche Modelle erforderlich. Das zur Auswertung der gemessene Daten verwendete Modell wird im nächsten Abschnitt beschrieben. 


\subsubsection{Relaxationen mit diskreter Relaxationszeit}

Betrachtet wird ein dynamisches Gleichgewicht zwischen zwei diskreten Zuständen, die durch eine Energiebarriere voneinander getrennt sind. Auf äußere Störungen reagiert dieses System, indem es mit einer diskreten Relaxationszeit $\tau_{\mathrm{D}}$ exponentiell in das neue, von außen vorgegebene Gleichgewicht relaxiert. Harmonische Störungen führen zu einer frequenzabhängigen Absorption von Schallenergie, die durch die Debye-Spektralfunktion

$$
(\alpha \lambda)_{\mathrm{D}}=\left(\frac{c_{\mathrm{S}}(\omega)}{c_{\mathrm{s} \infty}}\right)^{2} A_{\mathrm{D}} \frac{\omega \tau_{\mathrm{D}}}{1+\left(\omega \tau_{\mathrm{D}}\right)^{2}}
$$

mit $c_{\mathrm{s} \infty}=c_{\mathrm{s}}(\omega \rightarrow \infty)$ und einer Absorptionsamplitude $A_{\mathrm{D}}$ gegeben ist [25, 26]. Existieren in einer Flüssigkeit $N$ solche Gleichgewichte mit unterschiedlichen Relaxationszeiten $\tau_{\mathrm{D}_{i}}$, so wird die Absorption entlang einer Wellenlänge durch

$$
(\alpha \lambda)_{\mathrm{D}}=\left(\frac{c_{\mathrm{s}}(\omega)}{c_{\mathrm{s} \infty}}\right)^{2} \sum_{i=1}^{N} A_{\mathrm{D}_{i}} \frac{\omega \tau_{\mathrm{D}_{i}}}{1+\left(\omega \tau_{\mathrm{D}_{i}}\right)^{2}}
$$

beschrieben. Mit steigender Frequenz können Molekülumordnungen in der Flüssigkeit dem äußeren Zwang immer weniger folgen, wodurch es zu einer Abnahme der Kompressibilität und einer Dispersion der Schallgeschwindigkeit kommt, die für eine Summe von Einzelzeitrelaxationen die Form

$$
\left(\frac{c_{\mathrm{S} \infty}}{c_{\mathrm{S}}(\omega)}\right)^{2}=1+\frac{1}{\pi} \sum_{i=1}^{N} A_{\mathrm{D}_{i}} \frac{1}{1+\left(\omega \tau_{\mathrm{D}_{i}}\right)^{2}}
$$

besitzt. Im betrachteten Frequenzbereich ist die beobachtete Dispersion für viele Flüssigkeiten sehr klein, so dass in guter Näherung $c_{\mathrm{s} \infty} / c_{\mathrm{s}}(\omega) \approx 1$ gesetzt werden kann. Bei den untersuchten polymeren Substanzen gewinnt sie aber mit zunehmender Kettenlänge bzw. Schallabsorption an Bedeutung (vergl. Abschnitt 4.2.2).

\subsubsection{Hintergrundsanteil}

Häufig werden Ultraschallspektren frequenznormiert, d.h. in der Form $\alpha / f^{2}$ über $f$ dargestellt. Aus Gleichung (3.5) erhält man in dieser Darstellung für die DebyeSpektralfunktion

$$
\left(\frac{\alpha}{f^{2}}\right)_{\mathrm{D}}=2 \pi \cdot\left(\frac{c_{\mathrm{s}}(\omega)}{c_{\mathrm{s} \infty}^{2}}\right) A_{\mathrm{D}} \frac{\tau_{D}}{1+\left(\omega \tau_{\mathrm{D}}\right)^{2}}
$$

Für $\omega \rightarrow 0$ ist der Quotient $\alpha / f^{2}$ konstant, für $\omega \rightarrow \infty$ geht er gegen 0. Diskrete Gleichgewichte, die weit oberhalb des betrachteten Frequenzbereichs relaxieren, tragen in dieser Darstellung zum gemessenen Spektralbereich also nur mit einem konstanten Anteil bei. Da über diese Prozesse keine Aussagen gemacht werden 
können, fasst man ihre tieffrequenten Beiträge zu einem frequenzunabhängigen Hintergrundsanteil, dem so genannten $B$-Wert, zusammen und schreibt:

$$
\left(\frac{\alpha}{f^{2}}\right)=2 \pi \cdot\left(\frac{c_{\mathrm{s}}(\omega)}{c_{\mathrm{s} \infty}^{2}}\right) \sum_{i=1}^{K} A_{\mathrm{D}_{i}} \frac{\tau_{\mathrm{D}_{i}}}{1+\left(\omega \tau_{\mathrm{D}_{i}}\right)^{2}}+\frac{B}{c_{\mathrm{s} \infty}}
$$

oder in der $(\alpha \lambda)$-Darstellung

$$
(\alpha \lambda)=\left(\frac{c_{\mathrm{s}}(\omega)}{c_{\mathrm{s} \infty}}\right)^{2} \sum_{i=1}^{K} A_{\mathrm{D}_{i}} \frac{\omega \tau_{\mathrm{D}_{i}}}{1+\left(\omega \tau_{\mathrm{D}_{i}}\right)^{2}}+B f,
$$

wobei $K<N$ die Anzahl der im Messbereich relaxierenden molekularen Prozesse ist.

Durch die Funktionen (3.9) bzw. (3.10) werden alle in dieser Arbeit vorgestellten Ultraschallabsorptionsspektren beschrieben.

\subsection{Messverfahren}

Die mit Hilfe der Ultraschallabsorptionsspektroskopie beobachteten Relaxationsprozesse erstrecken sich über sehr große Frequenzbereiche. Bereits eine Einzelzeitrelaxation, wie durch Gleichung (3.5) beschrieben, besitzt eine Halbwertsbreite von etwa einer Frequenzdekade. Um Fehler bei der Anpassung der Spektralfunktionen und damit bei der Interpretation der zugrunde liegenden molekularen Vorgänge zu vermeiden, muss der Absorptionskoeffizient daher über einen ausreichend breiten Spektralbereich gemessen werden. Mit den in dieser Arbeit verwendeten Apparaturen wurde ein Frequenzbereich von $200 \mathrm{kHz}$ bis $2 \mathrm{GHz}$ erfasst. Der große Wertebereich, den der Absorptionskoeffizient innerhalb dieses Intervalls überstreicht, erfordert den Einsatz verschiedener Messverfahren und apparativer Aufbauten:

Oberhalb von $5 \mathrm{MHz}$ wird das Schwingungspulstransmissionsverfahren genutzt, bei dem der Absorptionskoeffizient direkt aus dem Abfall der Schalldruckamplitude längs einer veränderlichen Messstrecke bestimmt wird. Zu tieferen Frequenzen hin wird mit abnehmender Dämpfung die Messstrecke, die für ein aussagekräftiges Ergebnis notwendig wäre, unpraktikabel groß (vergl. Gl.(3.9)). Man geht daher zum Resonatorverfahren über. Hier wird die effektive Weglänge, die die Schallwelle durchläuft, durch Mehrfachreflexion erhöht. Bei ausgezeichneten Frequenzen bilden sich in der Messzelle Resonanzen aus, aus deren Halbwertsbreite der Absorptionskoeffizient bestimmt werden kann. 


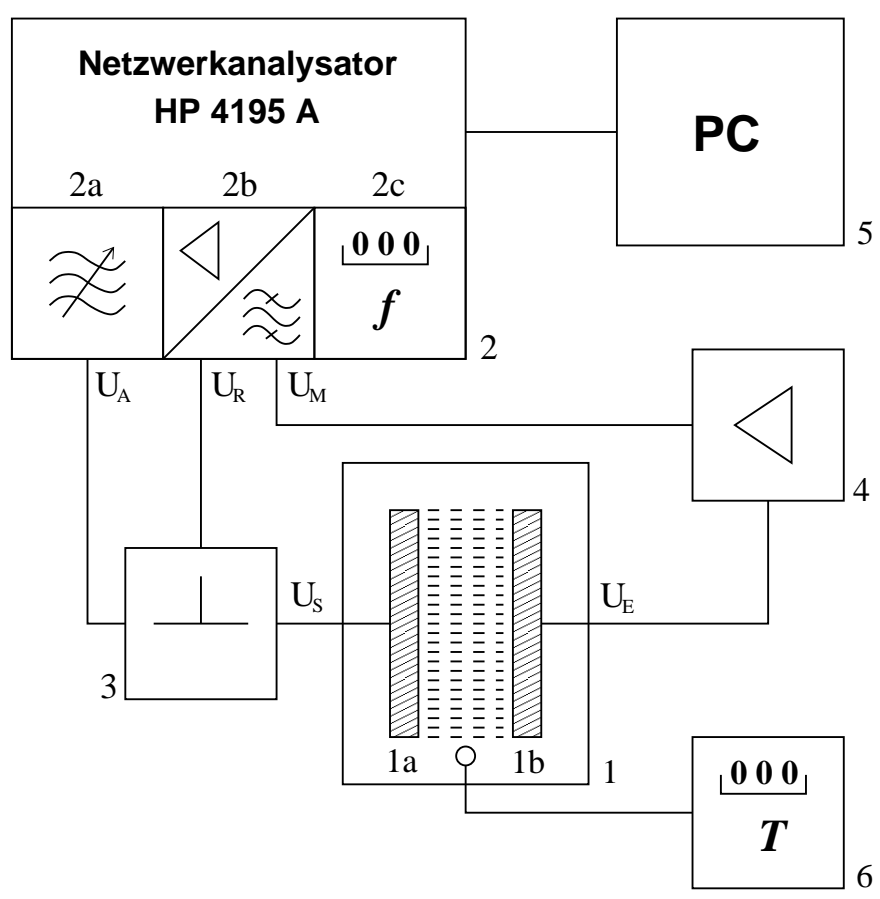

Abbildung 3.1: Blockschaltbild des Resonatormessplatzes nach [27]: (1) Messzelle mit (1a) Sendeund (1b) Empfangswandler, (2) Netzwerkanalysator mit (2a) Signalausgang und den Eingängen für (2b) Referenz- und (2c) Empfangssignal, (3) Leistungsteiler, (4) Vorverstärker, (5) Prozessrechner, (6) Digitalthermometer.

\subsubsection{Resonatorverfahren}

\subsubsection{Messprinzip}

Apparativer Aufbau Abb. 3.1 zeigt ein Blockschaltbild der Apparatur. Den Resonator bildet eine zylinderförmige, vollständig mit der zu untersuchenden Flüssigkeit gefüllte Messzelle (1), deren Stirnseiten von zwei Ultraschallwandlern (1a, 1b) verschlossen werden. Sie bestehen aus piezoelektrischen Quarzscheiben, deren Flächen zum Anschluss an die elektronische Apparatur mit Chrom und anschließend mit Gold bedampft sind. Zur Messung sendet der Signalgenerator (2a) eines Netzwerkanalysators (NWA, HP8751A) (2) kontinuierlich eine sinusförmige Wechselspannung $U_{\mathrm{A}}$ aus. Ein Leistungsteiler (3) spaltet dies Signal in zwei Anteile auf. Der eine Teil, die Referenzspannung $U_{\mathrm{R}}$, wird direkt an den Vergleichseingang (2b) des NWA zurückgeführt. Durch den zweiten Anteil, dem zu $U_{\mathrm{R}}$ proportionalen Messsignal $U_{\mathrm{S}}$, wird der Sendewandler (1a) zu Dickenschwingungen gleicher Frequenz angeregt und strahlt eine Schallwelle in die Flüssigkeit ab, deren Schalldruck proportional zur Spannung $U_{\mathrm{S}}$ ist. Am Empfangs- bzw. Sendewandler wird die Schallwelle reflektiert und durchläuft auf diese Weise mehrfach die Flüssigkeit. Bei bestimmten Frequenzen bildet die Messzelle ein Resonanzsystem. Mit Hilfe des Empfangswandlers (1b) wird das entstehende Stehwellenfeld detektiert. An ihm lässt sich eine Spannung $U_{\mathrm{E}}$ abgreifen, die dem am Wandler vorherrschenden Schalldruck proportional ist. Diese wird amplitudenabhängig, aber linear, verstärkt 
(4) und dem Messeingang (2c) des NWA als $U_{\mathrm{M}}$ zugeführt. Aus dem Spannungsverhältnis $U_{\mathrm{M}} / U_{\mathrm{R}} \sim U_{\mathrm{E}} / U_{\mathrm{S}}$ wird die komplexe Übertragungsfunktion des Systems berechnet, mit deren Hilfe, wie in den nächsten Abschnitten erläutert, der Absorptionskoeffizient ermittelt werden kann. Ein Rechner (5) übernimmt die Steuerung des NWA und des Verstärkers sowie die Verarbeitung der vom Analysator bereitgestellten Messdaten. Während der Messung wird die Temperatur über ein Digitalthermometer (6) kontrolliert, dessen Fühler möglichst dicht am inneren Zellmantel positioniert wird. Zur Stabilisierung der Temperatur wird zum einen der Zellmantel selbst thermostatiert, zum anderen befindet sich der Resonator in einer thermostatierten Kammer.

\section{Bestimmung des Absorptionskoeffizienten aus der Übertragungsfunktion}

Betrachtet wird ein idealisierter zylindrischer Resonator, bestehend aus zwei planparallelen, im Abstand $L$ angeordneten dünnen Ultraschallwandlern, zwischen denen sich die Messflüssigkeit befindet. Der Sendewandler strahle eine ebene Schallwelle mit Frequenz $f$ und einer Wellenlänge $\lambda$ aus, die klein gegenüber dem Radius der Wandlerplatten sei. Die Welle werde beim Durchlaufen der Flüssigkeitsschicht mit einem Absorptionskoeffizienten $\alpha$ gedämpft und an den als schallhart angenommenen Grenzschichten Flüssigkeit-Wandler vollständig reflektiert. Dann erhält man durch Aufsummieren der momentanen Schalldrücke aller am Empfangswandler ein- und auslaufendenden Wellen die Spannung $U_{\mathrm{E}}$

$$
U_{\mathrm{E}} \sim e^{-\gamma L}+e^{-3 \gamma L}+\ldots
$$

und daraus die Übertragungsfunktion [28]

$$
T(f)=\frac{U_{\mathrm{E}}}{U_{\mathrm{S}}}=\hat{T} \frac{1}{\sinh (\gamma L)}
$$

mit der Ausbreitungskonstante $\gamma=\alpha+i \frac{2 \pi}{\lambda}$ und einer Amplitude $\hat{T}$, die ein Maß für den Transfer von elektrischer in akustische Leistung an den piezoelektrischen Wandlern darstellt. Die Übertragungsfunktion zeigt Maxima an äquidistanten Stellen, den Resonanzfrequenzen

$$
f_{n}=n \frac{c_{\mathrm{s}}}{2 L} \quad \text { für } n \in \mathbb{N} .
$$

Diese Resonanzen werden im Folgenden als Hauptmoden bezeichnet. Für hinreichend kleine Dämpfungen $\alpha L \ll 1$ lässt sich der Verlauf der Übertragungsfunktion in der Nähe einer Resonanzfrequenz $f_{n}$ durch Taylorentwicklung bestimmen. Aus der Leistungshalbwertsbreite $\Delta f_{n}$ der $n$-ten Resonanz erhält man dann für den Absorptionskoeffizienten

$$
\alpha=\frac{\pi}{c_{\mathrm{s}}} \Delta f_{n}
$$

Die Schallgeschwindigkeit kann bei bekannter Resonatorzelllänge aus dem Abstand der Resonanzen nach Gleichung (3.12) ermittelt werden. 


\subsubsection{Berücksichtigung apparativer Verluste - Referenzmessung}

Im letzten Abschnitt wurde zunächst davon ausgegangen, dass die am Empfangswandler registrierte Abnahme der Schalldruckamplitude allein auf Relaxationsprozesse in der Messflüssigkeit zurückzuführen ist. Unter realen Bedingungen treten jedoch zusätzlich zur Flüssigkeitsabsorption noch weitere Verlustmechanismen auf, die im Folgenden beschrieben werden.

Verluste durch Beugung Aufgrund der endlichen Wandlerdurchmesser kommt es zu Beugungseffekten, die infolge des nicht verschwindenden Wellenwiderstands der Zellberandung mit einer Dissipation von Schallenergie verbunden sind. Da die Verluste stark von der Schallintensität im Randbereich des Resonators abhängen, werden zum Teil konkave anstelle planer Ultraschallwandler eingesetzt, die eine Einschnürung des Wellenfeldes zur Mittelachse hin bewirken und die Beugungsverluste in tiefere Frequenzbereiche verschieben [22].

Im Fall eines plan-planen Resonators ist der Beitrag $\left(\Delta f_{n}\right)_{\mathrm{B}}$ der Beugung zur gemessenen Halbwertsbreite in [29] berechnet worden. Für einen Resonator mit Wandlerradius $R_{\mathrm{q}}$, Zelllänge $L$ und einer Zellberandung mit Schallgeschwindigkeit $c_{\mathrm{b}}$ und Dichte $\rho_{\mathrm{b}}$, der mit einer Flüssigkeit der Dichte $\rho$ und Schallgeschwindigkeit $c_{\mathrm{s}}$ befüllt ist, gilt demnach für $10 \leq 2 \pi \hat{\beta} R_{\mathrm{q}} / L \leq 500$

$$
\frac{\left(\Delta f_{n}\right)_{\mathrm{B}}}{f_{n}}=\frac{0.147}{\hat{\beta}}\left(\frac{c_{\mathrm{s}}}{R_{\mathrm{q}} f_{n}}\right)^{3}
$$

mit der spezifischen Eingangsimpedanz $\hat{\beta}=\left(\rho c_{\mathrm{s}}\right) /\left(\rho_{\mathrm{b}} c_{\mathrm{b}}\right)$. Für plan-konkave bzw. bikonkave Resonatoren ist keine vergleichbare Beschreibung bekannt.

Verluste im Bereich von Quarzdicken- und Scherresonanzen Bis hierher wurde die Grenzschicht zwischen Flüssigkeit und Wandler als schallhart angenommen. In der Realität ist die Wandlerimpedanz jedoch nicht unendlich groß, so dass das Wellenfeld in den Wandlerquarz eindringen kann. Die Ursache dafür ist, dass der Wandler aufgrund seiner endlichen Dicke ein mechanisches Resonanzsystem mit äquidistanten Resonanzfrequenzen $f_{\mathrm{q} n}$ bildet, von denen ungeradzahlige Vielfache der Grundresonanz $f_{\mathrm{q} 1}$ elektrisch angeregt werden können. Mit Hilfe der Leitungstheorie lässt sich die Eindringtiefe berechnen. Sie stellt sich als frequenzabhängig heraus [30]: Nur für Zellresonanzen, die genau zwischen zwei Quarzdickenresonanzen liegen, wird der schallweiche Quarz-Luft-Übergang auf der Wandlerrückseite in einen schallharten Übergang zwischen Quarz und Flüssigkeit transformiert, so dass die Zelllänge dem geometrischen Wandlerabstand entspricht. Für $f \rightarrow f_{\mathrm{q} n}$ nimmt der Betrag des Reflexionsfaktors ab und die Schalldruckbäuche verlagern sich in den Wandler. Diese Änderung der akustischen Wandlerimpedanz im Bereich der Quarzdickenresonanzen bewirkt eine verstärkte Abstrahlung von Schallenergie am Quarz-Luft-Übergang auf der Wandlerrückseite. 
Auch bei X-Schnitt Quarzen können aufgrund der elektro-mechanischen Ankopplung im Kristall Scherresonanzen angeregt werden. Ihre Grundmode liegt bei etwa 3/5 der Dickengrundresonanz. Zu höheren Frequenzen folgen die entsprechenden Harmonischen. Ihre Anregung ist mit einer verstärkten Schallabstrahlung in die seitlichen Wandlerhalterungen verbunden.

Neben den beschriebenen sind noch weitere Verlustmechanismen denkbar. Ihnen allen ist gemein, dass ihr Beitrag zur gemessenen Halbwertsbreite von vielen verschiedenen Bedingungen wie z.B. der Messfrequenz, der Messsubstanz oder dem aktuellen Zustand des Resonators abhängt, und sich in der Praxis bestenfalls abschätzen lässt. Eine sichere theoretische Vorhersage ist nicht möglich. Um die apparativen Verluste dennoch klar von der Schallabsorption in der Messsubstanz zu trennen, wird eine Referenzmessung an einer Flüssigkeit vorgenommen, deren Absorptionskoeffizient bekannt ist. Unter der Annahme, dass sich die durch Flüssigkeitsdämpfung bedingten und die apparativen Verluste additiv verhalten, lässt sich dann der Flüssigkeitsbeitrag $\left(\Delta f_{n}\right)_{\mathrm{fl}}$ zur Halbwertsbreite auf folgende Weise bestimmen:

$$
\left(\Delta f_{n}\right)_{\mathrm{fl}}=\alpha \frac{c_{\mathrm{s}}}{\pi}=\left(\Delta f_{n}\right)_{\mathrm{mess}}-\left(\Delta f_{n}\right)_{\mathrm{app}} .
$$

Der Anteil $\left(\Delta f_{n}\right)_{\text {app }}$ ergibt sich aus der Referenzmessung durch

$$
\left(\Delta f_{n}\right)_{\mathrm{app}}=\left(\Delta f_{n}\right)_{\mathrm{refmess}}-\left(\Delta f_{n}\right)_{\mathrm{ref}} .
$$

Dabei steht $\left(\Delta f_{n}\right)_{\text {mess }}$ für die Gesamthalbwertsbreite bei der Substanzmessung, $\left(\Delta f_{n}\right)_{\text {app }}$ für den apparativen Anteil, $\left(\Delta f_{n}\right)_{\text {refmess }}$ für die Gesamthalbwertsbreite der Referenzmessung und $\left(\Delta f_{n}\right)_{\text {ref }}$ für die Halbwertsbreite aufgrund der bekannten Flüssigkeitsabsorption der Referenz. Voraussetzung ist allerdings, dass die Referenzflüssigkeit Eigenschaften besitzt, die zu den gleichen apparativen Verlusten führen wie die Messsubstanz. Um möglichst gleiche Beugungsverluste zu erhalten, sollten nach Gleichung (3.14) Schallgeschwindigkeit (sie geht hier quadratisch ein) und Dichte übereinstimmen. Dies ist auch sinnvoll, um das Abstrahlungsverhalten der Wandler nicht durch einen unterschiedlichen Reflexionsfaktor zu verändern.

Verwendete Referenzsubstanzen Für die Auswertung der OligoethylenglykolDaten wurden zunächst Harnstoff-Wasser-Lösungen verwendet. Dabei handelt es sich um eine Standardreferenz, deren konzentrationsabhängige Verläufe von Schallgeschwindigkeit, Dichte und Absorptionskoeffizienten sehr genau bekannt sind [31]. Im betrachteten Frequenzbereich zeigt Harnstoff keine Relaxationsstufen. Die Dichten der Oligoethylenglykole und der Harnstofflösungen stimmen ausreichend gut überein (vergl. Tab. 4.1), die Schallgeschwindigkeit kann über die Harnstoffkonzentration mit großer Genauigkeit festgelegt werden. Der Absorptionskoeffizient der Lösungen, der immer im Bereich $\alpha \approx 19 \cdot 10^{-15}\left(\mathrm{~s}^{2} / \mathrm{m}\right) \cdot f^{2}$ liegt, unterschreitet jedoch die Absorptionskoeffizienten der verwendeten Oligoethylenglykole um einen Faktor 2 bis 40 . 
Empirisch zeigt sich, dass neben der Dichte und der Schallgeschwindigkeit auch die Flüssigkeitsdämpfung die Beugungsverluste beeinflusst. Bei großen Unterschieden zwischen den Absorptionskoeffizienten von Referenz und Messsubstanz wie im vorliegenden Fall treffen die Gleichungen (3.15) und (3.16) also nicht mehr notwendig zu. Um systematische Fehler bei der Interpretation der Spektren zu vermeiden, wurde deshalb in einer zweiten Auswertungsreihe ein Teil der Polymerspektren gegen eine andere Referenz ausgewertet. Soweit geeignete Messdaten vorhanden waren, wurde dabei immer das nächst längere Polymer der gleichen Sorte als Referenz gewählt. Dadurch war zwar der Fehler in der Schallgeschwindigkeit etwas größer $(<3 \%)$, der Unterschied in der Dämpfung aber deutlich kleiner (Tab. 4.1). In Abb. 3.2 ist zu erkennen, wie sich die unterschiedlichen Referenzeigenschaften insbesondere im tieffrequenten Bereich, der von den Beugungsverlusten dominiert wird, auswirken. Eine ausführlichere Diskussion der Auswertungsergebnisse findet sich in Abschnitt 4.1.

Für die Messung der Polypropylenglykol- und der Oligoethylenglykoldimethylether-Schmelzen wurden Ethanol-Wasser-Lösungen als Referenz verwendet.

Korrektur nach Kononenko Nach [32] beeinflusst die Flüssigkeitsdämpfung die apparativen Verluste im Resonator noch durch einen weiteren Effekt. Verantwortlich ist der Unterschied zwischen der Schallabsorption im Wandler und der in der Flüssigkeit. Laut Kononenko ist der Resonator dadurch als mehrstufiges System mit unterschiedlichen Absorptionseigenschaften aufzufassen. Gemessen wird jedoch die Übertragungsfunktion des gesamten Systems. Vor allem im Bereich der Quarzresonanzen, in denen das Schallfeld deutlich in den Wandler eindringt, wird daher die Absorption der Flüssigkeit, je nachdem ob sie höher oder niedriger ist als die der Wandler, zu klein bzw. zu groß bestimmt. Um diesen Effekt zu berücksichtigen, werden die gemessenen Halbwertsbreiten $\left(\Delta f_{n}\right)_{\text {mess }}$ und $\left(\Delta f_{n}\right)_{\text {refmess }}$ bei Substanzund Referenzmessung vor ihrer Korrektur nach Gl. (3.15)/ (3.16) entsprechend der in [32] vorgeschlagenen Gleichung korrigiert:

$$
\left(\Delta f_{n}\right)_{\mathrm{mess}}^{\mathrm{kor}}=\left(\Delta f_{n}\right)_{\mathrm{mess}}\left(1+z \mu\left(1-Q_{\mathrm{q}}^{-1} \frac{f_{n}}{\left(\Delta f_{n}\right)_{\mathrm{mess}}}\right)\right)
$$

wobei

$$
z=2 \frac{c_{\mathrm{s}} D}{c_{\mathrm{q}} L}, \quad \mu^{-1}=\zeta \cos ^{2}\left(\pi f_{n} / f_{\mathrm{q} 1}\right)+\zeta^{-1} \sin ^{2}\left(\pi f_{n} / f_{\mathrm{q} 1}\right), \quad \zeta=\frac{\rho c_{\mathrm{s}}}{\rho_{\mathrm{q}} c_{\mathrm{q}}},
$$

mit einer Wandlerdicke $D$, Wandlerschallgeschwindigkeit $c_{\mathrm{q}} \approx 5700 \mathrm{~m} / \mathrm{s}$, Wandlerdichte $\rho_{\mathrm{q}}$ und einer Wandlergüte $Q_{\mathrm{q}}$, die das Verhältnis von gespeicherter zu dissipierter Energie im Quarz beschreibt. Aufgrund von Messungen an Substanzen bekannter Flüssigkeitsdämpfung [22] wird bei der Korrektur $Q_{\mathrm{q}}=15000$ gesetzt. Der genaue Wert von $Q_{\mathrm{q}}$ ist nicht ausschlaggebend, da sich der Effekt vor allem im Bereich der Quarzdickenresonanzen auswirkt, der jedoch bei der Auswertung der Daten ohnehin nicht berücksichtigt wird. Im messtechnisch günstigen Bereich 

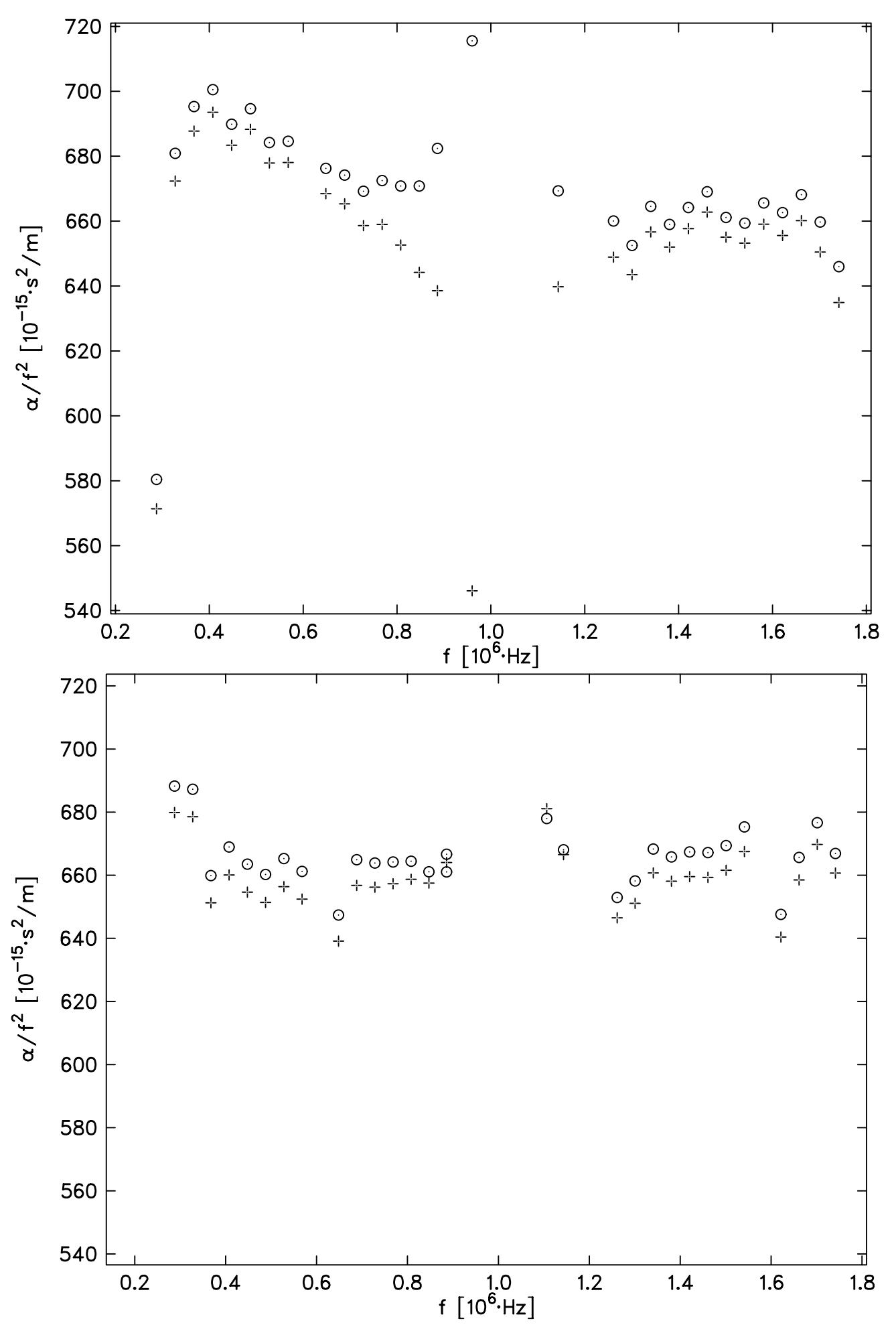

Abbildung 3.2: Kononenko-korrigierte $(\odot)$ und -unkorrigierte $(+)$ Messdaten des $1 \mathrm{MHz}-$ Resonators für PEG 300 bei $25^{\circ} \mathrm{C}$. Oben: Referenz Harnstoff-Wasser, unten: Referenz PEG 400 (II). 
außerhalb der Umgebung $(1 \pm 0.2) \cdot f_{\mathrm{q} n}$ weichen die Kononenko-korrigierten Absorptionswerte bei den in dieser Arbeit betrachteten Substanzen um maximal 2.5\% von den unkorrigierten ab. Wie an Gleichung (3.17) zu sehen ist, gewinnt die Korrektur an Bedeutung, wenn sich Referenz- und Messsubstanz in ihren Absorptionskoeffizienten deutlich unterscheiden. Das zeigt sich auch bei den Resonatordaten der Oligoethylenglykole, die gegen zwei unterschiedliche Referenzen ausgewertet wurden (siehe Abb. 3.2).

\subsubsection{Bestimmung von Zelllänge und Schallgeschwindigkeit - Wassermessung}

Die Möglichkeit, aus dem Hauptmodenabstand nach Gleichung (3.12) die Schallgeschwindigkeit zu bestimmen, ist beim plan-konkaven oder bikonkaven Resonator dadurch erschwert, dass bei konkaver Wandlergeometrie keine eindeutige Zelllänge angegeben werden kann. Vor allem aber führt das in Abschnitt 3.2.1.2 beschriebene Eindringen des Schallfeldes in den Ultraschallwandler zu einer merklichen frequenzabhängigen Änderung der Zelllänge. Damit verbunden ist eine Verschiebung der Resonanzfrequenzen, die nach Berechnungen von Labhardt [30] nicht mehr äquidistant liegen. Unter Vernachlässigung der Flüssigkeitsdämpfung, der rückwärtigen bzw. seitlichen Schallabstrahlung und der endlichen Wandlerradien ergibt sich für den plan-planen Resonator:

$$
f_{n}-f_{n-1}=\frac{c_{\mathrm{s}}}{2 \pi L} \arccos \left(\frac{\left(g_{n}^{2}-1\right)\left(1-g_{n-1}^{2}\right)-4 g_{n} g_{n-1}}{\left(1+g_{n}^{2}\right)\left(1+g_{n-1}^{2}\right)}\right)
$$

mit

$$
g_{n}=\frac{\rho c_{\mathrm{s}}}{\rho_{\mathrm{q}} c_{\mathrm{q}} \tan \left(\frac{\pi f_{n}}{f_{\mathrm{q} 1}}\right)}
$$

Eine Bestimmung der Schallgeschwindigkeit ist dennoch auf folgende Weise möglich: Mit einer Wassermessung werden die Resonanzfrequenzen des Resonators ermittelt und eine effektive Zelllänge $L_{\text {eff }}$ bestimmt. Dazu wird über alle Einzelwerte von $L$ gemittelt, die sich aus den Frequenzabständen benachbarter Hauptmoden ergeben. Anschließend werden die Resonanzfrequenzen für die zu untersuchende Substanz ermittelt. Aus ihrer Lage kann mit Hilfe der effektiven Zelllänge die Schallgeschwindigkeit $c_{\mathrm{s}}$ iterativ aus (3.18) errechnet werden. Es hat sich gezeigt, dass Gleichung (3.18) auch für die verwendeten plan-konkaven und bikonkaven Resonatoren sinnvolle Schallgeschwindigkeiten liefert.

\subsubsection{Berücksichtigung von Nebenmoden und Übersprechen}

Nebenmoden Aufgrund der zylindrischen Resonatorgeometrie und der zum Teil gekrümmten Wandleroberflächen treten neben den Hauptresonanzen weitere Moden mit zusätzlichen radialen Knotenebenen auf, die sich von der hochfrequenten Seite her an die Hauptmoden anschließen. Sie werden als Nebenmoden bezeichnet. Im Fall eines plan-planen Resonators liegt die $j$-te Nebenmode zur $n$-ten 
Hauptmode bei der Frequenz [30]

$$
f_{n, j}=f_{n}+\frac{c_{\mathrm{s}}^{2}}{8 R_{\mathrm{q}}^{2}} \frac{(j-1 / 4)^{2}-(3 / 4)^{2}}{f_{n}} \quad \text { mit } n, j \in \mathbb{N} .
$$

Für den plan-konkaven Resonator mit Krümmungsradius $R_{\mathrm{K}}$ findet man für $\lambda \ll$ $R_{\mathrm{q}}[33]$

$$
f_{n, j}=f_{n}+j \cdot \frac{1}{\pi} \arccos \left(\sqrt{1-L / R_{\mathrm{K}}}\right) \cdot \frac{c_{\mathrm{s}}}{2 L},
$$

für den bikonkaven Fall erhält man unter diesen Bedingungen

$$
f_{n, j}=f_{n}+j \cdot \frac{1}{\pi} \arccos \left(1-L / R_{\mathrm{K}}\right) \cdot \frac{c_{\mathrm{s}}}{2 L} .
$$

Zu tiefen Frequenzen hin beobachtet man experimentell einen Übergang in das Verhalten eines plan-planen Resonators [22, 34], was dadurch zu erklären ist, dass bei zunehmender Wellenlänge die Krümmung immer unbedeutender wird.

Mit zunehmender Flüssigkeitsabsorption nimmt bei höheren Frequenzen auch die Breite der Hauptresonanz und ihrer Nebenmoden zu, wodurch die interessierende Übertragungsfunktion der Hauptmode erheblich gestört werden kann. Der Einfluss der Nebenmoden wird bei der Auswertung berücksichtigt, indem man an die Messdaten eine Summe komplexwertiger Funktionen nach Gleichung (3.11) anpasst. Mit Hilfe der Anpassungsparameter (Abschnitt 3.2.1.5) kann dann die Übertragungsfunktion der Hauptmode aus den Messergebnissen herausgerechnet werden.

Elektrisches Übersprechen Eine weitere mögliche Störung der Übertragungsfunktion ist das elektrische Übersprechen zwischen Sende- und Empfangswandler. Vor allem dort, wo die Amplituden der akustischen Zellresonanzen klein sind und besonders verstärkt werden müssen, macht es sich durch eine erhöhte Empfängerspannung bemerkbar. Da sich das Übersprechen über das Frequenzintervall einer Zellresonanz kaum ändert, kann es durch eine komplexe additive Konstante $A_{\mathrm{u}} e^{i \phi_{\mathrm{u}}}$ mit Amplitude $A_{\mathrm{u}}$ und Phase $\phi_{\mathrm{u}}$ bei der Anpassung der Übertragungsfunktion miteinbezogen werden (s. Abschnitt 3.2.1.5)

\subsubsection{Anpassrechnung}

Unter Berücksichtigung der Nebenmoden und des elektrischen Übersprechens wird an die gemessene Übertragungsfunktion die folgende Funktion angepasst:

$$
T(f)=\sum_{j=1}^{N}\left(\frac{\hat{T}_{j}}{\sinh \left(\gamma_{j}(f) \cdot L\right)}\right)+A_{\mathrm{u}} e^{i \phi_{\mathrm{u}}}
$$

Dabei bezeichnet: 

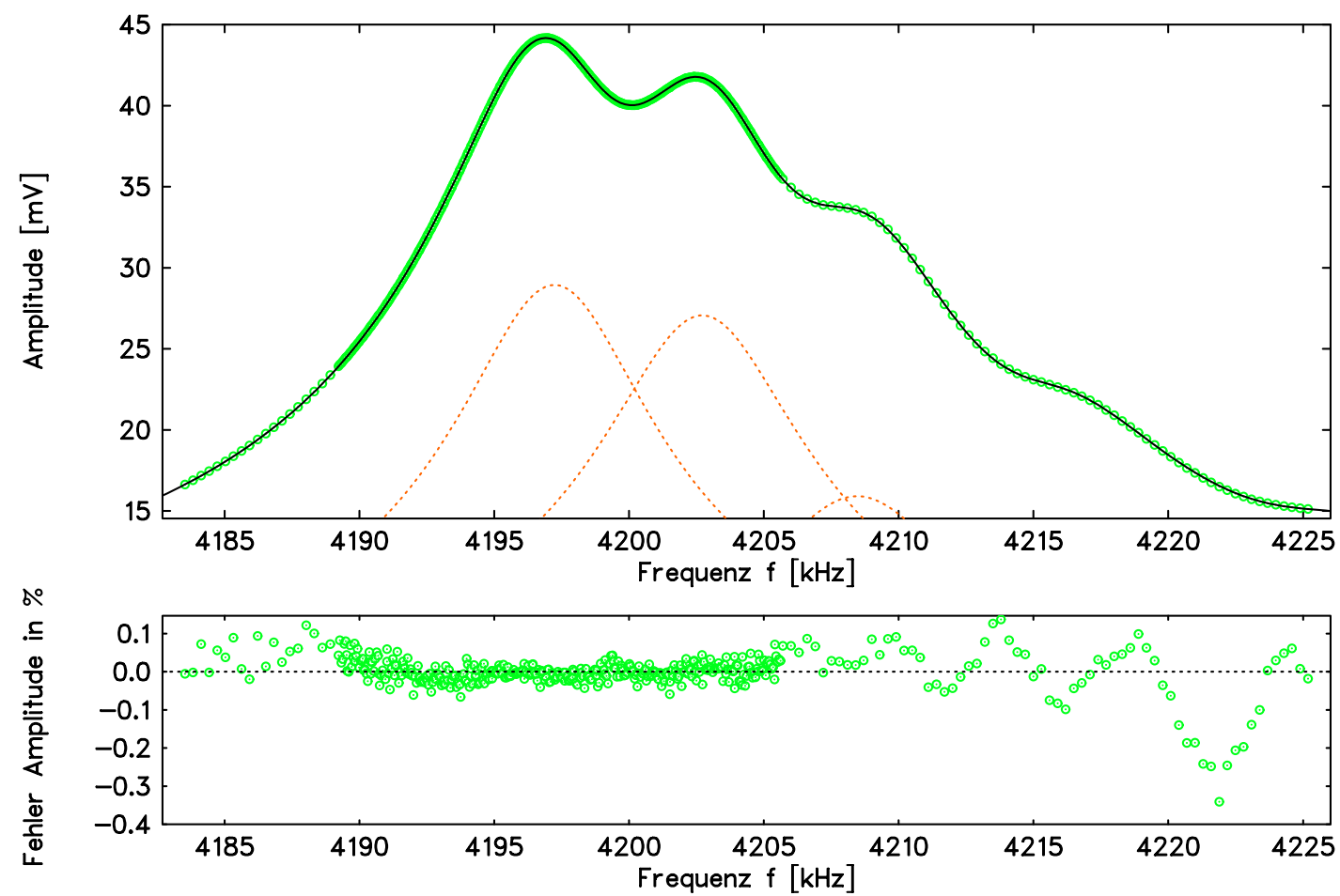

Abbildung 3.3: Resonanz bei einer Frequenz um $4200 \mathrm{kHz}$, angepasst wurden die Hauptmode und vier Nebenmoden, Messung an PEG 400 (II) bei $25^{\circ}$ C. Symbole (o) : gemessene Amplitude; durchgezogene Kurve: Betrag der nach Gl. (3.22) angepassten Funktion $T(f)$; gestrichelte Kurven: einzeln betrachtete Betragsfunktionen der Hauptmode und der ersten beiden Nebenmoden. Die untere Abbildung zeigt die Abweichung der Messdaten von der angepassten Funktion.

$$
\begin{aligned}
& \gamma_{j}(f)=\alpha_{j}+i \frac{2 \pi}{c_{\mathrm{s}}}\left(f-f_{n, j}\right) \quad: \quad \text { Ausbreitungskonstante der } j \text {-ten Mode } \\
& \text { ( } j=1 \text { : Hauptmode, } j=2, \ldots, N \text { : Nebenmoden }) \\
& \hat{T}_{j}: \text { Amplitude der } j \text {-ten Mode } \\
& \alpha_{j}=\frac{\pi}{c_{\mathrm{s}}} \Delta f_{n, j} \quad: \quad \text { Dämpfungswert der } j \text {-ten Mode } \\
& f_{n, j}, \Delta f_{n, j} \text { : Resonanzfrequenz und Halbwertsbreite der } j \text {-ten } \\
& \text { Nebenresonanz zur n-ten Hauptmode } \\
& A_{\mathrm{u}} \quad \text { : Amplitude des elektrischen Übersprechens } \\
& \phi_{\mathrm{u}} \text { : Phase des elektrischen Übersprechens }
\end{aligned}
$$

Je Mode gibt es also drei freie Parameter: $f_{n, j}, T_{j}$ und $\alpha_{j}$ (bzw. die Halbwertsbreite der j-ten Mode $\Delta f_{n, j}$ ). Hinzu kommen Amplituden- und Phasenparameter des Übersprechens. Maximal wurden 4 Nebenmoden angepasst. Höhere Moden, die bei starker Flüssigkeitsabsorption zur Übertragungsfunktion beitragen, konnten durch die vierte Nebenmode ausreichend gut mitbeschrieben werden. Eine Erweiterung 
auf mehr als vier Nebenmoden erscheint nicht sinnvoll: Bei den vorliegenden hohen Dämpfungswerten ist der Überlapp der Moden so groß, dass sie kaum noch spektral zu trennen sind. Weitere freie Parameter würden hier nicht zu einer signifikanten Verbesserung der Ergebnisse führen, die Anpassung aber deutlich erschweren. Ausschlaggebend für eine gelungene Anpassung ist eine möglichst statistische Abweichung der Messpunkte von der angepassten Übertragungsfunktion, eine möglichst geringe Modenanzahl, deren Zunahme zu höheren Messfrequenzen hin konsistent ist, und ein sinnvoller Parameterverlauf sowohl zwischen den Nebenmoden einer Hauptresonanz als auch zwischen benachbarten Hauptmoden.

\subsubsection{Die verwendeten Resonatormesszellen}

In der nachfolgenden Tabelle sind die wichtigsten Eigenschaften der verwendeten Resonatoren dargestellt. Außerdem ist der relative Fehler im Absorptionskoeffizienten, basierend auf eigenen Erfahrungen und Erfahrungen anderer, angegeben. Einige wesentliche Fehlerquellen werden im nächsten Abschnitt genauer diskutiert. Da die Fehler stark von den Eigenschaften der Messsubstanzen und von den Messfrequenzen abhängen, sind die angegebenen Werte nur als Richtgrößen anzusehen. Grundsätzlich gilt aber, dass mit kleiner werdender Flüssigkeitsabsorption und kleiner werdenden Frequenzen der Fehler deutlich zunimmt.

\begin{tabular}{lcccccccc}
$\begin{array}{l}\text { Resonator/ } \\
\text { Geometrie }\end{array}$ & $\begin{array}{c}f_{\mathrm{q}} \\
{[\mathrm{MHz}]}\end{array}$ & $\begin{array}{c}R_{\mathrm{q}} \\
{[\mathrm{mm}]}\end{array}$ & $\begin{array}{c}R_{\mathrm{K}} \\
{[\mathrm{m}]}\end{array}$ & $\begin{array}{c}R \\
{[\mathrm{~mm}]}\end{array}$ & $\begin{array}{c}L \\
{[\mathrm{~mm}]}\end{array}$ & $\begin{array}{c}f_{\mathrm{g}} \\
{[\mathrm{kHz}]}\end{array}$ & $\begin{array}{c}\text { Messbereich } \\
{[\mathrm{MHz}]}\end{array}$ & $\begin{array}{c}\text { Messfehler } \\
\Delta \alpha / \alpha[\%]\end{array}$ \\
\hline plan-konkav $^{a}$ & 1 & 40 & $\infty / 2.0$ & 35 & 19 & 40 & $0.2-1.8$ & $5-10(30)^{e}$ \\
bikonkav $^{b}$ & 1.1 & 40 & $2.0 / 2.0$ & 35 & 19 & 39.5 & $0.1-1.4$ & $5-50$ \\
$\begin{array}{l}\text { plan-plan } \\
\text { (bikonkav) }^{c}\end{array}$ & 8 & 10 & $\begin{array}{c}\infty / \infty \\
(4.0 / 4.0)\end{array}$ & 8.4 & 5.5 & 135 & $0.4-12.0^{d}$ & $5-10$ \\
\hline
\end{tabular}

Tabelle 3.1: Resonatordaten. $f_{\mathrm{q}}$ : Quarzgrundresonanzfrequenz, $R_{\mathrm{q}}$ : Wandlerradius, $R_{\mathrm{K}}$ : Krümmungsradius ( ${ }^{a}$ geschliffen, ${ }^{b}$ konkav geformte Spiegel, externe Schallankopplung durch piezoelektrische Keramiken, ${ }^{c}$ gequetscht), $R$ : Zellradius, $L$ : Zelllänge, $f_{\mathrm{g}}:$ Zellgrundresonanzfrequenz, ${ }^{d}$ für Substanzen mit hoher Dämpfung verkleinert sich der Messbereich, ${ }^{e}$ mit Teflonberandung.

Die Resonatoren unterscheiden sich in erster Linie durch den Auf- und Einbau ihrer Wandler sowie in der elektroakustischen Ankopplung. Beim plan-konkaven Resonator ist einer der Wandler mit definiertem Wandlerradius geschliffen. Der 8-MHz Resonator besitzt plane Wandler, die aber beim Einbau leicht verspannt werden, so dass sie eine konkave Form annehmen. Beim 1.1-MHz-Spiegelresonator ist der in Abschnitt 3.2.1.1 geschilderte Resonatoraufbau etwas abgeändert: Hier werden die Stirnseiten des Resonators durch konkav geformte Spiegel abgeschlos- 
sen, die als Reflektoren wirken. Die Schallerzeugung erfolgt extern über eine piezoelektrische Keramik, die mit der Sendereflektorrückseite leicht in Kontakt gebracht wird. Der Schallempfänger wird jedoch kontaktlos durch die Luft betrieben. Dadurch wird vermieden, dass zusätzlich auftretende Reflektorschwingungen mitübertragen werden und die zu messende Zellresonanz verdecken. Detaillierte Beschreibungen der verwendeten Resonatoren finden sich in [22, 31, 35].

\subsubsection{Fehler der Resonatormessung}

Im Folgenden werden einige wesentliche Fehlerquellen des Resonatorverfahrens beschrieben. Eine Abschätzung des relativen Gesamtfehlers für die verschiedenen Apparaturen findet sich in Tab. 3.1.

Fehler in der Frequenz Der Fehler in der Frequenz ist bei den verwendeten Synthetisiersendern vernachlässigbar klein.

Befüllung Luftbläschen in der Resonatorzelle und schlecht benetzte Wandleroberflächen können maßgeblich zu einer Erhöhung der gemessenen Halbwertsbreite führen. Gerade bei den untersuchten polymeren Substanzen, die durch ihre hohe Viskosität stabilisierend auf einmal entstandene Luftblasen wirken, ist dies ein Problem. Durch Entgasen der Substanzen vor der Messung und sehr langsames, zum Teil mehrfaches Befüllen der Resonatorzellen von unten, wurde versucht, die Messbedingungen zu optimieren.

Häufig kann eine schlechte Befüllung an einem ungewöhnlich hohem Halbwertsbreitenminimum erkannt werden. Bei unbekannten Messsubstanzen ist ein solcher Fehler jedoch oft erst nach der Messung durch einen Vergleich mit Daten anderer Messzellen festzustellen. Die Messung muss dann wiederholt werden.

Temperaturschwankungen während der Messung Auch nach einer Thermostatierzeit von mehreren Stunden ist eine leichte Temperaturdrift nicht zu vermeiden. Sie ist mit einer fortschreitenden leichten Verschiebung der Resonanzfrequenz verbunden. Da das Ausmessen der Resonanzen von tiefen zu hohen Frequenzen einige Sekunden in Anspruch nimmt, wird hierdurch die Halbwertsbreite je nach Driftrichtung der Resonanzfrequenz zu groß oder zu klein bestimmt. Vor allem bei niedrigen Dämpfungen und geringen Halbwertsbreiten, wie sie im unteren Frequenzbereich des 1.1-MHz-Spiegel- und 1-MHz-plan-konkav-Resonators auftreten, kann dieser Effekt die Messergebnisse spürbar beeinflussen. Vor Beginn der eigentlichen Messung wurde daher eine Driftmessung im Halbwertsbreitenminimum durchgeführt. Die Messung wurde erst gestartet, nachdem die Driftgeschwindigkeit unter $0.03 \mathrm{~Hz} / \mathrm{s}$ lag (beim 1.1-MHz-Resonator liegt die Driftgeschwindigkeit durch das temperaturstabilisierende große Flüssigkeitsvolumen bei nur etwa $0.003 \mathrm{~Hz} / \mathrm{s}$ ).

Die Temperaturschwankungen während der gesamten Resonatormessung waren nicht größer als $\pm 0.02^{\circ} \mathrm{C}$. Der damit einhergehende Fehler kann aus der Temperaturabhängigkeit des Absorptionskoeffizienten und der Schallgeschwindigkeit für 
die jeweilige Messsubstanz abgeschätzt werden. Er liegt für die untersuchten Substanzen im Promille-Bereich und ist daher vernachlässigbar.

Beim 1-MHz- und 8-MHz-Resonator sind Bohrungen für den Fühler in der Zellberandung vorhanden, so dass die Temperatur unmittelbar in der Nähe des Flüssigkeitsvolumens gemessen werden kann. Beim 1.1-MHz-Spiegelresonator konnte der Fühler mangels Bohrung nur zwischen Außen- und Innenthermostatiermantel geklemmt werden. Dadurch kann der Fehler in der gemessenen Temperatur etwas größer sein.

Eine signifikante Erwärmung der Flüssigkeit durch die eingestrahlte Schallwelle ist wegen der verwendeten sehr geringen Schallleistung nicht zu erwarten.

Referenzverfahren Identische Voraussetzungen bezüglich Temperatur und Resonatorzustand während der Referenz- und Substanzmessung sind ausschlaggebend für eine richtige Bestimmung der Schallgeschwindigkeit und des Absorptionskoeffizienten. Die Messtemperatur durfte daher bei Referenz- und Substanzmessung nicht mehr als $0.02{ }^{\circ} \mathrm{C}$ von der Solltemperatur abweichen. Beide Messungen wurden möglichst zeitnah ausgeführt, um zu vermeiden, dass sich durch mechanische Spannungen die apparativen Eigenschaften des Resonators zwischenzeitlich zu sehr verändern.

Wie zuvor diskutiert, führen Unterschiede von Referenz und Substanz in Dichte, Schallgeschwindigkeit und Absorptionskoeffizient zu Änderungen des Resonatorübertragungsverhaltens. Die Zusatzverluste werden in diesem Fall durch die Gleichungen (3.15) und (3.16) nicht mehr korrekt bestimmt. Der dadurch entstehende Fehler nimmt zu kleineren Frequenzen hin zu, weil dann die wachsenden Beugungsverluste gegenüber der kleiner werdenden Flüssigkeitsdämpfung immer mehr an Bedeutung gewinnen. Durch Auswertung eines Teils der Daten mit verschiedenen Referenzen wurde versucht, systematische Fehler bei der Interpretation der Spektren aufgrund der unterschiedlichen Flüssigkeitseigenschaften zu vermeiden.

Fehler bei der Bestimmung der Schallgeschwindigkeit Durch Vergleich der Ergebnisse aus Messungen an verschiedenen Resonatoren und anschließenden Pulszellen wird der Fehler bei der Bestimmung der Schallgeschwindigkeit auf wenige Promille geschätzt.

Fehler bei der Anpassung der Übertragungsfunktion Die Fehler bei der Anpassung der Halbwertsbreite sind bei kleiner Nebenmodenanzahl und gut auflösbaren Resonanzen gering. Unterhalb von $3 \mathrm{MHz}$ (bei hochdämpfenden Polymeren) bzw. unterhalb von etwa $8 \mathrm{MHz}$ (bei wasserähnlichen Substanzen) werden sie auf etwa $1 \%$ geschätzt. Oberhalb dieses Messbereichs des 8-MHz-Resonators treten jedoch zahlreiche Nebenmoden auf, die sich der Hauptmode überlagern und zum Teil kaum noch aufösbar sind. Trotz einer vorsichtigen schrittweisen Anpassung kann es daher zu einer fehlerhaften oder unvollständigen Berücksichtigung der Neben- 
moden und damit zu einer fehlerhaften Bestimmung des Absorptionskoeffizienten kommen.

\subsubsection{Schwingungspulstransmissionsverfahren}

\subsubsection{Messprinzip}

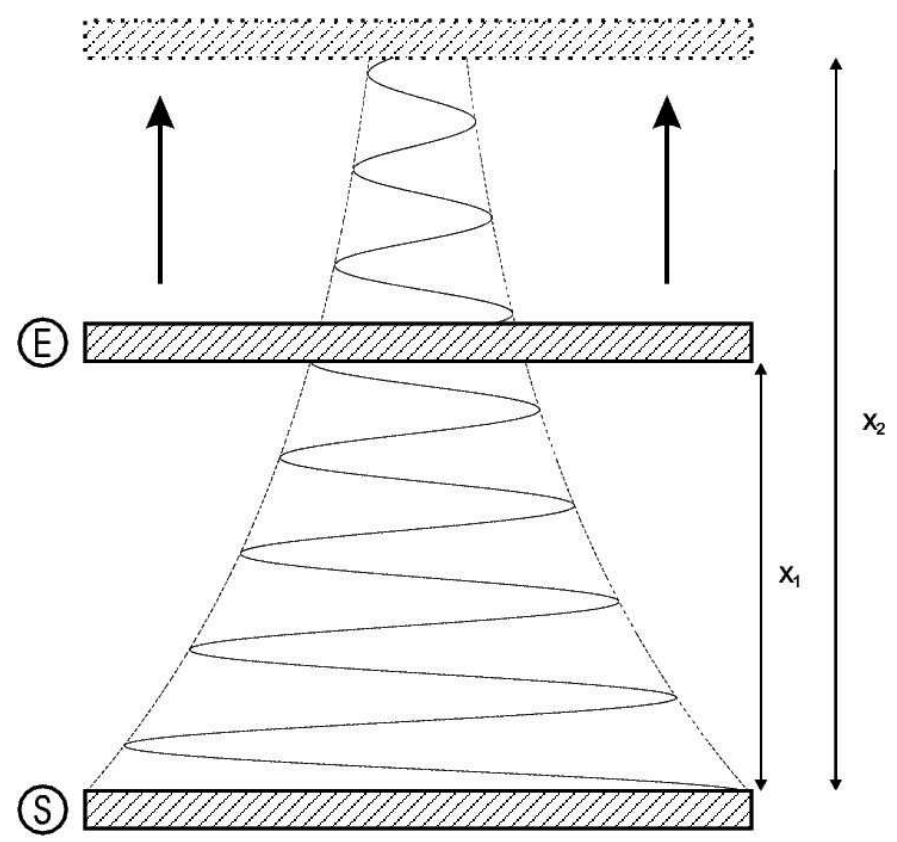

Abbildung 3.4: Prinzip des Transmissionsverfahrens [36]: (S) Sendewandler, (E) beweglicher Empfangswandler

Pulstransmissions- und Resonatorverfahren beruhen auf der gleichen Grundanordnung. Auch beim Pulstransmissionsverfahren befindet sich die Messflüssigkeit zwischen zwei piezoelektrischen Wandlern, von denen einer zur Erzeugung und ein zweiter zur Detektion des Wellenfeldes eingesetzt wird. Im Gegensatz zum Resonatorverfahren ist hier jedoch der Empfangswandler entlang der Wandlernormalen beweglich angeordnet (Abb. 3.4). Durch kontinuierliches Variieren des Wandlerabstands kann so, bei konstanter Frequenz $f$, die Amplitude des Schalldrucks in Abhängigkeit von der Dicke $x$ der durchlaufenen Flüssigkeitsschicht gemessen werden. Dabei treten jedoch einige Schwierigkeiten auf, die im Folgenden beschrieben werden.

Unter der Voraussetzung, dass der Sendewandler ebene Wellen der Wellenlänge $\lambda$ abstrahlt, die an den Wandleroberflächen mit einem Reflexionsfaktor $r$ reflektiert werden, erhält man bei kontinuierlicher sinusförmiger Anregung die Übertragungsfunktion $T(x)$ - analog zum Resonatorverfahren - als Verhältnis der am Empfangswandler abgenommenen Spannung $U_{\mathrm{E}}$ und der am Sendewandler einge- 
speisten Spannung $U_{\mathrm{S}}$ :

$$
T(x)=\frac{U_{\mathrm{E}}}{U_{\mathrm{S}}}=\hat{T} \frac{(1+r) e^{-\gamma x}}{1-r^{2} e^{-2 \gamma x}}+A_{\mathrm{u}}(x) e^{\phi_{\mathrm{u}}} .
$$

$\gamma=\alpha+i \frac{2 \pi}{\lambda}$ bezeichnet hier wieder die Ausbreitungskonstante, $\hat{T}$ erfasst die Umsetzung von akustischer in elektrische Leistung und umgekehrt. Für $r=1$ und $x=L$ geht der erste Summand in die bereits diskutierte Übertragungsfunktion des idealen Resonators (3.11) über. Der zweite Summand berücksichtigt das elektrische Übersprechen. An die gemessene Übertragungsfunktion wäre also die komplizierte Funktion (3.23) anzupassen, wobei die nicht bekannten Größen $r$ und $A_{\mathrm{u}} / \hat{T}$ als zusätzliche freie Parameter bestimmt werden müssten. Ein weiteres Problem wird durch das Übersprechen verursacht: Wegen der ineffizienten Energiekonversion der Wandler kann es in ungünstigen Fällen vorkommen, dass das Nutzsignal nur einen Bruchteil des Übersprechens ausmacht, wodurch die Messung sehr ungenau würde. Im Gegensatz zur Phase $\phi_{\mathrm{u}}$, die wegen der großen Wellenlänge des elektromagnetischen Signals im betrachteten Frequenzbereich hinreichend unabhängig von $x$ ist, kann die Amplitude $A_{\mathrm{u}}$ außerdem abstandsabhängig sein, was ebenfalls zu Fehlern bei der Bestimmung von $\alpha$ führen kann.

Um diese Schwierigkeiten zu umgehen, wird beim Schwingungspulstransmissionsverfahren anstatt eines kontinuierlichen Signals eine pulsmodulierte harmonische Welle angeregt. Dadurch können bei genügend kurzen Pulsen die mehrfach reflektierten Signale und das interessierende, direkt übertragene primäre Messsignal auf Grund ihrer unterschiedlichen Laufzeiten zeitlich voneinander getrennt werden. Auch das nahezu verzögerungsfrei übertragene elektrische Übersprechen kann auf diese Weise vom Primärpuls separiert werden. Eine zusätzliche Verbesserung der zeitlichen Trennung wird durch den Einbau von akustischen Verzögerungsleitungen zwischen Wandler und Flüssigkeit erreicht, die bei hohen Frequenzen und kleinen Messabständen die Laufzeit des akustischen Signals erhöhen. Bei ausreichender Trennung des Primärpulses von den Mehrfachreflexionen und dem elektrischen Übersprechen vereinfacht sich der Betrag der Übertragungsfunktion (3.23) $\mathrm{zu}$

$$
|T(x)| \sim e^{-\alpha x},
$$

aus der durch Anpassung einer einfachen Exponentialfunktion der Absorptionskoeffizient bestimmt werden kann.

Mehrfachreflexionen bei kleinen Wandlerabständen Ausreichende Trennung von mehrfach reflektierten Wellenpulsen und Primärsignal liegt dann vor, wenn der Startabstand, d.h. der minimale Wandlerabstand, $x_{\text {start }}>\tau c_{\mathrm{s}} / 2$ gewählt wird, wobei $\tau$ die Pulsdauer bezeichnet. Unterschreitet man diesen Startabstand, überlagert sich dem exponentiellen Abfall des Signals eine Welligkeit. Sie ergibt sich aus dem Beitrag $r^{2} e^{-2 \gamma x}$ im Nenner der Übertragungsfunktion (3.23), der 


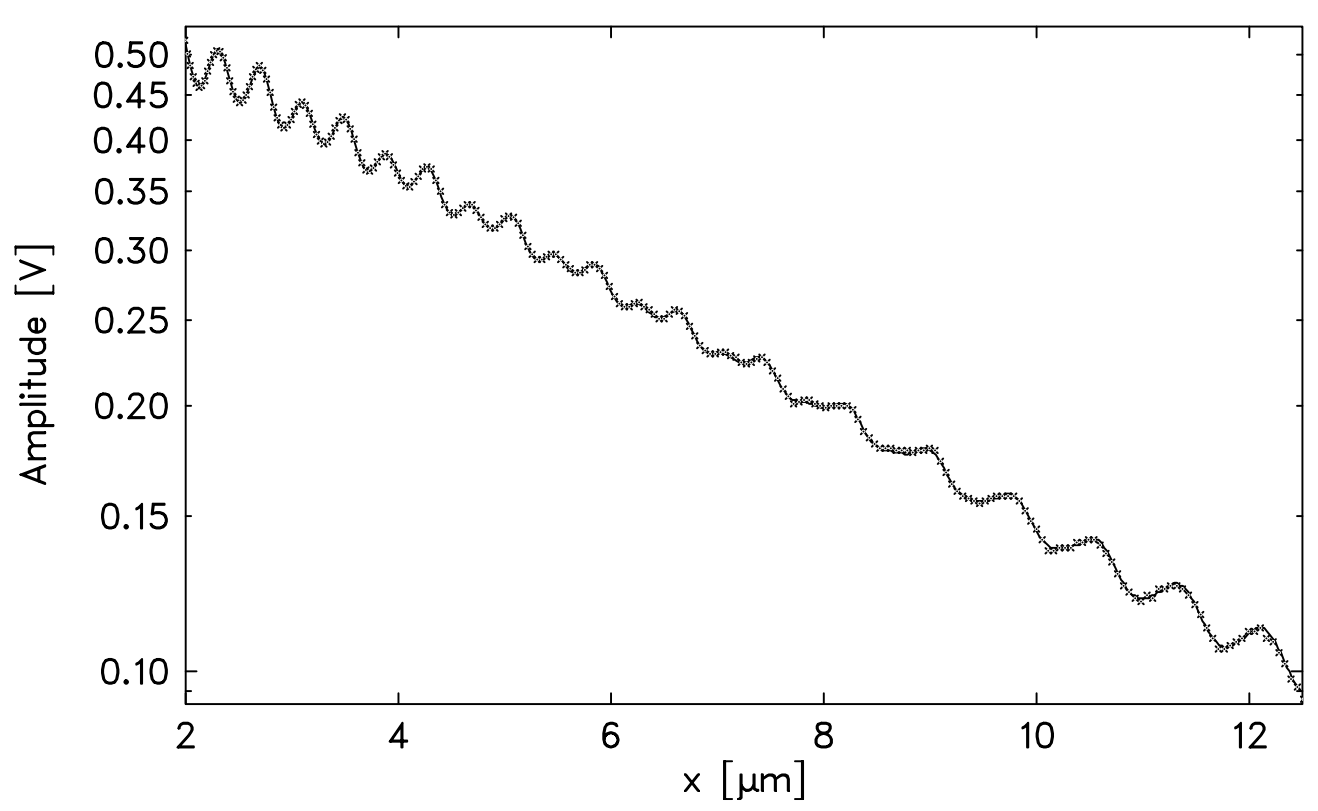

Abbildung 3.5: Gemessene Spannungsamplitude $(\times)$ als Funktion des Wandlerabstands, gemessen für eine N-Propyl-Harnstoff/Wasser-Lösung bei ca. $2 \mathrm{GHz}$ und $25^{\circ} \mathrm{C}$ mit der PZT-Zelle [37]. Durchgezogene Kurve: Angepasste, nach Gl. (3.23) gebildete, Betragsfunktion $|T(x)|$.

den Einfluss der Mehrfachreflexionen erfasst. Unter der Voraussetzung, dass Übersprechen und akustisches Signal zeitlich voneinander getrennt sind, erhält man in diesem Fall für den Betrag der Übertragungsfunktion

$$
|T(x)|=\hat{T} \frac{(1+r) e^{-\alpha x}}{\sqrt{1+r^{4} e^{-4 \alpha x}+2 r^{2} e^{-2 \alpha x} \cos (2 \beta x)}} .
$$

Die durch die Cosinus-Funktion beschriebene Welligkeit pflanzt sich mit einer Wellenlänge von $\lambda / 2$ fort und klingt mit $e^{-2 \alpha x}$ ab. Für $\alpha x>3$ spielt sie praktisch keine Rolle, für $x_{\text {start }}<\tau c_{\mathrm{s}} / 2$ und $\alpha x<3$ muss sie jedoch bei der Anpassung mitberücksichtigt werden. Aus ihrer Wellenlänge lässt sich die Schallgeschwindigkeit in der Flüssigkeit bestimmen, die für die richtige Nachführung des Abtastpulses bei Abstandsänderung benötigt wird (vergl. Abschnitt 3.2.2.2).

Einfluss des Übersprechens bei hohen Frequenzen Bei sehr hohen Frequenzen $(>1000 \mathrm{MHz})$ gelingt die vollständige Separation von Übersprechen und akustischer Übertragungsfunktion oft nicht mehr. Hier kann das Übersprechen zusammen mit dem Nutzsignal bei der Betragsbildung in (3.23) zu einem Kreuzterm führen, der proportional zu $\cos \left(\beta x+\phi_{\mathrm{u}}\right)$ verläuft und somit eine $\lambda$-Welligkeit verursacht. Diese kann bei PZT- und Hyperschallmesszelle (vergl. Abschnitte 3.2.2.2 und 3.2.2.3) mit angepasst werden.

Abb. 3.5 zeigt einen typischen abstandsabhängigen Verlauf der Spannungsamplitude bei ca. $2 \mathrm{GHz}$ und $25^{\circ} \mathrm{C}$, gemessen mit der PZT-Zelle. Gut zu erkennen ist der Übergang von der $\lambda / 2$ - in die $\lambda$-Welligkeit. 
Beugungskorrektur Bis hierher wurde davon ausgegangen, dass der Sendewandler ebene Wellen abstrahlt. Aufgrund der endlichen Wandlerradien kommt es jedoch zu Beugungserscheinungen. Sie führen zu einer Aufweitung des Schallfeldes, so dass mit zunehmendem Wandlerabstand ein wachsender Teil der eingestrahlten Schallleistung nicht auf den Empfangswandler übertragen wird. Um den Absorptionskoeffizienten nicht systematisch zu groß zu messen, werden die Messwerte nach einer auf einem Vorschlag von Fay [38] basierenden und von Menzel [39] modifizierten empirischen Formel korrigiert:

$$
|T(x)| \sim e^{-\alpha x} \cdot\left(e^{-\sqrt{\frac{x}{\beta A}}}\right)^{g(x)}
$$

$A$ bezeichnet dabei die Wandlerfläche, $g(x)=1-x / 100 \mathrm{~mm}$ einen empirisch bestimmten Gewichtsexponenten. Die Korrektur gewinnt vor allem bei Messfrequenzen unterhalb von $30 \mathrm{MHz}$ an Bedeutung.

\subsubsection{Apparativer Aufbau}

Insgesamt wurden bei diesem Verfahren fünf verschiedene Messzellen verwendet, die sich je nach Messbereich, für den sie konstruiert wurden, in ihrer Bauart etwas unterscheiden (s. Tab. 3.2). Entsprechend wurde auch der Aufbau der Messplätze in der Zusammensetzung der Geräte den jeweiligen Bedingungen angepasst. Der grundsätzliche Aufbau ist jedoch bei allen Zellen derselbe.

Abb. 3.6 zeigt das Blockschaltbild eines solchen Messplatzes. Das Signal eines Synthetisiersenders (1) wird mit Hilfe eines PIN-Diodenschalters bzw. zweier Dioden-Ringmischer (2) mit dem Signal eines Rechteckpuls-Generators (3) moduliert und anschließend verstärkt (4). Über zwei rechnergesteuerte Koaxialrelais kann das Signal in verschiedene Zweige übertragen werden. Um die Leistung von Mess- und Referenzzweig anzupassen, durchläuft das Signal im Messzweig zunächst ein variables Dämpfungsglied oder ein Anpassungsglied mit Kurzschlussstichleitungen (6), bevor es zum Sendewandler (7a) der Messzelle (7) gelangt. Dort wird eine Ultraschallwelle erzeugt, die die zu untersuchende Flüssigkeit durchläuft und von einem identisch gebauten Empfangswandler detektiert wird. Durch einen vom PC (22) kontrollierten Schrittmotor $(17,18)$ kann der Abstand der Wandler variiert werden. Zur Bestimmung des Abstandes wird ein optischer Längentaster (19) (Heidenhain CT 25, MT 25, MT60) verwendet. Bei der PZT-Zelle wird die Abstandsänderung von einem Piezo-Translator (Physik Instrumente, Waldbronn) übernommen, die Abstandsmessung erfolgt mit einem induktiven Längentaster (LVDT, Schlumberger), der zuvor gegen einen optischen Längentaster (Heidenhain, MT 12) kalibriert wird.

Im Referenzzweig trifft das Sendersignal auf ein Cut-Off-Dämpfungsglied (8), das der Bestimmung der nichtlinearen Kennlinie aller Bauteile (10-14) dient, die sich an Mess- und Referenzzweig anschließen. Es besteht im Wesentlichen aus einem Hohlleiter variabler Länge, der unterhalb seiner Grenzfrequenz betrieben wird 


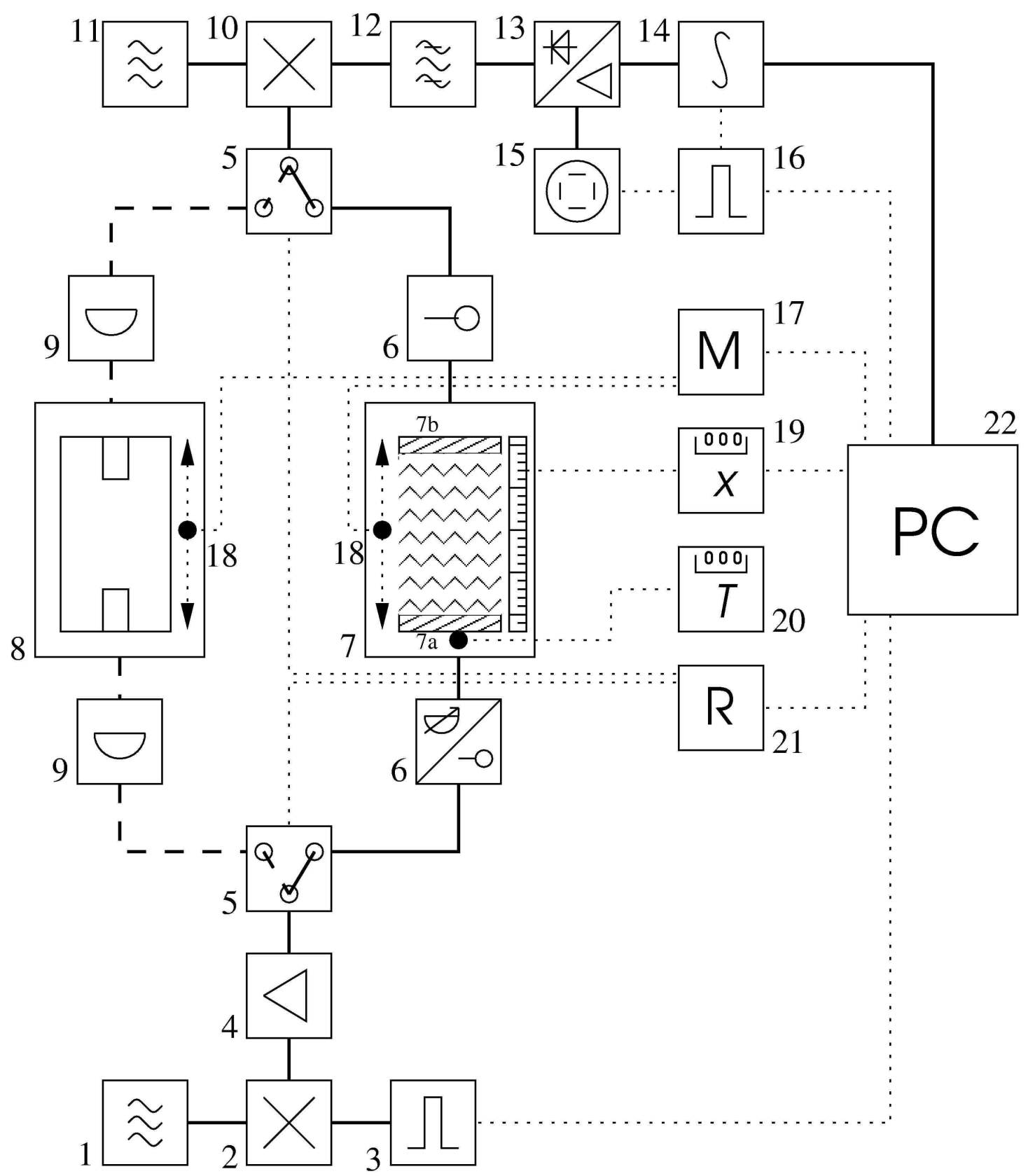

Abbildung 3.6: Blockschaltbild der Pulstransmissionsmessplätze [27]: (Durchgezogene Linie: Signalleitung durch den Messzweig, gestrichelte Linie: Signalweg durch den Referenzzweig, gepunktete Linien: Steuerleitungen). (1) Synthetisiersender, (2) Modulator, (3) Pulsgenerator, (4) Verstärker, (5) Koaxialrelais, (6) Amplitudenregelung und Impedanzanpassung (variables Dämpfungsglied, Kurzschlussstichleitungen), (7) Messzelle mit Sende- (7a) und Empfangswandler (7b), (8) Cut-Off-Dämpfungsglied, (9) Dämpfungsglied zur Impedanzanpassung, (10-13) Überlagerungsempfänger, (14) Sample-and-Hold-Schaltung und A/D-Wandler, (15) Oszillograph, (16) Pulsgenerator, (17) Motorsteuerung oder D/A-Wandler mit $1 \mathrm{kV}$-Verstärker, (18) Schrittmotor oder Piezo-Translator, (19) optischer oder induktiver Längentaster zur Abstandsmessung, (20) Digitalthermometer, (21) Relaistreiberkarte, (22) Prozessrechner. 
und dadurch bei fester Messfrequenz zu einer von der Hohlleitergeometrie abhängigen exponentiellen Schwächung des Signals führt [40]. Die Länge des Rundhohlleitersegments wird durch einen weiteren rechnergesteuerten Schrittmotor $(17,18)$ eingestellt.

Nach dem Durchlaufen der Messzelle bzw. des Cut-Off-Dämpfungsgliedes gelangt das Signal zu einem Überlagerungsempfänger (10-13), der das Signal mittels eines Hilfsoszillators (11) auf eine Zwischenfrequenz mischt (10), filtert (12), demoduliert und verstärkt (13). Danach wird das Signal auf einem Oszillographen (15) dargestellt und über eine Sample-and-Hold-Schaltung und A/D-Wandlerkarte (14) integriert und vom PC eingelesen.

Nach Abgleich und Optimierung des Signals in Mess- und Referenzzweig für die jeweilige Messfrequenz läuft die Messung automatisch ab. Der Rechner liest die Daten der Längentaster aus und steuert Sendepulsgenerator, A/D-Wandler, Oszillograph sowie das mit Hilfe eines Pulsgenerators (16) erzeugte Triggersignal der Sample-and-Hold-Schaltung. Dabei wird der Triggerzeitpunkt entsprechend der Laufzeitverschiebung des Messpulses durch die Änderung des Wandlerabstands so nachgeführt, dass die Messung stets an der gleichen Stelle des Messpulses stattfindet. Hierzu wird vor jeder Messung die Schallgeschwindigkeit aus der Welligkeit der Übertragungsfunktion bei kurzen Wandlerabständen bestimmt.

Um Temperaturschwankungen in der Flüssigkeit zu reduzieren, werden die einzelnen Wände der Messzelle thermostatiert. Zusätzlich befindet sich die Zelle in einer thermostatierten Kammer. Mittels eines PT-100 Digitalthermometers (20) kann die Temperatur an der inneren Wand des Messzylinders kontrolliert werden.

Kennlinie des Empfangssystems Die am Empfangswandler detektierte Spannung $U_{\mathrm{E}}(x)$ wird durch Demodulation und Verstärkung innerhalb des Empfangssystems nicht vollständig linear verarbeitet. Am A/D-Wandler wird daher nicht die Spannung $U_{\mathrm{E}}$ registriert, sondern eine von $U_{\mathrm{E}}$ abhängige Spannung $U_{\text {mess }}(x)$. Den Zusammenhang zwischen $U_{\mathrm{E}}$ und $U_{\text {mess }}$ beschreibt die Kennlinie $K$ des Empfangssystems.

$$
U_{\text {mess }}(x)=K\left(U_{\mathrm{E}}(x)\right)
$$

Bei bekannter Kennlinie lässt sich aus ihrer Umkehrfunktion die gesuchte Spannung am Empfangswandler in Abhängigkeit vom Wandlerabstand bestimmen. Der Verlauf dieser Kennlinie wird für jede Messfrequenz mit Hilfe des Cut-Off-Dämpfungsgliedes ermittelt [40].

\subsubsection{Die verwendeten Pulstransmissionszellen}

Für die Messungen dieser Arbeit wurden die in Tab. 3.2 aufgelisteten Pulszellen verwendet. Ihr detaillierter Aufbau ist in der angegebenen Literatur beschrieben. Die angegebenen zu erwartenden relativen Fehler im Absorptionskoeffizienten beruhen auf eigenen Messungen an polymeren Substanzen sowie auf Erfahrungen anderer [22, 31, 37]. Allgemein ist bei hochdämpfenden Substanzen, wie sie in dieser Arbeit untersucht wurden, zu hohen Frequenzen hin mit größeren Messfehlern 


\begin{tabular}{l|ccccc} 
Pulszelle & tieffrequent (I) & tieffrequent (II) & hochfrequent & Hyperschall & PZT \\
& & & & & \\
Wandler & Quarz & Quarz & LiNbO $_{3}$ & LiNbO $_{3}$ & ZnO \\
VZ & ja & nein & ja & nein & ja \\
$R_{\mathrm{q}}[\mathrm{mm}]$ & 30 & 20 & 6 & 1.5 & 3.5 \\
$f_{\mathrm{q}}[\mathrm{MHz}]$ & 1.0 & 1.0 & 10 & breitbandig & ca. 1300 \\
$f_{\min }[\mathrm{MHz}]$ & 3 & 3 & 30 & 550 & 550 \\
$f_{\max }[\mathrm{MHz}]$ & 100 & 100 & 530 & 2000 & 4600 \\
$\tau[\mu \mathrm{s}]$ & $4-8$ & $4-8$ & $2-4$ & 1.5 & 1.5 \\
Taster & optisch & optisch & optisch & optisch & induktiv \\
& $(\mathrm{MT} 60)$ & $(\mathrm{MT} 60)$ & $(\mathrm{MT} 25)$ & $(\mathrm{CT} 25)$ & $(\mathrm{LVDT})$ \\
$x_{\min }[\mathrm{nm}]$ & 125 & 125 & 125 & 0.5 & 8 \\
$x_{\max }[\mathrm{mm}]$ & 55 & 55 & 25 & 1 & 0.04 \\
$V[\mathrm{ml}]$ & ca. 200 & ca. 130 & ca. 10 & ca. 3 & ca. 0.5 \\
Literatur & {$[41]$} & {$[42]$} & {$[43]$} & {$[44],[39]$} & {$[44],[23]$} \\
& & & & & \\
\hline & & & & & \\
$\Delta \alpha / \alpha[\%]$ & $1.0-3.0$ & $0.5-3.0$ & $0.5-1.5$ & $1.0-4.0$ & $1.0-4.0$
\end{tabular}

Tabelle 3.2: Die Schwingungspulstransmissionsmesszellen. VZ: Verzögerungsleitungen ( ${ }^{a}$ Stab wirkt selbst als Verzögerungsleitung), $R_{\mathrm{q}}$ : Wandlerradius, $f_{\mathrm{q}}$ : Wandlergrundresonanzfrequenz, $f_{\min }$ : tiefste mögliche Messfrequenz, $f_{\max }$ : höchste mögliche Messfrequenz (bei geringer Dämpfung und optimalem Zustand der Apparatur), $\tau$ : Pulslänge, $x_{\min }$ : kleinste mögliche Abstandsänderung (Schrittweite), $x_{\max }$ : maximale Abstandsänderung (Fahrweg des Längentasters ist begrenzt), $V$ : Flüssigkeitsvolumen, $\Delta \alpha / \alpha$ : relativer Fehler des gemessenen Absorptionskoeffizienten.

zu rechnen, da die Messungen dann bei extrem kurzen Wandler-Startabständen und Messstrecken durchgeführt werden müssen (Beispiel: Hyperschallmesszelle, Messfrequenz $1850 \mathrm{MHz}, x_{\text {start }}=1.5 \mu \mathrm{m}, x_{\text {mess }}=2.7 \mu \mathrm{m}$ für PPG 1000). Die angegebene maximale Messfrequenz der einzelnen Zellen, die sich auf Substanzen mit geringer Absorption bezieht, reduziert sich daher zum Teil drastisch.

Ein prinzipieller Unterschied der Messzellen liegt in der Art der Ultraschallerzeugung bzw. -detektion. Bei den beiden tieffrequenten und der hochfrequenten Pulszelle erfolgt die Schallwandlung durch Anregung von Dickenschwingungen in Quarz bzw. $\mathrm{LiNbO}_{3}$-Kristallen, wobei die ungeradzahligen Vielfachen der Grundresonanzfrequenz als Messfrequenzen genutzt werden können.

Für die PZT-Zelle dient eine $1 \mu \mathrm{m}$ dicke ZnO-Schicht als Wandlermaterial, die im Anschluss an eine $0.1 \mu \mathrm{m}$ dicke Gold-/Chromschicht auf eine Saphir-Verzögerungsleitung gesputtert wurde [44]. Im Gegensatz zu den unterhalb von $500 \mathrm{MHz}$ verwendeten Dickenschwingern aus dünnen Einkristallen hat man das System ZnOGold-Saphir insgesamt als Resonanzsystem zu betrachten, das durch die verschiedenen Materialien und deren Kopplung die breite Grundresonanz der ZnO-Schicht (bei ca. $3 \mathrm{GHz}$ ) verschiebt und verformt. Gute Übertragungseigenschaften wurden empirisch bei Frequenzen von 0.8-2.6 GHz mit einem relativen Maximum in der Signalübertragung bei $1.3 \mathrm{GHz}$ und im Bereich 3.7-4.6 GHz mit einem Maximum bei 3.9 GHz gefunden, so dass man die Grund-Resonanzfrequenz des Gesamtsystems 
bei $1.3 \mathrm{GHz}$ vermuten kann.

Bei der Hyperschallzelle wird ein anderes Verfahren zur Schallerzeugung genutzt. Hier wird ein etwa $10 \mathrm{~mm}$ langer piezoelektrischer $\mathrm{LiNbO}_{3}$-Stab mit einem Durchmesser von $3 \mathrm{~mm}$ als Wandler verwendet, der nach dem Prinzip der Oberflächenanregung von Bömmel und Dransfeld [45] über einen Koaxialresonator angeregt wird. Die Schalldetektion erfolgt reziprok. Da die Koaxialresonatoren mit Hilfe eines Kurzschlussschiebers kontinuierlich eingestellt werden können, sind die Messfrequenzen in einem weiten Bereich frei wählbar.

Zur besseren zeitlichen Trennung von elektrischem Übersprechen und Messsignal sind außer in der PZT-Zelle auch in der hochfrequenten und in der tieffrequenten Pulszelle (I) Verzögerungsleitungen eingebaut.

\subsubsection{Fehler der Pulstransmissionsmessung}

Im folgenden Abschnitt werden wesentliche Fehlerquellen des Pulstransmissionsverfahrens dargestellt. Eine Abschätzung des relativen Gesamtfehlers im Absorptionskoeffizienten findet sich in Tab. 3.2. Da viele Fehler stark von der Messsubstanz abhängen, sind die Angaben auch hier nur als Richtwerte anzusehen.

Fehler in der Frequenz Der Fehler in der Frequenz ist bei den verwendeten Synthetisiersendern vernachlässigbar klein.

Temperaturinstabilitäten Die an der inneren Zellwand gemessene Temperatur wurde während der gesamten Messung auf Solltemperatur $\pm 0.02^{\circ} \mathrm{C}$ konstant gehalten. Der dadurch entstandene Fehler im Absorptionskoeffizienten liegt bei den untersuchten Substanzen im Promille-Bereich und ist damit vernachlässigbar. Größeren Einfluss auf die Messgenauigkeit können Temperaturgradienten in der Flüssigkeit haben, die durch punktuelle Temperaturmessung nicht erfasst werden. Diese können besonders bei den tieffrequenten Messzellen mit ihren großen Flüssigkeitsvolumina auftreten, da hier beim Auseinanderfahren der Wandler größere Mengen Flüssigkeit von der Oberfläche her nachfließen. Dadurch ändert sich möglicherweise der Absorptionskoeffizient während der Messung. Die Anpassung der Messdaten durch eine Exponentialfunktion mit festem $\alpha$ führt dann zu systematischen Fehlern. Da das Messprogramm einen graphischen Vergleich von gemessenen Daten und angepasster Funktion vornimmt, lässt sich dieser Fehler frühzeitig erkennen und durch ausreichend lange Thermostatierzeiten, nicht zu hohe Befüllung der Messzylinder sowie Wiederholungsmessungen weitgehend vermeiden.

Auch bei hohen Frequenzen und damit erhöhter Schallabsorption kann eine signifikante Erwärmung der Flüssigkeit durch die Schallwelle wegen der sehr geringen Schallleistung und der Verwendung pulsmodulierter Wellen ausgeschlossen werden.

Nachführung des Abtastpulses Aufgrund der Übertragungseigenschaften der Wandler ist der akustische Puls gegenüber dem elektronisch angeregten Rechteckpuls an den Flanken abgerundet. Erfolgt die Abtastung des Pulses infolge einer 
ungenauen Nachführung nicht immer an der gleichen Stelle, wird der Absorptionskoeffizient systematisch falsch bestimmt. Da sich dieser Fehler meist in einer Abweichung vom exponentiellen Abklingverhalten bemerkbar macht, kann er durch Vergleich der Messdaten und der angepassten Funktion erkannt und vermieden werden.

Paralleljustierung Bei langen Fahrwegen kann eine mangelhafte Paralleljustierung der Wandler zu einem systematischen Fehler führen, da hierdurch der Empfangswandler zunehmend aus der Achse des Schallfeldes herausfährt und somit eine zu große Absorption gemessen wird. Da sich das Abstrahlverhalten des Wandlers mit der Frequenz ändern kann, ist die Paralleljustierung bei jeder neuen Messfrequenz zu optimieren.

Beugungskorrekturformel Bei niedrigen Frequenzen gilt die Beugungskorrekturformel (3.26) nicht mehr exakt. Vor allem bei Substanzen mit geringer Dämpfung führt dies zu einer größeren Unsicherheit im Absorptionskoeffizienten. 
3 Ultraschallspektroskopie 


\section{Ultraschallabsorptionsspektren der Oligoethylenglykole}

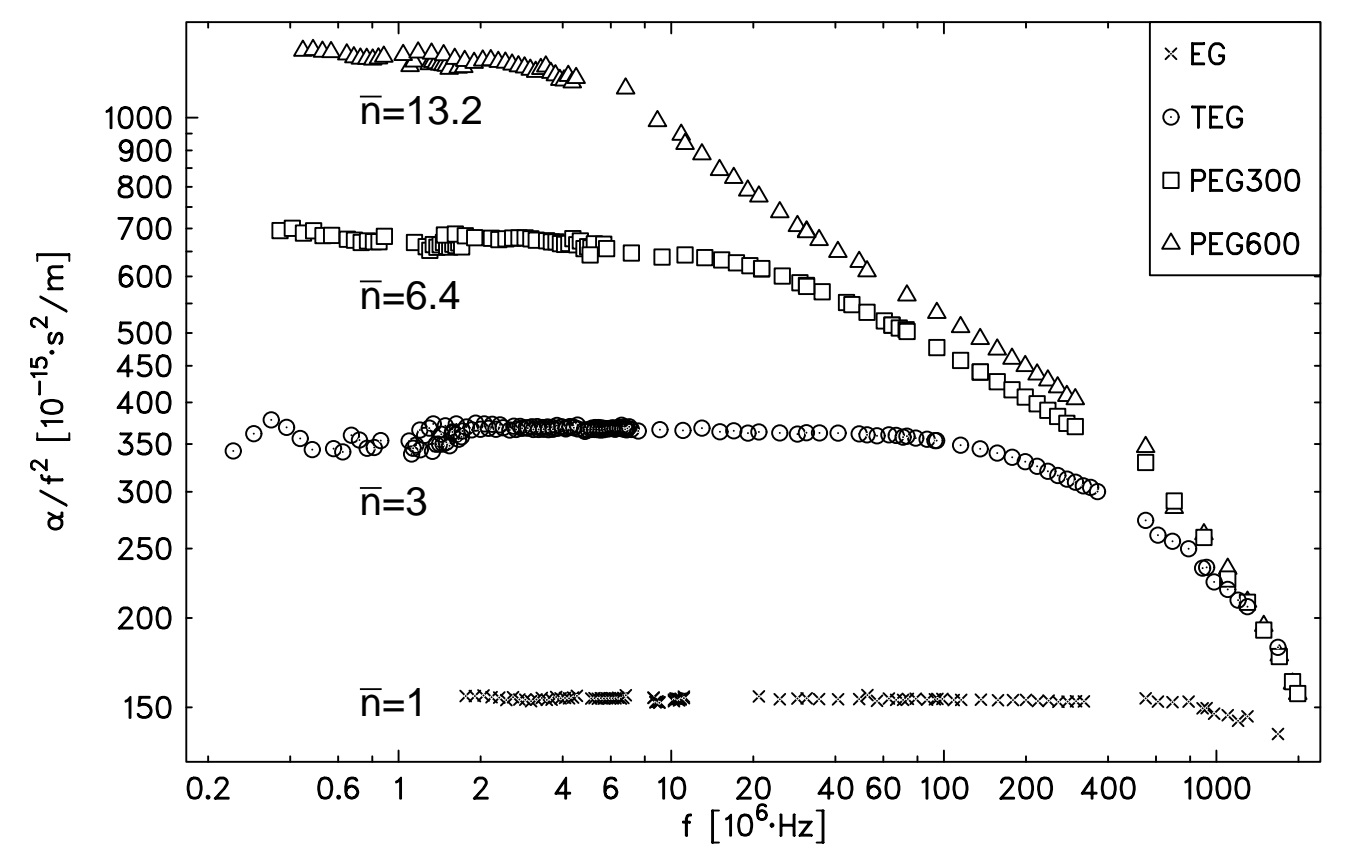

Abbildung 4.1: Ultraschallabsorptionsspektren von vier Oligoethylenglykolschmelzen mit unterschiedlicher Kettenlänge, gemessen bei $25^{\circ} \mathrm{C} . \bar{n}$ : mittlere Kettenlänge.

Abb. 4.1 zeigt die frequenznormierten Absorptionspektren von vier Oligoethylenglykolschmelzen mit unterschiedlichen Kettenlängen, alle gemessen bei $25^{\circ} \mathrm{C}$. Als Referenz wurden bei den Resonatormessungen Harnstoff-Wasser-Lösungen verwendet. Schon diese vergleichsweise kurzkettigen Polymere zeigen sehr breite Absorptionsspektren. Bei allen untersuchten Kettenlängen (zwischen $\bar{n}=1$ und $\bar{n}=13.2$ ) ist im hochfrequenten Teil der Spektren um $1 \mathrm{GHz}$ deutlich ein Relaxationsbereich zu erkennen. Das Ende dieser Relaxationsstufe wird im untersuchten Frequenzbereich nicht erreicht, was die Bestimmung eines frequenzunabhängigen Hintergrundanteils nach Abschnitt 3.1.1.2 bei der späteren Interpretation der Spektren sehr erschwert. Mit wachsender Kettenlänge nimmt die Schallabsorption zu, weitere Relaxationsbereiche deuten sich an. Zu tiefen Frequenzen hin strebt $\alpha / f^{2}$ gegen einen konstanten Wert. Während jedoch bei Ethylenglykol (EG) und Triethylenglykol (TEG) bereits klar ein Plateau erreicht wird, zeigen die tieffrequenten Punkte bei 
PEG 300, PEG 600 und PEG 400 (II)1 noch eine leichte Tendenz, mit abnehmender Frequenz anzusteigen. Abschnitt 4.1 wird sich mit der Frage befassen, ob es sich bei dieser Tendenz um ein Artefakt oder um die Flanke eines tieffrequenten Relaxationsprozesses handelt.

Extrapoliert man den spektralen Verlauf zu tiefen Frequenzen hin, so ist näherungsweise ein linearer Zusammenhang zwischen dem tieffrequenten $\alpha / f^{2}$-Wert $\left(\alpha / f^{2}\right)_{\mathrm{tf}}$ und der mittleren Kettenlänge, d.h. der mittleren Anzahl der monomeren Einheiten pro Molekül, zu beobachten (s. Abb. 4.2). Ein ganz ähnliches

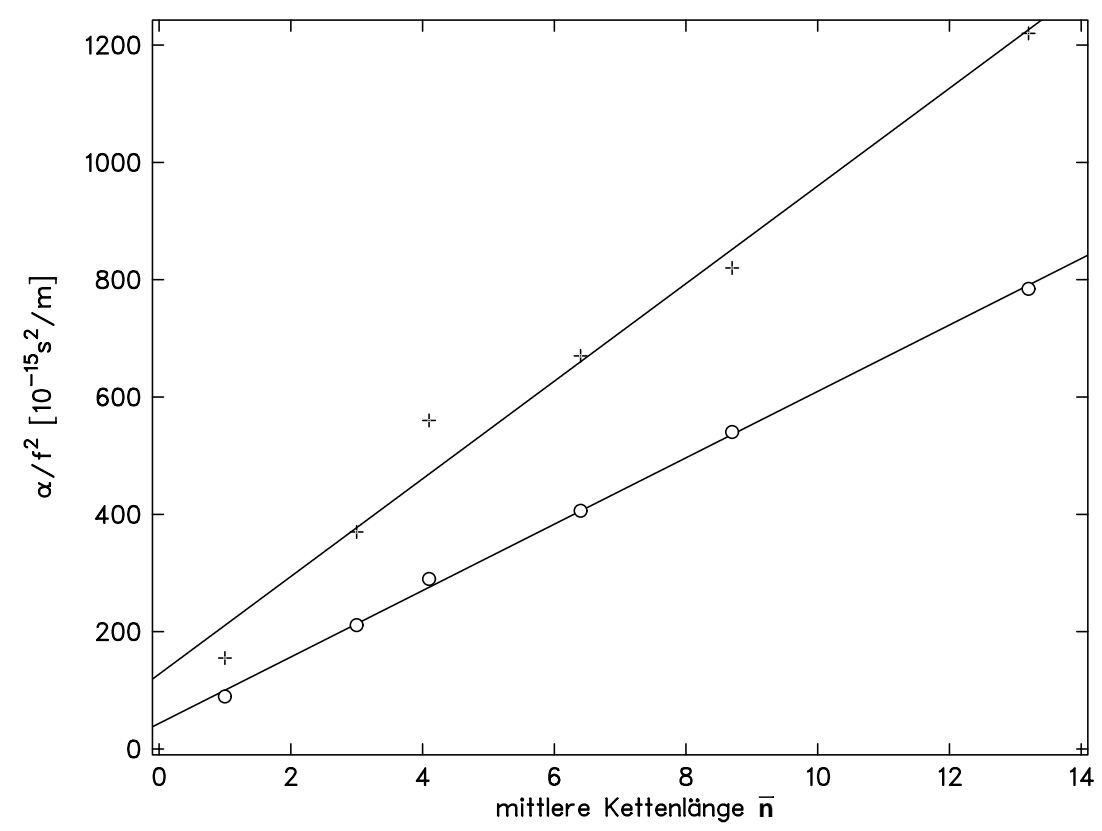

Abbildung 4.2: $\alpha / f^{2}$-Wert $(+)$ der Oligoethylenglykolspektren bei tiefen Frequenzen (um 1.5 MHz) und nach Gl. (3.4) berechneter tieffrequenter Scherviskositätsanteil (o) als Funktion der mittleren Kettenlänge. Messtemperatur $25^{\circ} \mathrm{C}$, durchgezogene Linien beschreiben die durch lineare Regression ermittelten Ausgleichsgeraden.

Verhalten zeigt auch der Scherviskositätsanteil von $\left(\alpha / f^{2}\right)_{\mathrm{tf}}$, der sich mit Hilfe von Gl. (3.4) und den im Anhang dargestellten Ergebnissen der Scherviskositäts-, Schallgeschwindigkeits- und Dichtemessungen berechnen lässt. Auch hier steigt die Absorption linear mit zunehmender Kettenlänge an, die Steigungen beider Geraden unterscheiden sich nur wenig, was auf ein vergleichsweise stabiles Verhältnis $\eta_{\mathrm{s}} / \eta_{\mathrm{v}}$ von Scher- und Volumenviskosität schließen lässt. Eine ausführlichere Diskussion dieses Ergebnisses findet sich in Abschnitt 4.3.3.

Bei Frequenzen um $400 \mathrm{MHz}$ ist in allen gemessenen Spektren eine Messlücke vorhanden. Wegen der starken Flüssigkeitsdämpfung der Polymerschmelzen waren in diesem Frequenzbereich Messungen mit der hochfrequenten 10-MHz-Pulszelle nicht mehr möglich, an der Hyperschall- und PZT-Messzelle finden sich geeignete Messfrequenzen jedoch erst oberhalb von $550 \mathrm{MHz}$.

\footnotetext{
${ }^{1}$ nicht abgebildet
} 
Je nach Flüssigkeitsdämpfung findet sich eine weitere Messlücke im Übergangsbereich zwischen 8-MHz-Resonator und tieffrequenten 1-MHz-Pulszellen. Hohe Absorptionskoeffizienten wie z. B. bei PEG600 schränken den Messbereich des 8-MHz-Resonators zu hohen Frequenzen hin deutlich ein, da hier Haupt- und Nebenmoden nicht mehr getrennt werden können (vergl. Abschnitte 3.2.1.4- 3.2.1.7). Geringe Dämpfungen führen am tieffrequenten Rand des Messbereichs der 1-MHzPulszellen wegen des begrenzten Fahrweges des Empfangswandlers zu einer erhöhten Streuung der Messwerte. Messpunkte, die aus derartigen messtechnischen Gründen nicht in den gesamten Verlauf des Spektrums passten, wurden bei der Interpretation der Spektren nicht berücksichtigt, dazu gehören auch Punkte im Bereich von Scher- und Dickenschwingungsresonanzen der Quarze.

Im Bereich der tieffrequenten Resonatoren ist allgemein eine Zunahme der Streuung mit abnehmender Schallabsorption zu beobachten. Außerdem ist ein kleiner Versatz zwischen den Daten von tief- und hochfrequenten Resonatoren um $1 \mathrm{MHz}$ herum zu erkennen, der ebenfalls mit abnehmender Dämpfung größer wird. Interessant ist dabei, dass die verschobenen Datensätze dennoch parallel verlaufen, die Steigung also erhalten bleibt. Dieser Versatz ist nicht durch Fehler bei der Thermostatierung oder bei der Auswertung zu erklären. Er ist reproduzierbar und lässt einen systematischen Fehler vermuten. Mit der Diskussion möglicher Ursachen für diesen Fehler beschäftigt sich Abschnitt 4.1.

Bei Ethylenglykol sind Streuung und Versatz so groß, dass auf die Verwendung der tieffrequenten Daten verzichtet wurde. Der damit einhergehende Informationsverlust wird als gering eingeschätzt, da der frequenznormierte Absorptionskoeffizient im Intervall von 400 bis $2 \mathrm{MHz}$ frequenzunabhängig ist und somit auch für den Bereich unter $2 \mathrm{MHz}$ keine größeren Veränderungen zu erwarten sind.

\subsection{Auswertung der Resonatordaten gegen unterschiedliche Referenzen}

Ein möglicher Grund für den Versatz zwischen den Datensätzen von hoch- und tieffrequenten Resonatoren könnte der erhebliche Unterschied im Schallabsorptionskoeffizienten von Polymerschmelzen und den Harnstoff-Wasser-Referenzlösungen sein: Solange die Flüssigkeitsdämpfung sehr hoch ist, wird der Anteil der apparativen Verluste an der gemessenen Halbwertsbreite gering sein (s. auch Abschnitt 3.2.1.2). Fehler, die bei der Bestimmung der apparativen Verluste durch unterschiedliche Eigenschaften von Referenz und Substanz verursacht werden, fallen dann dementsprechend wenig ins Gewicht. Bei kleinen Flüssigkeitsdämpfungen gewinnen sie jedoch an Bedeutung und könnten somit den zu kleinen Dämpfungen hin zunehmenden Versatz der Daten erklären.

Um diese Vermutung zu überprüfen, wurden die Resonatordaten der Oligoethylenglykolspektren gegen eine zweite Referenz ausgewertet, deren Dämpfungseigenschaften denen der Messprobe ähnlich sind. Dabei lag es nah, auf bereits 


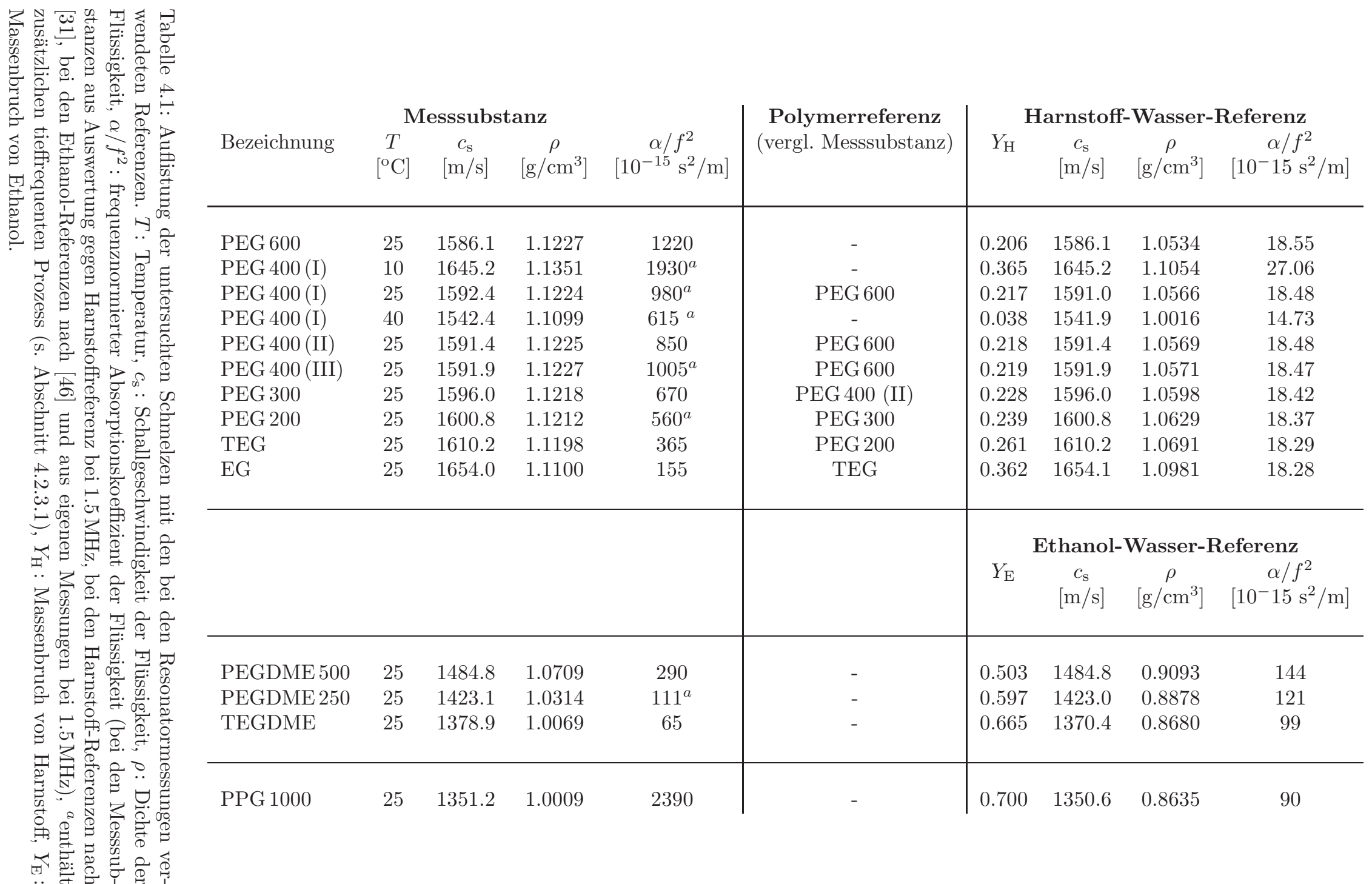


vorhandene Messergebnisse zurückzugreifen und das jeweils nächst längere PEGMolekül als Referenz zu wählen. Bei einem Unterschied im Molekulargewicht von $100 \mathrm{~g} / \mathrm{mol}$ reduziert sich dadurch der Unterschied in der Dämpfung beispielsweise bei PEG 300 von einem Faktor 34 gegenüber Harnstoff-Lösungen auf einen Faktor 0.8 gegenüber PEG 400 (II). Auch die Dichten der Ethylenglykololigomere untereinander stimmen besser überein als die von Polymer und Harnstoffreferenz (Tab. 4.1). Während jedoch die Harnstofflösung so angesetzt werden konnte, dass ihre Schallgeschwindigkeit genau der der Messsubstanz entspricht, musste bei den Polymerreferenzen ein Unterschied von maximal 3\% in Kauf genommen werden.

Ein grundsätzliches Problem des Referenzverfahrens ist, dass der Absorptionskoeffizient der Referenzlösung bereits bekannt sein muss. Deswegen musste zunächst ein Polymer gegen eine gut bekannte Harnstoffreferenz ausgewertet werden, um es anschließend als Ausgangsreferenz für die weiteren Auswertungsschritte verwenden zu können. Dafür wurde das Polymer mit der höchsten Dämpfung, PEG 600, ausgewählt, da hier nach den obigen Überlegungen mit den geringsten systematischen Fehlern aufgrund der unterschiedlichen Absorptionskoeffizienten zu rechnen ist. Ausgehend von diesem Datensatz wurde dann iterativ jeweils das nächst kürzere Oligomer bis hin zum Ethylenglykol ausgewertet. Mögliche Fehler, die bereits bei PEG 600 vorhanden waren oder durch eine wiederum nicht ganz ideale Referenz neu entstehen, können sich somit immer weiter fortpflanzen und systematisch vergrößern. Bei der späteren Gegenüberstellung der Ergebnisse beider Auswertungsverfahren ist deshalb zu berücksichtigen, dass durch den Wechsel der Referenz nicht mit einem geringeren Gesamtfehler zu rechnen ist, sondern dass vor allem verschiedene Fehlerquellen anders gewichtet werden: Genauere Übereinstimmung im Absorptionskoeffizienten wird erkauft mit größeren Ungenauigkeiten in der Schallgeschwindigkeit und mit einer Fehlerfortpflanzung aufgrund des iterativen Vorgehens. Dass dennoch ein Vergleich der Ergebnisse sinnvoll ist, zeigt Abbildung 4.3 am Beispiel von PEG 300:

Dargestellt sind die $\alpha / f^{2}$-Werte im Frequenzbereich der Resonatoren bei Auswertung der Messdaten gegen eine Harnstoff-Wasser-Referenz sowie gegen PEG 400 (II). Für die Auswertung gegen die Harnstoff-Lösung ist der bereits beschriebene Versatz der Datensätze von hoch- und tieffrequentem Resonator bei 1.5 MHz gut zu erkennen. Dabei fallen in beiden Datensätzen die $\alpha / f^{2}$-Werte mit zunehmender Frequenz merklich ab. Wertet man dagegen die Messdaten gegen eine Polymerreferenz aus, ist bei allen untersuchten Oligoethylenglykolen die gleiche Änderung des spektralen Verlaufs zu beobachten: Der Versatz zwischen den Datensätzen verschwindet praktisch vollständig. Ein Abwärtstrend der $\alpha / f^{2}$-Werte zu hohen Frequenzen des Resonatorbereichs ist nicht mehr zu erkennen, in einigen Fällen, wie auch bei PEG 300, zeigt sich eher eine entgegengesetzte Tendenz. Besonders im hochfrequenten Messbereich des 8-MHz-Resonators wachsen die $\alpha / f^{2}$-Werte jetzt mit der Frequenz leicht an, was einen schlechteren Übergang zu den Daten der tieffrequenten Pulszelle zur Folge hat (Abb. 4.4). Diese Abweichung ist allerdings besser zu verstehen als der Versatz zwischen den beiden Resonatoren, denn wegen der hohen Dämpfung der Polymerschmelzen müssen beim 8-MHz-Resonator schon 


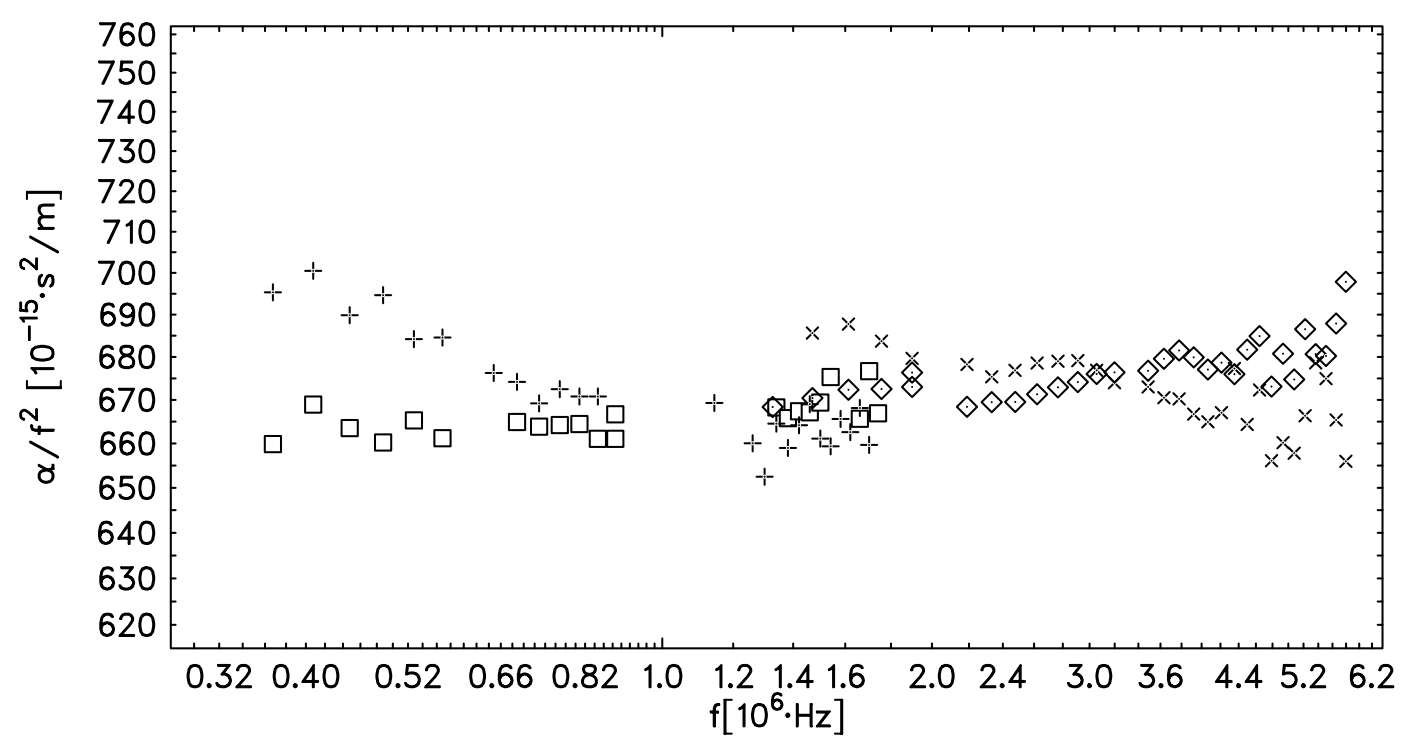

Abbildung 4.3: Frequenznormierter Absorptionskoeffizient von PEG 300, gemessen bei $25^{\circ} \mathrm{C}$ im Frequenzbereich von hoch- und tieffrequentem Resonator, ausgewertet gegen eine HarnstoffWasser-Referenzlösung (+: 1 MHz-Resonator, $\times$ : $8 \mathrm{MHz}-$ Resonator) und eine PEG 400 (II)Schmelze ( $\square$ : $1 \mathrm{MHz}$-Resonator, $\diamond: 8 \mathrm{MHz}$-Resonator).

bei vergleichsweise tiefen Frequenzen mehrere Nebenmoden mit angepasst werden, was erfahrungsgemäß zu einer eher zu groß bestimmten Halbwertsbreite führt. Der Anschluss von Resonator- und Pulszellendaten bei Auswertung gegen HarnstoffWasser-Referenz ist dagegen im Hinblick auf die schwierige Anpassungsprozedur erstaunlich gut. Auch wenn die Änderungen, die der Wechsel der Referenz mit sich bringt, bezogen auf das Gesamtspektrum klein sind, lässt der Vergleich den Schluss zu, dass es sich bei dem tieffrequenten Anstieg, der bei manchen Oligoethylenglykolen auftritt, nicht um einen weiteren Relaxationsprozess, sondern um ein Artefakt aufgrund einer nicht optimalen Referenz handelt.

Ein iteratives Auswertungsverfahren wie bei den PEG-Schmelzen wäre auch bei den Dimethylether-Derivaten denkbar. Aus folgenden Gründen wurde jedoch darauf verzichtet: Zum einen sind die Eigenschaften der drei untersuchten PEGDMESchmelzen bereits recht unterschiedlich (Tab. 4.1). Der Übergang von der EthanolWasser-Referenz zum nächst längeren gemessenen Oligomer des gleichen Typs lässt kaum bessere Ergebnisse erwarten. Zwar wären die Dichten ähnlicher, die Übereinstimmung in Schallgeschwindigkeit und Schallabsorption würde sich jedoch verschlechtern. Zum anderen weisen die PEGDME-Spektren allgemein größere Messungenauigkeiten auf. So war zusätzlich zu der höheren Streuung aufgrund der geringeren Flüssigkeitsdämpfung bei den Resonatormessungen eine erhöhte Drift der Resonanzfrequenz zu beobachten, die auch nach längeren Thermostatierzeiten nicht vollständig verschwand. Die mit dieser reproduzierbaren Instabilität einhergehenden systematischen Fehler in den gemessenen Halbwertsbreiten würden sich bei der iterativen Vorgehensweise des Auswertungsverfahrens noch weiter vergrößern. Eine solche Auswertung erscheint daher mit den vorhandenen Datensätzen 

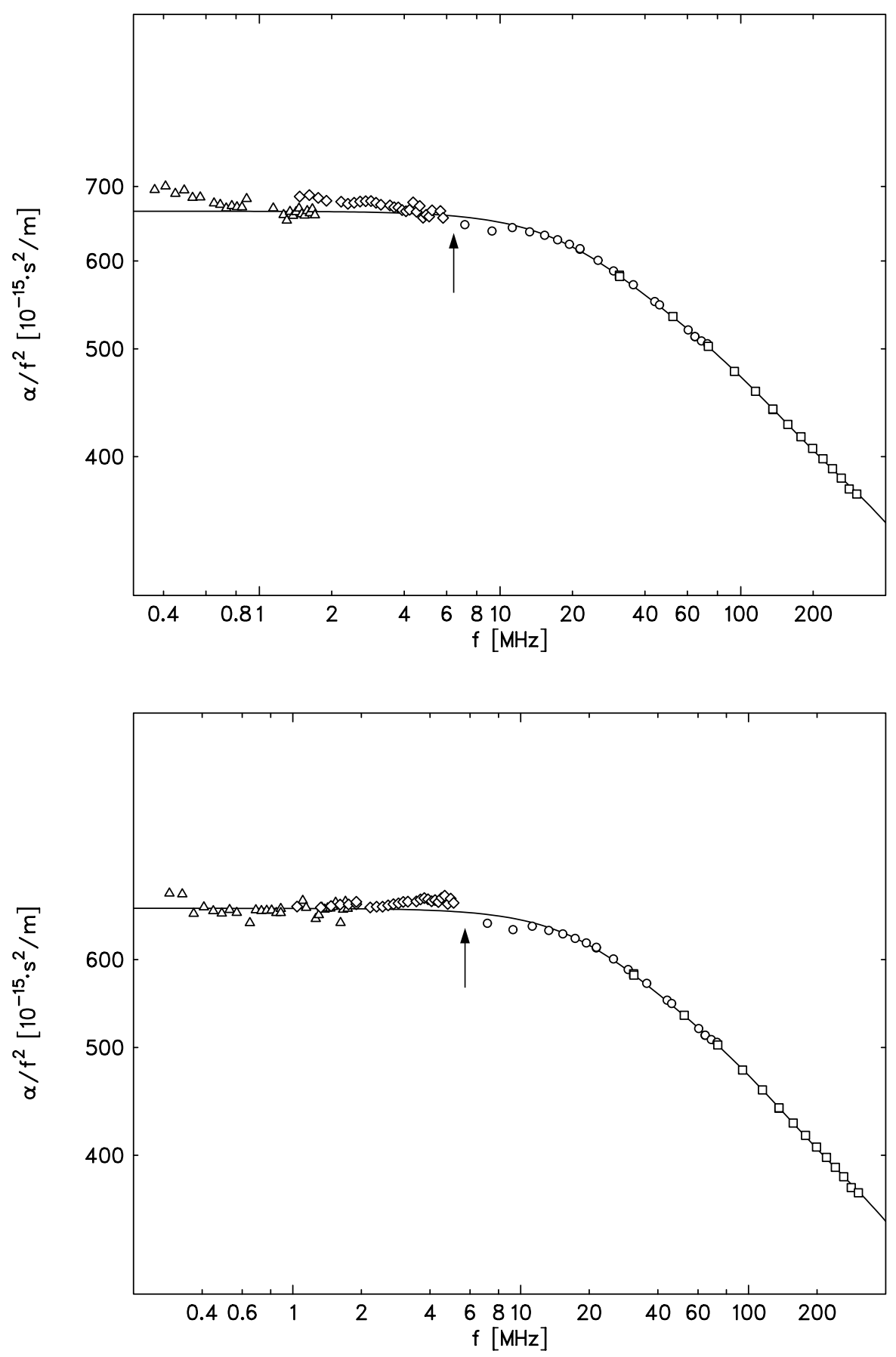

Abbildung 4.4: Teil des Absorptionsspektrums von PEG 300 bei $25^{\circ} \mathrm{C}$, Resonatordaten ausgewertet gegen Harnstoffreferenz (oben) und gegen PEG 400 (II) (unten). Der Pfeil markiert den Übergang von Resonatordaten zu den Daten der tieffrequenten Pulszelle. Verschiedene Symbole kennzeichnen verschiedene Messzellen. Als Orientierungshilfe ist das Ergebnis einer Anpassung mit drei Debye-Prozessen eingezeichnet (durchgezogene Linie). Die Daten der Hyperschallmesszelle wurden zugunsten eines besser aufgelösten tieffrequenten Bereichs nicht dargestellt. 
nicht sinnvoll.

Für eine möglichst einheitliche Diskussion der Ergebnisse werden im Folgenden immer die gegen Harnstoff-Wasser- bzw. Ethanol-Wasser-Referenz ausgewerteten Datensätze verwendet. Die Ergebnisse der Auswertung mit Polymerreferenz werden jedoch, soweit vorhanden, zur Abschätzung der Fehler mit herangezogen.

\subsection{Analytische Beschreibung der Oligoethylenglykolspektren}

\subsubsection{Anpassungsalgorithmus}

Zur analytischen Beschreibung der gemessenen Spektren $S\left(f_{n}\right)$ durch Spektralfunktionen $R\left(f_{n}, P_{1}, \ldots, P_{J}\right)$ wird mit Hilfe des Marquardt-Algorithmus [47] die Varianz

$$
\chi^{2}\left(P_{1}, \ldots, P_{J}\right)=\frac{1}{N-J-1} \sum_{n=1}^{N}\left(\frac{S\left(f_{n}\right)-R\left(f_{n}, P_{1}, \ldots, P_{J}\right)}{\Delta S\left(f_{n}\right)}\right)^{2}
$$

minimiert. Hierbei bezeichnen $f_{n}, n=1, \ldots, N$ die Messfrequenzen und $P_{1}, \ldots, P_{J}$ die Parameter der Spektralfunktion $R ; \Delta S\left(f_{n}\right)$ beschreibt den experimentellen Fehler von $S$ bei der Frequenz $f_{n}$ und führt zu einer Gewichtung der betrachteten Abweichung von Messwerten und Spektralfunktion in Gl. (4.1).

\subsubsection{Dispersion}

Da nicht für alle Spektralfunktionen ein geschlossener Ausdruck für die Dispersion der Schallgeschwindigkeit wie im Fall der Debye-Spektralfunktion (Gl. (3.7)) bekannt ist, wird die Dispersion nicht direkt als Parameter mitangepasst. Stattdessen wird sie berücksichtigt, indem die Daten zunächst durch eine Summe von Debye-Spektralfunktionen beschrieben werden, wobei der Ausdruck $\left(c_{\mathrm{s}}(\omega) / c_{s \infty}\right)$ in Gleichung (3.5) gleich eins gesetzt wird. Erst anschließend wird mit Hilfe von Gleichung (3.7) der Dispersionsverlauf berechnet und eine Korrektur der Messwerte mit dem entsprechenden Schallgeschwindigkeitsverhältnis vorgenommen. Durch erneute Anpassung der dispersionskorrigierten Messwerte mit der gewählten Spektralfunktion wird die Dispersion in die Funktionsparameter miteinbezogen. Bei den Oligoethylenglykolen ist ein Anwachsen der Dispersionsstufe mit zunehmender Kettenlänge zu beobachten: Für Ethylenglykol nimmt die Schallgeschwindigkeit im betrachteten Frequenzbereich um 1.6\% zu, bei PEG 300 erhöht sie sich um 5.6\%, bei PEG 600 um 5.9\%. Das längste im Rahmen dieser Arbeit untersuchte Polymer, PPG 1000, zeigt einen Anstieg von $1351 \mathrm{~m} / \mathrm{s}$ im Resonator- auf 1493 m/s im Hyperschallbereich, was einer Änderung von $10.5 \%$ entspricht. Trotz dieser teilweise deutlichen Zunahme der Schallgeschwindigkeit ändern sich die Anpassungsparameter durch die Dispersionskorrektur nur wenig. Am meisten macht 


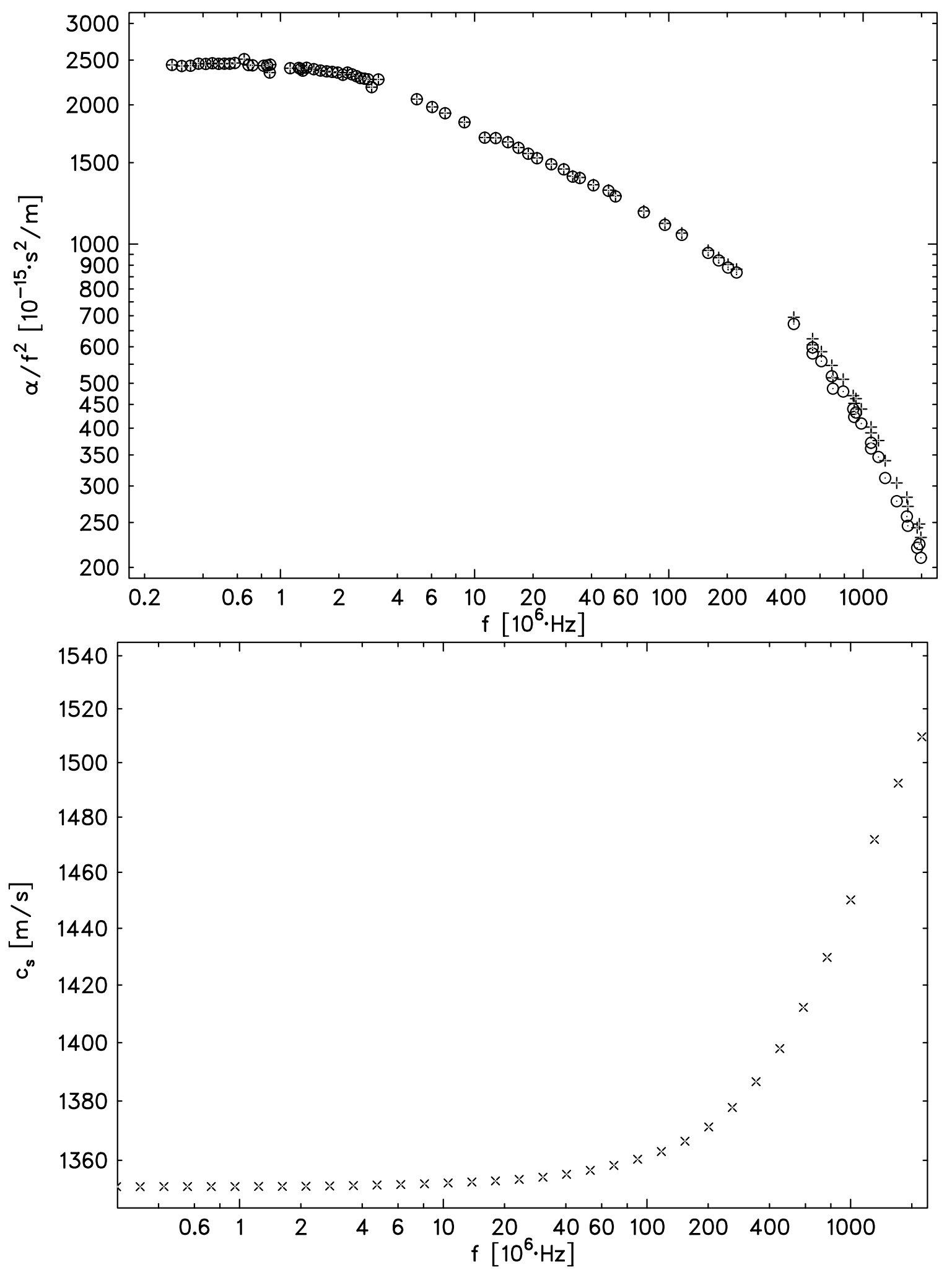

Abbildung 4.5: Oben: Messwerte von PPG 1000 bei $25^{\circ} \mathrm{C}$ vor $(\odot)$ und nach $(+)$ der Dispersionkorrektur. Unten: Berechnete Dispersion der Schallgeschwindigkeit für PPG 1000 bei $25^{\circ} \mathrm{C}$ im untersuchten Frequenzbereich. 


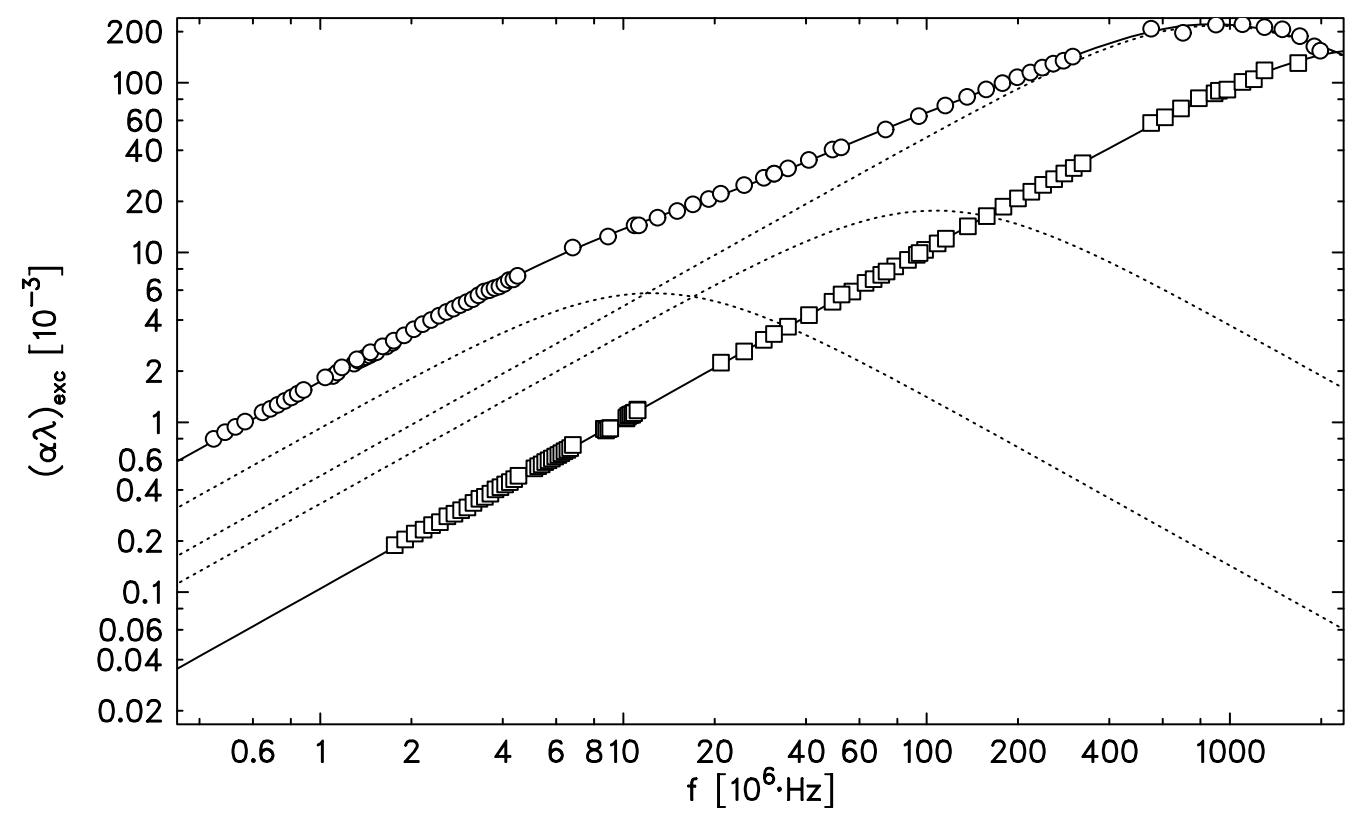

Abbildung 4.6: Exzessdämpfung von PEG600 (०) und EG ( $\square$ ) bei $25^{\circ} \mathrm{C}$ als Funktion der Frequenz. Durchgezogene Linien: Ergebnis der Anpassung mit Debye-Spektralfunktionen, gepunktete Linien: Darstellung der drei einzelnen Debye-Spektralterme aus der Anpassung von PEG 600.

sich die Dispersion im hochfrequenten Bereich des Spektrums bemerkbar. Hier sind die Anpassungsparameter jedoch wegen des schwer zu bestimmenden Hintergrundanteils ohnehin mit so großen Fehlern behaftet, dass Parameteränderungen durch die Dispersionskorrektur meist schon im Rahmen der Anpassungsfehler liegen.

Da die Dispersionskorrektur keine für die Interpretation der Spektren maßgeblichen Änderungen mit sich bringt, werden im Folgenden immer die aus den Anpassrechnungen gewonnenen Parameterwerte der unkorrigierten Datensätze betrachtet. Dabei beinhalten die angegebenen Fehler aber immer den Fehler der Anpassung und den Fehler, der durch den Verzicht auf die Dispersionskorrektur verursacht wird.

\subsubsection{Beschreibung durch Debye-Spektralfunktionen}

Die Absorptionsspektren der Oligoethylenglykole lassen sich sehr gut durch eine Summe von Debye-Spektralfunktionen beschreiben. Das gilt auch für die untersuchten Dimethyletherderivate und für die PPG-Schmelze. Abbildung 4.6 zeigt die auf diese Weise angepassten Spektren für Ethylenglykol und ein Oligoethylenglykol bei $25^{\circ} \mathrm{C}$. Dargestellt ist die Exzessabsorption

$$
(\alpha \lambda)_{\mathrm{exc}}=(\alpha \lambda)-B f
$$

als Funktion der Frequenz.

In Tabelle 4.2 sind die Parameter aufgelistet, wie man sie aus der Anpassung der nicht dispersionskorrigierten, gegen Harnstoff- bzw. Ethanolreferenz ausgewerteten Datensätze erhält (vergl. Abschnitte 4.1, 4.2.2). Die Parameter, die sich aus 


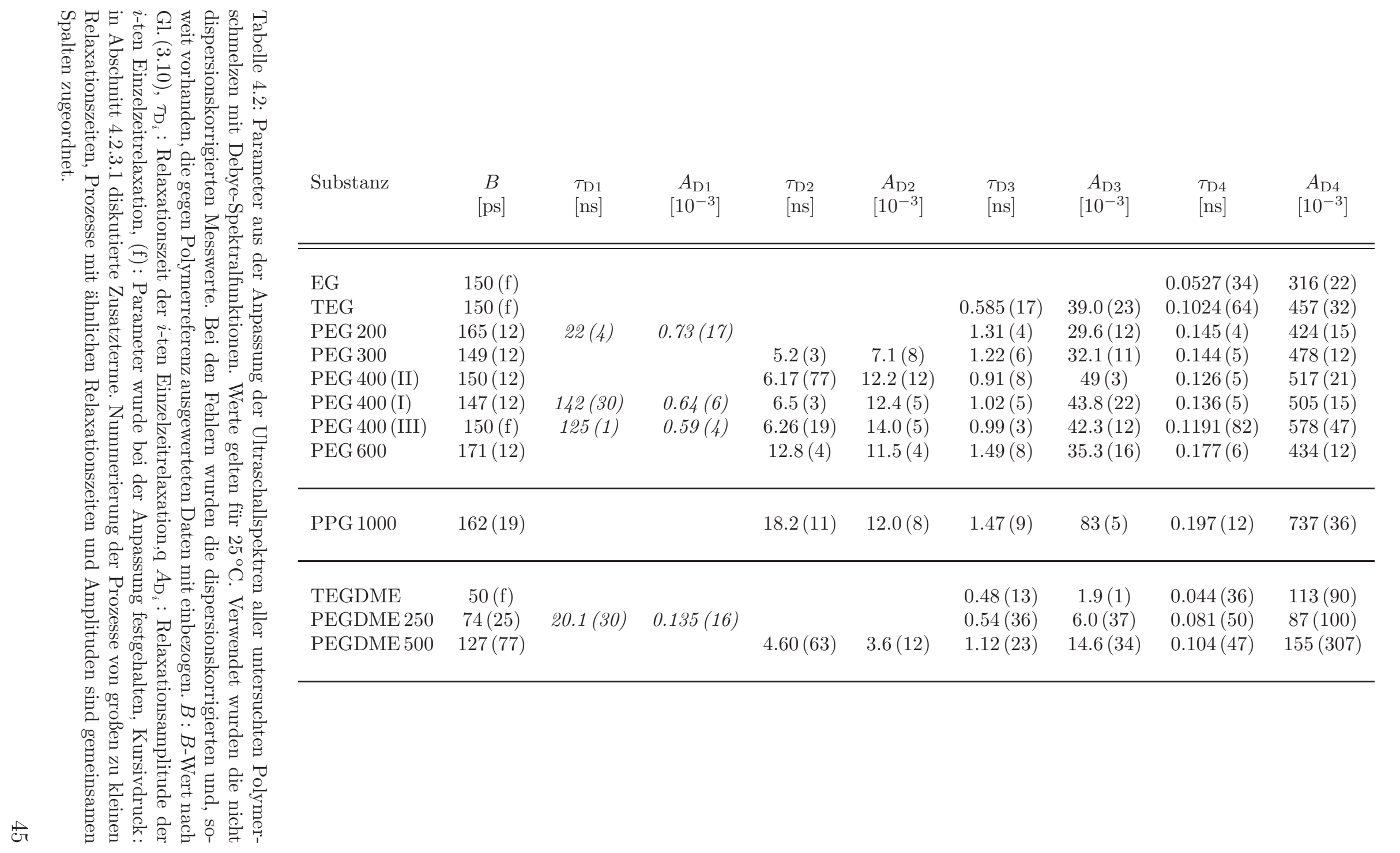


der Anpassung der dispersionskorrigierten bzw. gegen Polymerreferenz ausgewerteten Daten ergeben haben, liegen innerhalb der angegebenen Anpassungsfehler. Wo mangels geeigneter Referenz keine Ergebnisse für die Auswertung mit einer Polymerreferenz vorliegen, wurden nur die dispersionskorrigierten Datensätze in die Fehlerabschätzung einbezogen.

Bei den kurzkettigen Schmelzen (EG/TEG/TEGDME) musste der der $B$-Wert als Parameter festgehalten werden, um eine stabile Anpassung zu bekommen. Der auf Grund des begrenzten Messfrequenzbereichs schlecht zu bestimmenden Hintergrundsanteil erlaubt hier große Variationsmöglichkeiten, so dass dieser Wert und damit auch die Parameter der hochfrequenten Einzelzeitrelaxation besonders fehlerbehaftet sind. Die angegebenen Parameter fügen sich folgerichtig in das Gesamtbild aus allen Spektren ein.

Die Anzahl der zur Beschreibung der Spektren benötigten Debye-Funktionen wird zu kürzeren Kettenlängen hin kleiner. Während bei PEG 200, 300, 400 (II) und 600 drei Debye-Spektralfunktionen notwendig sind, um die Spektren ausreichend wiedergeben zu können, sind zur Beschreibung der Spektren von TEG nur noch zwei Debye-Funktionen, für das Monomer EG schließlich nur noch eine einzige notwendig. Diese Tendenz findet sich bei den anderen untersuchten Polymerschmelzen ebenfalls wieder.

\subsubsection{Zusatzterme bei PEG 200, PEG 400 (I/III) und PEGDME 250}

Eine Besonderheit stellen die mit (I) und (III) bezeichneten PEG 400-Schmelzen dar, die aus unterschiedlichen Produktionsreihen des gleichen Herstellers stammen. Diese Chargen weisen im Gegensatz zu PEG 400 (II) einen charakteristischen tieffrequenten Anstieg der $\alpha / f^{2}$-Werte auf, der durch einen zusätzlichen DebyeSpektralterm erfasst werden kann (Abb.4.7). Wie ein Vergleich mit den Anpassungsparametern von PEG 400 (II) zeigt, verhält sich dieser Prozess additiv, beeinflusst also die anderen molekularen Vorgänge nicht. Eine mögliche Ursache für sein Auftreten könnten bereits bei der Herstellung verursachte Verunreinigungen sein. Dafür spricht, dass beim Öffnen mehrerer originalverpackter Proben der Charge (I) sichtbare Verunreinigungen festgestellt wurden. Für spätere Messungen wurde daher möglichst auf die Charge II zurückgegriffen. Ein Zusatzterm mit ähnlicher Relaxationszeit und -amplitude wie bei dem zuvor beschriebenen tieffrequenten Term wurde auch bei den untersuchten Chargen von PEG 200 und PEGDME 250 beobachtet. Sein im Hinblick auf die Kettenlänge unregelmäßiges Auftreten legt auch hier die Vermutung nahe, dass es sich um einen durch Verunreinigungen verursachten Absorptionsprozess handelt. Abgesehen von diesem zusätzlichen Term fügen sich die Parameter beider Spektren sehr gut in das später dargestellte Gesamtbild ein. Er wird daher wie der tieffrequente Prozess von PEG 400 (I/III) als additiver Zusatzeffekt behandelt und bei der folgenden Diskussion der Parameterverläufe nicht mitberücksichtigt. Die Funktionsparameter der Zusatzterme sind in Tab.4.2 durch Kursivdruck kenntlich gemacht. 


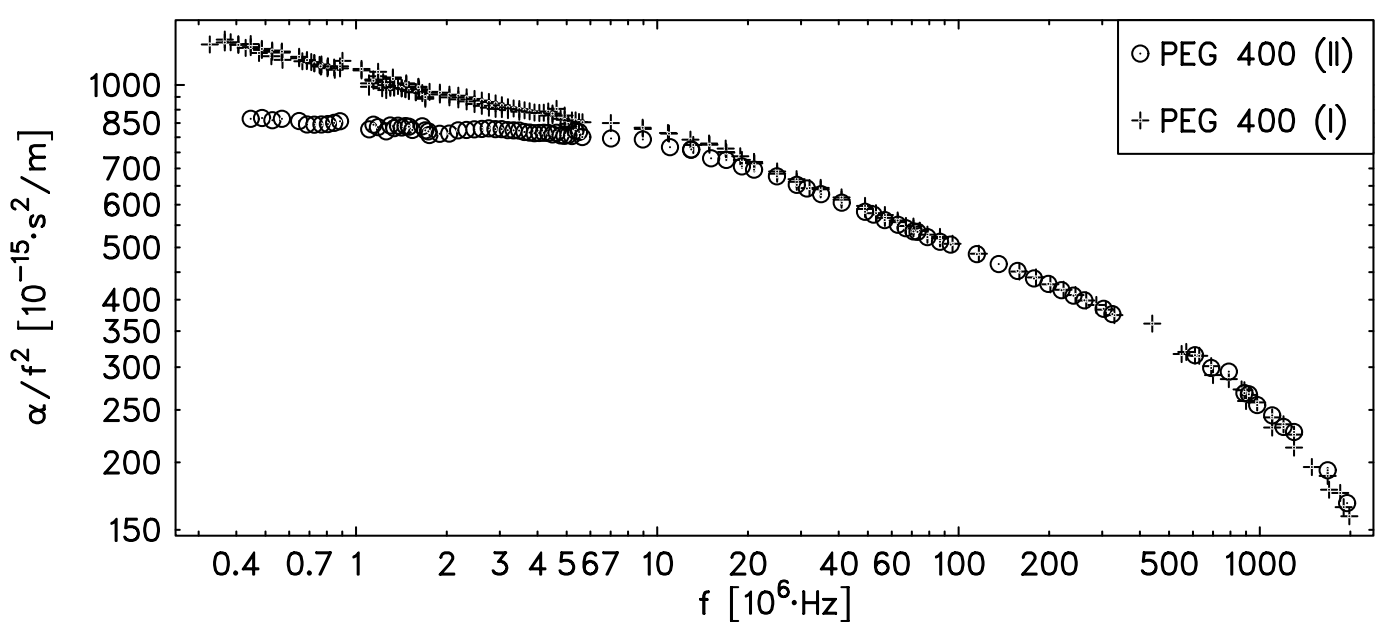

Abbildung 4.7: Vergleich der Absorptionsspektren von PEG 400 (I) und (II), gemessen bei $25^{\circ} \mathrm{C}$.

\subsubsection{Beschreibung durch andere Spektralfunktionen}

Beschreibungen der Oligoethylenglykolspektren durch andere Spektralfunktionen sind zwar möglich, sie führen aber nicht zu einem vergleichbar konsistenten Verlauf der Anpassungsparameter wie die Beschreibung mit Debye-Spektralfunktionen. Erwähnenswert sind die Ergebnisse des Versuchs, die Spektren durch Cole-ColeRelaxationsfunktionen zu beschreiben, denen eine kontinuierliche Relaxationszeitverteilung zugrunde liegt (vergl. Abschnitt 5.1.1). Diese Art, die Spektren darzustellen, liegt aus zwei Gründen nahe: Zum einen besitzen nur die sehr kurzen Oligomere EG, DEG und TEG eine wohldefinierte Kettenlänge, die Kettenlängen aller anderen polymeren Substanzen unterliegen einer (schmalen) Verteilung, die sich als Relaxationszeitverteilung in den Spektren widerspiegeln könnte. Zum anderen lassen sich dielektrische Spektren von Oligoethylenglykolen gut durch eine Cole-Cole-Relaxationsfunktion beschreiben (Abschnitt 6.1), während die Verwendung einer Debye-Funktion nicht ausreicht.

Die Beschreibung der Ultraschallspektren durch Cole-Cole-Funktionen liefert jedoch keine neuen Ergebnisse, sondern führt wieder auf die Debye-Spektralfunktionen zurück: Die Breite der Relaxationszeitverteilungen, die die Regressionsanalyse bestimmt, ist in den meisten Fällen so klein, dass im Rahmen der Anpassungsfehler die Cole-Cole-Funktion einer Debye-Spektralfunktion entspricht. Um eine Überbestimmung der Spektren durch zu viele freie Parameter zu vermeiden, wurde daher die Debye-Darstellung bevorzugt.

\subsection{Diskussion der Ergebnisse}

Die untersuchten linearen Oligomerketten können in einer Vielzahl von Konformationen vorliegen, die durch Rotation um die Kohlenstoff-Kohlenstoff- oder Kohlenstoff-Sauerstoff-Bindungen auseinander hervorgehen. Zwischen diesen Konforma- 


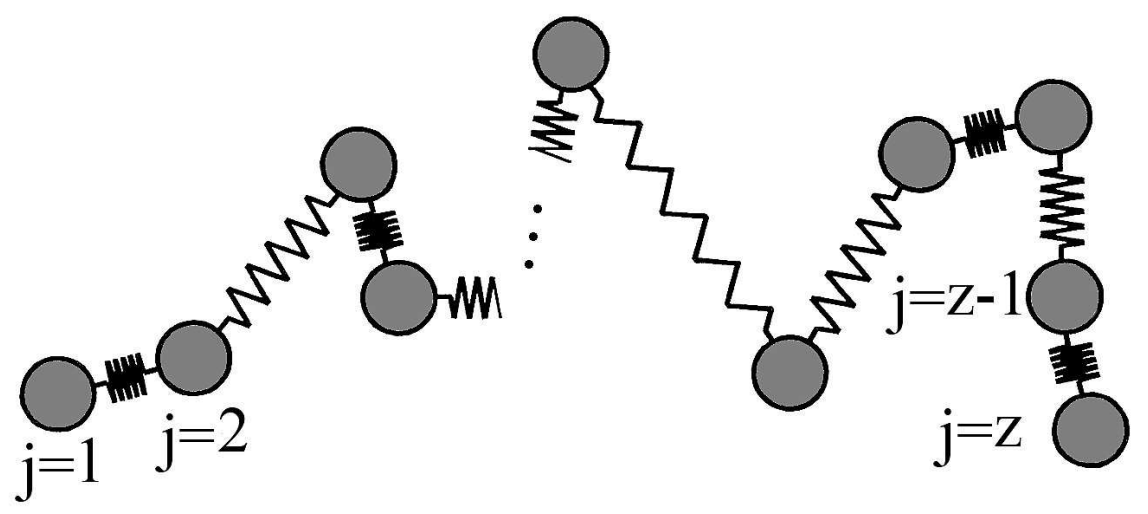

Abbildung 4.8: Rouse-Kette zusammengesetzt aus z Massen, verbunden durch Hookesche Federn. Nach [48].

tionen finden ständig thermisch angeregte Übergänge statt. Häufig ist der Übergang zwischen derartigen Rotationsisomeren mit einem veränderten Eigenvolumen verbunden und koppelt daher an Dichteschwankungen in einem Schallfeld. Erfahrungsgemäß relaxieren viele solcher Übergänge im betrachteten UltraschallFrequenzbereich. Es ist somit anzunehmen, dass die in den Ultraschallspektren beobachteten Absorptionsprozesse auf Konformationsänderungen der Oligomerketten beruhen. Darauf deuten auch die zu kürzeren Ketten hin abnehmenden tieffrequenten $\alpha / f^{2}$-Werte und die abnehmende Anzahl der für die Beschreibung der Spektren benötigten Debye-Spektralfunktionen hin.

\subsubsection{Rouse-Modell und Modell gedämpfter Torsionsoszillatoren}

Eines der grundlegendsten Modelle zur Beschreibung der Kettendynamik von Polymeren ist das Modell von Rouse [6], das von Tobolsky und Aklonis um die Einbeziehung von Torsionsbewegungen erweitert wurde [8]. Auf der Grundlage dieser vergleichsweise einfachen Modelle soll versucht werden, die Spektren der untersuchten Polymerschmelzen zu verstehen. Sie werden in diesem Abschnitt vorgestellt.

\subsubsection{Rouse-Modell}

Rouse [6] zerlegt die Polymerkette in Segmente, die so groß sind, dass der Endzu-End-Abstand jedes Segments durch eine Gauß-Verteilung beschrieben werden kann (Gaußsche Kette). Hierfür sind zwei Voraussetzungen notwendig:

Zum einen muss die Segmentlänge deutlich größer als die Persistenzlänge, d.h. die Zerfallslänge der Orientierungskorrelation, gewählt werden. Zum anderen darf keine Volumenausschlusswechselwirkung stattfinden. Diese Wechselwirkung berücksichtigt den Volumenbedarf der Kettensegmente. Sie verbietet, dass sich zwei Segmente durchdringen oder am gleichen Ort aufhalten und kann durch eine kurzreichweitige Abstoßung beschrieben werden. Im Vergleich zur Gaußschen Kette 
würde sie zu einer größeren Ausdehnung des Polymers führen.

Effektiv verschwindet die Volumenausschlusswechselwirkung jedoch in zwei Fällen: In einer so genannten $\theta$-Lösung, in der die Abstoßung zwischen zwei Kettensegmenten gerade durch die Abstoßung zwischen Polymer und (schlechtem) Lösungsmittel kompensiert wird, oder in einer Polymerschmelze, bei der die Abstoßung zwischen zwei Segmenten einer Kette und denen verschiedener Ketten gleich groß ist.

Da die End-zu-End-Abstände der Segmente einer Gaußverteilung unterliegen, können ihre Endpunkte als durch Hookesche Federn verbunden modelliert werden. Ihre Federkonstante $D$ hängt über die Gleichung $D=3 k T / \sigma^{2}$ mit der absoluten Temperatur $T$ und dem mittleren quadratischen End-zu-End-Abstand $\sigma^{2}$ zusammen und weist damit die für eine entropische Feder charakteristische Temperaturabhängigkeit auf.

Die Polymerkette wird nun im Rouse-Modell als ein gedämpftes Feder-MasseSystem aus $z$ Massen und $(z-1)$ Federn, den Segmenten, aufgefasst (Abb. 4.8). Für ein Homopolymer werden dabei die Segmente gleich groß gewählt. Für die einzelnen Massen lassen sich dann folgenden Bewegungsgleichungen aufstellen:

$$
\begin{aligned}
& \zeta \dot{x}_{j}(t)=D \cdot\left(x_{j+1}(t)-x_{j}(t)\right)+D \cdot\left(x_{j-1}(t)-x_{j}(t)\right), \quad j=2, \ldots, z-1 \\
& \zeta \dot{x}_{1}(t)=D \cdot\left(x_{2}(t)-x_{1}(t)\right) \\
& \zeta \dot{x}_{z}(t)=D \cdot\left(x_{z-1}(t)-x_{z}(t)\right)
\end{aligned}
$$

wobei $x_{j}(t)$ die Verschiebung der $j$-ten Masse aus ihrer Gleichgewichtsposition zum Zeitpunkt $t$ bezeichnet. Die Dämpfungskonstante $\zeta$ berücksichtigt die viskose Reibung der Massen in der umgebenden Flüssigkeit.

Unter der Annahme, dass es sich bei dem Feder-Masse-System um ein überdämpftes System handelt, sind die Trägheitsterme $m \ddot{x}_{j}(t)$ in den Bewegungsgleichungen gegenüber den Reibungstermen vernachlässigt worden. Die Bewegungsgleichungen sind für alle drei Raumrichtungen gleich und ungekoppelt. Daher genügt es, stellvertretend die $x$-Richtung zu betrachten.

Das Differentialgleichungssystem besitzt z zeitabhängige Lösungen, die Eigenmoden

$$
\boldsymbol{x}^{p}(t)=\boldsymbol{x}_{0}^{p} \cdot e^{-t / \tau_{p}},
$$

mit $\boldsymbol{x}_{0}^{p}=\left(x_{1}^{p}, \ldots, x_{z}^{p}\right)$ und $x_{j}^{p}=\cos \left(\frac{p \pi}{z}\left(j-\frac{1}{2}\right)\right), j=1, \ldots, z$. Sie werden durch die Relaxationszeiten

$$
\tau_{p}=\left(\frac{4 D}{\zeta} \sin ^{2}\left(\frac{p \pi}{2 z}\right)\right)^{-1}
$$

charakterisiert, wobei $p=0, \ldots, z-1$ die Nummer der Moden bezeichnet. Die nullte Mode $(p=0)$ entspricht einer reinen Translationsbewegung der Kette und ist daher für spätere Überlegungen nicht relevant.

In den Abbildungen 4.9, 4.10 und 4.11 wird die Bewegung der Polymerkette in einer Dimension für die ersten drei Moden veranschaulicht. Dargestellt ist die Relaxation der zwölf Massen einer Kette mit elf Segmenten nach einer einmaligen 


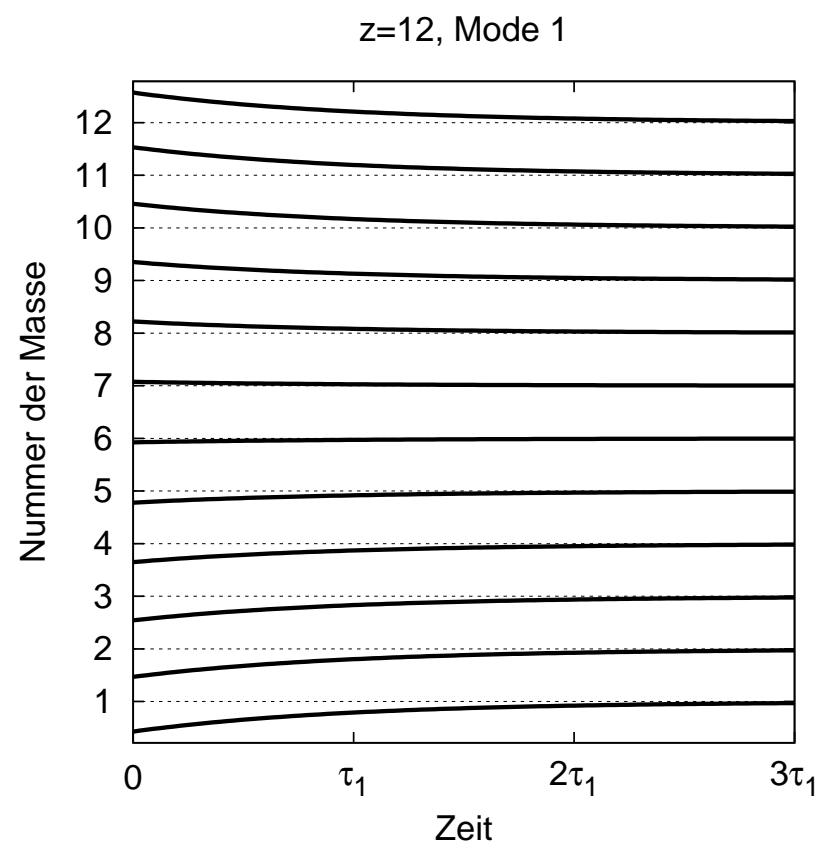

Abbildung 4.9: Veranschaulichung der ersten Rouse-Mode $(p=1)$ für eine Kette mit 12 Massen. Durchgezogenen Linien: Auslenkung der einzelnen Massen als Funktion der Zeit. Gestrichelte Linien markieren die Gleichgewichtspositionen.

Störung zum Zeitpunkt $t=0$ zurück in ihre Gleichgewichtsposition. In der Darstellung ist vereinfachend angenommen, dass die Massen im Ruhezustand gleiche Abstände haben. Die erste Mode entspricht dann einer gleichzeitigen Expansion bzw. Kompression aller Federn, die Gesamtlänge des Kette ist gegenüber der Gleichgewichtslage erhöht bzw. vermindert. Bei der zweiten Mode tritt eine Zweiteilung in der Bewegung der Massen auf: Während sich der eine Teil der Kette zusammenzieht, dehnt sich der andere Teil aus. Entsprechend kommt es bei der dritten Mode zu einer Dreiteilung der Kette usw.

Für ausreichend lange Ketten und Moden niedriger Ordnung, d.h. für kleines $(p / z)$, kann der Sinus in Gleichung (4.6) durch sein Argument genähert werden. Dies führt auf die einfache Beziehung

$$
\tau_{p}=\frac{\zeta}{D \pi^{2}} \cdot \frac{z^{2}}{p^{2}}=\tau_{1} \cdot \frac{1}{p^{2}}, \quad p=1, \ldots, z-1 .
$$

In dieser Näherung wächst also zum einen die Relaxationszeit der $p$-ten Mode quadratisch mit der Anzahl der Massen an, zum anderen hängen Relaxationszeiten höherer Moden nur von der größten Relaxationszeit $\tau_{1}$ und der Ordnung $p$ der Mode ab.

\subsubsection{Modell gedämpfter Torsionsoszillatoren (DTO-Modell)}

Das Rouse-Modell wurde von Tobolsky und Aklonis [8] um die Beschreibung von Torsionsbewegungen ergänzt. An die Stelle der Rouseschen Segmente mit gaußver- 


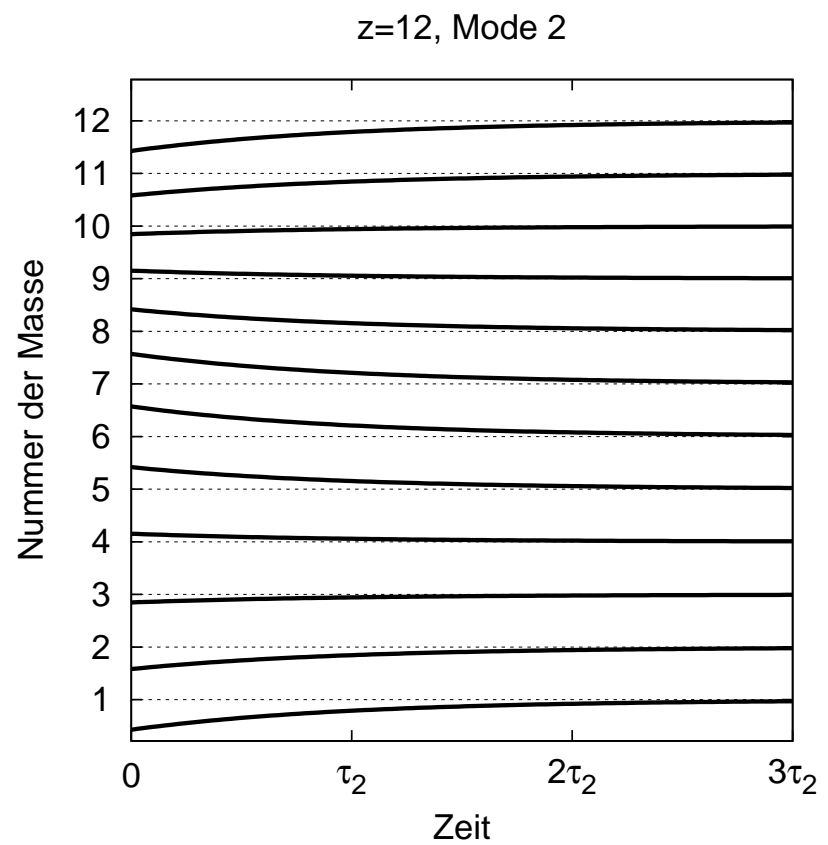

Abbildung 4.10: Veranschaulichung der zweiten Rouse-Mode $(p=2)$ für eine Kette mit 12 Massen. Für Erläuterungen siehe 4.9.

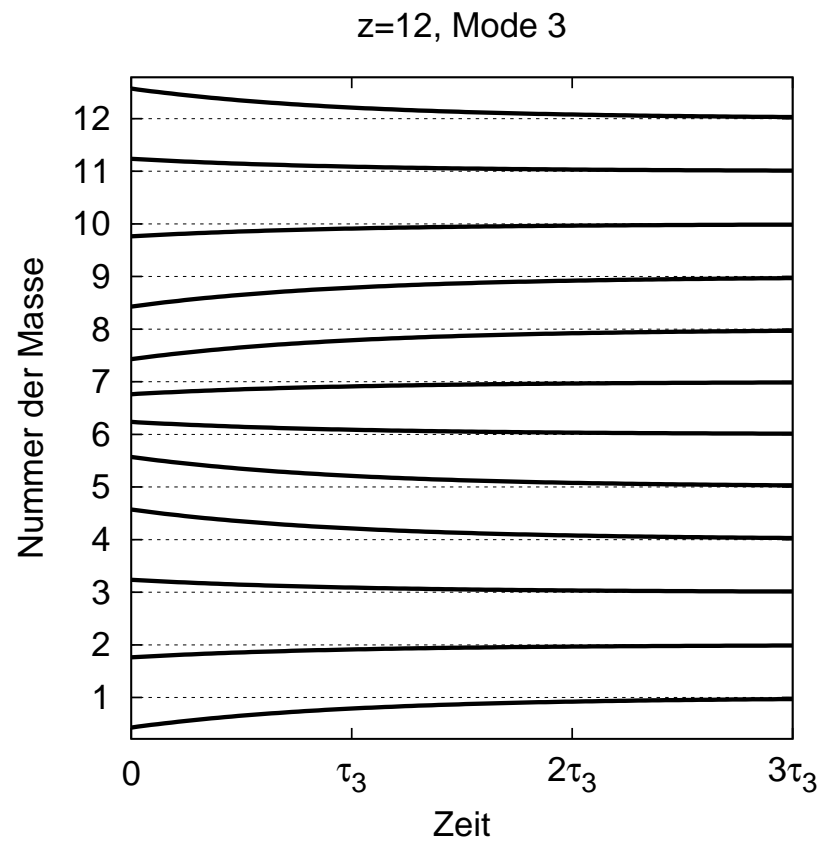

Abbildung 4.11: Veranschaulichung der dritten Rouse-Mode $(p=3)$ für eine Kette mit 12 Massen. Für Erläuterungen siehe 4.9 . 
teilten End-zu-End-Abständen treten Kettenabschnitte mit unabhängigen Drehwinkeln um die Molekülachse.

Die Torsionswinkel im DTO-Modell folgen denselben Gleichungen wie die Positionen der Massen im Rouse-Modell, wobei die mit der Translationsbewegung und den Eigenschaften des Rouse-Segments verknüpften physikalischen Parameter durch die entsprechenden Größen der Torsionsbewegung und des Torsionssegments zu ersetzen sind. Da die Korrelation der Torsionswinkel längs der Kontur schneller abnimmt als die der Position der Atome, können diese Segmente wesentlich kürzer sein als die Rouse-Segmente. Tobolsky und Aklonis geben eine Länge von zwei bis drei rotationsfähigen (C-C-)Bindungen an, während sich die Rouse-Segmente über etwa zehn Bindungen erstrecken [7], was etwa dem Dreifachen der Persistenzlänge von n-Alkanen entspricht [49].

Für Reibungs- und Federkonstante im DTO-Modell geben Tobolsky und DuPré [7] eine Interpretation an, die auf dem klassischen Modell der Einzelzeitrelaxation beruht [50]: Sie gehen davon aus, dass jeder Torsionsoszillator zwei diskrete Gleichgewichtspositionen besitzt, die durch eine Energiebarriere der Höhe $\Delta H$ voneinander getrennt sind. Um jede dieser Gleichgewichtspositionen schwingt der Oszillator mit einer charakteristischen Torsionsfrequenz $f_{\mathrm{t}}$, die von der Masse $m$ des Oszillators und der durch eine Torsionsfederhärte $D_{\mathrm{t}}$ beschriebenen Steilheit des Potentials um die Gleichgewichtsposition abhängt. Durch thermische Stöße kann ein Oszillator zwischen den Gleichgewichtszuständen hin- und herwechseln. Dabei ist die Übergangsrate desto größer, je kleiner $\Delta H$ und je größer die Anlauffrequenz $f_{\mathrm{t}}$ gegen den Potentialberg ist. Durch den Vergleich der inversen Übergangsrate mit der kleinsten Relaxationszeit im Rouse-Modell finden Tobolsky und DuPré die folgende Abschätzung für die Dämpfungskonstante [7]:

$$
\zeta_{\mathrm{t}}=8 \pi^{2} m f_{\mathrm{t}} e^{\Delta H / k T}
$$

Hierbei bezeichnen $k$ und $T$ die Boltzmannkonstante bzw. die absolute Temperatur. Die Dämpfung der Torsionsbewegung wird also nicht wie im Rouse-Modell auf die viskose Reibung eines Segments in der umgebenen Flüssigkeit, sondern auf die innermolekulare Behinderung der Rotation eines Oszillators zurückgeführt.

\subsubsection{Beschreibung der Spektren auf Grundlage des Rouse-/DTO-Modells}

Dieser Abschnitt beschäftigt sich mit der Frage, ob die Ultraschallspektren der Oligomerschmelzen mit Hilfe des Rouse- oder des DTO-Modells verstanden werden können. Da sich die Modelle nur in der absoluten Größe der Parameter (Federkonstante etc.) unterscheiden, sind ihre Vorhersagen, beispielsweise für die Relaxationszeiten, bis auf eine Skalierung gleich. Daher werden beide Ansätze im Folgenden zunächst nicht unterschieden und gemeinsam unter dem Namen „Rouse-Modell“ zusammengefasst. 
Eine der Grundaussagen des Rouse-Modells ist, dass die komplexe Kettenbewegung als Überlagerung von Eigenmoden mit diskreten, gekoppelten Relaxationszeiten aufgefasst werden kann. Übertragen auf die Ultraschallspektren sind die beobachteten Einzelzeitrelaxationen dann entsprechend als Eigenmoden der Oligomerkettendynamik anzusehen. Dabei sollte mit zunehmender Kettenlänge sowohl die Anzahl der auftretenden Moden als auch die Relaxationszeit der Grundmode $\tau_{1}$ anwachsen. Umgekehrt ist zu erwarten, dass bei ausreichend kurzen Ketten bestimmte (höhere) Moden nicht mehr auftreten können, da sie eine Unterteilung der Kette in Segmente, deren Länge im Bereich oder unterhalb der Persistenzlänge liegt, erfordern würden. Diese Segmente könnten aber keine von den Nachbarsegmenten unabhängige Bewegung mehr ausführen.

Beginnt man mit der Zuordnung der Moden bei Ethylenglykol, so kann es sich bei der Einzelzeitrelaxation mit der in Tabelle 4.2 als $\tau_{\mathrm{D} 4}$ bezeichneten Relaxationszeit nur um die Grundmode $(p=1)$ handeln. Offenbar ist die monomere Einheit, bestehend aus nur einer C-C- und zwei C-O-Bindungen, noch zu kurz, um die Anregung höherer Moden zu ermöglichen. Eine weitere Mode tritt bei Triethylenglykol (mittlere Anzahl monomerer Einheiten $\bar{n}=3$ ) auf. Die Grundmode verschiebt sich dabei wegen der höheren Kettenlänge wie erwartet zu tieferen Frequenzen, was sich in der Erhöhung der Relaxationszeit von $0.0527 \mathrm{~ns}$ auf $0.585 \mathrm{~ns}$ zeigt (Parameter von Mode 1 jetzt in Spalte $\tau_{\mathrm{D} 3}$ bzw. $A_{\mathrm{D} 3}$, nächst Höhere in Spalte $\tau_{\mathrm{D} 4}$ bzw. $\left.A_{\mathrm{D} 4}\right)$. Im Fall von PEG 200 wachsen zwar die Relaxationszeiten der Moden weiter an, es treten jedoch weiterhin nur zwei Moden auf ${ }^{2}$. Erst PEG $300(\bar{n}=6.4)$ erlaubt offenbar die Anregung einer noch höheren Mode. Wieder verschieben sich dabei die beiden tieferen Moden zu kleineren Frequenzen. Nach diesem Ansatz entspricht also immer die tieffrequenteste Einzelzeitrelaxation der Grundmode. Höhere Moden, deren genaue Ordnung noch zu ermitteln ist, schließen sich zu größeren Frequenzen an. Dabei wird davon ausgegangen, dass Moden, die bei kurzen Ketten auftreten, auch bei längeren Oligomerketten angeregt werden können.

Auf den ersten Blick scheint dieser Interpretationsansatz jedoch bei höheren Kettenlängen nicht zu passen: Obwohl sich die Kettenlänge von PEG 300 zu PEG 600 verdoppelt, treten keine weiteren Einzelzeitrelaxationen auf. Auch die Spektren von PEGDME 500 ( $\bar{n}=10.3)$ und des noch einmal längeren PPG $1000(\bar{n}=16.9)$ können bereits durch drei Debye-Spektralfunktionen beschrieben werden. Es ist allerdings durchaus denkbar, dass die 3-Debye-Darstellung der Spektren von PEG 400, PEG 600, PPG 1000 und PEGDME 500 zwar die Messwerte ausreichend gut wiedergibt, den Spektren aber weitere Einzelzeitrelaxationen zugrunde liegen, die lediglich so dicht aufeinanderfolgen, dass sie nicht mehr einzeln aufgelöst werden können. Hierfür spricht auch der Modenabstand, der vom Rouse-Modell vorhergesagt wird: Da die Relaxationszeiten $\tau_{p}$ mit $p^{-2}$ kleiner werden, nimmt der Abstand zweier benachbarter Einzelzeitrelaxationen in der logarithmischen Auftragung mit dem Faktor zwischen den Relaxationsfrequenzen $f_{p}=1 /\left(2 \pi \tau_{p}\right)$ immer mehr ab: $f_{2} / f_{1}=4, f_{3} / f_{2}=9 / 4, f_{4} / f_{3}=16 / 9$ usw. Erfahrungsgemäß können aber nur Ein-

\footnotetext{
${ }^{2}$ Zusatzeffekte nach Abschnitt 4.2 .3 .1 werden nicht berücksichtigt.
} 
zelzeitrelaxationen vom Auge getrennt werden, deren Relaxationsfrequenzen selbst bei gleichen Amplituden wenigstens um einen Faktor 7 auseinanderliegen.

\subsubsection{Abhängigkeit der Relaxationszeit von der Kettenlänge}

Bevor der Versuch unternommen wird, die Spektren mit mehr als drei Einzelzeitrelaxationen zu beschreiben, sollte zunächst überprüft werden, wie weit für die obige Modenzuordnung überhaupt der von Rouse vorhergesagte Zusammenhang zwischen Relaxationszeit und Kettenlänge erfüllt ist. Für die kurzen Ketten wäre dabei eigentlich die exakte Gleichung (4.6) der Näherungsgleichung (4.7) vorzuziehen und als Überprüfungsgrundlage zu wählen. Dennoch werden im Folgenden die Relaxationszeiten doppeltlogarithmisch gegen die quadratische Massenanzahl $z^{2}$ aufgetragen. Falls die Näherungsgleichung gilt, sollten in dieser Auftragung die Relaxationszeiten $\tau_{p}$ der jeweils $p$-ten Mode auf einer Geraden der Steigung $m=1$ liegen. Entscheidend ist dabei, dass die Geradensteigung unabhängig von der Größe der Kettensegmente ist. Eine veränderte Segmentgröße ändert daher lediglich die Skalierung der Abszisse. Diese Eigenschaft erscheint vorteilhaft, solange die tatsächliche Größe der unabhängigen Rouse-Segmente unbekannt ist.

Abbildung 4.12 zeigt die doppeltlogarithmische Auftragung für die jeweils größte und, soweit vorhanden, zweit- und drittgrößte Relaxationszeit der verschiedenen PEG-Schmelzen aus Tabelle 4.2. Zusätzlich sind die Werte für PPG 1000 mit eingetragen, dessen Parameter denen der PEG-Schmelzen sehr ähnlich sind. Dabei wurde vorläufig jedes Kohlenstoff- und Sauerstoffatom als rotationsfähige Einheit aufgefasst und als Masse gezählt³.

Die jeweils größten Relaxationszeiten liegen in guter Näherung auf einer Geraden. Die Steigung dieser Geraden wurde durch lineare Regression ermittelt. Sie beträgt $m=1.117$ mit einem Fehler von $\Delta m=0.067$ und stimmt somit recht gut mit der theoretischen Vorhersage überein. Für die jeweils zweitgrößten Relaxationszeiten ergibt die lineare Regression $m=0.95 \pm 0.23$, was ebenfalls gut dem erwarteten Wert entspricht. Der Fehler dieser Regression ist allerdings deutlich höher, was vor allem auf den Wert von PEG $300\left(z^{2}=408\right)$ zurückzuführen ist. Da alle drei Relaxationszeiten von PEG 300 deutlich oberhalb der Ausgleichsgraden liegen, kann vermutet werden, dass hier ein systematischer Fehler bei der Beschreibung des Absorptionsspektrums aufgetreten ist. Eine erneute Regressionsanalyse zeigt, dass eine Beschreibung dieses Spektrums mit vorgegebener Relaxationszeit $\tau_{\mathrm{D} 3}=0.5 \mathrm{~ns}$ und gleichem $B$-Wert möglich ist, die zu einer Verringerung der beiden anderen Relaxationszeiten führt $\left(\tau_{\mathrm{D} 2}=3.02 \mathrm{~ns}\right.$ anstatt $5.2 \mathrm{~ns}, \tau_{\mathrm{D} 4}=0.130 \mathrm{~ns}$ anstatt $0.144 \mathrm{~ns}$, siehe auch Tab.4.3). Die Steigung der Ausgleichsgeraden für die drittgrößten Relaxationszeiten ändert sich allerdings mit dem etwas kleineren Wert von $\tau_{\mathrm{D} 4}$ kaum. Sie weicht deutlich von der Vorhersage des Modells ab.

Ein möglicher Grund für diese Abweichung könnte eine falsche Modenzuordnung oder eine unzureichende Beschreibung der Absorptionsspektren sein. Dies würde

\footnotetext{
${ }^{3}$ Nebengruppen bei PPG 1000 werden nicht mitgezählt.
} 


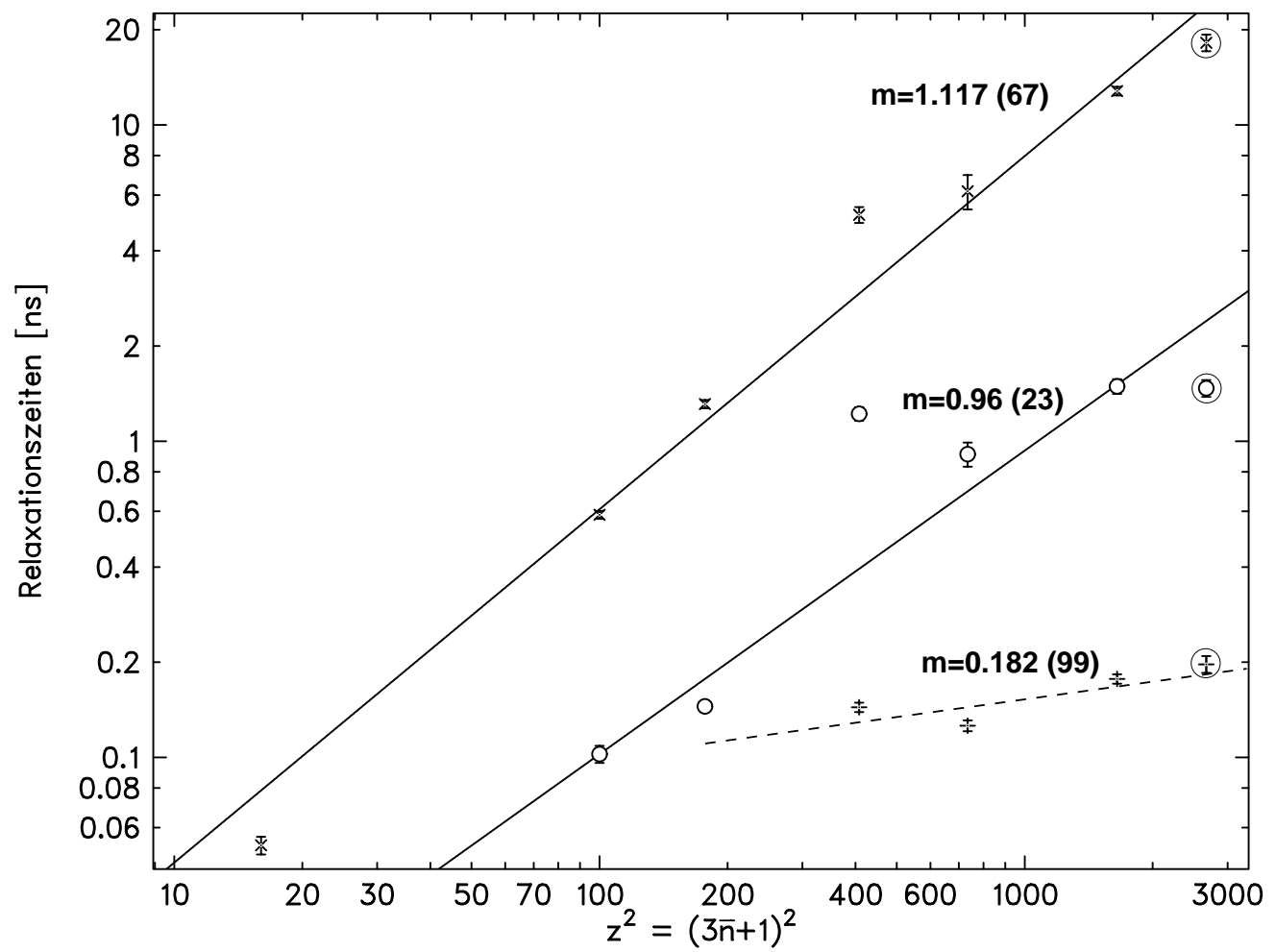

Abbildung 4.12: Größte (×), zweitgrößte (o) und drittgrößte (+) Relaxationszeit der PEG- und PPG-Schmelzen aus Tab.4.2, aufgetragen über dem Quadrat der Zahl der rotationsfähigen Gruppen $z^{2}$, wobei jedes Kohlenstoff- bzw. Sauerstoffatom als eine solche Gruppe betrachtet wird. Daraus folgt für PEG und PPG $z^{2}=(3 \bar{n}+1)^{2}$ mit der mittleren Anzahl monomerer Einheiten $\bar{n}$ für die jeweilige Schmelze (s. auch Tab.2.1). Eingekreiste Punkte gehören zu PPG 1000. Durchgezogene/gestrichelte Linien: Ergebnis der linearen Regression, $m$ : Steigung der Regressionsgeraden. 
sich mit der bereits geäußerten Vermutung decken, dass den Spektren der längeren Oligomerketten (Molmasse $400 \mathrm{~g} / \mathrm{mol}$ und höher) möglicherweise mehr als drei Einzelzeitrelaxationen zugrunde liegen. Besonders im Fall der höheren, im logarithmischen Maßstab eng liegenden Moden ist es vorstellbar, dass eine oder mehrere Einzelzeitrelaxationen bei der Regressionsanalyse in einem Mischterm zusammengefasst werden.

Eine wichtige Frage ist, wieviele Moden bei den jeweiligen Schmelzen zu erwarten sind. Eine grobe Abschätzung ist durch den Vergleich von Molmasse und der Anzahl der auftreten Moden für die Schmelzen mit Kettenlänge $\bar{n}=1$ bis $\bar{n}=6.7$ möglich (Tab.4.2): Bei einer Zunahme der Molmasse um etwa $100 \mathrm{~g} / \mathrm{mol}$ kommt jeweils eine weitere Mode hinzu. Bei PEG 400 sollten somit vier, bei PEG 600 sechs, bei PPG 1000 - vorausgesetzt es verhält sich wie PEG - sogar 10 Moden angeregt werden können. Es ist jedoch abzusehen, dass eine Beschreibung der Absorption in dem vorliegenden Frequenzbereich mit derart vielen Debye-Spektralfunktionen zu einer Überbestimmung der Spektren führen wird. Daher wurde bei einer erneuten Regressionsanalyse der PEG- und PPG-Spektren zunächst nur jeweils eine weitere Debye-Funktion hinzugenommen. Um dabei unsinnige Parameterwerte zu vermeiden, wurde der $B$-Wert auf 150 ps festgehalten. Er entspricht damit etwa den $B$-Werten aus der weitgehend freien ersten Anpassung der Schmelzen (Tab.4.2). Im Fall von PEG 400 (II) musste außerdem eine Relaxationszeit fest vorgegeben werden, um sinnvolle Funktionsparameter zu erhalten. Das Ergebnis der Anpassung findet sich in Tabelle 4.3. Eine zu Abbildung 4.12 analoge Auftragung dieser Daten ist in Abb. 4.13 zu sehen.

Die Geraden, die sich für die größten und zweitgrößten Relaxationszeiten ergeben, verlaufen jetzt nahezu parallel. Ihre Steigungen liegen beide leicht oberhalb des vom Modell vorhergesagten Wertes. Deutlich geändert hat sich der Verlauf der jeweils drittgrößten Relaxationszeiten. Sie folgen nun ebenfalls einer Geraden, deren Steigung dicht an $m=1$ liegt. Dies bestätigt die Vermutung, dass die Spektren von PEG 400, PEG 600 und PPG 1000 bei der ersten Analyse der Daten durch zu wenige Einzelzeitrelaxationen beschrieben wurden. Vor allem aber sprechen diese Ergebnisse dafür, dass die Einzelzeitrelaxationen als Eigenmoden der Kettenbewegung im Sinne des Rouse-Modells interpretiert werden können. Die etwas geringere Steigung der dritten Geraden und der deutlich schwächere Anstieg der vierten Geraden kann dadurch erklärt werden, dass für eine korrekte Beschreibung der Spektren von PEG 600 und PEG 1000 noch weitere Debye-Spektralfunktionen notwendig wären. Für eine Regression mit fünf oder mehr Debye-Spektralfunktionen ist der untersuchte Frequenzbereich jedoch zu schmal. Bereits bei der Vier-DebyeDarstellung musste mindestens ein Parameter festgehalten werden, um eine stabile Anpassung und konsistente Parameterverläufe zu erhalten. Die Einführung weiterer Freiheitsgrade würde die Unsicherheiten in den Funktionsparametern zu sehr erhöhen und keine verlässlichen Anpassungsergebnisse mehr liefern. Aus diesem Grund wurde auf eine Regression mit mehr als vier Debye-Spektralfunktionen verzichtet. Die damit verbundenen systematischen Fehler, besonders in den Parametern der hochfrequenten Einzelzeitrelaxationen, sind dementsprechend bei der 


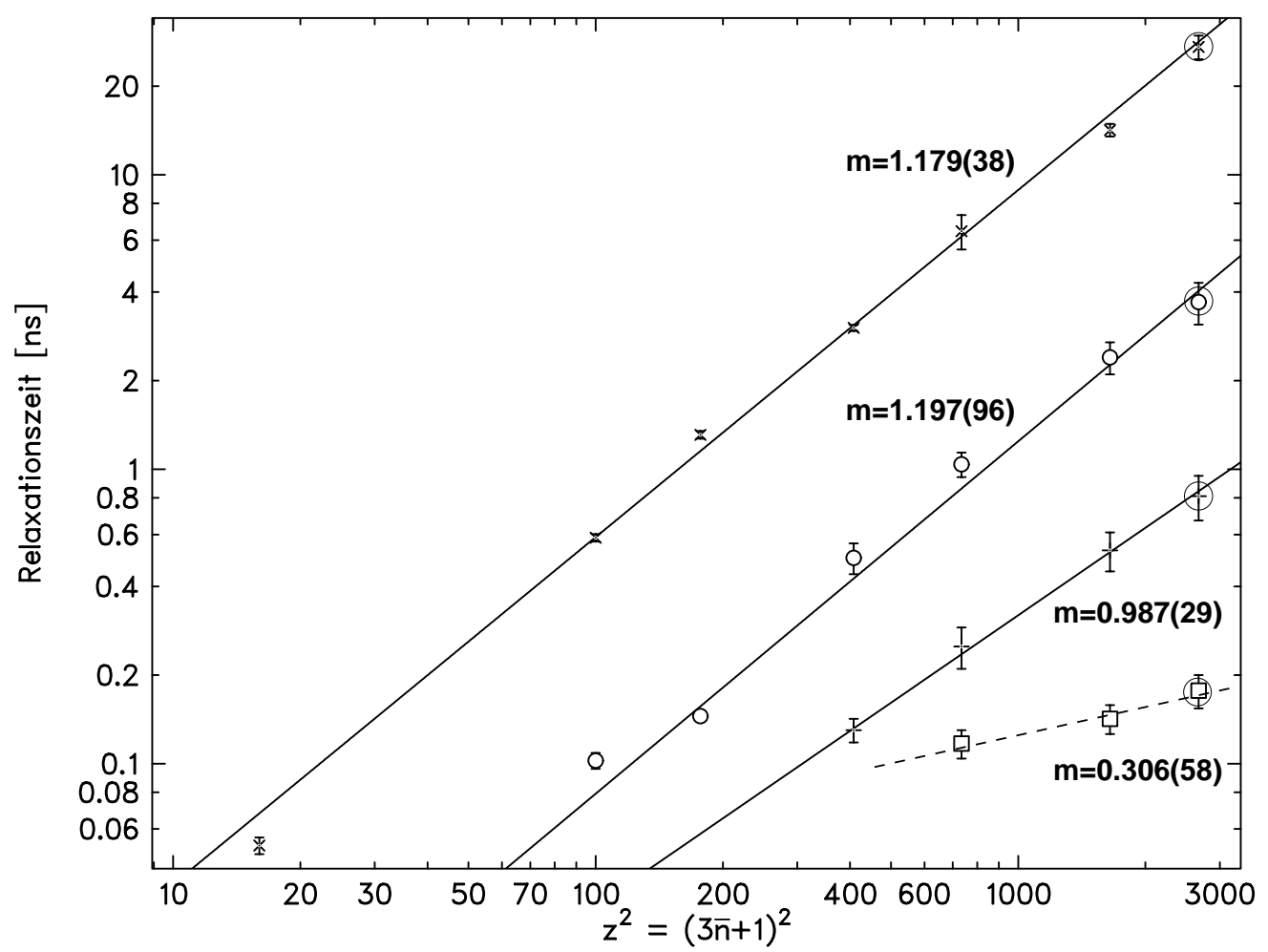

Abbildung 4.13: Größte $(\times)$, zweitgrößte (o), drittgrößte $(+)$ und viertgrößte $(\square)$ Relaxationszeit der PEG- und PPG-Schmelzen nach Tab.4.3, aufgetragen über dem Quadrat der Massenanzahl $z^{2}$ wie in Abb.4.12. Eingekreiste Punkte gehören zu PPG 1000. Durchgezogene/gestrichelte Linien: Ergebnis der linearen Regression, $m$ : Steigung der Regressionsgeraden. 


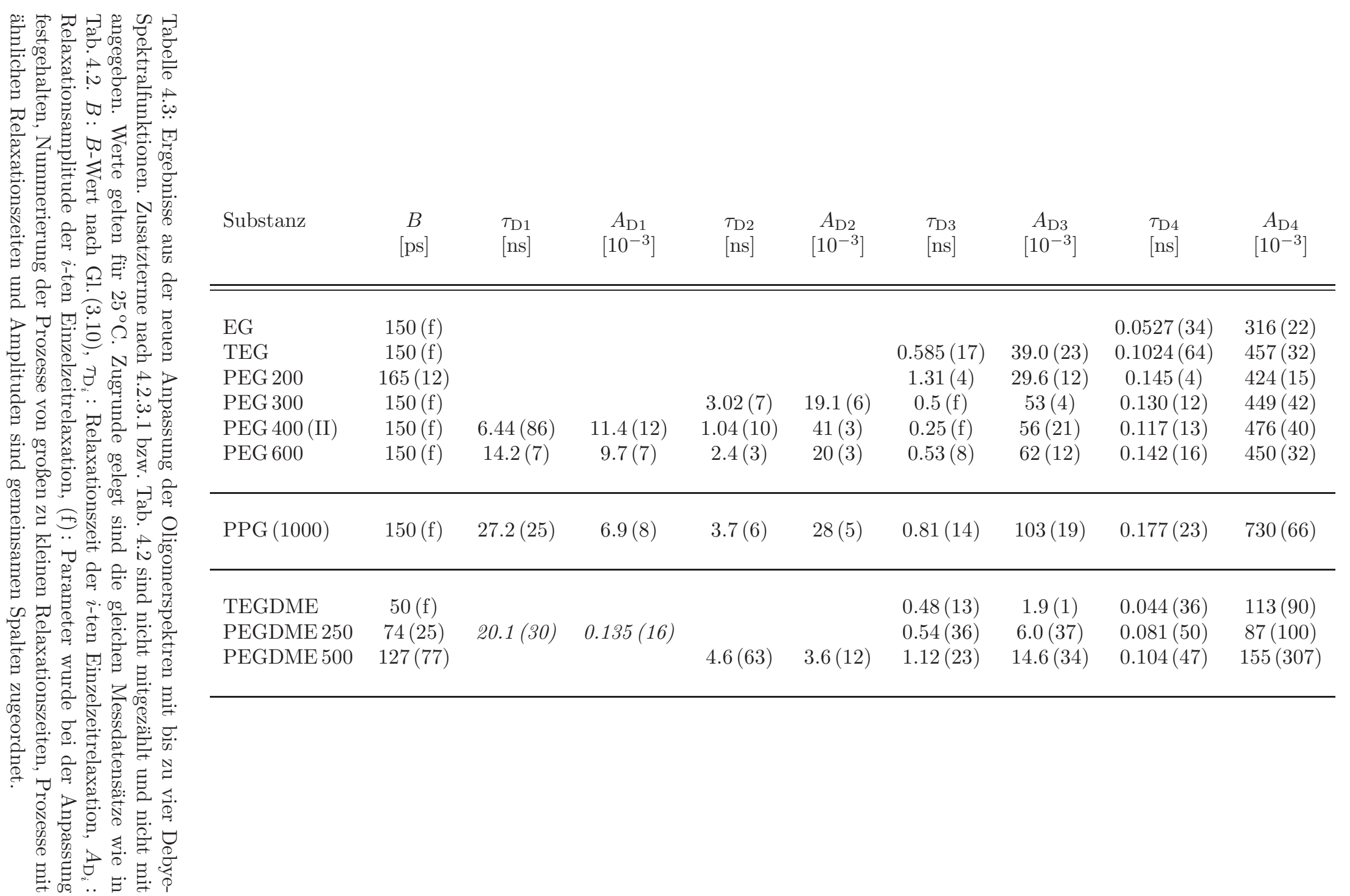


weiteren Diskussion zu berücksichtigen.

\subsubsection{Die Ordnung der auftretenden Moden}

Bislang ist ungeklärt, um welche Moden es sich bei den Einzelzeitrelaxationen eigentlich handelt. Aufschluss darüber kann der senkrechte Abstand der Geraden, d.h. der Faktor zwischen den Relaxationszeiten geben, denn nach der Näherungsgleichung (4.7) nehmen die Relaxationszeiten mit dem Quadrat der Modennummer $p$ ab. Bei gleichbleibender Kettenlänge ist also die Relaxationszeit der Grundmode um eine Faktor 4 höher als die der zweiten Mode, um einen Faktor 9 höher als die der dritten usw.

Vor allem aufgrund der unzureichenden Beschreibung der Spektren von PEG 600 und PPG 1000 verlaufen die Geraden der einzelnen Moden in Abb. (4.13) nicht parallel. Um dennoch eine grobe Abschätzung für die Modennummern zu erhalten, wurde eine erneute Regression der Daten durchgeführt, bei der die Werte von PEG 600 und PPG 1000 nicht berücksichtigt wurden. Bei den drittgrößten Relaxationszeiten bleiben dann nur zwei Werte übrig, so dass kein Regressionsfehler angegeben werden kann. Im Fall der viertgrößten Relaxationszeiten ist nur noch der Wert von PEG 400 vorhanden. Abbildung 4.14 zeigt das Ergebnis der Anpassung sowie die Relaxationszeitverhältnisse von Grundmode und höheren Moden. Für die viertgrößte Relaxationszeit wurde als Hilfslinie für das Auge eine Gerade mit Steigung $m=1$ durch den Punkt von PEG 400 vorgegeben. Da die Geraden weiterhin nicht vollständig parallel liegen, wurde ihr Abstand (außer im Fall der Hilfsgeraden) für verschiedene Kettenlängen bestimmt.

Die Zahlenverhältnisse lassen folgenden Schluss zu: Die Einzelzeitrelaxation mit der größten Relaxationszeit wird der ersten Rouse-Mode zugeordnet. Ihre Relaxationszeit wird im Folgenden mit $\tau_{1}$ bezeichnet. Bei der sich anschließenden Relaxation handelt es sich vermutlich um die dritte Mode $\left(\tau_{1} / \tau_{3}=9\right)$. Das Zahlenverhältnis 24.17 bis 29.39 lässt vermuten, dass die nächst höhere Mode von der Ordnung $p=5$ ist. Das Relaxationszeitverhältnis der höchsten aufösbaren Mode und der Grundmode, berechnet nur für PEG 400 und damit sehr fehlerbehaftet, scheint am ehesten zu der siebten Rouse-Mode $\left(\tau_{1} / \tau_{7}=49\right)$ zu passen. Trägt man die gemessenen Relaxationszeiten als Funktion der so zugeordneten Modennummer auf, ergibt sich in guter Näherung die von Rouse vorhergesagte Relation $\tau \sim p^{-2}$ (Abb.4.15).

Offenbar treten also nur die ungeraden Moden auf. Dabei ist allerdings unklar, ob die geraden Moden gar nicht angeregt werden, oder ob sie mit Hilfe der Ultraschallabsorptionsspektroskopie nur nicht detektiert werden können, weil der ihnen zugrunde liegende molekulare Prozess nicht mit einer maßgeblichen Volumen- oder Enthalpieänderung verbunden ist und somit nicht an das Schallfeld koppelt.

Ein denkbarer Grund dafür, dass eine Mode gar nicht auftreten kann, wäre die Blockade einer bestimmten Bewegung innerhalb des Moleküls, beispielsweise durch seinen sterischen Aufbau. Bei den vergleichsweise frei drehbaren KohlenstoffKohlenstoff und Sauerstoff-Kohlenstoff-Bindungen ist eine solche Blockade aber 


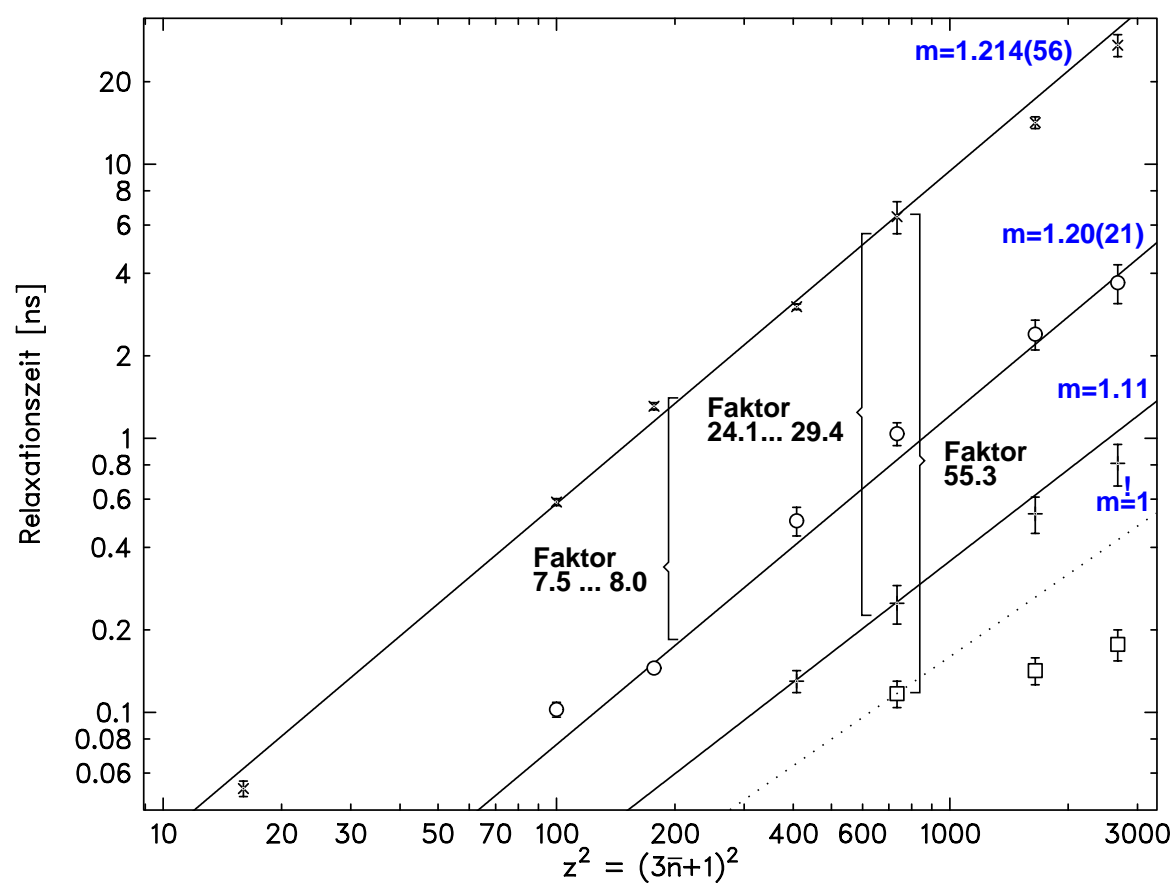

Abbildung 4.14: Abschätzung der Relaxationszeitverhältnisse zur Bestimmung der Modennummer. Bedeutung der Symbole wie in Abb.4.13, Bei der linearen Regression (durchgezogene Linien) wurden die Relaxationszeiten von PEG 600 und PPG 1000 nicht berücksichtigt. Die Hilfslinie (gepunktet) für die hochfrequenteste Einzelzeitrelaxation entspricht einer Geraden mit Steigung $m=1$.

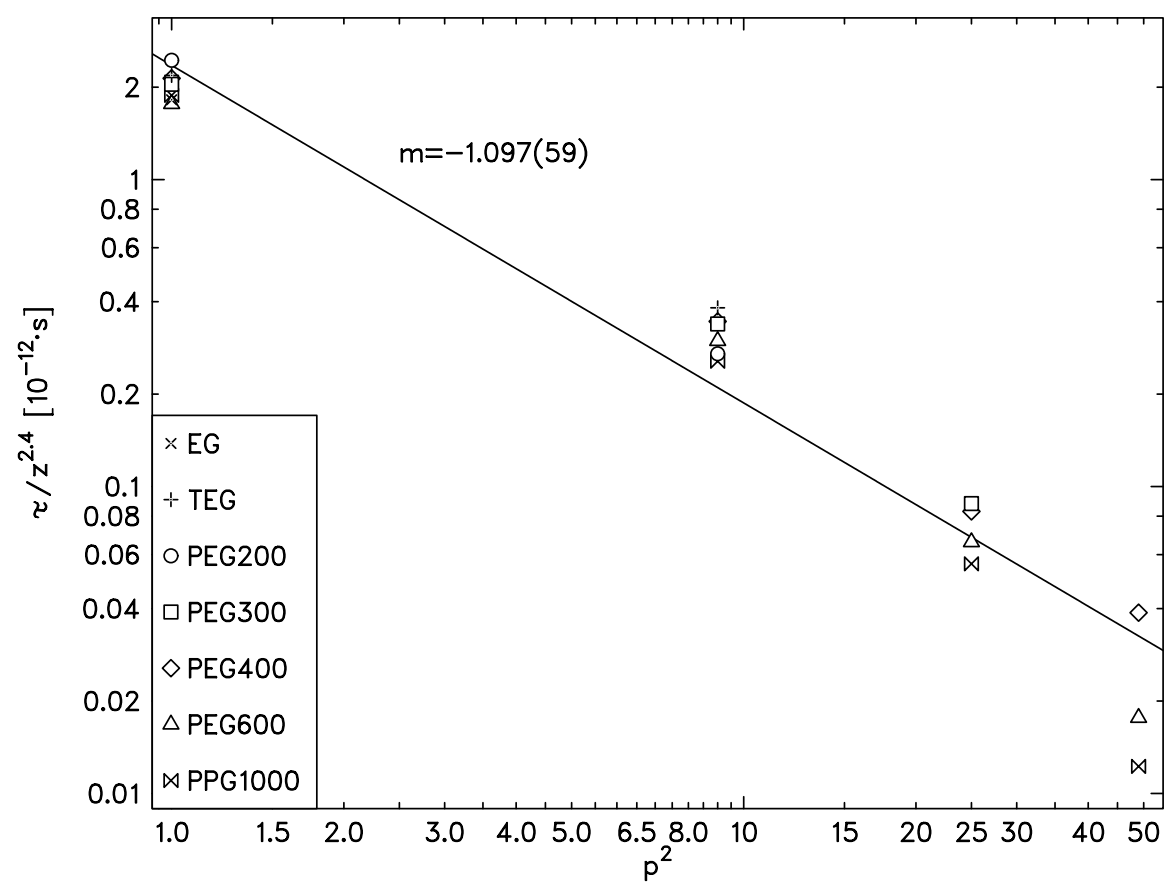

Abbildung 4.15: Abhängigkeit der Relaxationszeit von der Modenordnung unter Berücksichtigung der beobachteten Kettenlängenabhängigkeit $\tau \sim z^{2.4}$ (vergl. Abb.4.14). Deutliche Abweichungen von der Vorhersage $\tau \sim p^{-2}$ des Rouse-Modells zeigen nur die höchsten Moden von PEG 600 und PPG 1000 . 
nur schwer vorstellbar. Die Wasserstoffbrückenbindungen, die zwischen den PEGMolekülen gebildet werden können, sind zu kurzlebig (dielektrische Relaxationszeit in Wasser: $10 \mathrm{ps}$ ), als dass sie die Konformationsänderung einer Polymereinheit vollständig unterdrücken könnten. Sie werden eher zu einer Verlangsamung der Prozesse führen (s. auch Abschnitte 4.3.2.4, 6 und 7). Gegen eine Blockade bestimmter Bewegungen aufgrund des molekularen Aufbaus spricht außerdem, dass bei PEG - unabhängig von der Kettenlänge - stets die gleichen Moden wegfallen. Da die Anzahl der chemischen Gruppen, die eine Blockade bewirken könnten, mit der Kettenlänge zunimmt, wäre aber zu erwarten, dass je nach Kettenlänge unterschiedliche Moden behindert werden.

Wahrscheinlicher ist, dass sich die Moden mit gerader Ordnungszahl der Beobachtung durch die Ultraschallabsorptionsspektroskopie entziehen, weil sie ohne eine Änderung des Gesamtvolumens eines Moleküls ablaufen. Dies könnte dann der Fall sein, wenn die Moden eine hauptsächlich longitudinale Kettenbewegung beschreiben: Wenn man von einer longitudinalen Auslenkung der Massen aus ihren Ruhelagen ausgeht, wird sich bei den geraden Moden an der Gesamtlänge der Kette nichts ändern. Dies wird am Beispiel der zweiten und dritten Mode deutlich (Abb. 4.10, 4.11). Bei der zweiten Mode verkürzt sich zwar die eine Hälfte der Kette, gleichzeitig wird aber die andere Hälfte in gleichem Maße gestreckt, so dass die Gesamtlänge des Moleküls im Vergleich zur Gleichgewichtslage erhalten bleibt. Bei der dritten Mode hingegen tritt eine effektive Längenänderung auf, da hier zwei Teilstücke des Polymers gestreckt werden, während sich nur ein Teilstück verkürzt.

Stellt man sich das Polymer entsprechend dem Rouse-Modell als eine ausreichend lange Kette punktförmiger Massen und longitudinal auslenkender Federn vor, ist diese Erklärung plausibel. Bei den kurzen Oligomerketten, deren Konformationsänderungen durch Rotation um ihre Bindungen mit vergleichsweise festem Bindungsabstand erzeugt werden, erscheint es jedoch schwierig, den Volumenbedarf verschiedener Rotationsisomere in ihrer polymeren Umgebung vorherzusagen und sich die Moden als eine longitudinale Expansion und Kompression der Moleküle vorzustellen. Dennoch weisen die experimentellen Ergebnisse eher auf eine derartige Bewegung hin. Simulationsrechungen könnten hier möglicherweise zu einem genaueren Verständnis und einer verbesserten Anschauung der molekularen Vorgänge führen.

\subsubsection{Abschätzung der Segmentlänge}

Bislang wurde als Segmentlänge der Abstand der rotationsfähigen Kohlenstoffbzw. Sauerstoffgruppen angenommen. Mit Hilfe der im letzten Abschnitt ermittelten Ordnung der beobachteten Moden kann nun eine Abschätzung der tatsächlichen Segmentlänge vorgenommen werden. In Tabelle 4.4 findet sich eine Gegenüberstellung der Anzahl der rotationsfähigen Einheiten und der beobachteten Moden für die verschiedenen PEG- und PPG-Schmelzen.

Im Mittel nimmt die Kettenlänge um etwa sieben rotationsfähige Gruppen zu, 


\begin{tabular}{l|c|l} 
Molekül & $\begin{array}{c}\text { rotationsfähige Gruppen } \\
(3 \bar{n}+1)\end{array}$ & beobachtete Moden \\
\hline \hline & & \\
EG & 1 & 1. \\
TEG & 13.3 & $1 ., 3$. \\
PEG 200 & 20.2 & $1 ., 3$. \\
PEG 300 & $1 ., 3 ., 5$. \\
PEG 400 & 27.1 & $1 ., 3 ., 5 ., 7$. \\
PEG 600 & 40.6 & $1 ., 3 ., 5 ., 7 .,(9 ., 11 . ?)$ \\
PEG 1000 & 51.7 & $1 ., 3 ., 5 ., 7 .,(9 ., 11 ., \ldots ?)$
\end{tabular}

Tabelle 4.4: Gegenüberstellung der Anzahl der rotationsfähigen Einheiten und der beobachteten Moden für die verschiedenen PEG- und PPG-Schmelzen. $\bar{n}$ : mittlere Anzahl monomere Einheiten. Jedes Kohlenstoff- und Sauerstoffatom zählt als rotationsfähige Einheit. Nebengruppen bei PPG 1000 werden dabei nicht berücksichtigt.

bevor eine neue, ungerade, Mode auftritt. Dies bedeutet, dass etwa drei bis vier Kohlenstoff- bzw. Sauerstoffatome ein Kettensegment bilden, was ungefähr der Länge einer monomeren Einheit des PEG-/PPG-Moleküls entspricht. Das Ethylenglykolmolekül entspricht demnach etwa einem Segment, das Triethylenglykol einem System von ca. drei Gliedern und damit vier Massen.

Für eine genauere Bestimmung der Segmentlänge wird nun PEG 200 betrachtet: Unter der Annahme, dass nur ungerade Moden im Spektrum auftreten, muss TEG mindestens drei, PEG 200 darf höchstens vier Segmente umfassen. Aus der obigen Abschätzung lässt sich damit für PEG 200 eine Länge von vier Segmenten und damit eine Segmentlänge von ca. 3.3 rotationsfähigen Gruppen ermitteln. Daraus ergeben sich für EG, TEG, PEG 200, PEG 300 und PEG 400 Längen von 1.2, 3.0, 4.0, 6.1 und 8.1 unabhängigen Segmenten, in Übereinstimmung mit der beobachteten Zahl der Moden. Da in den gemessenen Ultraschallspektren maximal vier (ungerade) Moden aufgelöst werden konnten, können die für PEG600 und 1000 bestimmten Längen (etwa 12 bzw. 16 Segmente) nicht anhand der Zahl beobachteter Moden überprüft werden.

Die ermittelte Segmentlänge stimmt sehr gut mit den Vorhersagen des DTOModells überein, wonach ein unabhängiges Kettensegment durch eine chemische Gruppe von zwei bis drei rotationsfähigen (C-C-)Bindungen gebildet wird [8]. Hingegen werden die Abschätzungen der Segmentlänge durch das Rouse-Modell (Abschnitt 4.3.1) deutlich schlechter erfüllt, woraus geschlossen werden kann, dass die Kettendynamik eher als Torsionsbewegung denn als Abstandsänderung atomarer Gruppen aufzufassen ist.

\subsubsection{Vergleich von PEG mit PEGDME und n-Alkanen}

Um zu untersuchen, inwieweit sich die bei PEG beobachtete Modenstruktur auch in den Ultraschallabsorptionsspektren anderer Oligomere wiederfinden lässt und 


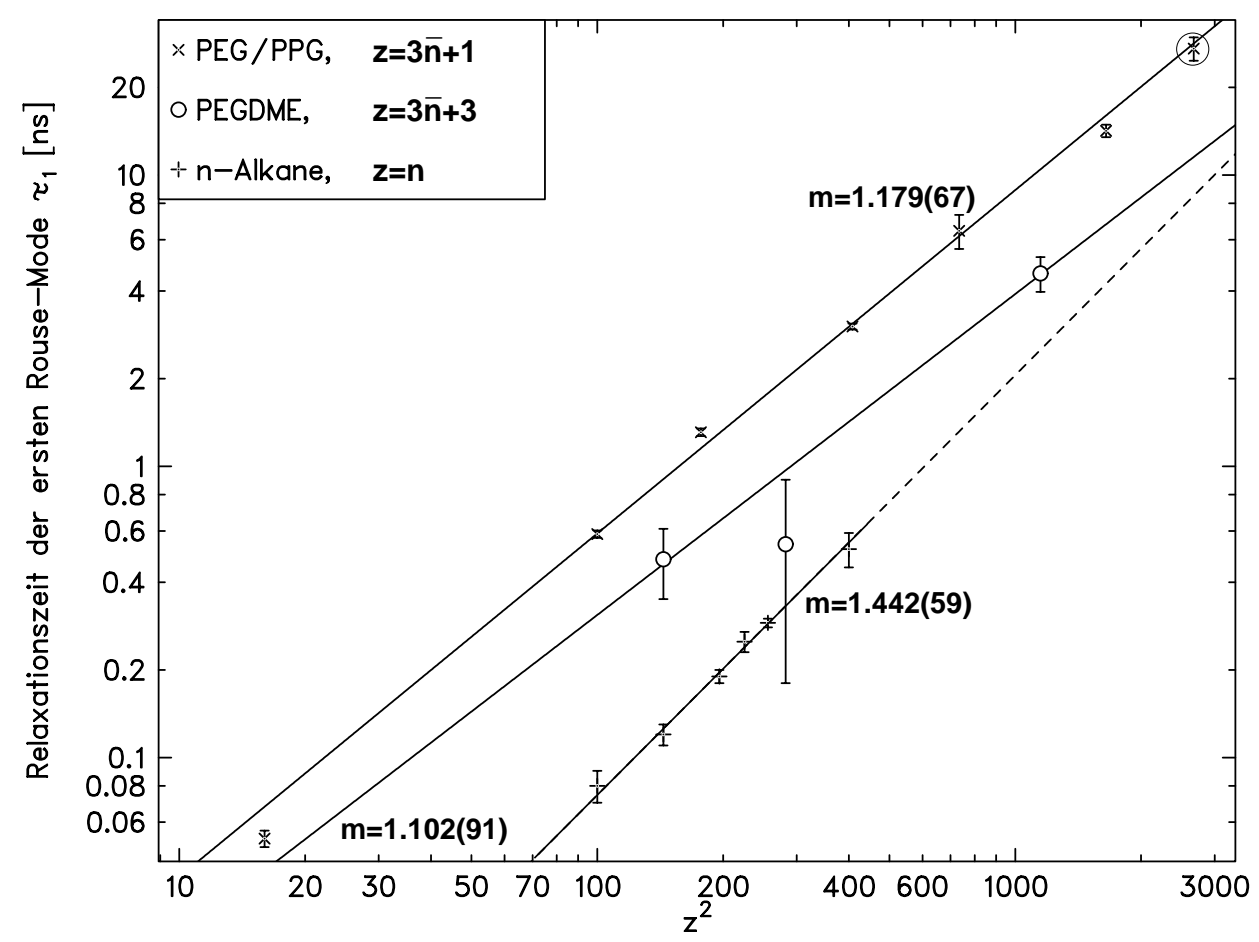

Abbildung 4.16: Relaxationszeit $\tau_{1}$ der ersten Rouse-Mode für PEG, PEGDME und n-Alkane in Abhängigkeit von der quadratischen Anzahl $z^{2}$ der rotationsfähigen molekularen Einheiten. Die entsprechende Relaxationszeit von PPG 1000 (eingekreist) wurde wieder mit einbezogen. $n$ : Zahl der Kohlenwasserstoffgruppen, $\bar{n}$ : mittlere Anzahl monomerer Einheiten. Durchgezogene Linien: Regressionsgeraden, $m$ : Steigung der Geraden. Gestrichelte Linie: Hilfslinie - in diesem Kettenlängenbereich liegen reine n-Alkane nur noch in festem Zustand vor.

um einen Eindruck zu gewinnen, wie der molekulare Aufbau der Substanz die Modendynamik beeinflusst, wurden die Relaxationszeiten der PEG-Schmelzen mit denen von Ethylenglykoldimethylether-Oligomeren und n-Alkanen verglichen.

Die Ultraschallspektren kurzkettiger n-Alkane zeigen einen hochfrequenten Relaxationsprozess, der der ersten vom DTO-Modell vorhergesagten Torsionsmode zugeordnet wird [51]. Die Relaxationszeit dieser Grundmode ist in Abbildung 4.16 zusammen mit der entsprechenden Relaxationszeit für die PEG-Schmelzen als Funktion der quadratischen Anzahl der rotationsfähigen molekularen Einheiten $z^{2}$ dargestellt. Außerdem ist die jeweils tieffrequenteste Relaxationszeit ${ }^{4}$ aus der analytischen Beschreibung der Oligoethylenglykoldimethylether-Spektren (Tab. 4.3) mit aufgetragen. Dabei wurde zunächst wieder jeder Ethersauerstoff sowie jede Methyl- und Hydroxidgruppe als rotationsfähige Einheit gezählt.

Auch die Relaxationszeiten der Alkane liegen in der doppeltlogarithmischen Skalierung auf einer Geraden. Neben ihrer im Vergleich zu den Oligoethylenglykolen etwas höheren Steigung fällt vor allem der deutlich kleinere Ordinatenabschnitt der Geraden auf.

Ein möglicher Grund für die langsamere Modendynamik der Oligoethylenglykole

\footnotetext{
${ }^{4}$ ausgenommen Zusatzterme nach 4.2 .3 .1
} 


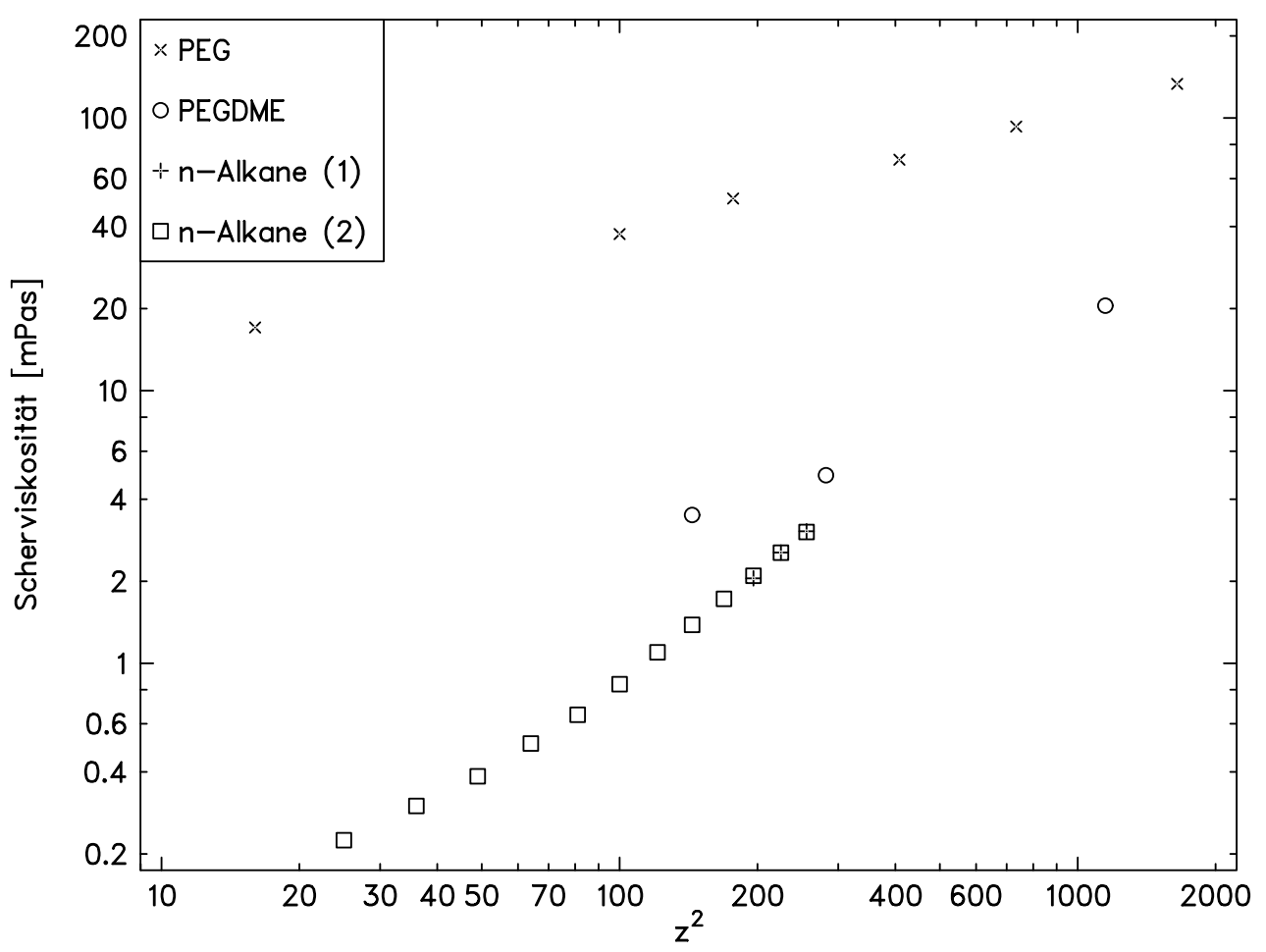

Abbildung 4.17: Scherviskosität $\eta_{\mathrm{s}}$ von PEG, PEGDME und n-Alkanen bei $25^{\circ} \mathrm{C}$ in Abhängigkeit von der quadratischen Anzahl $z^{2}$ der rotationsfähigen molekularen Einheiten. $z$ wie in Abb.4.17, (1) : nach [51] (2): interpoliert aus Daten nach [52].

ist, dass sie im Gegensatz zu den unpolaren Alkanen über die freien Elektronenpaare der Sauerstoffatome und die Wasserstoffatome an den OH-Endgruppen Wasserstoffbrückenbindungen eingehen können. Es ist anzunehmen, dass eine derartige Assoziation zu einer Erhöhung der viskosen Reibung und damit nach dem RouseModell zu einer Zunahme der Relaxationszeit führt. Tatsächlich beobachtet man bei den PEG-Schmelzen eine höhere Viskosität als bei den Alkanen (Abb. 4.17). Auch im DTO-Modell ist eine Relaxationszeiterhöhung aufgrund der Vernetzung zu erwarten, da man davon ausgehen kann, dass eine Wasserstoffbrückenbindung die Potentialbarriere zwischen verschiedenen Zuständen der Torsionsoszillatoren erhöht. Da der Vernetzungsgrad mit zunehmender Kettenlänge abnimmt (vergl. Abschnitt 6.2), sollte der Einfluss der Assoziation auf die Relaxationszeit für längere PEG-Ketten geringer werden. Ein Indiz dafür ist die etwas kleinere Steigung der PEG-Geraden gegenüber der Alkan-Geraden in Abb. 4.16-eine Tendenz, die sich ebenfalls im Verlauf der Scherviskositäten wiederfinden lässt.

Dass nicht allein die Vernetzung über Wasserstoffbrückenbindungen für die Relaxationszeiterhöhung verantwortlich ist, zeigen die Messergebnisse für die PEGDMESchmelzen. Die monomere Einheit dieses Oligomers, $\left(\mathrm{CH}_{2}-\mathrm{O}-\mathrm{CH}_{2}\right)$, entspricht der von PEG, wegen seiner unpolaren Methyl-Endgruppen ist es jedoch in einer reinen Schmelze nicht in der Lage, Wasserstoffbrückenbindungen auszubilden. Da lediglich drei verschiedene Kettenlängen von PEGDME untersucht wurden und die Anpas- 
sungsparameter hohe Unsicherheiten aufweisen, ist hier eine lineare Regression nur mit großen Fehlern möglich. Dennoch fügen sich die Werte sinnvoll in das Gesamtbild ein: Die Steigung der Regressionsgeraden in Abb. 4.16 liegt mit $m=1.1$ auch für PEGDME dicht an den Vorhersagen des Modells, wobei für gleiche Kettenlängen die Relaxationszeit von PEGDME immer größer als die des n-Alkans, aber kleiner als die des PEG-Moleküls ist. Offenbar führen also bereits die veränderten stereochemischen Eigenschaften der Kohlenstoff-Sauerstoff-Verbindung gegenüber einer reinen Kohlenwasserstoffkette zu einer Verlangsamung der Dynamik. Im Gegensatz zur Wasserstoffbrückenassoziation bei PEG beeinflussen sie die Scherviskosität aber kaum (Abb. 4.17), so dass eine veränderte viskose Reibung als Erklärung für die Relaxationszeiterhöhung nicht ausreicht. Da sich nach [53] die Rotationsbarriere einer $\mathrm{H}_{3} \mathrm{C}-\mathrm{OCH}_{3}$-Bindung gegenüber einer $\mathrm{H}_{3} \mathrm{C}-\mathrm{C}_{2} \mathrm{H}_{5}$-Bindung absenkt, wäre auch im DTO-Modell eher eine Abnahme der Relaxationszeit zu erwarten. Hier müssen demnach noch andere Mechanismen für die Verlangsamung der Modendynamik verantwortlich sein. Im Rouse-Modell könnte ein größerer mittlerer quadratischer End-zu-End-Abstand der Kettensegmente, im DTO-Modell eine verringerte Torsionsfrequenz zu einer kleineren Federhärte der Kettensegmente und damit zu einer Relaxationszeiterhöhung führen. Da aber weder für den mittleren quadratischen End-zu-End-Abstand noch für die die Torsionsfrequenz vergleichbare Messungen an PEGDME- und Alkan-Schmelzen bekannt sind, kann über die Federhärte keine Aussage gemacht werden.

Bis hierher wurde nur die Grundmode von PEG, PEGDME und n-Alkanen betrachtet. Während bei PEG und PEGDME auch höhere Moden im untersuchten Spektralbereich gefunden wurden (Tab. 4.3), werden in [51] keine weiteren Relaxationsprozesse bei den n-Alkanen erwähnt. Vergleicht man jedoch die Kettenlänge der Oligoethylenglykole und der n-Alkane mit der Anzahl der auftretenden Moden, so würde man - ähnliche Segmentlängen vorausgesetzt - für Dekan und alle längeren flüssigen Alkane ebenfalls eine weitere Mode erwarten. Für Tetra-, Penta- und Hexadekan ( $z=14,15$ bzw. 16) wurde anhand des Relaxationszeitverhältnisses der beiden ersten Moden von PEG $200(z=13.3)$ die Lage der nächst höheren Moden abgeschätzt. Ihre Relaxationszeit sollte für Hexadekan bei etwa 0.03 ns liegen, das entspricht einer Relaxationsfrequenz von etwa $5 \mathrm{GHz}$. Da die Schallabsorption aber nur bis $2 \mathrm{GHz}$ gemessen wurde, ist davon auszugehen, dass die Relaxationstufe der nächsten Mode kaum noch detektiert werden kann. Das gilt erst recht für kürzere Kettenlängen. Dennoch wurde versucht, die drei genannten Spektren durch zwei Debye-Spektralfunktionen zu beschreiben, wobei für die Relaxationszeit der zweiten Mode der Wert aus der Abschätzung fest vorgegeben wurde. Dass Ergebnis dieser Regression unterstützt die Vermutung, dass höhere Moden zwar auftreten, durch die Ultraschallabsorptionsmessungen aber nicht mehr verlässlich beobachtet werden können.

Der hochfrequente Bereich der Penta- und Hexadekan-Spektren wird durch die Hinzunahme einer weiteren Einzelzeitrelaxation besser beschrieben (vergl. Abb. 4.18). Dabei ergeben sich trotz der erhöhten Unsicherheiten in den freien Parametern für beide Spektren in der Regressionsanalyse stabile und sinnvolle Werte, 

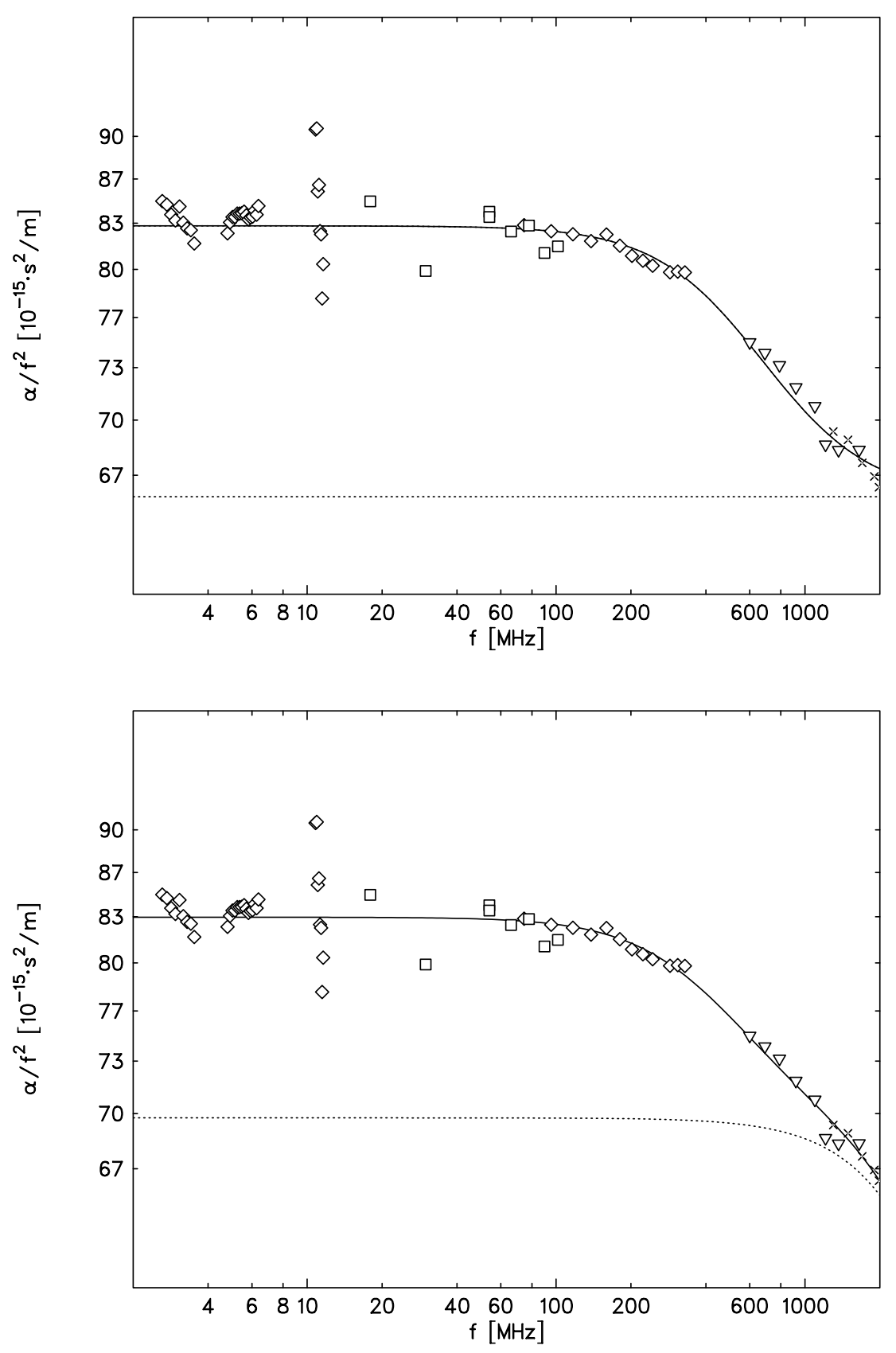

Abbildung 4.18: Messwerte sowie Ein-Debye-Darstellung (oben) und Zwei-Debye-Darstellung (unten) des Absorptionsspektrums von Pentadekan bei $25^{\circ} \mathrm{C}$. Durchgezogene Linien: Summe von Debye-Funktionen und Hintergrundsanteil. Gestrichelte Linien: $B / c_{\mathrm{s} \infty}$ (oben) bzw. $B / c_{\mathrm{s} \infty}+$ hochfrequenter Debye-Term (unten). 


\begin{tabular}{c|ccccc} 
Substanz & $B[\mathrm{ps}]$ & $\tau_{\mathrm{D} 1}[\mathrm{~ns}]$ & $A_{\mathrm{D} 1}\left[10^{-3}\right]$ & $\tau_{\mathrm{D} 2}[\mathrm{~ns}]$ & $A_{\mathrm{D} 2}\left[10^{-3}\right]$ \\
\hline \hline \multirow{3}{*}{ Tetradekan } & $78.6(7)$ & $0.190(10)$ & $16.9(15)$ & & \\
& $45(\mathrm{f})$ & $0.204(14)$ & $13.9(14)$ & $0.02(\mathrm{f})$ & $289(6)$ \\
& & & & & \\
Pentadekan & $86.6(9)$ & $0.250(20)$ & $15.0(15)$ & & \\
& $43(19)$ & $0.320(40)$ & $9.1(21)$ & $0.03(\mathrm{f})$ & $261(111)$ \\
& & & & & \\
Hexadekan & $91.9(5)$ & $0.290(10)$ & $16.8(7)$ & & \\
& $52(17)$ & $0.337(24)$ & $12.6(17)$ & $0.03(\mathrm{f})$ & $237(99)$
\end{tabular}

Tabelle 4.5: Ergebnisse aus der Regression dreier Alkan-Spektren mit ein und zwei DebyeSpektralfunktionen. Parameter aus der Ein-Debye-Darstellung nach [51]. Alle Werte für $25^{\circ} \mathrm{C}$. $B: B$-Wert nach Gl. (3.10), $\tau_{\mathrm{D}_{i}}$ : Relaxationszeit der $i$-ten Einzelzeitrelaxation, $A_{\mathrm{D}_{i}}$ : Relaxationsamplitude der $i$-ten Einzelzeitrelaxation, (f) : Parameter wurde bei der Anpassung festgehalten, Nummerierung der Prozesse von großen zu kleinen Relaxationszeiten, Prozesse mit ähnlichen Relaxationszeiten und Amplituden sind gemeinsamen Spalten zugeordnet.

die in ihrer Größenordnung mit denen der Oligoethylenglykole vergleichbar sind (Tab. 4.5). Bei Tetradekan musste neben der zweiten Relaxationszeit noch der $B$ Wert festgehalten werden, um ein stabiles Regressionsergebnis zu erhalten. Das ist nicht verwunderlich, da die Relaxationsfrequenz der nächst höheren Mode bei etwa $8 \mathrm{GHz}$ liegen sollte. Es lässt sich also feststellen, dass die Existenz einer weiteren Mode bei Tetradekan durchaus möglich erscheint.

\subsubsection{Vergleich der gemessenen Relaxationszeiten mit den exakten Berechnungen des Modells}

Es fällt auf, dass die eigentlich nur für längere Polymerketten gültige Näherungsgleichung (4.7) des Rouse-Modells die Relaxationszeiten bei den zum Teil sehr kurzen untersuchten Oligoethylenglykolen immer noch beschreibt (s. Abb. 4.13). Nach den exakten Berechnungen (4.6) wäre zu erwarten, dass für Ketten mit weniger als vier Rouse-Segmenten der Relaxationszeitverlauf, wie in Abb. 4.19 gezeigt, von der $z^{2}$-Abhängigkeit abweicht. Bei der ermittelten Segmentlänge von etwa 3.3 rotationsfähigen Gruppen, sollte also zumindest für Ethylen- und Triethylenglykol eine größere Relaxationszeit beobachtet werden, als von der Näherungsgleichung vorausgesagt.

Eine Erklärung dafür, dass die Relaxationszeit bei kurzen Ketten kleiner als erwartet ausfällt, könnte eine Kettenlängenabhängigkeit der Reibungskonstante $\zeta$ sein, die erst bei kurzen Ketten an Bedeutung gewinnt und daher in dem für lange Ketten entwickelten Rouse-Modell nicht enthalten ist. In diesem Modell berücksichtigt die Reibungskonstante die Wechselwirkung eines einzelnen Segments mit seiner unmittelbaren Umgebung. Diese wird für mittlere und lange Ketten nahezu unabhängig von der Segmentanzahl der Polymere sein, da die Verschiebung eines 


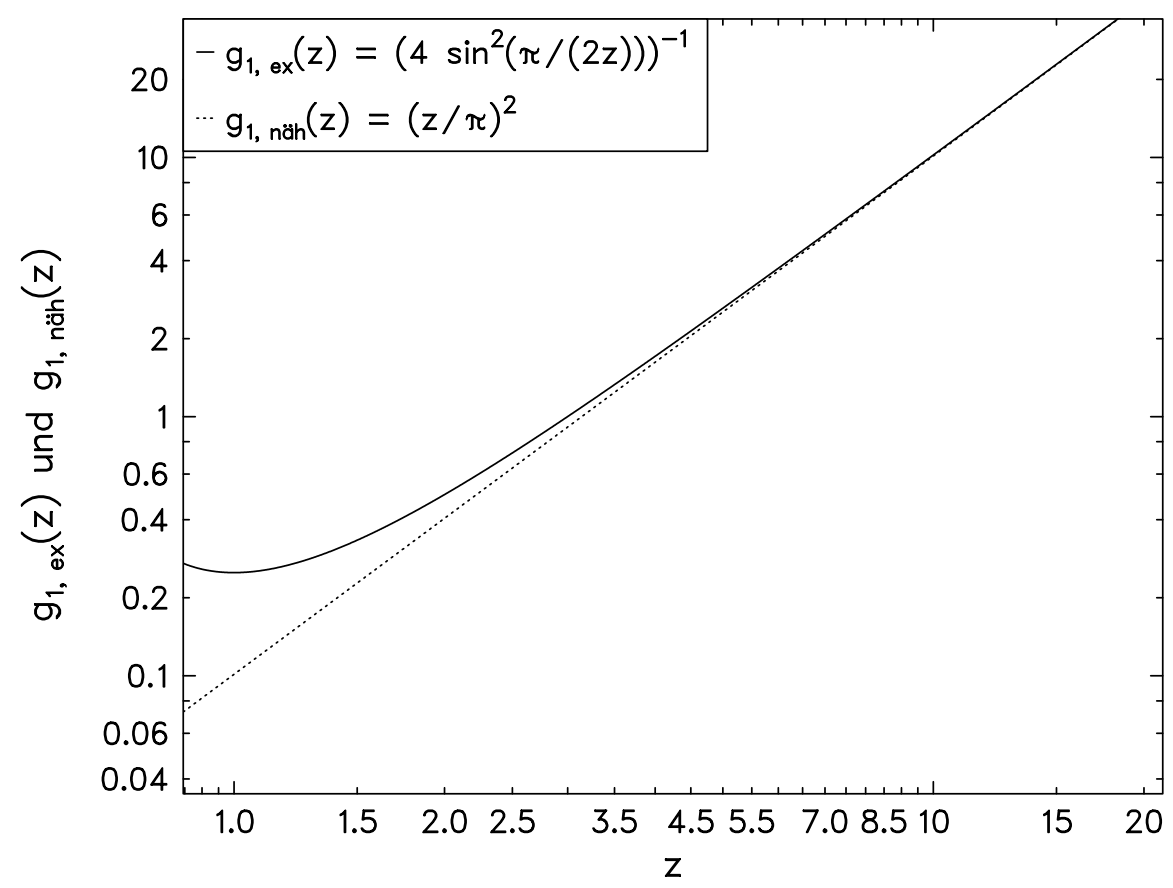

Abbildung 4.19: Zum Vergleich der exakten und der genäherten Gleichung für die erste RouseRelaxationszeit $\tau_{1}$ als Funktion der Segmentanzahl $z$. Aufgetragen sind die Funktionen $g_{1, \mathrm{ex}}(z):=$ $(D / \zeta) \cdot \tau_{1, \text { ex }}(z)=$, wobei $\tau_{1 \text {,ex }}$ die nach Gl. (4.6) exakt berechnete Relaxationszeit bezeichnet sowie $g_{1, \text { näh }}(z):=(D / \zeta) \cdot \tau_{1, \text { näh }}(z)$ mit der aus der Näherungsgleichung (4.7) folgenden Relaxationszeit $\tau_{1, \text { näh }}$.

Segments bei der Modenbewegung klein gegenüber der Polymerlänge sein wird. Das Segment wird also immer etwa die gleiche Umgebung „,sehen”. Dies ändert sich aber, wenn die Kettenlänge in die Größenordnung eines Segments kommt: Da die Verschiebung eines Segments gegen viele kleine umliegende Moleküle leichter ist als gegen wenige große, sollte die Reibungskonstante und damit auch die Relaxationszeit mit abnehmender Kettenlänge kleiner werden. Dies deckt sich mit den Beobachtungen.

Ein weiterer Grund für die Abweichung von der Rouseschen Vorhersage könnte sein, dass die Modellierung des Polymers durch ein diskretes Masse-Feder-System für kurze Ketten nicht mehr gut genug ist. Die Zuordnung von Federn und Massen zu Polymerabschnitten wird am Kettenende schwierig, da es zwar sinnvoll erscheint, die Schwerpunkte der Segmente als Massen aufzufassen, es aber ebenso sinnvoll ist, auch die Kettenenden mit Massen zu versehen, weil dort noch Kräfte angreifen können. Beides ist nicht gleichzeitig möglich. Während dieser Diskretisierungsfehler für lange Ketten nicht ins Gewicht fällt, könnte er für kurze Ketten wesentlich sein. 


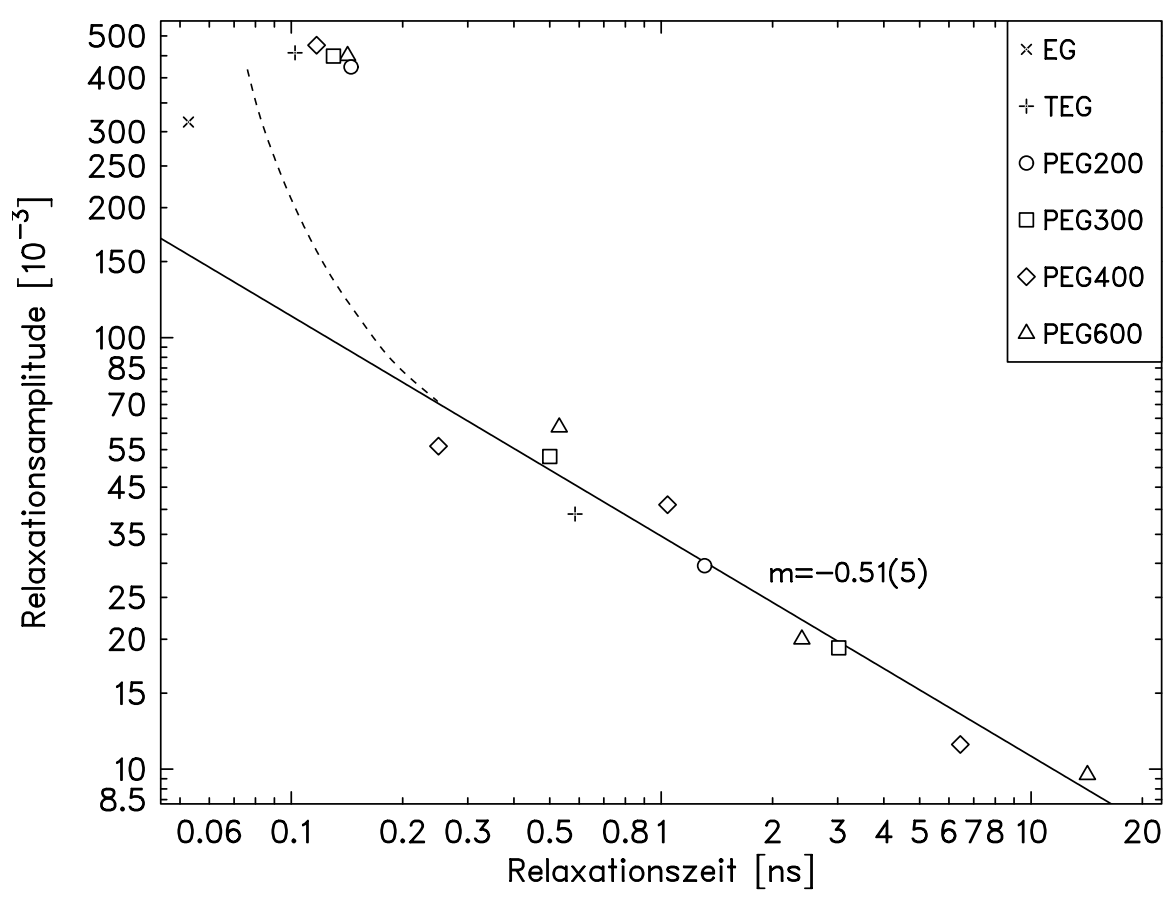

Abbildung 4.20: Relaxationsamplituden als Funktion der zugehörigen Relaxationszeit für die untersuchten Oligoethylenglykole. Alle Werte für $25^{\circ} \mathrm{C}$ nach Tabelle 4.3. Durchgezogene Linie: Regression ohne Amplituden > 0.1, die gestrichelte Linie dient nur als Hilfslinie.

\subsubsection{Die Amplituden der Rouse-Moden}

In den vorangegangenen Abschnitten wurden die beobachteten Absorptionsprozesse anhand ihrer Relaxationszeiten den ersten ungeraden Rouse-Moden der Polymerkettenbewegung zugeordnet. Betrachtet man die Relaxationsamplituden dieser Moden, so ist eine Eigenschaft besonders bemerkenswert: Unabhängig von Kettenlänge oder Modenordnung ist mit zunehmender Relaxationszeit $\tau$ eine Abnahme der Relaxationsamplitude $A$ zu beobachten. Für Relaxationszeiten oberhalb von 0.2 ns gilt dabei ungefähr $A \sim \tau^{-0.5}$ (Abb.4.20). Zu kleineren Relaxationszeiten hin ist dagegen für alle PEG-Spektren eine deutliche Abweichung von diesem Verhalten zu beobachten. Da die entsprechende Relaxationsfrequenzen alle in den oberen Rand des Messbereichs fallen (>0.8 GHz), liegt es zunächst nahe, Messfehler oder systematische Fehler bei der Regressionsanalyse als Ursache für die Abweichung zu vermuten. Ein reiner Messfehler in dieser Größenordnung ist jedoch auszuschließen, wie in Abb.4.21 verdeutlicht wird. Eine erneute Analyse der Daten zeigt außerdem, dass weder durch eine Variation des unsicheren $B$-Werts noch durch die Einführung zusätzlicher hochfrequenter Einzelzeitrelaxationen eine ausreichend große und für alle Kettenlängen konsistente Verringerung der Amplituden hervorgerufen werden kann. Abb. 4.21 zeigt ein Beispiel für solch eine misslungene Anpassung. Es ist daher anzunehmen, dass es sich bei dem verstärkten Anstieg der Amplituden für hochfrequente Moden um einen tatsächlich vorhandenen Effekt handelt. Wegen der geringen Messpunktanzahl kann jedoch keine sichere Aussage über den Verlauf 

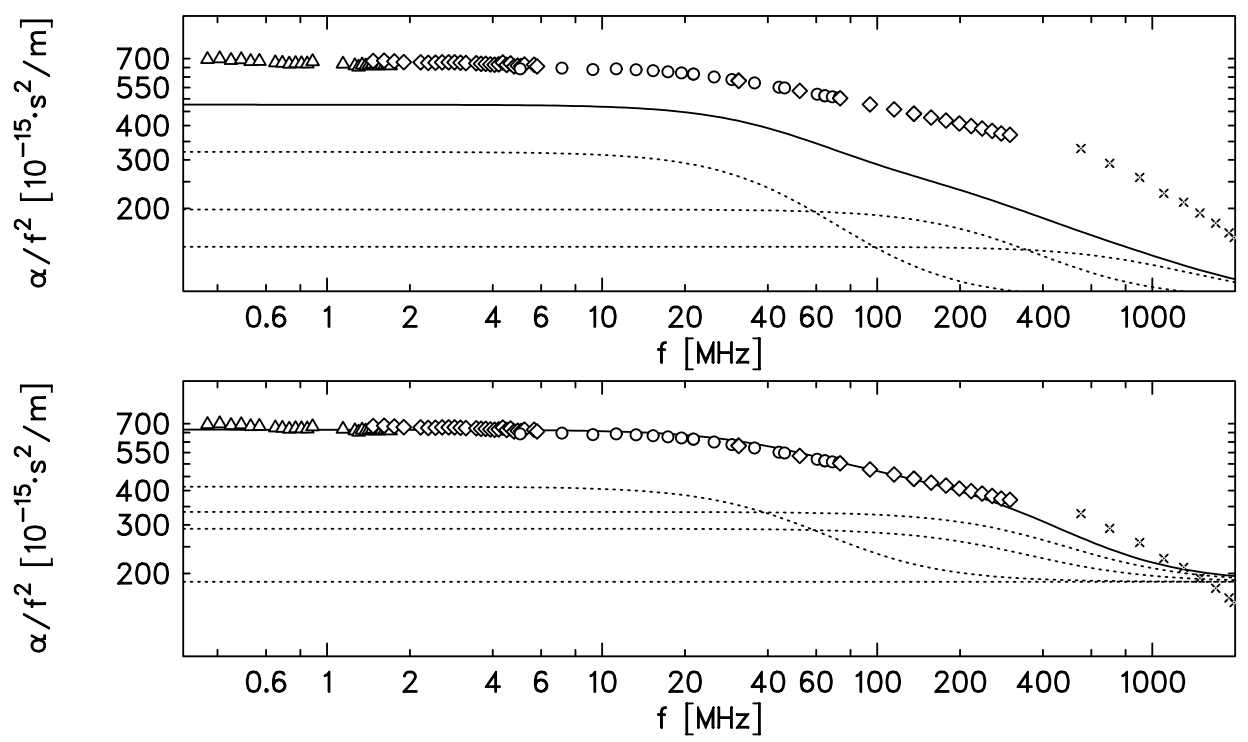

Abbildung 4.21: Ergebnisse einer wiederholten Regressionsanalyse zur Abschätzung der Fehler in den Amplituden der hochfrequenten Moden am Beispiel PEG 300. Oben: Die durchgezogenen Linie beschreibt die resultierende Summe der Einzelzeitrelaxationen (gestrichelte Linien), wenn alle Anpassungsparameter wie in 4.2 beibehalten werden und nur die Amplitude der höchsten Mode auf 0.1 abgesenkt wird. Unten: Regression mit $\tau_{\mathrm{D} 2}, \tau_{\mathrm{D} 3}, A_{\mathrm{D} 2}, A_{\mathrm{D} 3}$ wie in $4.3, A_{\mathrm{D} 4}=0.1$ (fest) und freiem $\tau_{\mathrm{D} 4}$ bzw. $B$.

in diesem Bereich gemacht werden, so dass bei der folgenden Diskussion zunächst nur Moden mit Relaxationszeiten über $0.2 \mathrm{~ns}$ berücksichtigt werden.

Aufgrund der Korrelation von Amplitude und Relaxationszeit sollte aus der in den vorangegangenen Abschnitten diskutierten Abhängigkeit der Relaxationszeit von Kettenlänge und Modenordnung auch eine Abhängigkeit der Amplituden von diesen Parametern folgen. Wegen $\tau \sim z^{2.4} p^{-2}$ und $A \sim \tau^{-0.5}$ wäre demnach die Beziehung

$$
A \sim z^{-1.2} p^{1.1}
$$

zu erwarten. In Abb.4.22 sind die Relaxationsamplituden als Funktion der Kettenlänge für die ersten beiden Moden dargestellt. Auch wenn für die zweite Mode das Regressionsergebnis wegen der geringen Zahl der Datenpunkte nur als grobe Tendenz angesehen werden kann, liegen die Steigungen in der doppeltlogarithmischen Auftragung mit $m(p=1)=-1.058(104)$ und $m(p=2)=-1.419(252)$ doch gut im Bereich des erwarteten Wertes. Das gleiche gilt für den Zusammenhang von Relaxationsamplitude und Modenordnung: Im doppeltlogarithmischen Masterplot $\left(A z^{1.06}\right)(p)$ ergibt sich die Steigung $m=1.003(86)$ (Abb.4.23). Geht man stattdessen von einer $z^{-1.42}$-Abhängigkeit der Amplituden aus, erhält man entsprechend $A z^{1.42} \sim p^{1.120(116)}$

Die Schwierigkeit bei der Interpretation dieser in sich erfreulich konsistenten Ergebnisse ist, dass keine geschlossene Theorie bekannt ist, die Vorhersagen für die Amplituden der Rouse-Moden im Ultraschallabsorptionsspektrum macht. Ein Versuch, die gemessenen Amplituden durch den üblicherweise bei durch Einzelzeitre- 


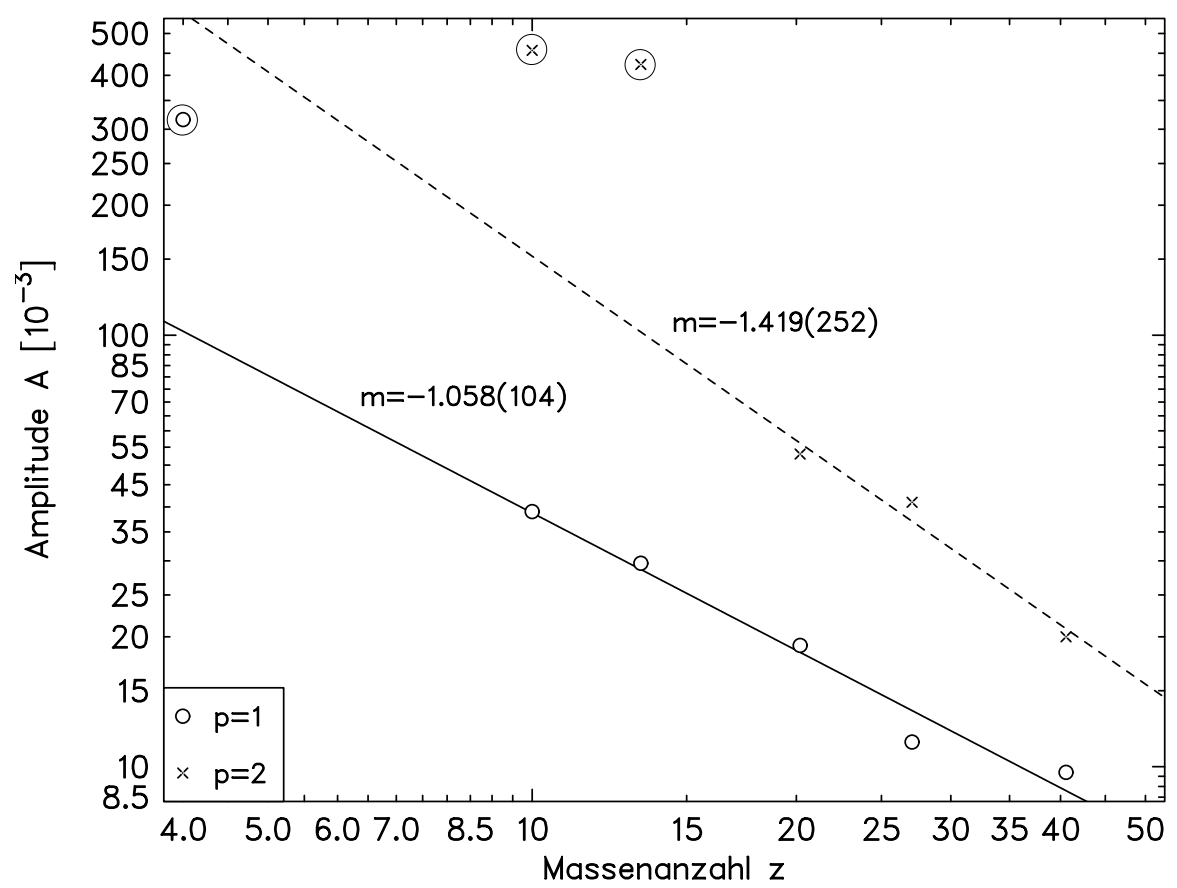

Abbildung 4.22: Relaxationsamplituden als Funktion der Kettenlänge für die untersuchten Oligoethylenglykole. $z$ entspricht wie zuvor der Anzahl der Kohlenstoff- und Sauerstoffatome in den Ketten. Alle Werte für $25^{\circ} \mathrm{C}$ nach Tabelle 4.3. Eingekreiste Punkte gehören zu Moden mit $\tau$ $<0.2 \mathrm{~ns}$ und wurden bei der Regression nicht berücksichtigt.

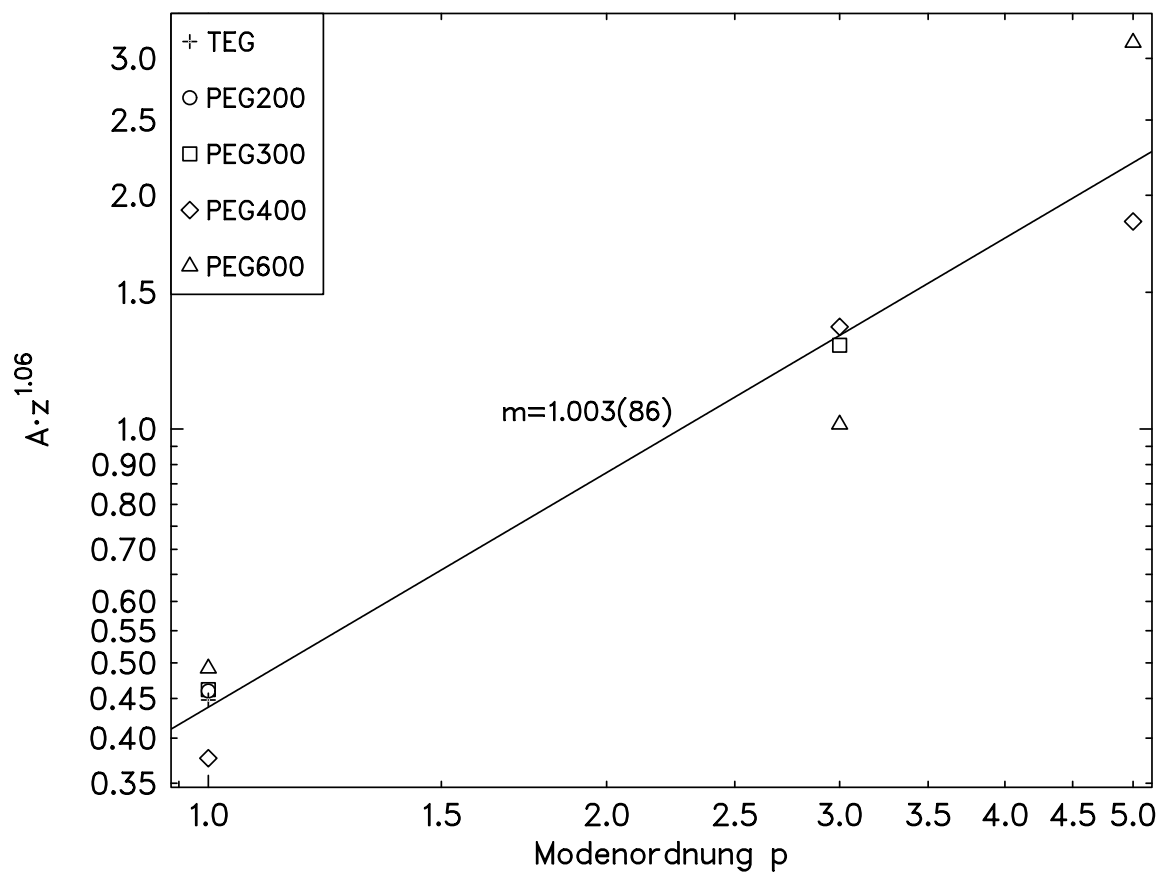

Abbildung 4.23: Zusammenhang von Relaxationsamplituden und Modenordnung. Masterplot zur Berücksichtigung der Kettenlängenabhängigkeit $A \sim z^{1.06}$. Moden mit Relaxationszeiten oberhalb von $0.2 \mathrm{~ns}$ wurden nicht berücksichtigt. 
laxationen beschriebenen monomolekularen Reaktionen verwendeten Ausdruck

$$
A_{\mathrm{D}}=\frac{\pi c_{\mathrm{s}}^{2} \rho}{R T} \frac{K c}{(K+1)^{2}}\left(\frac{\theta}{c_{\mathrm{p}} \rho} \Delta H-\Delta V\right)^{2}
$$

aus der Theorie der chemischen Relaxation [54, 55] zu erfassen, in den neben dem adiabatischen Reaktionsvolumen $\Delta V$ und der Reaktionsenthalpie $\Delta H$, der Gaskonstante $R$ und der Temperatur $T$ die Gleichgewichtskonstante $K$, die Gesamtkonzentration $c$, die spezifische Wärmekapazität $c_{\mathrm{p}}$, der thermische Ausdehnungskoeffizient $\theta$ sowie Dichte und Schallgeschwindigkeit der Flüssigkeit eingehen, wurde verworfen, da er das temperaturabhängige Verhalten der Regressionsparameter nicht richtig wiedergibt.

Rouse selbst gibt in seinem in [6] vorgestellten Modell nur Amplituden für die Scherviskositätsrelaxation an; die Volumenviskosität, die mit in den Absorptionskoeffizienten einfließt (Gl. (3.4)), bleibt dabei unberücksichtigt. Geht man zunächst davon aus, dass der Anteil der Volumenviskosität am Absorptionskoeffizienten gegenüber dem Scherviskositätsanteil vernachlässigbar ist, so kann aus dem RouseModell ein Ausdruck für die Relaxationsamplituden einzelner Moden im Absorptionsspektrum gewonnen werden: Für den Verlustanteil $\eta_{\mathrm{s}}$ der komplexen Scherviskosität $\eta_{\mathrm{s}}^{*}$ erhält Rouse den Ausdruck

$$
\eta_{\mathrm{s}}(\omega)=\eta_{\mathrm{solv}}+n k T \sum_{p=1}^{z-1} \frac{\tau_{p}}{1+\left(\omega \tau_{p}\right)^{2}}
$$

wobei $\tau_{p}$ die Relaxationszeit der $p$-ten Mode, $k$ die Boltzmannkonstante, $T$ die absolute Temperatur, $n$ die Teilchenanzahldichte und $\eta_{\text {solv }}$ die Viskosität des Lösungsmittels bezeichnet. Unter Vernachlässigung der Volumenviskosität ergibt sich durch Einsetzen von Gl. (4.11) in Gl. (3.4)

$$
\frac{\alpha}{f^{2}}=\frac{8 \pi^{2}}{3 \rho c_{\mathrm{s}}^{3}}\left(\eta_{\mathrm{solv}}(\omega)+n k T \sum_{p=1}^{z-1} \frac{\tau_{p}}{1+\left(\omega \tau_{p}\right)^{2}}\right)
$$

Durch Koeffizientenvergleich mit Gl. (3.9) folgt dann für die Relaxationamplitude der $p$-ten Mode im Ultraschallabsorptionsspektrum

$$
A_{p}=\frac{4 \pi}{3 \rho c_{\mathrm{s}}^{2}} n k T
$$

Dabei wurde die Dispersion der Schallgeschwindigkeit vernachlässigt. Die Viskosität des Lösungsmittels wurde für die untersuchten Schmelzen als der Anteil der Scherviskosität $\eta_{\mathrm{s}}$ aufgefasst, der nicht durch die Modendynamik hervorgerufen wird und oberhalb des betrachteten Frequenzbereichs relaxiert. So interpretiert, kann der Summand $\eta_{\text {solv }} \cdot 8 \pi^{2} /\left(3 \rho c_{\mathrm{s}}^{3}\right)$ mit dem Hintergrundsanteil $B / c_{\mathrm{s}}$ gleichgesetzt werden. Die Anzahldichte der Oligomerketten in der Schmelze kann aus ihrer Dichte und ihrer Molmasse bestimmt werden. Dichte und Schallgeschwindigkeit 


\begin{tabular}{l|c|c} 
Oligomer & $\begin{array}{c}\text { aus Rouse-Modell berechnete } \\
\text { Relaxationsamplitude }\left[10^{-3}\right]\end{array}$ & $\begin{array}{c}\text { kleinste experimentell ermittelte } \\
\text { Amplitude (1. Mode) }\left[10^{-3}\right]\end{array}$ \\
\hline \hline EG & 60.9 & 316 \\
TEG & 26.6 & 39.0 \\
PEG 200 & 20.2 & 29.6 \\
PEG 300 & 13.5 & 19.1 \\
PEG 400 (II) & 10.2 & 11.4 \\
PEG 600 & 6.9 & 9.7 \\
& & 6.9
\end{tabular}

Tabelle 4.6: Gegenüberstellung der jeweils kleinsten gemessenen (vergl. Tab.4.3) und der aus dem Rouse-Modell nach Gl. (4.13) berechneten Relaxationsamplitude für verschiedene Oligomere.

wurden für alle Schmelzen gemessen, so dass sich die Relaxationsamplitude direkt berechnen lässt. Das Ergebnis ist in Tab.4.6 bzw. in Abb.4.24 im Vergleich zur jeweils kleinsten experimentell ermittelten Amplitude (1. Mode) für verschiedene Kettenlängen dargestellt.

Die Abnahme der Amplituden mit der Kettenlänge folgt in beiden Datensätzen nahezu demselben Potenzgesetz (Abb. 4.24). Allerdings liegt der Absolutwert der nach Gl. (4.13) bestimmten Amplituden immer unter den experimentell ermittelten Werten. Besonders auffällig ist jedoch, dass jene unabhängig von der Modenordnung sind. Dies widerspricht den experimentellen Beobachtungen, wonach die Amplitude zu höheren Moden hin anwächst. Mit zunehmender Modenordnung wird also die Differenz zwischen den aus den Messungen ermittelten und den berechneten Werten immer größer.

Diese Unterschiede sprechen dafür, dass neben der Scherviskosität auch die im Rouse-Modell nicht berücksichtigte Volumenviskosität merklich zur Relaxationsamplitude der Moden im Schallabsorptionsspektrum beiträgt. Ihr Beitrag erhöht sich offenbar noch mit zunehmender Modenordnung. Dass aber die Volumenviskosität nicht allein als Erklärung für die Abweichungen ausreicht, lassen folgende Ergebnisse vermuten: Vorausgesetzt, dass unterhalb des untersuchten Frequenzbereichs keine weiteren Relaxationen in den Oligomerspektren mehr auftreten, kann der Scherviskositätsanteil am gemessenen tieffrequenten $\alpha / f^{2}$-Wert anhand von Gl. (3.4) durch

$$
\left(\frac{\alpha}{f^{2}}\right)_{\text {tieffreq, }, \eta_{\mathrm{s}}(0)}=\frac{8 \pi^{2}}{3 \rho c_{\mathrm{s}}^{3}} \eta_{\mathrm{s}}(\omega \rightarrow 0)
$$

aus der statischen Scherviskosität $\eta_{\mathbf{s}}(\omega \rightarrow 0)$ abgeschätzt werden. Andererseits lässt sich der tieffrequente Scherviskositätsanteil mit Hilfe der „Rouse-Amplituden“ $A_{p}$ 


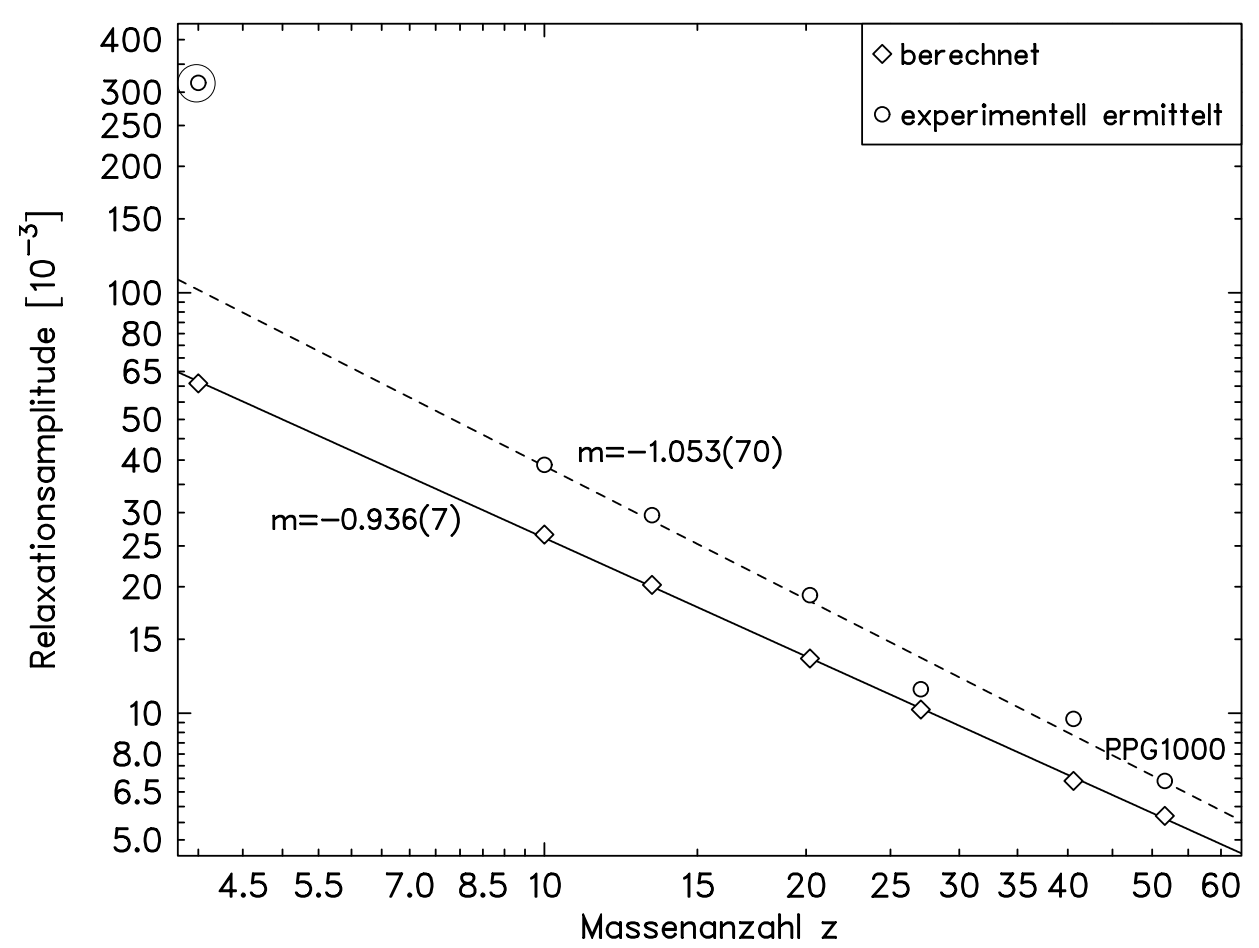

Abbildung 4.24: Kettenlängenabhängigkeit der kleinsten experimentell ermittelten (Tab.4.3) und der nach Gl. (4.13) berechneten Amplituden für die Oligomere aus Tab.4.6. Die Massenzahl $z$ entspricht der Anzahl der Kohlenstoff- bzw. Sauerstoffatome im Polymerrückgrat. Durchgezogene Linie: Regression für die berechneten Amplituden unter Einbeziehung des Werts für PPG 1000. Gestrichelte Linie: Regression der Messergebnisse für $\tau>0.2 \mathrm{~ns}$, also ohne den Wert für Ethylenglykol (eingekreist). 


\begin{tabular}{l|c|c|c} 
Oligomer & $\begin{array}{c}\left(\frac{\alpha}{f^{2}}\right)_{\text {tieffreq,mess }} \\
{\left[10^{\left.-15 \frac{\mathrm{s}^{2}}{\mathrm{~m}}\right]}\right.}\end{array}$ & $\begin{array}{c}\left(\frac{\alpha}{f^{2}}\right)_{\text {tieffreq, } \eta_{\mathrm{s}}(0)} \\
{\left[10^{-15} \frac{\mathrm{s}^{2}}{\mathrm{~m}}\right]}\end{array}$ & $\begin{array}{c}\left(\frac{\alpha}{f^{2}}\right)_{\text {tieffreq,Rouse }} \\
{\left[10^{\left.-15 \frac{\mathrm{s}^{2}}{\mathrm{~m}}\right]}\right.}\end{array}$ \\
\hline \hline EG & 155 & 89 & 103 \\
TEG & 370 & 211 & 164 \\
PEG 200 & 560 & 290 & 218 \\
PEG 300 & 670 & 406 & 288 \\
PEG 400 (II) & 820 & 540 & 410 \\
PEG 600 & 1220 & 785 & 563 \\
\hline PPG 1000 & 2450 & 1580 & 951
\end{tabular}

Tabelle 4.7: Gemessener tieffrequenter $\alpha / f^{2}$-Wert (bei $1.5 \mathrm{MHz}$ ) sowie nach Gl. (4.14) und Gl. (4.15) berechneter Scherviskositätsanteil am gemessenen $\alpha / f^{2}$-Wert für verschiedene Oligomere. Alle Werte für $25^{\circ} \mathrm{C}$.

und der im Limes $\omega \rightarrow 0$ betrachteten Gl. (3.9)

$$
\left(\frac{\alpha}{f^{2}}\right)_{\text {tieffreq, Rouse }}=\frac{2 \pi}{c_{\mathrm{s}}} \sum_{p=1}^{z-1} A_{p} \tau_{p}+\frac{B}{c_{\mathrm{s}}}
$$

mit den experimentell ermittelten Relaxationszeiten $\tau_{p}$, B-Werten und Schallgeschwindigkeiten $c_{\mathrm{s}}$ bestimmen. Tabelle 4.7 zeigt eine Gegenüberstellung der auf beide Arten ermittelten tieffrequenten scherviskosen Anteile. Außerdem ist der tieffrequente, d.h. bei $1.5 \mathrm{MHz}$ gemessene, $\alpha / f^{2}$-Wert für die verschiedenen Schmelzen angegeben.

Außer für Ethylenglykol bleiben die aus den „Rouse-Amplituden“ folgenden tieffrequenten $\alpha / f^{2}$-Werte unter dem nach Gl. (4.14) erwarteten Scherviskositätsanteil. Ein möglicher Grund für diese Unterschiede könnten weitere tieffrequente Relaxationsprozesse sein, die zusätzlich zu den Rouse-Moden zum tieffrequenten scherviskosen Anteil $\left(\alpha / f^{2}\right)_{\text {tieffreq, } \eta_{\mathbf{s}}(0)}$ beitragen, im untersuchten Spektralbereich aber nicht detektiert werden können. Eine andere mögliche Ursache ist die unzureichende Beschreibung der Scherviskositätsanteile durch das Rouse-Modell. Hier könnten erst tieffrequentere Messungen der Ultraschallabsorption oder der Scherviskosität weiteren Aufschluss geben. Ungeklärt bleibt auch die molekulare Ursache für die Korrelation von Relaxationszeit und Amplituden, die, wie auch die Modenabhängigkeit der Amplituden, nicht durch den aus dem Rouse-Modell gewonnenen Ausdruck (4.13) erklärt werden kann. 


\begin{tabular}{l|c|c|c} 
Substanz & $\begin{array}{c}\text { statische Scherviskosität } \eta_{\mathrm{s}} \\
\text { (gemessen) [mPas] }\end{array}$ & $\begin{array}{c}\text { Volumenviskosität } \eta_{\mathrm{v}} \\
\text { nach Gl. (4.16) [mPas] }]\end{array}$ & $\eta_{\mathrm{v}} / \eta_{\mathrm{s}}$ \\
\hline \hline & 17.1 & 16.7 & 0.98 \\
EG & 37.5 & 37.6 & 0.99 \\
TEG & 50.7 & 62.9 & $1.24^{1)}$ \\
PEG 200 & 70.4 & 61.0 & 0.87 \\
PEG 300 & 93.1 & 64.2 & 0.69 \\
PEG 400 (II) & 133.5 & 98.8 & 0.74 \\
PEG 600 & 149.6 & 109.0 & 0.73 \\
PPG 1000 & & &
\end{tabular}

Tabelle 4.8: Verhältnis von Volumen- und Scherviskosität bei tiefen Frequenzen. Alle Werte für $25^{\circ} \mathrm{C}$. 1) : enthält einen auf Verunreinigungen zurückgeführten zusätzlichen tieffrequenten Prozess (Abschnitt4.2.3.1).

\subsubsection{Verhältnis von Scher- und Volumenviskosität bei tiefen Frequenzen}

Das Verhältnis von Volumen- und Scherviskosität in polymeren Flüssigkeiten ist in der Vergangenheit vielfach diskutiert worden [56-59]. Verschiedene theoretische Ansätze [58, 60] sagen einen konstanten Wert von $\eta_{\mathrm{s}} / \eta_{\mathrm{v}}=2 / 3$ voraus. Frequenzabhängige Messungen des Schallabsorptionskoeffizienten und der Scherviskosität von Polymerschmelzen und -lösungen bestätigen zwar die erwartete Frequenzund Temperaturunabhängigkeit von $\eta_{\mathrm{v}} / \eta_{\mathrm{s}}$, ergeben jedoch einen merklich höheren Wert, der je nach Flüssigkeit, Konzentration und Lösungsmittel zwischen 1.5 und 5 schwankt [56, 58, 61].

Zwar wurden für die in dieser Arbeit untersuchten PEG- und PPG-Schmelzen keine breitbandigen frequenzabhängigen Messungen der Scherviskosität vorgenommen, dennoch kann aus den gemessenen statischen Viskositäten und tieffrequenten $\alpha / f^{2}$-Werten zumindest das Verhältnis von Volumen- und Scherviskosität bei kleinen Frequenzen abgeschätzt werden. Aus Gl. (3.4) folgt:

$$
\eta_{\mathrm{v}} \approx \frac{\rho c_{\mathrm{s}}^{3}}{2 \pi^{2}} \cdot\left(\left(\frac{\alpha}{f^{2}}\right)_{\text {tieffreq,mess }}-\left(\frac{\alpha}{f^{2}}\right)_{\text {tieffreq, } \eta_{\mathrm{s}}(0)}\right)
$$

Mit den entsprechenden Werten aus Tab. 4.7 erhält man die in Tabelle 4.8 aufgelisteten Ergebnisse.

Bei den längeren Oligomere liegt $\eta_{\mathrm{v}} / \eta_{\mathrm{s}}$ nahe bei dem von der Theorie erwarteten Wert. Für die kürzeren Ketten nimmt das Verhältnis zwar etwas zu, bleibt aber unter 1. Einzige Ausnahme stellt PEG 200 dar. Der höhere Wert wird darauf zurückgeführt, dass in dem betreffenden Spektrum ein zusätzlicher tieffrequenter Prozess auftritt. Dieser vermutlich durch Verunreinigungen verursachte Prozess sorgt für einen entsprechend höheren tieffrequenten $\alpha / f^{2}$-Wert und erhöht so 
scheinbar den Volumenviskositätsanteil. Zieht man den Beitrag dieses Zusatzterms von $\left(\alpha / f^{2}\right)_{\text {tieffreq,mess }}$ ab, sinkt $\eta_{\mathrm{v}} / \eta_{\mathrm{s}}$ auf 0.95 und fügt sich sehr gut in die Reihe ein. Insgesamt stimmen diese Ergebnisse damit besser mit den theoretischen Vorhersagen überein als die Werte von Alig et al. [56, 58, 61], zeigen aber eine leichte Kettenlängenabhängigkeit.

\subsubsection{Temperaturabhängigkeit von Relaxationszeit und -amplitude}

Für PEG 400 (I) wurden Messungen bei drei verschiedenen Temperaturen, $10^{\circ} \mathrm{C}$, $25^{\circ} \mathrm{C}$ und $40^{\circ} \mathrm{C}$, vorgenommen. Wie auch bei der $25^{\circ} \mathrm{C}$-Messung konnte das Absorptionsverhalten bei den beiden anderen Temperaturen durch vier Debye-Spektralfunktionen beschrieben werden, wobei die tieffrequente Einzelzeitrelaxation auf Verunreinigungen zurückgeführt wird (Abschnitt 4.2.3.1). Die Funktionsparameter dieser Vier-Debye-Darstellung sind in Tabelle 4.9 aufgelistet.

Die Auswertung der Daten aller übrigen PEG-Schmelzen hat jedoch ergeben, dass dem PEG 400 (I) Spektrum eigentlich fünf Einzelzeitrelaxationen zugrunde liegen sollten: Eine, die den tieffrequenten Zusatzprozess widerspiegelt und vier weitere, die als die ersten vier ungeraden Rouse-Moden interpretiert werden können. Da eine Anpassung mit insgesamt fünf Debye-Funktionen jedoch auch hier zu einer Überbestimmung der Spektren führen würde, wurde wie zuvor auf die Einführung eines weiteren Debye-Terms verzichtet. Insbesondere bei der höchsten Mode sind daher größere systematische Fehler zu erwarten. Sie werden aber bei allen drei Temperaturen ähnlich groß sein, so dass ein sinnvoller Vergleich der Daten dennoch möglich ist.

Lässt man den Zusatzterm (Index D1) unberücksichtigt, zeigt sich folgendes Bild: Die Relaxationzeiten aller beobachteten Prozesse nehmen mit steigender Temperatur leicht ab. Dies steht im Einklang mit dem DTO-Modell [7], da die Energiebarrieren, die verschiedene Konformationen eines Polymers voneinander trennen, bei höherer thermischer Energie des Gesamtsystems mit einer größeren, durch die Boltzmann-Verteilung gegebenen Wahrscheinlichkeit überwunden werden können. Nach einer äußeren Störung wird sich daher das dynamische Gleichgewicht zwischen den Zuständen entsprechend schneller wieder einstellen, was sich in kleineren Relaxationszeiten äußert.

Zusammen mit den Relaxationszeiten nehmen auch die Relaxationsamplituden der Moden mit steigender Temperatur ab. Die in den vorangegangenen Abschnitten beschriebene Korrelation von Relaxationszeit und Amplitude $e^{5}$ setzt sich also im temperaturabhängigen Verlauf nicht fort. Sie bleibt jedoch, wie auch die Korrelationen zwischen Amplituden und Modenordnung sowie Relaxationszeit und Modenordnung bei vorgegebener Temperatur in ihrer Tendenz erhalten.

Eine Erklärung dieses temperaturabhängigen Verhaltens ist mangels eines grundlegenden molekularen Modells für die Relaxationsamplituden der Rouse-Moden

\footnotetext{
${ }^{5}$ Moden mit hohen Relaxationszeiten haben kleine Amplituden und umgekehrt (vergl. 4.3.2.6)
} 
4 Ultraschallabsorptionsspektren der Oligoethylenglykole

\begin{tabular}{c|ccccccccc}
$\begin{array}{c}T \\
{\left[{ }^{\circ} \mathrm{C}\right]}\end{array}$ & $\begin{array}{c}B \\
{[\mathrm{ps}]}\end{array}$ & $\begin{array}{c}\tau_{\mathrm{D} 1} \\
{[\mathrm{~ns}]}\end{array}$ & $\begin{array}{c}A_{\mathrm{D} 1} \\
{\left[10^{-3}\right]}\end{array}$ & $\begin{array}{c}\tau_{\mathrm{D} 2} \\
{[\mathrm{~ns}]}\end{array}$ & $\begin{array}{c}A_{\mathrm{D} 2} \\
{\left[10^{-3}\right]}\end{array}$ & $\begin{array}{c}\tau_{\mathrm{D} 3} \\
{[\mathrm{~ns}]}\end{array}$ & $\begin{array}{c}A_{\mathrm{D} 3} \\
{\left[10^{-3}\right]}\end{array}$ & $\begin{array}{c}\tau_{\mathrm{D} 4} \\
{[\mathrm{~ns}]}\end{array}$ & $\begin{array}{c}A_{\mathrm{D} 4} \\
{\left[10^{-3}\right]}\end{array}$ \\
\hline \hline & & & & & & & & & \\
10 & $149(17)$ & $203(39)$ & $0.74(1)$ & $11.4(5)$ & $17.5(6)$ & $1.17(5)$ & $93(5)$ & $0.209(10)$ & $685(33)$ \\
25 & $147(12)$ & $142(30)$ & $0.64(6)$ & $6.5(3)$ & $12.4(5)$ & $1.02(5)$ & $43.8(22)$ & $0.136(5)$ & $505(15)$ \\
40 & $137(9)$ & $80(9)$ & $0.67(6)$ & $4.2(4)$ & $8.8(12)$ & $0.97(1)$ & $26.0(17)$ & $0.110(7)$ & $325(28)$
\end{tabular}

Tabelle 4.9: Parameter der Beschreibung der temperaturabhängigen Messung von PEG 400 (I) mit vier Debye-Spektralfunktionen. Verwendet wurden die nicht dispersionskorrigierten, mit Harnstoffreferenz ausgewerteten Datensätze. Die angegebenen Fehler berücksichtigen wieder die Ergebnisse aus der Anpassung der dispersionskorrigierten und, soweit vorhanden, der mit Polymerreferenz ausgewerteten Messdaten. Zusatzterme nach Abschnitt 4.2.3.1 sind kursiv gedruckt. $T$ : Temperatur $B: B$-Wert nach Gl. (3.10), $\tau_{\mathrm{D}_{i}}$ : Relaxationszeit der $i$-ten Einzelzeitrelaxation, $A_{\mathrm{D}_{i}}$ : Relaxationsamplitude der $i$-ten Einzelzeitrelaxation, Nummerierung der Prozesse von großen zu kleinen Relaxationszeiten, Prozesse mit ähnlichen Relaxationszeiten und Amplituden sind gemeinsamen Spalten zugeordnet.

im Ultraschallspektrum schwierig. Eine Abschätzung des Temperaturverlaufs mit Hilfe des üblicherweise für monomolekulare Reaktionen verwendeten Ausdrucks (4.10) ließe unter sinnvollen Annahmen $(\Delta V, \Delta H$ temperaturunabhängig, $\Delta H \approx$ $12 \mathrm{~kJ} / \mathrm{mol}$ ) einen deutlichen Anstieg der Amplituden zu höheren Temperaturen hin erwarten: Ca. 35 \% Zuwachs bei einer Erhöhung der Temperatur von $283.15 \mathrm{~K}$ auf 313.15 K. Dies ist jedoch unvereinbar mit der beobachteten Abnahme der Amplitude von bis zu $70 \%$. Ebenso gegenläufig zum experimentellen Ergebnis verhalten sich die „Rouse-Amplituden“ $A_{p}$ (Gl.(4.13)), mit deren Hilfe zumindest die Temperaturabhängigkeit des scherviskosen Anteils in den gemessenen Amplituden abgeschätzt werden kann: $A_{p}$ erhöht sich im betrachteten Temperaturintervall um etwa $25 \%$. Dieses experimentelle Ergebnis für die Temperaturabhängigkeit der Amplituden verlangt - wie deren Verschwinden für die geraden Moden und ihre Abhängigkeit von Relaxationszeit und Modenordnung - nach einer Erweiterung der Theorie viskoelastischen Verhaltens um die Kopplung von Moden der Polymerkettenbewegung an Schallfelder. 


\section{Dielektrische Spektroskopie}

\subsection{Grundlagen}

Unter dem Einfluss eines elektrischen Wechselfeldes $\boldsymbol{E}(\omega)$ mit Kreisfrequenz $\omega=$ $2 \pi f$ stellt sich in einem dielektrischen Medium eine Polarisation $\boldsymbol{P}(\omega)$ ein, die über die komplexe Dielektrizitätszahl

$$
\epsilon(\omega)=\epsilon^{\prime}(\omega)-i \epsilon^{\prime \prime}(\omega)
$$

mit dem elektrischen Feld verknüpft ist:

$$
\boldsymbol{P}(\omega)=\epsilon_{0}(\epsilon(\omega)-1) \boldsymbol{E}(\omega) .
$$

Hierbei bezeichnet $\epsilon_{0}=8.854 \cdot 10^{-12} \mathrm{Vs} /$ Am die elektrische Feldkonstante.

Zur Polarisierbarkeit des Mediums, die durch $\epsilon(\omega)$ widergespiegelt wird, tragen zwei Mechanismen bei: In unpolaren Substanzen, deren molekulare Ladungsverteilung ohne äußere Einflüsse symmetrisch ist, verursacht das elektrische Feld eine Verschiebung von Elektronen und Atomkernen innerhalb eines Moleküls. In polaren Flüssigkeiten tritt zusätzlich zu dieser Verschiebungspolarisation ein weiterer Effekt auf, der die Polarisierbarkeit des Mediums erhöht: Ohne äußeres elektrisches Feld sind die permanenten Dipolmomente der Moleküle zufällig in alle Richtungen verteilt, da sich ihre Orientierungen durch thermische Aktivierung laufend ändern. Unter dem Einfluss eines elektrischen Feldes richten die Dipole sich im Mittel etwas mehr in Richtung der Feldlinien aus, so dass eine Orientierungspolarisation entsteht.

\subsubsection{Dielektrische Relaxation}

Da Umorientierungsprozesse durch molekulare Wechselwirkungskräfte in der Flüssigkeit beeinflusst sind, stellt sich eine Polarisation nicht unmittelbar, sondern mit einer für das System charakteristischen Verzögerung ein. Bei zu hoher Frequenz kann die Polarisation dem äußeren Feld nicht mehr folgen. Es kommt zu einer Abnahme der Polarisierbarkeit, die sich in einer Dispersion von $\epsilon^{\prime}(\omega)$ ausdrückt. Der Imaginärteil $\epsilon^{\prime \prime}(\omega)$ der komplexen Dielektrizitätszahl beschreibt die mit der Dispersion verbundene Dissipation von elektrischer Energie, die in Folge der zunehmenden Phasenverschiebung zwischen Polarisation und elektrischem Wechselfeld auftritt. 
Die Relaxationsfrequenz der Orientierungspolarisation liegt bei vielen Flüssigkeiten im Bereich von $10 \mathrm{MHz}$ bis $1 \mathrm{GHz}$ [62]. Breitbandige Messungen des Dispersions- und Absorptionsspektrums in diesem Bereich lassen somit Rückschlüsse auf molekulare Umstrukturierungsprozesse in der Flüssigkeit zu. Die Verschiebungspolarisation zeigt dagegen erst oberhalb von $100 \mathrm{GHz}$ Dispersion durch resonanzartige Phänomene und trägt daher nur als frequenzunabhängiger Hintergrundsanteil zu den hier vorliegenden Spektren bei, die den Frequenzbereich von $0.4 \mathrm{MHz}$ bis $3 \mathrm{GHz}$ erfassen.

Cole-Cole-Spektralfunktion Zur Beschreibung der in dieser Arbeit gemessenen dielektrischen Spektren wurde die empirische Cole-Cole-Relaxationsfunktion $[63,64]$

$$
\epsilon(\omega)=\epsilon^{\prime}(\omega)-i \epsilon^{\prime \prime}(\omega)=\epsilon(\infty)+\frac{\epsilon(0)-\epsilon(\infty)}{1+\left(i \omega \tau_{\mathrm{CC}}\right)^{1-h}}
$$

verwendet, der eine kontinuierliche Relaxationszeitverteilung mit Gewichtsfunktion

$$
G(\tau)=\frac{\sin (\pi h)}{2 \pi\left(\cosh \left((1-h) \ln \left(\frac{\tau}{\tau_{\mathrm{CC}}}\right)\right)-\cos (\pi h)\right)}, \quad 0 \leq h<1
$$

zugrunde liegt. In logarithmischer Auftragung verläuft $G(\tau)$ symmetrisch um $\ln \left(\tau / \tau_{\mathrm{CC}}\right)$ und nimmt ihr Maximum bei der Hauptrelaxationszeit $\tau_{\mathrm{CC}}$ an (Abb.5.1). Der Verteilungsparameter $h$ stellt dabei ein Maß für die Breite der Verteilung dar. Für $h \rightarrow 0$ geht die Cole-Cole-Funktion in eine Debye-Spektralfunktion mit diskreter Relaxationszeit über. Mit $\epsilon(0)=\lim _{\omega \rightarrow 0} \epsilon(\omega)$ wird die zu tiefen Frequenzen, mit $\epsilon(\infty)=\lim _{\omega \rightarrow \infty} \epsilon(\omega)$ die zu hohen Frequenzen extrapolierte Dielektrizitätszahl bezeichnet.

\subsubsection{Leitfähigkeit}

Viele Flüssigkeiten enthalten freie Ladungsträger, die zur spezifischen Leitfähigkeit $\sigma$ der Substanz beitragen. Diese führt zu einer Erhöhung des gemessenen negativen Imaginärteils $\epsilon_{\text {mess }}^{\prime \prime}(\omega)$ der Dielektrizitätszahl um den Summanden

$$
\epsilon_{\sigma}^{\prime \prime}(\omega)=\frac{\sigma}{\epsilon_{0} \omega}
$$

der zu höheren Frequenzen hin abnimmt. Um den Relaxationsanteil $\epsilon^{\prime \prime}(\omega)$ vom Leitfähigkeitsanteil zu trennen, wird an die gemessenen Absorptionsspektren eine Summe aus Relaxationsfunktion(en) und einem Term nach Gl. (5.5) mit freiem Parameter $\sigma$ angepasst. 


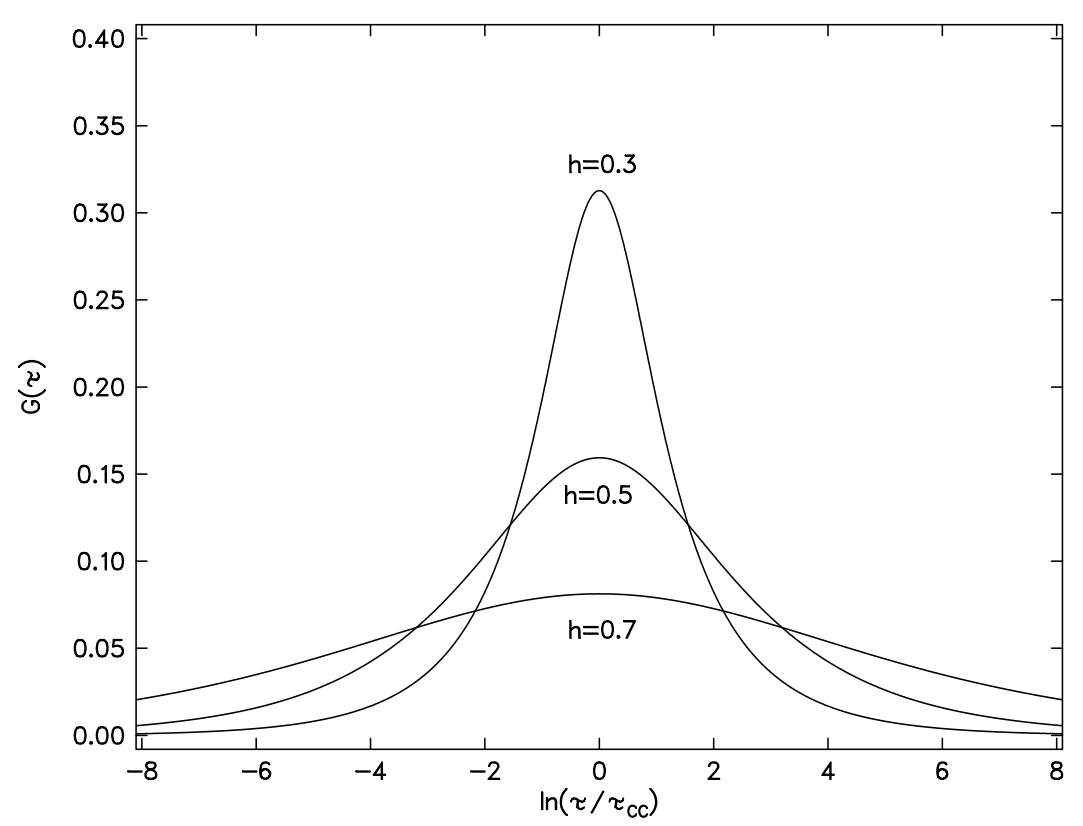

Abbildung 5.1: Relaxationszeitverteilung der Cole-Cole-Spektralfunktion für unterschiedliche Verteilungsparameter $h$.

\subsection{Messverfahren}

\subsubsection{Messprinzip}

Aufbau des Messplatzes Zur Bestimmung der komplexen Dielektrizitätszahl im Frequenzbereich von $0.4 \mathrm{MHz}$ bis $3 \mathrm{GHz}$ wurden mit Hilfe eines computergesteuerten Netzwerkanalysators Reflexionsfaktormessungen an zwei Cut-Off-Messzellen durchgeführt. Abbildung 5.2 zeigt den schematischen Aufbau des Messplatzes.

Das vom Netzwerkanalysator HP 8753 A (NWA, 2) erzeugte Signal wird am Analysatorausgang (A) abgenommen und gelangt in ein Reflexionstestset HP 85044 A (RTS), wo es ein Leistungsteiler (3) in ein Mess- bzw. Referenzsignal gleicher Amplitude aufspaltet. Während das Referenzsignal über den Vergleichseingang (R) direkt an den NWA zurückgegeben wird, wird das Messsignal zur Cut-Off-Zelle (5) weitergeleitet. Das dort reflektierte Signal wird mit Hilfe eines Richtkopplers (4) vom einfallenden Signal getrennt und gelangt an den Messeingang (E) des Netzwerkanalysators. Dieser vergleicht die Signale an den Eingängen (R) und (E) nach Betrag und Phasendifferenz und errechnet daraus den komplexen Reflexionsfaktor. Der Reflexionsfaktor wird bei ca. 50 fest voreingestellten Frequenzen bestimmt und zur weiteren Auswertung an einen Computer (1) übermittelt.

Da zwischen NWA und Messzelle das Reflexionstestset geschaltet ist und diese drei Bauteile über flexible Koaxialleitungen miteinander gekoppelt sind, ist vor jeder Messung eine Kalibrierung des Aufbaus bezüglich der Eingangsebene der Messzelle notwendig. Dazu wird das Reflexionstestset als Sechspol betrachtet, dessen Übertragungseigenschaften durch eine Streumatrix beschrieben werden. Die Para- 


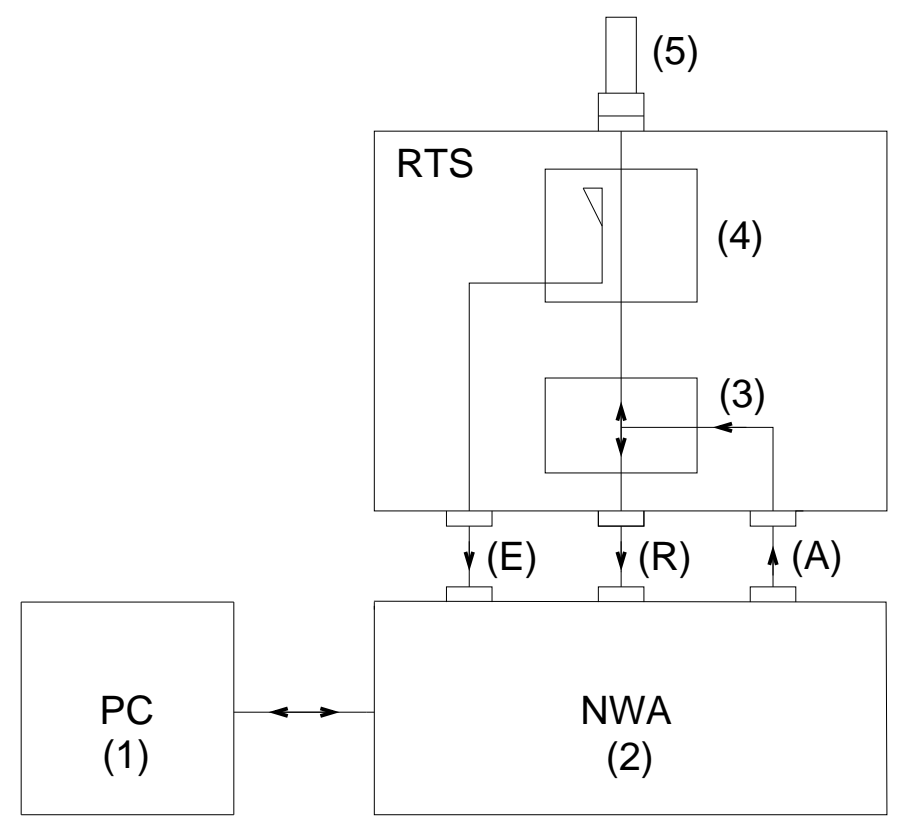

Abbildung 5.2: Schematische Darstellung des Aufbaus zur Messung der Dielektrizitätszahl: (1) Computer zur Steuerung und Datenverarbeitung, (2) Netzwerkanalysator mit Signalausgang (A) und den Eingängen für (R) Referenz- und (E) Messsignal, (3) Leistungsteiler, (4) Richtkoppler, (5) Cut-Off-Messzelle.

meter dieser Matrix werden bei der Kalibrierung ermittelt, indem der Reflexionsfaktor bei drei verschiedenen Abschlüssen (Kurzschluss, offenes Ende, angepasster Abschluss $50 \Omega$ ) bestimmt wird. Genauere Beschreibungen des Kalibriervorgangs und der Parameterbestimmung finden sich in [27, 65-67].

Durch einen Thermostatiermantel, der die Messzelle (5) umschließt, kann die Temperatur während der Messung stabil gehalten werden.

Konstruktion der Messzellen Abbildung 5.3 zeigt den Aufbau einer Cut-OffMesszelle. Sie besteht im Wesentlichen aus einem Koaxial-/Rundhohlleiterübergang. Zur Abdichtung ist in den Koaxialleiter (1, 2) ein Teflonfenster (3) eingelassen, dessen Wellenwiderstand durch geeignete Abmessungen des Fensters an den Wellenwiderstand $Z_{0}=50 \Omega$ der Zuleitung angepasst ist. Oberhalb der Teflondichtung ist die Zelle bis in den Hohlleiterbereich hinein mit der zu untersuchenden Substanz befüllt. Der Hohlleiter wird unterhalb der Grenzfrequenz $\nu_{\mathrm{g}}$ seiner tieffrequentesten Mode betrieben, die im Fall einer verlustfreien Flüssigkeit $\left(\epsilon^{\prime \prime}(\omega)=0\right)$ durch

$$
\nu_{\mathrm{g}}=\frac{2 c_{0}}{2.61 d \sqrt{\epsilon^{\prime}(\omega)}}
$$

gegeben ist [68], wobei $c_{0}$ die Vakuumlichtgeschwindigkeit und $d$ den Innendurchmesser des Hohlleiters bezeichnen. Unterhalb dieser Grenzfrequenz ist keine Wellenausbreitung im Rundhohlleiter möglich; die Amplitude des elektromagnetischen 


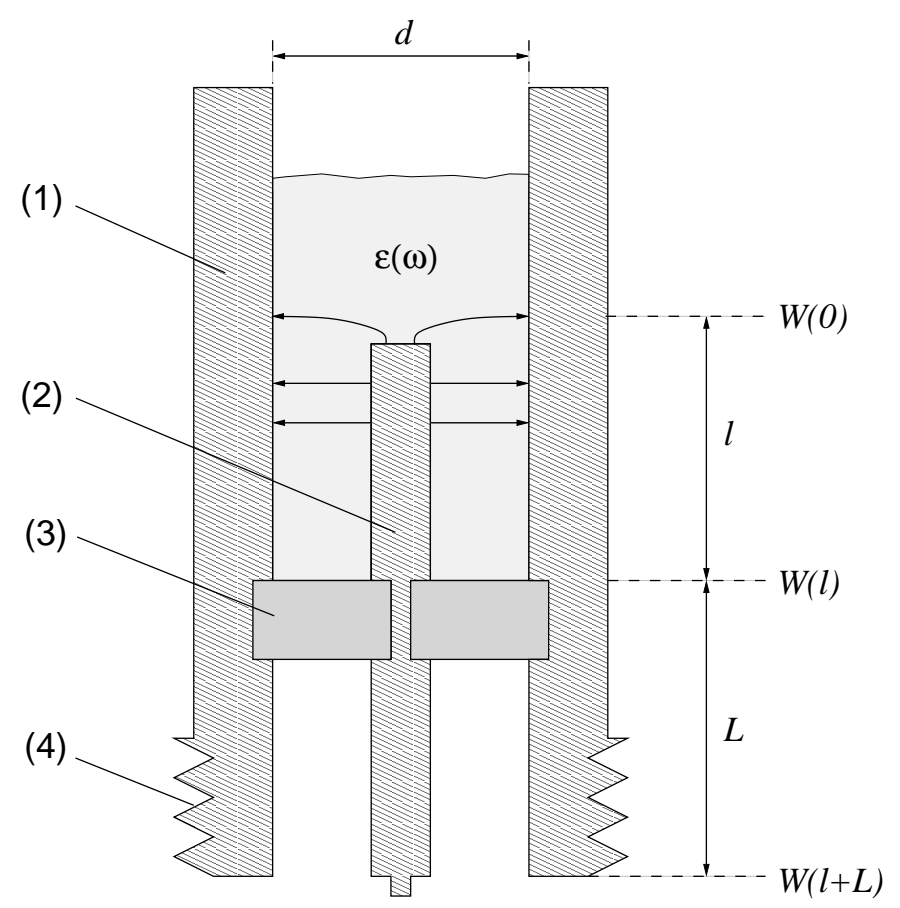

Abbildung 5.3: Schematischer Aufbau einer Cut-Off-Messzelle: (1) Innenleiter, (2) Außenleiter, (3) Teflonfenster, (4) Stecker.

Feldes fällt in diesem Bereich exponentiell ab. Der Übergang in den Hohlleiterbereich kann daher als kapazitiver Abschluss der koaxialen Zuleitung aufgefasst werden [68]. Die effektive Länge $l$ des flüssigkeitsgefüllten Koaxialleiterstücks ist dabei wegen des in den Hohlleiter eindringenden evaneszenten Feldes etwas größer als die geometrische Länge des Innenleiters.

Um die Empfindlichkeit des Aufbaus dem Frequenzbereich und der Messsubstanz anzupassen, werden Zellen mit unterschiedlicher Innenleiterlänge verwendet. Für den Frequenzbereich $0.4 \mathrm{MHz} \leq f \leq 100 \mathrm{MHz}$ kam eine Zelle zum Einsatz, deren Innenleiter $10 \mathrm{~mm}$ in die Flüssigkeit hineinreicht. Der Innenleiter der Cut-Off-Zelle, die im Frequenzbereich $20 \mathrm{MHz} \leq f \leq 3 \mathrm{GHz}$ benutzt wurde, hat die geometrische Länge $0 \mathrm{~mm}$, schließt also bündig mit dem Teflonfenster ab.

Bestimmung von Reflexionsfaktor und Dielektrizitätszahl Der in der Steckerebene gemessene Reflexionsfaktor $r(l+L)$ ergibt sich aus dem Zuleitungswiderstand $Z_{0}$ und dem Wellenwiderstand $W(l+L)$ in der Steckerebene:

$$
r(l+L)=\frac{W(l+L)-Z_{0}}{W(l+L)+Z_{0}}
$$

Die Größe $W(l+L)$ lässt sich mit $W(0) \rightarrow \infty$ am kapazitiven Abschluss (s. Abb. 5.3) berechnen. Durch zweimalige Impedanztransformation [69] erhält man [66, 68]:

$$
W(l+L)=Z_{0} \cdot \frac{W(l)+Z_{0} \tanh \left(\gamma_{0} L\right)}{W(l) \tanh \left(\gamma_{0} L\right)+Z_{0}}
$$


mit

$$
W(l)=\frac{Z_{0}}{\sqrt{\epsilon}} \cdot \frac{1}{\tanh \left(\sqrt{\epsilon} \gamma_{0} l\right)}
$$

und der Ausbreitungskonstanten $\gamma_{0}=i \omega / c_{0}$ der ungefüllten Koaxialleitung, der Vakuumlichtgeschwindigkeit $c_{0}$ sowie der Dielektrizitätszahl $\epsilon$ der Flüssigkeit. Der Reflexionsfaktor $r(l+L)$ ist somit durch folgende Gleichung gegeben:

$$
r(l+L)=\frac{1-\tanh \left(\gamma_{0} L\right)}{1+\tanh \left(\gamma_{0} L\right)} \cdot \frac{1-\sqrt{\epsilon} \tanh \left(\sqrt{\epsilon} \gamma_{0} l\right)}{1+\sqrt{\epsilon} \tanh \left(\sqrt{\epsilon} \gamma_{0} l\right)}
$$

Diese Gleichung beschreibt den Zusammenhang von Reflexionsfaktor und Dielektrizitätszahl und bildet die Grundlage des Messverfahrens. Sind $l$ und $L$ bekannt, lässt sich aus den bei verschiedenen Frequenzen gemessenen Reflexionsfaktoren durch numerisches Lösen von Gl. (5.10) die Dielektrizitätszahl $\epsilon(\omega)$ ermitteln.

Um die Unbekannten $l$ und $L$ zu bestimmen, werden nach der Kalibrierung der Netzwerkanalysator/Reflexionstestset-Kombination zwei Referenzmessungen an Substanzen mit bekannter Dielektrizitätszahl vorgenommen. Eine Messung wird mit leerer Messzelle, d.h. mit Luft durchgeführt, die zweite mit einer Substanz, deren dielektrische Eigenschaften im Hinblick auf Dielektriziätszahl und Leitfähigkeit (s.u.) denen der Messflüssigkeit ähneln sollte. Bei den im Rahmen dieser Arbeit durchgeführten Messungen wurde Wasser oder Aceton als Referenz verwendet.

\subsubsection{Fehler des Verfahrens}

In Tabelle 5.1 sind die relativen Fehler der dielektrischen Messungen angegeben. Es handelt sich dabei um Abschätzungen, die auf Mehrfachmessungen und einem Vergleich von Ergebnissen verschiedener Messzellen in deren Überlappbereich basieren. Um systematische Fehler zu reduzieren, wurden die Messdaten in einigen Fällen außerdem gegen unterschiedliche Referenzflüssigkeiten ausgewertet. Im Folgenden werden wesentliche Fehlerquellen diskutiert.

\begin{tabular}{l|c|c} 
Frequenzbereich & Fehler in $\epsilon^{\prime}[\%]$ & Fehler in $\epsilon^{\prime \prime}[\%]$ \\
\hline$\leq 1 \mathrm{GHz}$ & $\leq 1.5$ & $\leq 1.5$ \\
$>1 \mathrm{GHz}$ & $\leq 5$ & $\leq 7$
\end{tabular}

Tabelle 5.1: Relativer Fehler der dielektrischen Messungen

Fehler in der Frequenz Der Fehler in der Frequenz ist bei dem Synthetisiersender des verwendeten NWA vernachlässigbar gering.

Unvollständige Beschreibung der Messzelle Bei der obigen Beschreibung der Messzelle wurden Feldlinien, die sowohl im Teflonfenster als auch in der Flüssigkeit verlaufen, nicht berücksichtigt. Sie führen zu einer zusätzlichen Abhängigkeit des Wellenwiderstandes $W(l)$ von den Dielektrizitätszahlen der Flüssigkeit 
und des Teflonfensters, die sich durch die Einführung von zwei weiteren Kapazitäten ins Ersatzschaltbild beschreiben lässt [68]. Es hat sich gezeigt [68], dass der Einfluss dieser Feldlinien auf den Reflexionsfaktor vernachlässigbar ist, solange die Dielektriziätszahl der untersuchten Flüssigkeit groß genug ist $\left(\epsilon^{\prime}(\omega)>50\right)$. Diese Bedingung ist für nicht zu konzentrierte wässrige Lösungen gut erfüllt. Für Substanzen mit geringerer Polariserbarkeit, wie beispielsweise den untersuchten Polymerschmelzen, ist jedoch durch diesen Effekt mit systematischen Fehlern bei der Bestimmung der Dielektrizitätszahl zu rechnen. Um die entstehenden Fehler abzuschätzen, wurde zum einen auf Übereinstimmung der Messdaten im Überlappbereich beider Messzellen geachtet, zum anderen wurden jeweils zwei Referenzmessungen mit Substanzen unterschiedlicher Dielektrizitätszahl (Wasser und Aceton) durchgeführt. Die Referenz, deren Dielektrizitätszahl näher an der der polymeren Substanz lag, wurde für die Kalibrierung bevorzugt. Dies war in den meisten Fällen Aceton.

Kalibrierung des Netzwerkanalysators Die Kalibrierung des Netzwerkanalysators mit Reflexionstestset kann durch Drift nach einiger Zeit unzureichend werden, was sich vor allem in Fehlern der Messpunkte an den Grenzen des Messbereiches bemerkbar macht. Deshalb ist es nötig, den Netzwerkanalysator bei längeren Betriebszeiten regelmäßig nachzukalibrieren.

Fehler in der Temperatur Die Temperatur der Messproben konnte mit einer Genauigkeit von $0.02^{\circ} \mathrm{C}$ gemessen werden und wurde während der Messung auf Solltemperatur $\pm 0.05^{\circ} \mathrm{C}$ konstant gehalten. Die dadurch entstehenden Fehler in der Dielektriziätszahl sind vernachlässigbar.

Eine signifikante Erwärmung der Flüssigkeit durch das eingekoppelte elektromagnetische Wechselfeld ist wegen der geringen Leistungen nicht zu erwarten. 
5 Dielektrische Spektroskopie 


\section{Die Ergebnisse der dielektrischen Spektroskopie}

Die in diesem Abschnitt beschrieben Ergebnisse wurden bereits in [70] veröffentlicht und im Vergleich zu dielektrischen Spektren anderer Substanzen ausführlich diskutiert. Im Folgenden sollen die für diese Arbeit wesentlichen Aspekte noch einmal herausgestellt werden, wobei vor allem die Frage im Mittelpunkt steht, inwieweit die Fähigkeit der Oligoethylenglykole, Wasserstoffbrückenbindungen einzugehen, die Kettendynamik und die molekulare Struktur der Substanzen beeinflusst.

\subsection{Analytische Beschreibung}

Abbildung 6.1 zeigt das dielektrische Spektrum einer PEG 400/Wasser-Mischung mit einem Wasseranteil von 8 Massenprozent, gemessen bei $10^{\circ} \mathrm{C}$. Dargestellt sind der Realteil $\epsilon^{\prime}(f)$ und der negative Imaginärteil $\epsilon^{\prime \prime}(f)$ der komplexen Dielektrizitätszahl $\epsilon(f)$ als Funktion der Frequenz. Der Verlauf beider Größen ist charakteristisch für die Spektren aller untersuchten Polymerschmelzen und ihrer Mischungen mit Wasser: Im betrachteten Frequenzintervall ist jeweils nur ein ausgeprägter Relaxationsbereich zu erkennen. Dieser Bereich ist zu breit, um von einer DebyeSpektralfunktion mit diskreter Relaxationszeit wiedergegeben werden zu können (s. Abb.6.1), er lässt sich jedoch sehr gut durch eine Cole-Cole-Relaxationsfunktion beschreiben. Die Anpassung der Spektralfunktionen an die Messdaten erfolgte dabei wie in Abschnitt 4.2.1 dargestellt. Zur Berücksichtigung der elektrischen Leitfähigkeit wurde zusätzlich zu den Relaxationsfunktionen ein additiver Term nach Gleichung (5.5) an das Absorptionsspektrum angepasst. Die ermittelte Leitfähigkeit war jedoch in allen Fällen vernachlässigbar klein. Die Ergebnisse der Regressionsanalyse finden sich, zum Teil durch Literaturdaten ergänzt, in den Tabellen $6.1,6.2$ und 6.3 .

\subsection{Diskussion der Ergebnisse}

\subsubsection{Der Verteilungsparameter}

Die Spektren der Oligoethylenglykol-Schmelzen zeigen einen sprunghaften Anstieg des Verteilungsparameters $h$ der Cole-Cole-Spektralfunktion beim Übergang vom Trimer TEG auf das kaum längere PEG 200 (Tab. 6.1). Da der Hersteller für EG, 

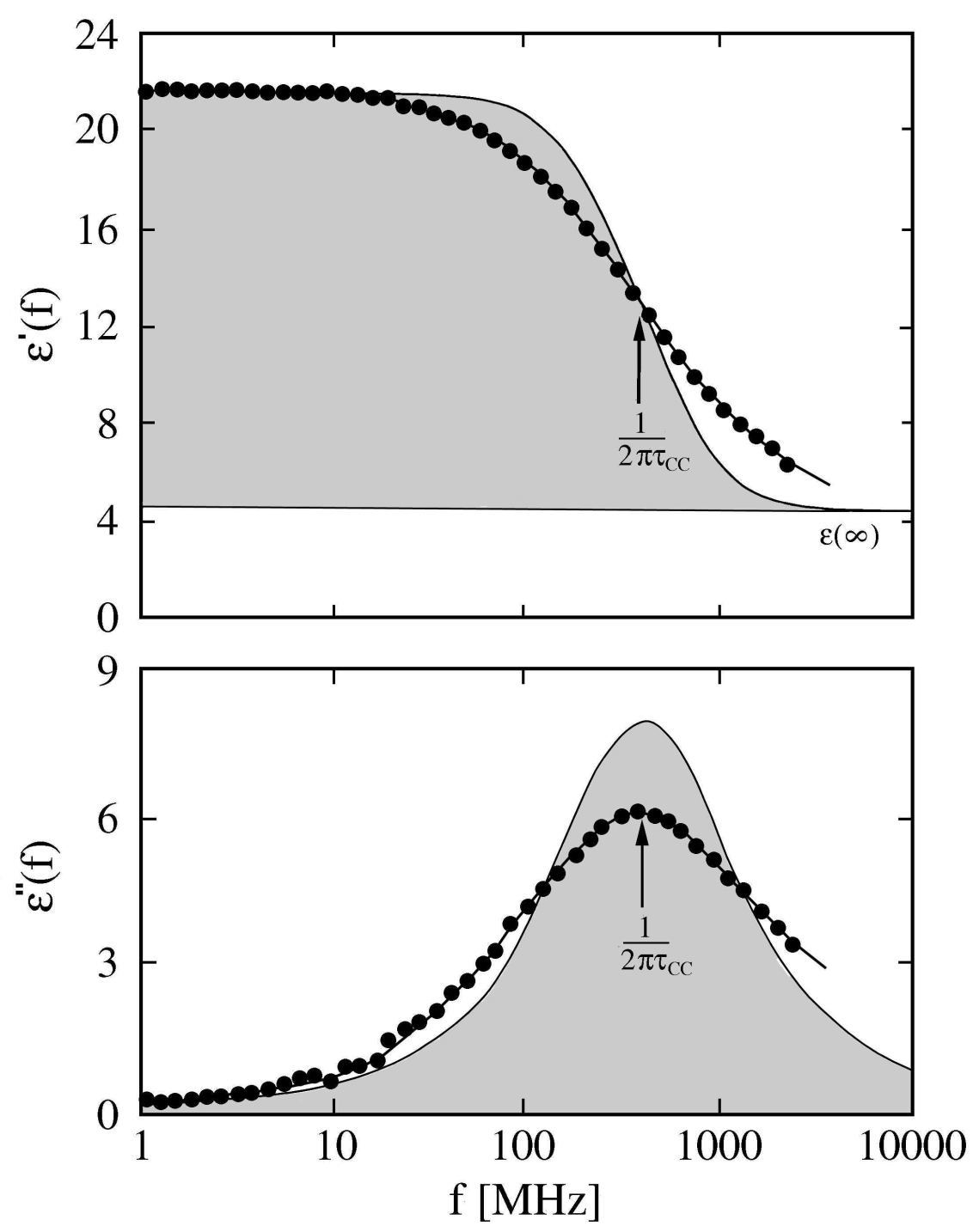

Abbildung 6.1: Realteil $\epsilon^{\prime}(f)$ und negativer Imaginärteil $\epsilon^{\prime \prime}(f)$ der komplexen Dielektrizitätszahl einer PEG 400/Wasser-Mischung mit einem Wasseranteil von 8 Massenprozent als Funktion der Frequenz $f$, gemessen bei $10^{\circ} \mathrm{C}$, beschrieben durch eine Cole-Cole-Relaxationsfunktion mit Hauptrelaxationszeit $\tau_{\mathrm{CC}}$ (durchgezogene Kurve). Graue Flächen illustrieren die Form der Dispersions- und Absorptionsfunktion einer Einzelzeitrelaxation (Verteilungsparameter $h=0$ in Gleichung (5.3)). 


$$
\epsilon(\infty) \quad \epsilon(0) \quad \tau_{\mathrm{CC}}[\mathrm{ps}] \quad h
$$

\begin{tabular}{|c|c|c|c|c|c|}
\hline \multicolumn{6}{|c|}{$25^{\circ} \mathrm{C}$} \\
\hline EG & & $5.8(8)$ & $40.7(2)$ & $121(3)$ & $0.01(2)$ \\
\hline DEG & & $5.8(8)$ & $30.7(2)$ & $237(5)$ & $0.04(2)$ \\
\hline TEG & & $5.5(8)$ & $23.0(2)$ & $205(5)$ & $0.07(2)$ \\
\hline PEG 200 & {$[71]$} & $4.3(8)$ & $19.9(5)$ & $199(6)$ & $0.16(2)$ \\
\hline PEG 300 & {$[71]$} & $3.6(8)$ & $16.2(5)$ & $167(5)$ & $0.22(2)$ \\
\hline PEG 400 (II) & & $3.9(8)$ & $14.3(2)$ & $165(4)$ & $0.18(2)$ \\
\hline PEG 400 & {$[71]$} & $3.3(8)$ & $13.5(5)$ & $140(5)$ & $0.23(2)$ \\
\hline PEG 600 & [71] & $3.2(8)$ & $12.2(5)$ & $120(4)$ & $0.24(2)$ \\
\hline TEGMEE & & $5.1(8)$ & $12.4(2)$ & $87(2)$ & $0.10(2)$ \\
\hline TeEGDME & {$[71]$} & $3.2(5)$ & $8.2(3)$ & $15(1)$ & $0.15(2)$ \\
\hline PEGDME 500 & & $3.1(8)$ & $8.5(2)$ & $31(1)$ & $0.22(2)$ \\
\hline \multicolumn{6}{|c|}{$10^{\circ} \mathrm{C}$} \\
\hline EG & & $5.6(8)$ & $43.9(2)$ & $201(4)$ & $0.02(2)$ \\
\hline DEG & & $5.7(8)$ & $33.1(2)$ & $433(9)$ & $0.05(2)$ \\
\hline TEG & & $5.2(8)$ & $24.4(2)$ & $384(8)$ & $0.08(2)$ \\
\hline PEG 400 (II) & & $3.6(8)$ & $14.9(2)$ & $286(6)$ & $0.27(2)$ \\
\hline TEGMEE & & $5.1(8)$ & $13.4(2)$ & $145(3)$ & $0.11(2)$ \\
\hline TEGDME & & $5.0(8)$ & $8.5(2)$ & $14.6(3)$ & $0.15(2)$ \\
\hline
\end{tabular}

Tabelle 6.1: Parameter aus der Anpassung der dielektrischen Spektren aller untersuchten Polymerschmelzen mit einer Cole-Cole-Spektralfunktion, Werte für $25^{\circ} \mathrm{C}$ und $10^{\circ} \mathrm{C}$. Bezeichnungen der Parameter wie in Abschnitt 5.1.1, der Substanzen wie in Abschnitt 2, TeEGDME: Tetraethylenglykoldimethylether. 


\begin{tabular}{|c|c|c|c|c|c|c|c|c|}
\hline$Y_{\mathrm{W}}$ & $\epsilon(\infty)$ & $\epsilon(0)$ & $\tau_{\mathrm{CC}}[\mathrm{ps}]$ & $h$ & $\epsilon(\infty)$ & $\epsilon(0)$ & $\tau_{\mathrm{CC}}[\mathrm{ps}]$ & $h$ \\
\hline & \multicolumn{4}{|c|}{$25^{\circ} \mathrm{C}$} & \multicolumn{4}{|c|}{$10^{\circ} \mathrm{C}$} \\
\hline 0 & $3.9(8)$ & $14.3(2)$ & $165(4)$ & $0.18(2)$ & $3.6(8)$ & $14.9(2)$ & $286(6)$ & $0.27(2)$ \\
\hline 0.01 & $4.0(8)$ & $14.9(2)$ & $170(4)$ & $0.22(2)$ & $3.5(8)$ & $15.7(2)$ & $325(7)$ & $0.26(2)$ \\
\hline 0.03 & $4.0(8)$ & $16.9(2)$ & $185(4)$ & $0.22(2)$ & $3.9(8)$ & $17.6(2)$ & $342(7)$ & $0.25(2)$ \\
\hline 0.055 & $4.2(8)$ & $18.6(2)$ & $206(5)$ & $0.21(2)$ & $4.2(8)$ & $19.7(2)$ & $373(8)$ & $0.23(2)$ \\
\hline 0.065 & - & - & - & - & $4.3(8)$ & $20.7(2)$ & $388(8)$ & $0.22(2)$ \\
\hline 0.0675 & $4.2(8)$ & $19.6(2)$ & $210(5)$ & $0.19(2)$ & $4.4(8)$ & $20.5(2)$ & $381(8)$ & $0.22(2)$ \\
\hline 0.075 & $4.4(8)$ & $20.5(2)$ & $199(4)$ & $0.19(2)$ & $4.4(8)$ & $21.6(2)$ & $393(8)$ & $0.21(2)$ \\
\hline 0.078 & - & - & - & - & $4.4(8)$ & $22.4(2)$ & $403(9)$ & $0.23(2)$ \\
\hline 0.08 & $4.3(8)$ & $20.6(2)$ & $191(4)$ & $0.19(2)$ & $4.4(8)$ & $21.9(2)$ & $375(8)$ & $0.22(2)$ \\
\hline 0.09 & $4.4(8)$ & $22.3(2)$ & $192(4)$ & $0.19(2)$ & - & - & - & - \\
\hline 0.1 & $4.4(8)$ & $22.9(2)$ & $188(4)$ & $0.19(2)$ & $4.6(8)$ & $23.6(2)$ & $396(2)$ & $0.22(2)$ \\
\hline 0.13 & $4.6(8)$ & $24.8(2)$ & $175(4)$ & $0.18(2)$ & $4.9(8)$ & $25.9(2)$ & $361(8)$ & $0.19(2)$ \\
\hline 0.16 & $4.6(8)$ & $26.2(2)$ & $157(4)$ & $0.16(2)$ & $4.7(8)$ & $29.0(2)$ & $337(7)$ & $0.20(2)$ \\
\hline 0.2 & $4.6(8)$ & $29.9(2)$ & $140(3)$ & $0.17(2)$ & $4.9(8)$ & $31.7(2)$ & $273(6)$ & $0.18(2)$ \\
\hline 0.215 & $4.6(8)$ & $31.1(2)$ & $134(3)$ & $0.17(2)$ & $4.9(8)$ & $32.7(2)$ & $266(6)$ & $0.18(2)$ \\
\hline 0.25 & $4.6(8)$ & $34.5(2)$ & $112(3)$ & $0.16(2)$ & $4.8(8)$ & $35.1(2)$ & $257(6)$ & $0.19(2)$ \\
\hline 0.28 & $4.6(8)$ & $36.0(2)$ & $108(3)$ & $0.15(2)$ & $4.8(8)$ & $37.8(2)$ & $197(4)$ & $0.18(2)$ \\
\hline 0.33 & $4.6(8)$ & $39.4(2)$ & $86.8(2)$ & $0.15(2)$ & $4.8(8)$ & $41.9(2)$ & $160(4)$ & $0.17(2)$ \\
\hline 0.365 & $4.6(8)$ & $41.7(2)$ & $70.4(2)$ & $0.15(2)$ & $4.8(8)$ & $44.4(2)$ & $127(3)$ & $0.16(2)$ \\
\hline 0.44 & $4.6(8)$ & $47.0(2)$ & $54.9(2)$ & $0.12(2)$ & $4.8(8)$ & $49.5(2)$ & $86.5(2)$ & $0.15(2)$ \\
\hline
\end{tabular}

Tabelle 6.2: Parameter aus der Anpassung der dielektrischen Spektren verschiedener PEG 400/Wasser-Mischungen mit einer Cole-Cole-Spektralfunktion. Werte für $25^{\circ} \mathrm{C}$ und $10^{\circ} \mathrm{C}$. Zur Herstellung der Mischungen wurde die Schmelze PEG 400 (II) verwendet. Bezeichnungen wie in 5.1 .1 definiert. $Y_{\mathrm{W}}$ : Massenbruch von Wasser.

\begin{tabular}{lcccc}
$Y_{\mathrm{W}}$ & $\epsilon(\infty)$ & $\epsilon(0)$ & $\tau_{\mathrm{CC}}[\mathrm{ps}]$ & $h$ \\
\hline \hline & & & & \\
\hline 0 & $3.1(8)$ & $8.5(2)$ & $31.3(7)$ & $0.22(2)$ \\
0.05 & $4.8(8)$ & $12.0(2)$ & $69.4(14)$ & $0.14(2)$ \\
0.1 & $4.7(8)$ & $15.5(2)$ & $73.3(15)$ & $0.14(2)$ \\
0.15 & $5.9(8)$ & $19.3(2)$ & $90.0(18)$ & $0.12(2)$ \\
0.2 & $5.3(8)$ & $23.2(2)$ & $82.4(17)$ & $0.11(2)$ \\
0.25 & $5.1(8)$ & $27.3(2)$ & $77.5(16)$ & $0.12(2)$ \\
0.3 & $4.5(8)$ & $31.2(2)$ & $68.3(14)$ & $0.12(2)$ \\
0.4 & $4.0(8)$ & $38.7(2)$ & $48.3(10)$ & $0.12(2)$ \\
0.5 & $3.0(8)$ & $46.3(2)$ & $30.4(7)$ & $0.13(2)$ \\
& & & & \\
\hline
\end{tabular}

Tabelle 6.3: Parameter aus der Anpassung der dielektrischen Spektren verschiedener PEGDME 500/Wasser-Mischungen mit einer Cole-Cole-Spektralfunktion, alle Werte für $25^{\circ} \mathrm{C}$. Bezeichnungen wie in 5.1.1 definiert, $Y_{\mathrm{W}}$ : Massenbruch von Wasser. 


(mittleres)
Substanz $\quad$ Donator-Akzeptor-Verhältnis

\begin{tabular}{lc}
\hline \hline & 1 \\
EG & 0.67 \\
DEG & 0.50 \\
TEG & 0.39 \\
PEG 200 & 0.27 \\
PEG 300 & 0.21 \\
PEG 400 & 0.14 \\
PEG 600 & 0.25 \\
TEGMEE & 0 \\
TEGDME & 0 \\
TeEGDME & 0 \\
PEGDME 500 & \\
\hline
\end{tabular}

Tabelle 6.4: Verhältnis von Wasserstoffbrückendonatoren und Wasserstoffbrückenakzeptoren für die untersuchten Schmelzen. Donatoren sind hier die Wasserstoffatome der OH-Endgruppen, Akzeptoren die Sauerstoffatome mit freien Elektronenpaaren (vergl. auch Tab.2.1 und 2.2).

DEG und TEG noch eine diskrete Kettenlänge, für alle höheren Polymerisationsgrade jedoch nur noch eine Kettenlängenverteilung angeben kann, wird vermutet, dass die erhöhte Verteilungsbreite bei PEG 200 bis 600 auf die Polydispersität dieser Schmelzen zurückzuführen ist. Die Tatsache, dass auch Schmelzen mit wohldefinierter Kettenlänge einen Verteilungsparameter größer als Null besitzen, weist jedoch darauf hin, dass die Polydispersität nicht die einzige Ursache für die den Messdaten zugrunde liegende Relaxationszeitverteilung ist.

Allgemein lässt sich eine Zunahme von $h$ mit wachsender Kettenlänge feststellen. Für die PEG 400/Wasser-Mischungen ist außerdem eine deutliche Abnahme des Verteilungsparameters mit steigendem Wasseranteil zu erkennen. Beide Tendenzen deuten darauf hin, dass die Breite der Relaxationszeitverteilung auch durch das Verhältnis von Wasserstoffbrückendonatoren (Wasserstoffatome der $\mathrm{OH}$ Endgruppen) zu -akzeptoren (Sauerstoffatome mit freien Elektronenpaaren), d. h. durch die Zahl der Möglichkeiten zur Bildung von Wasserstoffbrückenbindungen in der Messsubstanz beeinflusst wird.

Ethylenglykol besitzt mit zwei Sauerstoffatomen und den Wasserstoffatomen der OH-Endgruppen das ausgeglichenste Donator-Akzeptor-Verhältnis der Oligoethylenglykole und weist mit $h=0.01 \pm 0.02$ eine nahezu genauso schmale Relaxationszeitverteilung auf wie Wasser $(h=0.00 \pm 0.01$, [72]). Mit zunehmendem Polymerisationsgrad wächst die Anzahl der Wasserstoffbrückenakzeptoren bei gleichbleibender Anzahl von Donatoren, das Donator-Akzeptor-Verhältnis $c_{\mathrm{d}} / c_{\mathrm{a}}$ verringert sich dementsprechend von 1 bei Ethylenglykol auf 0.14 bei PEG 600 (Tab.6.4), während der Verteilungsparameter von $h=0.01$ auf $h=0.24$ ansteigt (Tab.6.1). Mischt man Wasser zu PEG 400 und erhöht auf diese Weise das Donator-AkzeptorVerhältnis, so nimmt die Breite der Verteilung erwartungsgemäß ab (Tab.6.2). 
Dieses Verhalten findet sich, zumindest für kleine Massenbrüche $Y_{\mathrm{W}}$, auch in den PEGDME 500/Wasser-Mischungen wieder (Tab. 6.3). Für eine Korrelation von Donator-Akzeptor-Verhältnis und Verteilungsparameter spricht außerdem der Anstieg des $h$-Werts vom beidseitig methylierten Derivat TEGDME über TEGMEE mit nur einer OH-Endgruppe hin zu TEG mit zwei OH-Endgruppen (Tab. 6.1, Werte für $10^{\circ} \mathrm{C}$ ). Nicht ganz in dieses Bild scheint PEG 400 (II) zu passen, für das man wegen seines Donator-Akzeptor-Verhältnisses von 0.21 einen geringeren $h$-Wert als für TEGDME mit $c_{\mathrm{d}} / c_{\mathrm{a}}=0$ erwarten würde. Hier könnte zum einen die Polydispersität der PEG 400-Schmelze den hohen Verteilungsparameter erklären, zum anderen ist es denkbar, dass neben dem Donator-Akzeptor-Verhältnis auch noch andere Faktoren die Breite der Relaxationszeitverteilung mit bestimmen.

\subsubsection{Die Hauptrelaxationszeit der Cole-Cole-Spektralfunktion}

Die Hauptrelaxationszeiten $\tau_{\mathrm{CC}}$ der PEG 400/Wasser-Mischungen zeigen einen bemerkenswerten Verlauf (Abb.6.2). Bei $25^{\circ} \mathrm{C}$ liegt die dielektrische (Haupt-)Relaxationszeit von Wasser mit $10 \mathrm{ps}$ deutlich unter dem Wert von reinem PEG 400 $\left(\tau_{\mathrm{CC}}=165 \mathrm{ps}\right)$. Daher könnte man zunächst erwarten, dass die Zugabe von Wasser zu einer Verringerung der Relaxationszeit in der Mischung gegenüber der reinen Schmelze führt. Experimentell ist jedoch für geringe Wasserkonzentrationen zunächst ein Anstieg von $\tau_{\mathrm{CC}} \mathrm{zu}$ beobachten. Erst ab einem Wasseranteil von etwa 8 Massenprozent sinkt die Relaxationszeit mit zunehmendem Wassergehalt monoton ab und nähert sich der Relaxationszeit von reinem Wasser an. Dieses Ergebnis wird durch zeitbereichsspektroskopische Messungen von Sato et al. [73] unterstützt (Abb.6.2). Für die leicht abweichende Lage und Ausprägung des Maximums in den Literaturdaten könnten Unterschiede in der Kettenlängenverteilung der verwendeten Polymerschmelzen verantwortlich sein.

Die Relaxationszeit $\tau_{\mathrm{CC}}$ spiegelt neben der Umorientierung der Wassermoleküle und der OH-Endgruppen des PEG-Moleküls hauptsächlich den Umorientierungprozess der $\mathrm{CH}_{2}-\mathrm{O}-\mathrm{CH}_{2}$-Untereinheiten im Ethylenglykol-Oligomer wider. Ihr unerwarteter Verlauf wird auf zwei gegenläufige Effekte zurückgeführt, die sich beide aus der Fähigkeit von PEG zur Bildung von Wasserstoffbrücken ergeben:

\subsubsection{Erhöhung des Assoziationsgrades durch Zugabe von Wasser}

Der Anstieg der Relaxationszeit bei kleinen Wasserkonzentrationen lässt sich mit der zunehmenden Assoziation der PEG-Moleküle über Wasserstoffbrückenbindungen erklären. Reines PEG 400 verfügt über ein sehr unausgewogenes Verhältnis von Wasserstoffbrückendonatoren und -akzeptoren (Tab. 6.4). Aufgrund der begrenzten Anzahl von Donatoren ist die Wasserstoffbrückenkonzentration gemessen an der Akzeptoranzahl in der Schmelze gering. Mit der Zugabe von Wasser und der damit einhergehenden Erhöhung der Donatorkonzentration entstehen jedoch neue Bindungsmöglichkeiten, so dass sich der Vernetzungsgrad mit zunehmendem 


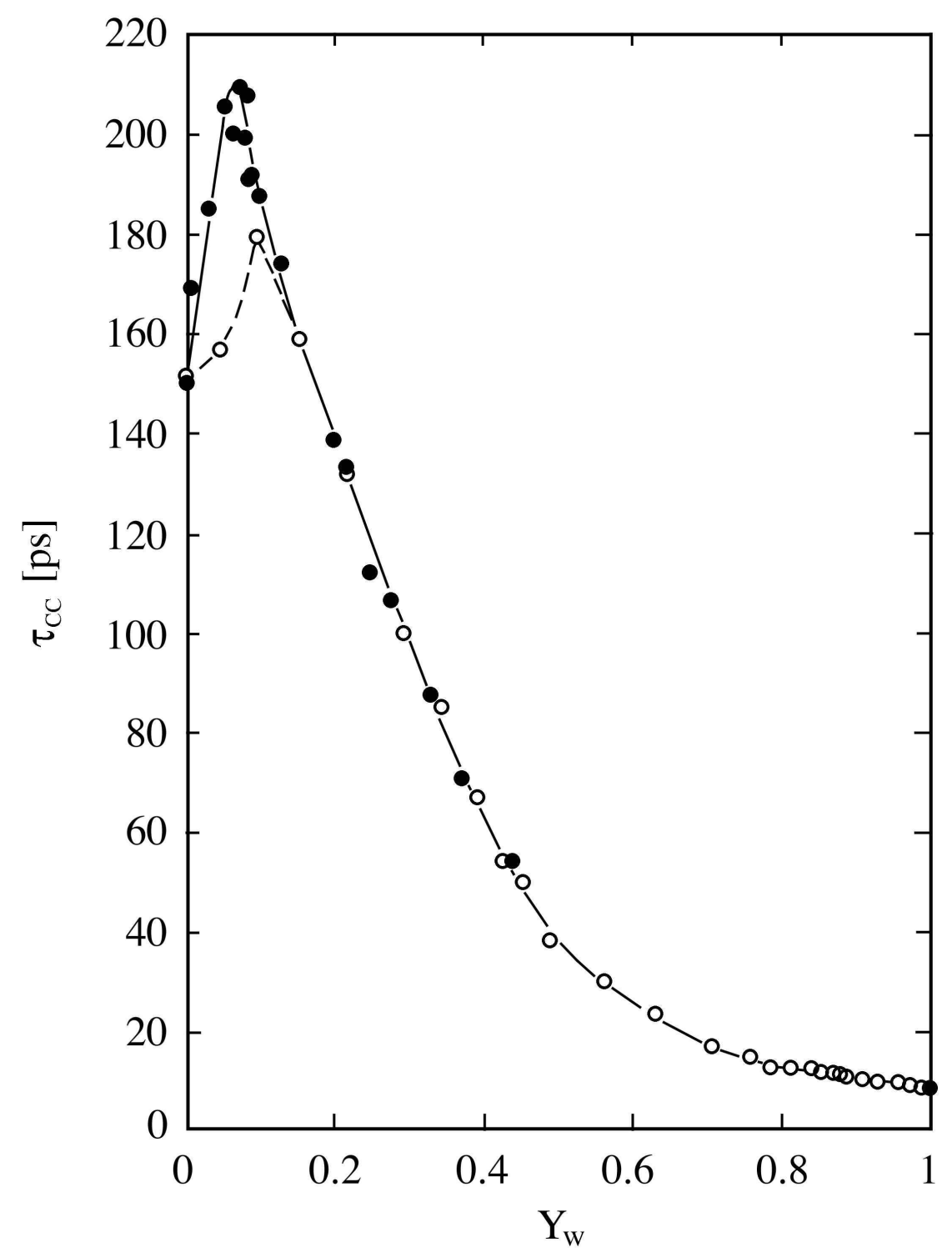

Abbildung 6.2: Hauptrelaxationszeit der PEG 400/Wasser-Mischungen als Funktion des Massenbruchs $Y_{\mathrm{W}}$ von Wasser bei $25^{\circ} \mathrm{C}$. • : eigene Messungen, o: Daten aus [73]. Die durchgezogenen und gestrichelten Kurven sind lediglich Hilfslinien. 
Wassergehalt erhöht. Da ein wasserstoffgebundenes Sauerstoffatom in einer $\mathrm{CH}_{2}-\mathrm{O}-$ $\mathrm{CH}_{2}$-Gruppe sich erst dann umorientieren kann, wenn zuvor die Wasserstoffbrücke aufgebrochen ist, verlangsamt sich der Umorientierungsprozess mit zunehmendem Assoziationsgrad. Dies schlägt sich in einem Anstieg der Relaxationszeit nieder.

Ab einem gewissen Wassergehalt überwiegt jedoch ein anderer Effekt, der trotz der immer ausgeglicheneren Anzahl von Donatoren und Akzeptoren zu einer Abnahme der Relaxationszeit führt. Dieser Effekt wird im folgenden Abschnitt beschrieben.

\subsubsection{Erhöhung der Anzahl alternativer Bindungspartner - "Wait-and-Switch-Modell“"}

Das oben beschriebene Verhalten lässt sich auf der Grundlage eines so genannten „Wait-and-Switch-Modells“ [74-76] besser verstehen. Danach hängt $\tau_{\mathrm{CC}}$ maßgeblich von der Zeit ab, die ein Ensemble von assoziierten Wasserstoffbrückenbindungspartnern innerhalb eines Wasserstoffbrückennetzwerks warten muss, bis sich geeignete Bedingungen für die Umorientierung eines dipolaren molekularen Untereinheit bieten [74]. Computersimulationen [75, 76] zeigen, dass geeignete Bedingungen dann gegeben sind, wenn sich ein zusätzlicher, alternativer Bindungspartner nahe der gebundenen Gruppe in einer günstigen Position befindet. Dieser alternative Bindungspartner stellt dann nicht nur eine neue Bindungsmöglichkeit dar, durch ihn wird auch die Potentialbarriere abgesenkt, die bei der Umorientierung einer dipolaren Gruppe überwunden werden muss. Je mehr alternative Bindungspartner vorhanden sind, desto häufiger ist demnach ein Umorientierungsprozess zu erwarten und desto geringer ist die Relaxationszeit, die diesen Prozess charakterisiert.

Solange jedoch solch eine zusätzliche Bindungsmöglichkeit fehlt, wird sich häufig eine thermisch aufgebrochene Wasserstoffbrückenbindung wieder mit dem alten Bindungspartner erneuern, ohne dass eine signifikante Umorientierung auftritt. In diesem Fall wird $\tau_{\mathrm{CC}}$, wie oben beschrieben, vor allem durch die Wasserstoffbrückenkonzentration bestimmt.

\subsubsection{3 Änderung des Vernetzungsgrades durch Variation der Kettenlänge}

Einen ähnlichen Relaxationszeitverlauf wie bei den PEG 400/Wasser-Mischungen erhält man, wenn man die Zahl der Möglichkeiten zur Bildung von Wasserstoffbrücken anstatt über den Wassergehalt über die Kettenlänge des Oligomers variiert. In Abbildung 6.3 ist die Hauptrelaxationszeit $\tau_{\mathrm{CC}}$ für verschiedene Oligoethylenglykolschmelzen als Funktion des Donator-Akzeptor-Verhältnisses $c_{\mathrm{d}} / c_{\mathrm{a}}$ aufgetragen. Um den Trend für $c_{\mathrm{d}} / c_{\mathrm{a}} \rightarrow 0$ zu berücksichtigen, wurde der Wert von PEGDME 500 mit einbezogen.

Mit steigendem $c_{\mathrm{d}} / c_{\mathrm{a}}$ und somit wachsendem Assoziationsgrad (Tab.6.4) ist auch hier zunächst eine Verlangsamung des Umorientierungsprozesses zu beob- 


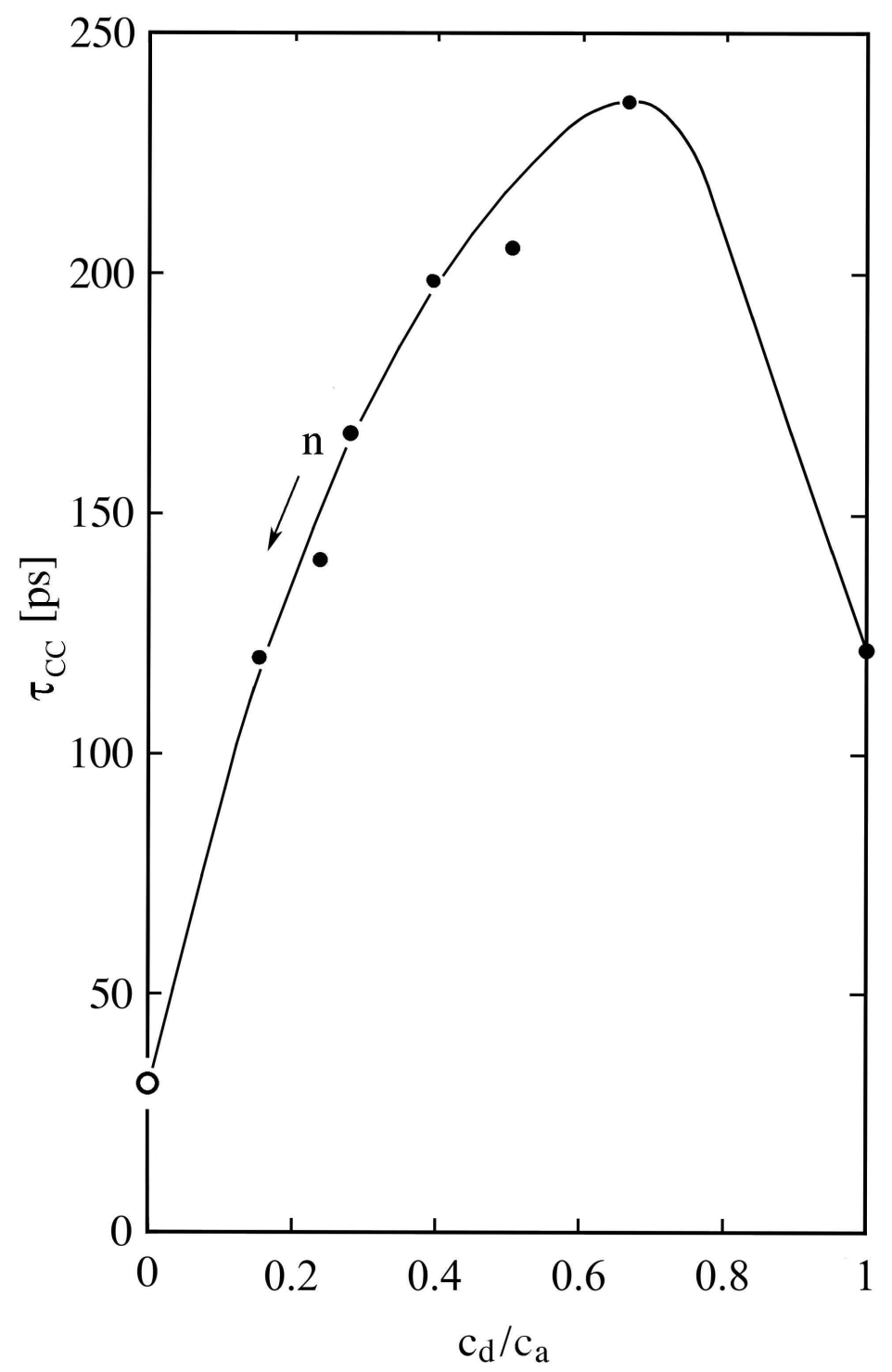

Abbildung 6.3: Hauptrelaxationszeit $\tau_{\mathrm{CC}}$ der untersuchten Oligoethylenglykole als Funktion des Donator-Akzeptor-Verhältnisses bei $25^{\circ} \mathrm{C}$. $\bullet$ : Oligoethylenglykole, o : PEGDME 500, n : Kettenlänge. Verbindungslinie dient nur zur Verdeutlichung des Trends. 
achten. Bei sehr kurzen Ketten ist die Konzentration an alternativen Bindungspartnern dann offenbar so groß, dass sich die Wahrscheinlichkeit für eine Umorientierung, wie bei den Mischungen, wieder erhöht und zu einem Absinken der Relaxationszeit führt.

\subsubsection{Vergleich der Systeme PEG 400/Wasser und PEGDME 500/Wasser}

Auch die Relaxationszeit des Systems PEGDME 500/Wasser zeigt qualitativ die gleiche Konzentrationsabhängigkeit wie die PEG 400/Wasser-Mischungen (Abb. 6.4). Bezogen auf die Relaxationszeit $\tau_{\mathrm{CC}}(0)$ der reinen Schmelze steigt sie jedoch gegenüber den PEG 400/Wasser-Mischungen deutlich stärker an. Ursache hierfür dürfte das unterschiedliche Donator-Akzeptor-Verhältnis der reinen Schmelzen sein. Während PEG bereits im reinen Zustand Wasserstoffbrücken ausbilden kann, ist PEGDME aufgrund fehlender Donatorgruppen in der Schmelze noch unvernetzt. Erst die Zugabe von Wasser ermöglicht den Sauerstoffatomen zu assoziieren. Das Zumischen einer bestimmten Wassermenge wird daher bei PEGDME gegenüber der reinen Schmelze zu einer stärkeren Änderung des Donator-AkzeptorVerhältnisses führen als bei PEG. Entsprechend größer ist auch die beobachtete relative Zunahme der Relaxationszeit im wasserarmen Bereich der PEGDME/WasserMischungen.

\subsubsection{Temperatur- und Kettenlängenabhängigkeit}

In Abbildung 6.5 (großes Bild) ist die Konzentrationsabhängigkeit der Relaxationszeit für die PEG 400/Wasser-Mischungen bei $10^{\circ} \mathrm{C}$ dargestellt. Wieder ist für geringe Wasserkonzentrationen ein Anstieg, für hohe Konzentrationen ein Abfall der Relaxationszeit mit zunehmendem Wasseranteil zu erkennen. Sowohl die absoluten Relaxationszeiten als auch der relative Anstieg von $\tau_{\mathrm{CC}}$ bezogen auf die reine Schmelze sind dabei etwas größer als bei $25^{\circ} \mathrm{C}$. Diese Tendenz steht im Einklang mit dem Temperaturverlauf der Scherviskosität der PEG 400/Wasser-Mischungen (Abb.6.6). Auch hier ist neben einem Anstieg der Viskositäten die immer stärkere Ausprägung eines Maximums mit sinkender Temperatur zu beobachten.

Bemerkenswert am Relaxationszeitverlauf bei $10^{\circ} \mathrm{C}$ ist jedoch das Auftreten eines zusätzlichen lokalen und sehr schmalen Minimums bei einem Wassergehalt von etwa 8 Massenprozent. Die Existenz dieses Minimums konnte durch Wiederholungsmessungen mit unterschiedlichen PEG 400-Chargen bestätigt werden. Es findet sich außerdem in Relaxationszeitmessungen an PEG 600/Wasser-Mischungen wieder ([71] und Abb.6.5, kleines Bild), die mit anderen Apparaturen und Verfahren durchgeführt wurden.

Eine mögliche Erklärung für das Auftreten dieses Minimums ist ein vorkritisches Entmischungsverhalten der PEG/Wasser-Systeme in einem bestimmten Konzentrations- und Temperaturbereich, in dem sich die Mischung aus Mikrophasen mit einer höheren und einer niedrigeren Wasserkonzentration als dem mittleren Wassergehalt zusammensetzt. Für ein solches vorkritisches Verhalten sprechen 


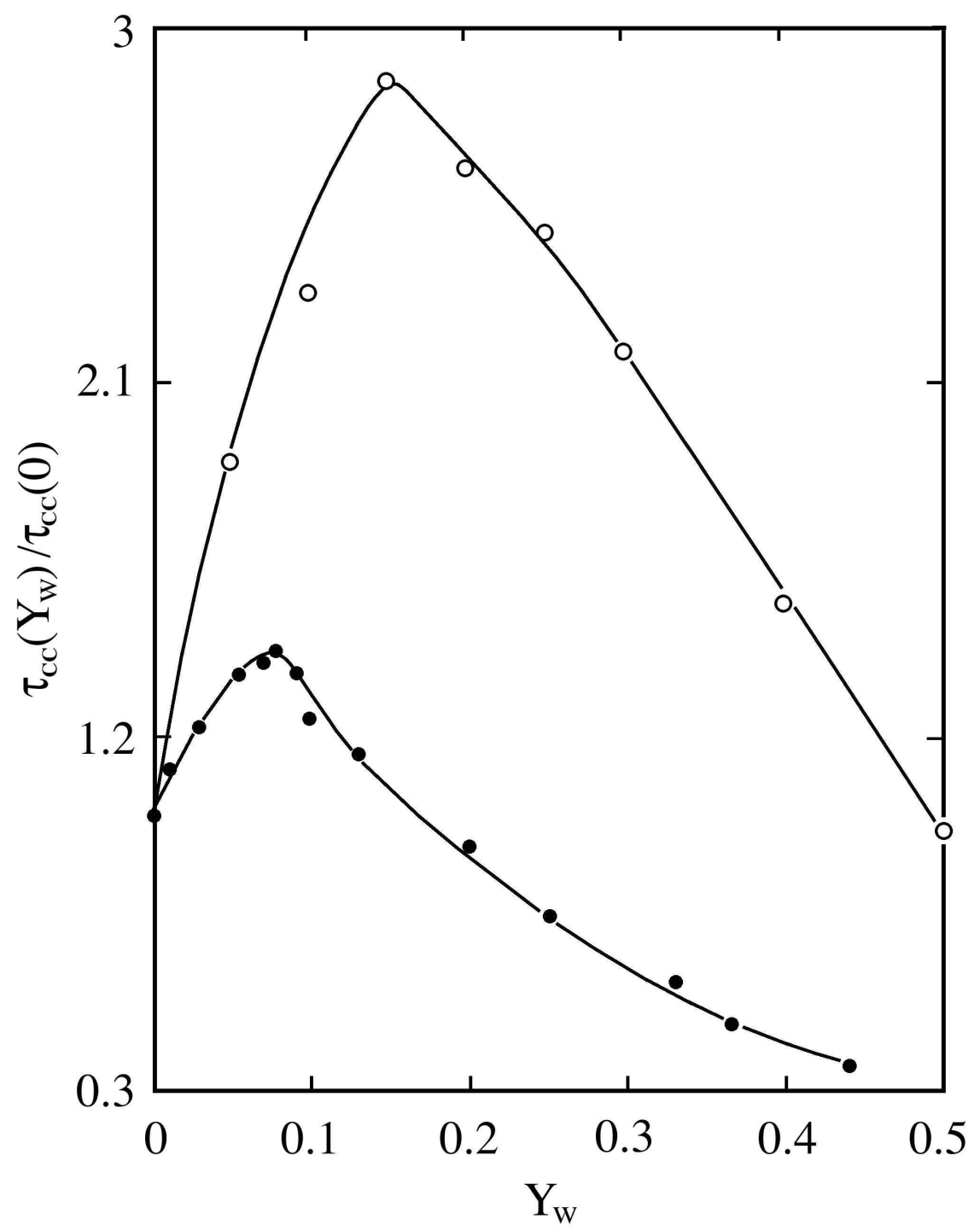

Abbildung 6.4: Gegenüberstellung der relativen Hauptrelaxationszeit $\tau_{\mathrm{CC}} / \tau_{\mathrm{CC}}(0)$ der Systeme PEG 400/Wasser (•) und PEGDME 500/Wasser (o) als Funktion des Massenbruchs $Y_{\mathrm{W}}$ von Wasser bei $25{ }^{\circ} \mathrm{C}$. Durchgezogene Kurven: Hilfslinien. 


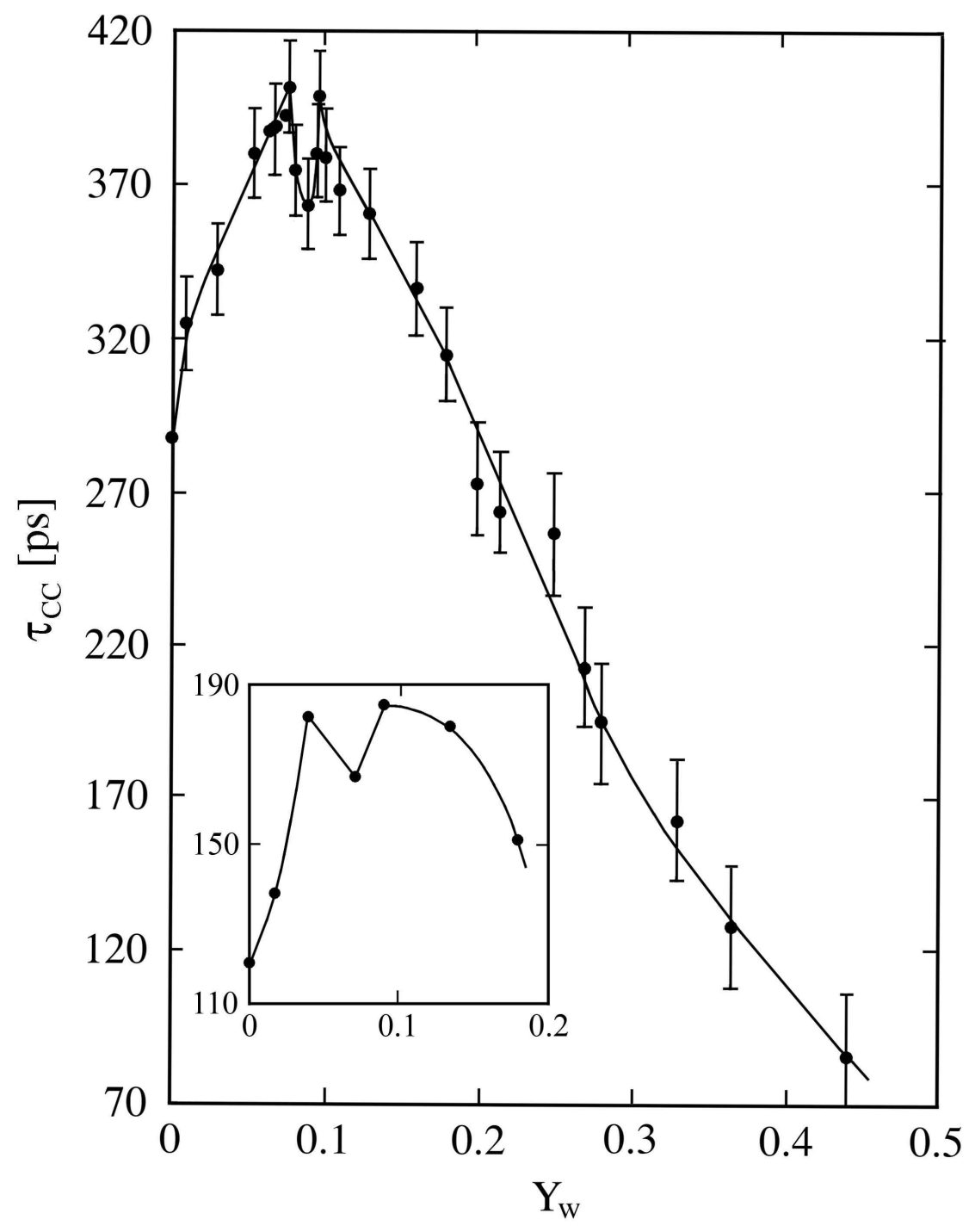

Abbildung 6.5: Großes Bild: Hauptrelaxationszeit $\tau_{\mathrm{CC}}$ der PEG 400/Wasser-Mischungen bei $10^{\circ} \mathrm{C}$ als Funktion des Massenbruchs $Y_{\mathrm{W}}$ von Wasser. Kleines Bild: Hauptrelaxationszeit $\tau_{\mathrm{CC}}$ verschiedener PEG 600/Wasser-Mischungen bei $25^{\circ} \mathrm{C}$ als Funktion von $Y_{\mathrm{W}}$, Daten nach [71]. Durchgezogene Linien : Hilfslinien. 


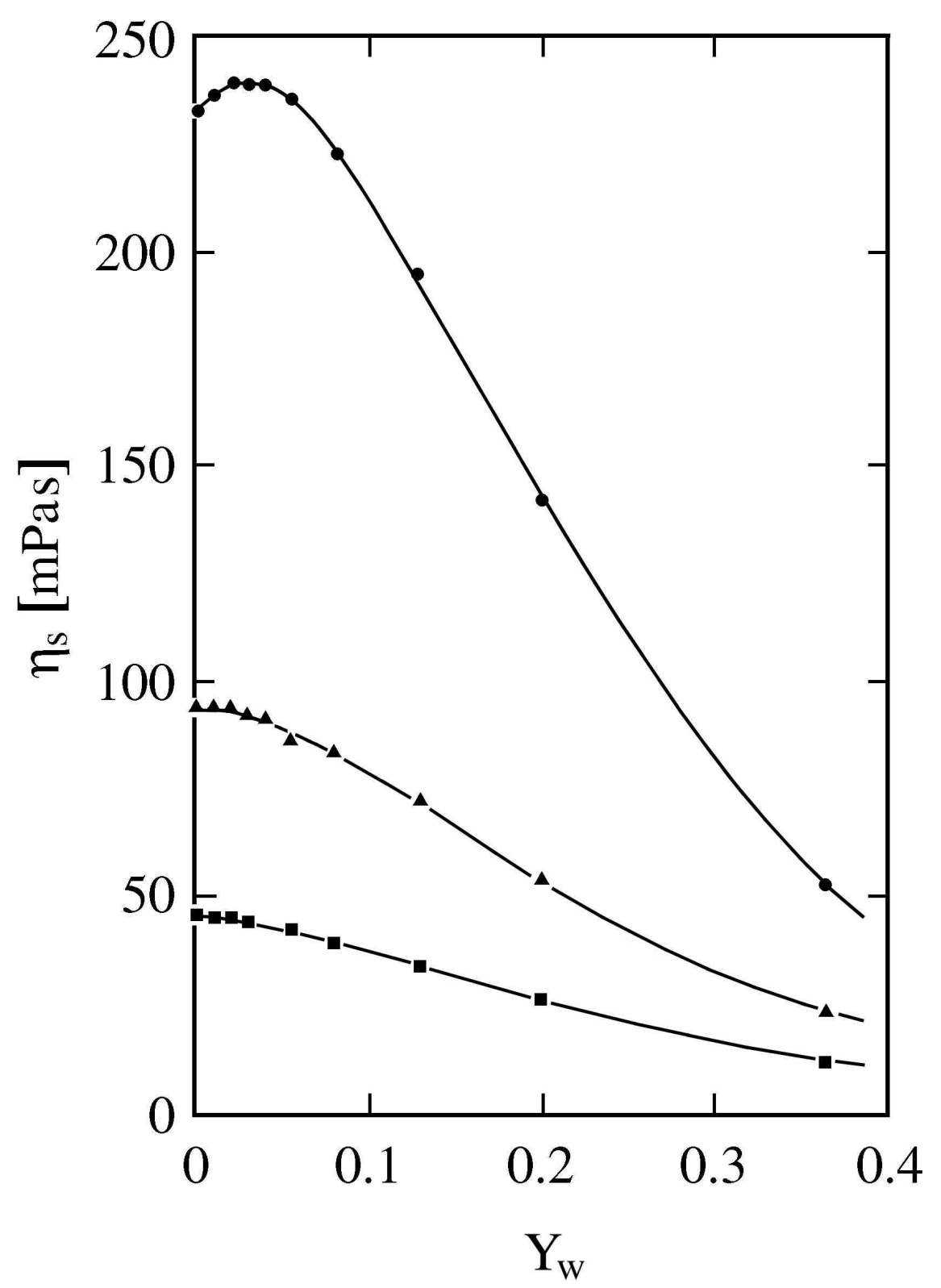

Abbildung 6.6: Scherviskosität $\eta_{\mathrm{s}}$ verschiedener PEG 400/Wasser-Mischungen bei $10^{\circ} \mathrm{C}(\bullet)$, $25^{\circ} \mathrm{C}(\boldsymbol{\Delta})$ und $40^{\circ} \mathrm{C}(\boldsymbol{\square})$. Durchgezogene Linien : Hilfslinien. 
auch Beobachtungen bei Ultraschallabsorptionsmessungen an PEG 400/WasserMischungen (vergl. Abschnitt 7). Dabei traten im Konzentrationsbereich $Y_{\mathrm{W}}=$ 0.04 bis 0.08 wiederholt nach einer gewissen Zeit starke Schwankungen im Absorptionskoeffizienten auf, die zu einem Abbruch der Messungen zwangen. Durch Schütteln der Proben und erneutes Befüllen der Messzelle verschwand der Effekt wieder. Zu keiner Zeit war jedoch eine makroskopische Phasentrennung erkennbar. Neben den experimentellen Beobachtungen unterstützen außerdem neuere Molekulardynamiksimulationen von Mischungen von Wasser und methylierten Polyethylenoxidketten die Idee der Mikrophasenseparation [77]. Nach diesen Berechnungen bilden sich in der Umgebung von $Y_{\mathrm{W}}=0.1$ Wassercluster aus.

\subsubsection{Die zu tiefen Frequenzen hin extrapolierte Dielektrizitätszahl}

Für die reinen Oligoethylenglykol-Schmelzen ist mit wachsender Kettenlänge eine Abnahme der extrapolierten statischen Dielektrizitätszahl $\epsilon(0)$ zu beobachten (Tab.6.1). Dieses Verhalten wird auf die mit wachsender Molmasse kleiner werdende Konzentration dipolarer $\mathrm{OH}$-Endgruppen in der Schmelze zurückgeführt, die ein größeres Dipolmoment und eine größere Dipolmomentdichte besitzen als die monomeren $\mathrm{CH}_{2}-\mathrm{O}-\mathrm{CH}_{2}$-Einheiten der Moleküle (vergl. Abschnitt 6.2.4). Tauscht man die $\mathrm{OH}-E n d g r u p p e n$ vollständig gegen unpolare Methylgruppen aus, zeigt sich entsprechend eine weitere Verringerung der $\epsilon(0)$-Werte. So sinkt beispielsweise $\epsilon(0)$ von 24.4 bei TEG auf 8.5 beim gleich langen TEGDME (Werte für $10^{\circ} \mathrm{C}$, vergl. Tab. 6.1). Eine Erhöhung der Dipolkonzentration durch Zugabe von Wasser äußert sich dagegen in einem Anstieg der extrapolierten statischen Dielektrizitätszahl (Tab. 6.2 und 6.3), in guter Übereinstimmung mit älteren Messergebnissen der Arbeitsgruppen von Sato und Kaatze [71-73, 78], wie Abb.6.7 verdeutlicht.

\subsubsection{Abschätzung der Dipolorientierungskorrelation}

Nach der Fröhlich-Theorie für dipolare Flüssigkeiten [50, 62] ist bei einkomponentigen Systemen die Relaxationsstufe $\epsilon(0)-\epsilon(\infty)$ durch folgenden Ausdruck gegeben:

$$
\epsilon(0)-\epsilon(\infty)=\frac{N_{\mathrm{A}}}{3 \epsilon_{0} k T} \frac{3 \epsilon(0)}{2 \epsilon(0)+\epsilon_{\infty}}\left(\frac{\epsilon_{\infty}+2}{3}\right)^{2} g c \mu^{2} .
$$

Dabei bezeichnet $N_{\mathrm{A}}$ die Avogadro-Konstante, $\epsilon_{0}$ die elektrische Feldkonstante, $k$ die Boltzmann-Konstante und $T$ die absolute Temperatur. Der Parameter $c$ steht für die molare Konzentration permanenter elektrischer Dipole in der Flüssigkeit, $\mu$ für das permanente elektrische Gasdipolmoment der Moleküle. Der von Kirkwood [79] eingeführte Faktor $g$ ist ein Maß für die Orientierungskorrelation zwischen den Dipolen. Ein Korrelationsfaktor $g>1$ weist auf eine vorwiegend parallele Ausrichtung der Dipolmomente, $g<1$ auf eine eher antiparallele Anordnung, $g=1$ auf 


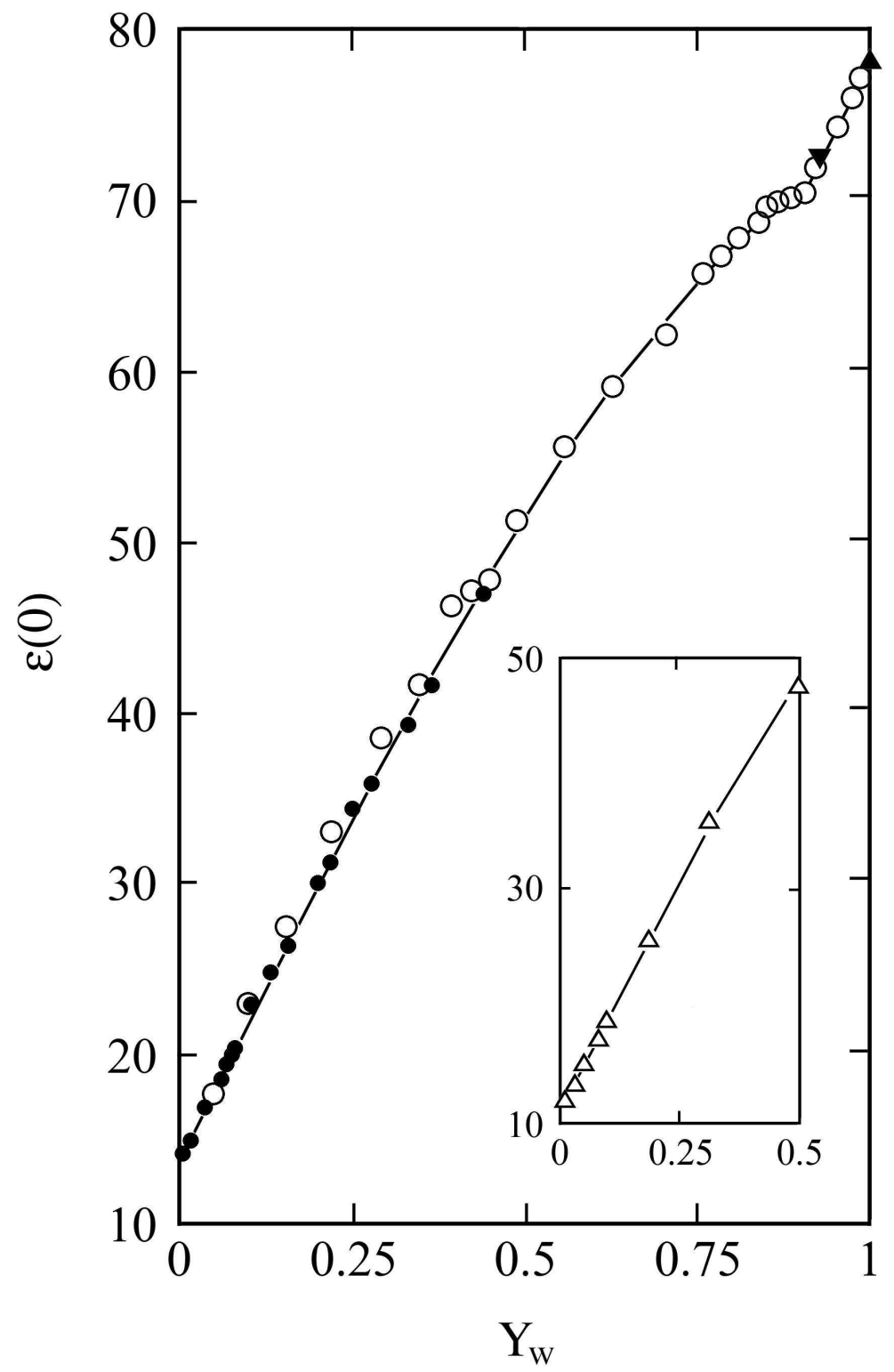

Abbildung 6.7: Zu tiefen Frequenzen hin extrapolierte Dielektrizitätszahl $\epsilon(0)$ für verschiedene PEG 400/Wasser- (großes Bild) und PEG 600/Wasser-Mischungen (kleines Bild) als Funktion des Massenbruchs von Wasser. Alle Werte für $25^{\circ} \mathrm{C}$. $\bullet$ : eigene Messwerte, o: nach [73], $\mathbf{\nabla}:$ nach [78], $\boldsymbol{\Delta}: \operatorname{nach}[72], \Delta: \operatorname{nach}[71]$. 
vollständige Unkorreliertheit der dipolaren Gruppen hin [79]. Durch die hochfrequente Dielektrizitätszahl $\epsilon_{\infty}$ wird die Polarisierbarkeit erfasst, die die Flüssigkeit bei Frequenzen deutlich oberhalb der Orientierungsrelaxation der permanenten Dipolmomente noch besitzt.

Sind $c, \mu$ und $\epsilon_{\infty}$ bekannt, kann aus der gemessenen Relaxationsstufe $\epsilon(0)-\epsilon(\infty)$ der Dipolorientierungskorrelationsfaktor abgeschätzt werden. Während sich Dipolkonzentration und -moment in vielen Fällen gut ermitteln lassen, ist dies bei $\epsilon_{\infty}$ jedoch nicht der Fall. Denn meist kann über den Wert von $\epsilon_{\infty}$ nicht mehr ausgesagt werden, als dass er im Intervall $\left[n^{2}, \epsilon(\infty)\right]$ liegt, wobei $n$ den optischen Brechungsindex bezeichnet. Entsprechend groß ist die Schwankungsbreite in den berechneten Korrelationsfaktoren: Kirkwood setzte für seine Untersuchungen an Wasser $\epsilon_{\infty}=n^{2}=1.33^{2}$ und erhielt mit $g=2.8$ einen Wert, der auf eine vorwiegend parallele Ausrichtung der Dipole schließen lässt. Setzt man dagegen $\epsilon_{\infty}$ mit der extrapolierten hochfrequenten Dielektrizitätszahl $\epsilon(\infty)$ gleich, die sich aus den dielektrischen Messungen von Wasser ergibt, so erhält man mit $g<1$ gerade die entgegengesetzte Tendenz. Hill [62, 80] nahm wiederum an, dass die Dipolmomente in Wasser völlig unkorreliert sind und folgerte daraus einen $\epsilon_{\infty}$-Wert von 4.3. Bei den Spektren der Oligoethylenglykol-Schmelzen wird eine Abschätzung von $g$ zusätzlich durch eine Korrelation zwischen $\epsilon(\infty)$ und dem Verteilungsparameter $h$ erschwert (vergl. Tab.6.1), die auf eine ungenaue Extrapolation der Spektren durch die empirische Cole-Cole-Funktion zu hohen Frequenzen hin zurückgeführt wird [70].

Da der genaue Wert von $\epsilon_{\infty}$ für die untersuchten Spektren nicht bekannt ist, wurde für eine Abschätzung der Dipolorientierungskorrelation zunächst $\epsilon_{\infty}=4.3$ angenommen. Der auf dieser Grundlage bestimmte Korrelationsfaktor wird im Folgenden mit $g_{\text {eff }}$ bezeichnet. Diese Nomenklatur berücksichtigt gleichzeitig, dass mögliche Korrelationen zwischen den unterschiedlichen dipolaren Gruppen in einem Faktor zusammengefasst werden. Die Dipolmomente der Oligomere, die zur Berechnung von $g_{\text {eff }}$ verwendet wurden, sind Tabelle 6.5 zu entnehmen. Die Werte von EG, DEG, TEG und TEGDME beruhen auf Literaturdaten. Für die übrigen Schmelzen wurde aus den Gruppenmomenten $\mu(-\mathrm{COC}-)=1.1 \mathrm{D}[71]$ und $\mu(-\mathrm{COH})=1.7 \mathrm{D}[50]$ ein Näherungswert ermittelt, wobei angenommen wurde, dass sich diese Untereinheiten völlig unabhängig voneinander umorientieren können. In diesem Fall ergibt sich das quadratische Gesamtmoment aus der Quadratsumme der Einzelmomente und liefert in guter Übereinstimmung mit den Literaturdaten für DEG den Wert $\mu=2.64 \mathrm{D}$, für TEG $\mu=2.94 \mathrm{D}$ und für TEGDME $\mu=2.20$ D. Die zur Bestimmung der Dipolkonzentration $c$ notwendigen Dichten sind im Anhang aufgeführt.

Abbildung 6.8 zeigt die berechneten effektiven Korrelationsfaktoren für die untersuchten Oligoethylenglykolschmelzen als Funktion ihres Donator-Akzeptor-Verhältnisses $c_{\mathrm{d}} / c_{\mathrm{a}}$ bei $25^{\circ} \mathrm{C}$. Zusätzlich ist der Wert von PEGDME 500 mit in das Diagramm eingetragen. Alle $g_{\text {eff }}$-Werte bleiben unter 1, was auf eine eher antiparallele Anordnung der dipolaren Gruppen in der Flüssigkeit hindeutet. Mit wachsendem Verhältnis $c_{\mathrm{d}} / c_{\mathrm{a}}$ ist jedoch tendenziell ein Anstieg der Korrelationsfaktoren 
Substanz Dipolmoment [D]

\begin{tabular}{lc}
\hline \hline & \\
EG & $2.28[81, \underline{82}]$ \\
DEG & $2.69[82]$ \\
TEG & $2.99[82]$ \\
PEG 200 & 3.09 \\
PEG 300 & 3.51 \\
PEG 400 & 3.90 \\
PEG 600 & 4.53 \\
TEGMEE & 2.55 \\
TEGDME & $2.16[83]$ \\
TeEGDME & 2.46 \\
PEGDME 500 & 3.70 \\
& \\
\hline
\end{tabular}

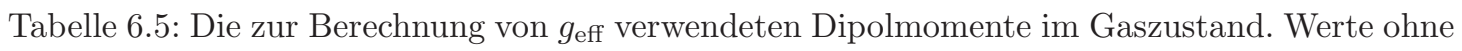
Literaturangaben wurden aus den Dipolmomenten der molekularen Gruppen berechnet.

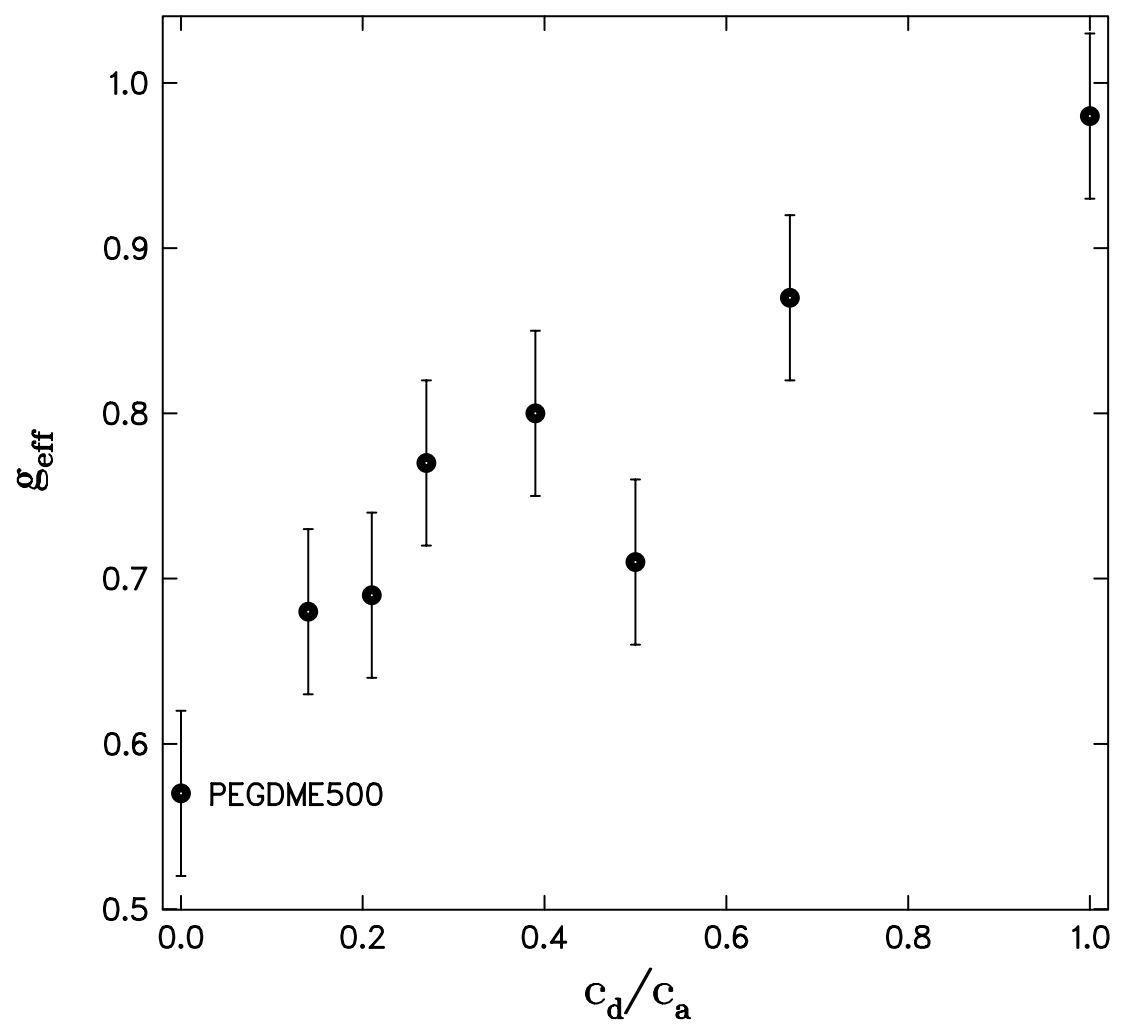

Abbildung 6.8: Effektiver Korrelationsfaktor $g_{\text {eff }}$ der untersuchten Oligoethylenglykolschmelzen und von PEGDME 500 als Funktion des Verhältnisses $c_{\mathrm{d}} / c_{\mathrm{a}}$ von Wasserstoffbrückendonatoren zu -akzeptoren, Werte für $25^{\circ} \mathrm{C}$. Die eingezeichneten Fehlerbalken berücksichtigen Unsicherheiten in $\mu, \rho, \epsilon(0)$ und $\epsilon(\infty)$. Schwankungen aufgrund eines eventuell fehlerhaften $\epsilon_{\infty}$-Wertes sind nicht mit einbezogen. 
zu beobachten. Je ausgeglichener die Anzahl von Donatoren und Akzeptoren im Molekül ist, desto unkorrelierter ist demnach die Ausrichtung der Dipolmomente. Ethylenglykol liegt mit $g_{\text {eff }}=0.98$ dicht an dem Wert $g=1$, der sich unter der Voraussetzung $\epsilon_{\infty}=4.3$ für Wasser ergibt. Dies erscheint sinnvoll, da auch Wasser mit zwei freien Elektronenpaaren und zwei Wasserstoffatomen über ein sehr ausgewogenes Donator-Akzeptor-Verhältnis verfügt. Das Ergebnis dieser Abschätzung sollte aber nicht überbewertet werden, da die Korrelationsfaktoren stark von $\epsilon_{\infty}$ abhängen: Setzt man in Gl. (6.1) für $\epsilon_{\infty}$ das Quadrat des optischen Brechungsindexes ein, bleibt die steigende Tendenz der effektiven Korrelationsfaktoren mit wachsendem Donator-Akzeptor-Verhältnis zwar erhalten, die $g_{\text {eff-Werte, die sich }}$ dann ergeben, sind jedoch alle größer als Eins. Sogar eine völlige Umkehrung des Verlaufs tritt ein, wenn man $\epsilon_{\infty}$ mit dem jeweiligen extrapolierten Wert $\epsilon(\infty)$ gleichsetzt. In diesem Fall würde sich für Ethylenglykol der Faktor $g_{\text {eff }}=0.65$ und für PEGDME $500 g_{\text {eff }}=0.82$ ergeben.

Um die Fröhlich-Relation auf die Polymer/Wasser-Mischungen anzuwenden, wurde Gleichung (6.1) zu folgendem Ausdruck erweitert:

$$
\epsilon(0)-\epsilon(\infty)=\frac{N_{\mathrm{A}}}{3 \epsilon_{0} k T} \frac{3 \epsilon(0)}{2 \epsilon(0)+\epsilon_{\infty}}\left(\frac{\epsilon_{\infty}+2}{3}\right)^{2} g_{\mathrm{eff}} \cdot\left(c_{\mathrm{p}} \mu_{\mathrm{p}}^{2}+c_{\mathrm{w}} \mu_{\mathrm{w}}^{2}\right) .
$$

Dabei bezeichnet $c_{\mathrm{p}}$ die Polymer- und $c_{\mathrm{w}}$ die Wasserkonzentration in der Mischung, $\mu_{\mathrm{p}}$ das Gasdipolmoment des Polymers, $\mu_{\mathrm{w}}=1.85 \mathrm{D}$ [84] das Gasdipolmoment von Wasser. Eigentlich müssten bei der Auswertung der dielektrischen Spektren der Polymer-Wasser-Mischungen drei verschiedene Dipolorientierungskorrelationen berücksichtigt werden, nämlich die zwischen Wassermolekülen, die zwischen Polymermolekülen und eine, die die Polymer-Wasser-Wechselwirkung erfasst. Da aus einer Relaxationsstufe aber nicht drei Korrelationsfaktoren berechnet werden können, wurde der effektive Korrelationsfaktor $g_{\text {eff }}$ in Gl. (6.2) eingeführt. Die $g_{\text {eff }^{-}}$ Werte, die sich mit Hilfe dieser Gleichung und unter der Voraussetzung $\epsilon_{\infty}=4.3$ aus den dielektrischen Spektren der PEG 400/Wasser- und PEGDME 500/WasserMischungen ergeben, sind in Abbildung 6.9 als Funktion des Wassergehalts der Mischung dargestellt.

Auch hier zeigt sich eine Abhängigkeit der Dipolorientierungskorrelation vom Donator-Akzeptor-Verhältnis: Mit steigendem Wassergehalt gleicht sich die Anzahl von Wasserstoffbrückendonatoren und -akzeptoren in der Mischung immer weiter an. Dies führt offenbar, wie bei den PEG-Schmelzen, zu einem Anstieg

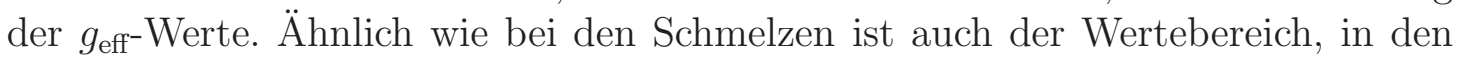
die Korrelationsfaktoren fallen. Sie liegen zwischen 0.5 und 1, wobei die Werte von PEGDME 500 bis zu einer Wasserkonzentration von etwa $Y_{\mathrm{W}}=0.4$ unter denen von PEG 400 bleiben. Ein Grund dafür ist vermutlich, dass PEGDME 500 im Gegensatz zu PEG 400 selbst keine Wasserstoffbrückendonatoren besitzt. Bei vergleichbarem Wasserzusatz wird daher das Donator-Akzeptor-Verhältnis in der PEGDME/Wasser-Mischung stets kleiner sein als in der PEG/Wasser-Mischung und so zu einem geringeren Korrelationsfaktor führen. Dieser Unterschied wird besonders bei kleinen Wasserkonzentrationen eine Rolle spielen und mit steigendem 


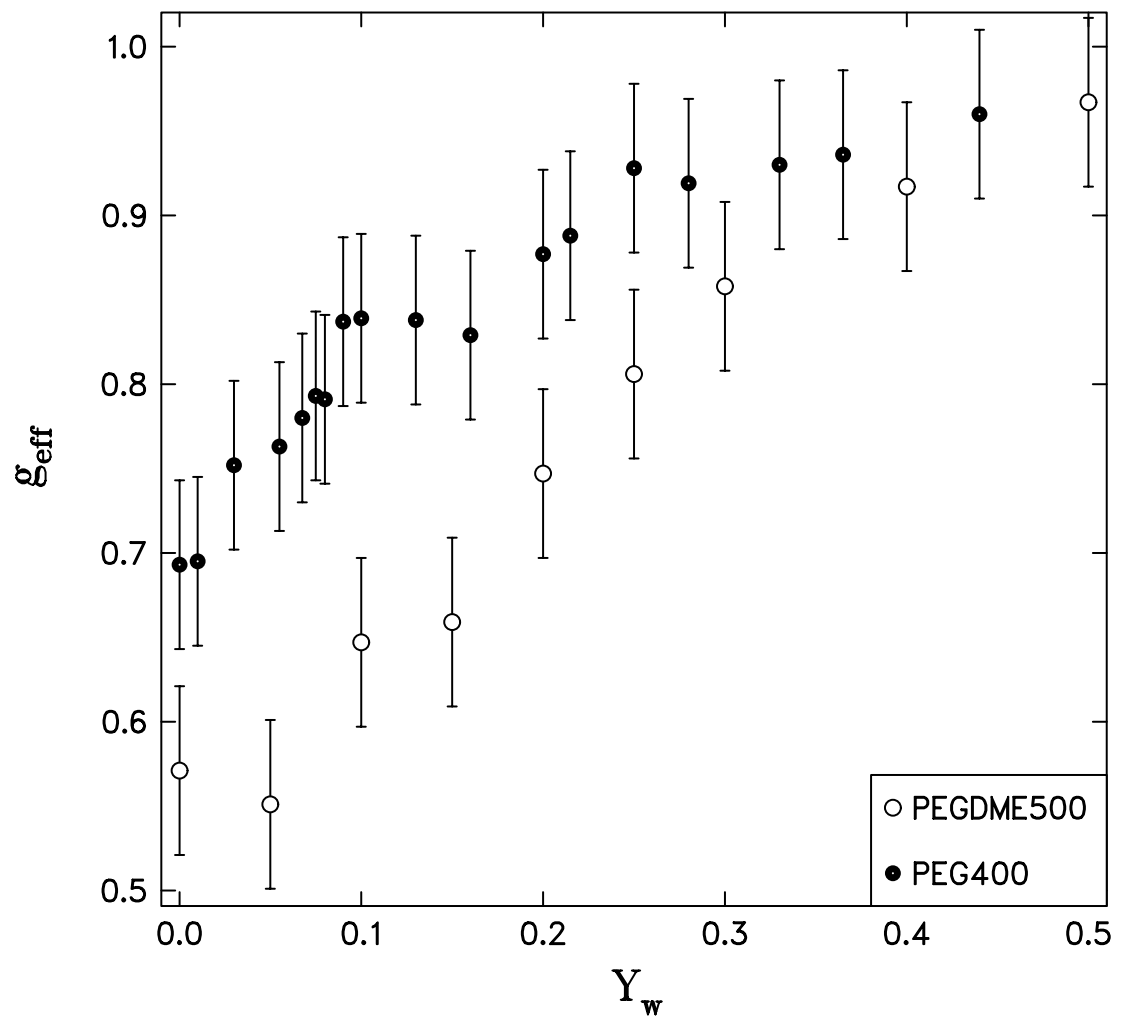

Abbildung 6.9: Effektiver Korrelationsfaktor der Dipolorientierungskorrelation $g_{\mathrm{eff}}$ als Funktion des Massenbruchs $Y_{\mathrm{W}}$ von Wasser für verschiedene Mischungen von Wasser mit PEGDME 500 (॰) und PEG $400(\bullet)$, Werte für $25^{\circ} \mathrm{C}$, Abschätzung der Fehler wie in Abb. 6.8. 
Wassergehalt an Bedeutung verlieren, was die Annäherung der Korrelationsfaktoren im wasserreichen Bereich erklären könnte.

Im Verlauf von $g_{\text {eff }}$ sind sowohl bei beiden Polymer/Wasser-Mischungen als auch bei den Schmelzen mehrere kleine lokale Minima zu erkennen. Man könnte vermuten, dass sich beispielsweise vorkritische Entmischungseffekte, wie sie sich in den Relaxationszeiten der PEG 400/Wasser-Mischungen andeuten, auch in den Richtungskorrelationen der Flüssigkeitsmoleküle zeigen. Aufgrund der großen Fehler in den Abschätzungen von $g_{\text {eff }}$ ist jedoch die tatsächliche Existenz dieser Minima fraglich. Eine weitergehende Diskussion der Korrelationsfaktoren ist daher auf der vorliegenden Datengrundlage nicht sinnvoll. 


\section{Beeinflussung der Kettendynamik durch Wasserstoffbrückenassoziation}

Die in Abschnitt 6 diskutierten dielektrischen Spektren der Ethylenglykol-Oligomere und ihrer Mischungen mit Wasser zeigen deutlich den Einfluss der Wasserstoffbrückenassoziation auf die Umorientierungsdynamik der dipolaren $\mathrm{CH}_{2}$-O$\mathrm{CH}_{2}$-Gruppen in der Flüssigkeit. Es ist daher zu erwarten, dass sich dieser Einfluss auch auf die Bewegungen der gesamten Polymerkette fortsetzt und in den einzelnen Rouse-Moden sichtbar wird. Dies soll im Folgenden durch den Vergleich der Relaxationszeiten aus dielektrischen und akustischen Messungen näher untersucht werden.

\subsection{PEG-Schmelzen}

Besonders naheliegend ist es, den Effekt der Wasserstoffbrückenassoziation zunächst in der jeweils höchsten Rouse-Mode zu suchen, die in den Ultraschallspektren der PEG-Schmelzen beobachtet wurde. In dieser Mode ist die Kette in die maximale Anzahl von Bewegungsabschnitten unterteilt, jedes einzelne Rouse-Segment bewegt sich also gegenphasig zum Nachbarsegment1. Im Fall von PEG setzt sich ein solches Segment gerade aus einer Aneinanderreihung von etwa drei bis vier Kohlenstoff- bzw. Sauerstoffatomen zusammen (Abschnitt 4.3.2.3/Tab. 4.4). Die Bewegung, die ein einzelnes Rouse-Segment in der höchsten Mode ausführt, sollte also ungefähr mit der Umorientierungsbewegung einer $\mathrm{CH}_{2}-\mathrm{O}-\mathrm{CH}_{2}$-Gruppe im dielektrischen Spektrum vergleichbar sein. Aus diesem Grund wurde in Abbildung 7.1 die Cole-Cole-Hauptrelaxationszeit $\tau_{\mathrm{CC}}$ aus den dielektrischen Spektren (Tab. 6.1) mit der (Debye-)Relaxationszeit der höchsten im Ultraschallabsorptionsspektrum beobachteten Rouse-Mode $\tau_{\mathrm{D} 4}$ (Tab. 4.3) für verschiedene PEG-Schmelzen verglichen.

Tatsächlich findet sich die in der dielektrischen Relaxationszeit beobachtete Kettenlängenabhängigkeit tendenziell auch in der höchsten Rouse-Mode wieder. Sowohl $\tau_{\mathrm{CC}}$ als auch $\tau_{\mathrm{D} 4}$ steigen mit wachsendem Polymerisationsgrad zunächst deutlich an, um dann zu größeren Kettenlängen hin langsam wieder abzunehmen. Dabei liegt das Maximum von $\tau_{\mathrm{D} 4}$ bei einer etwas höheren Kettenlänge $(\bar{n}=4.1)$. Etwas

\footnotetext{
${ }^{1}$ entsprechend einer Feder-Masse-Kette, deren Federn (räumlich) abwechselnd kontrahiert und expandiert vorliegen (vergl. Abschnitt 4.3.1).
} 


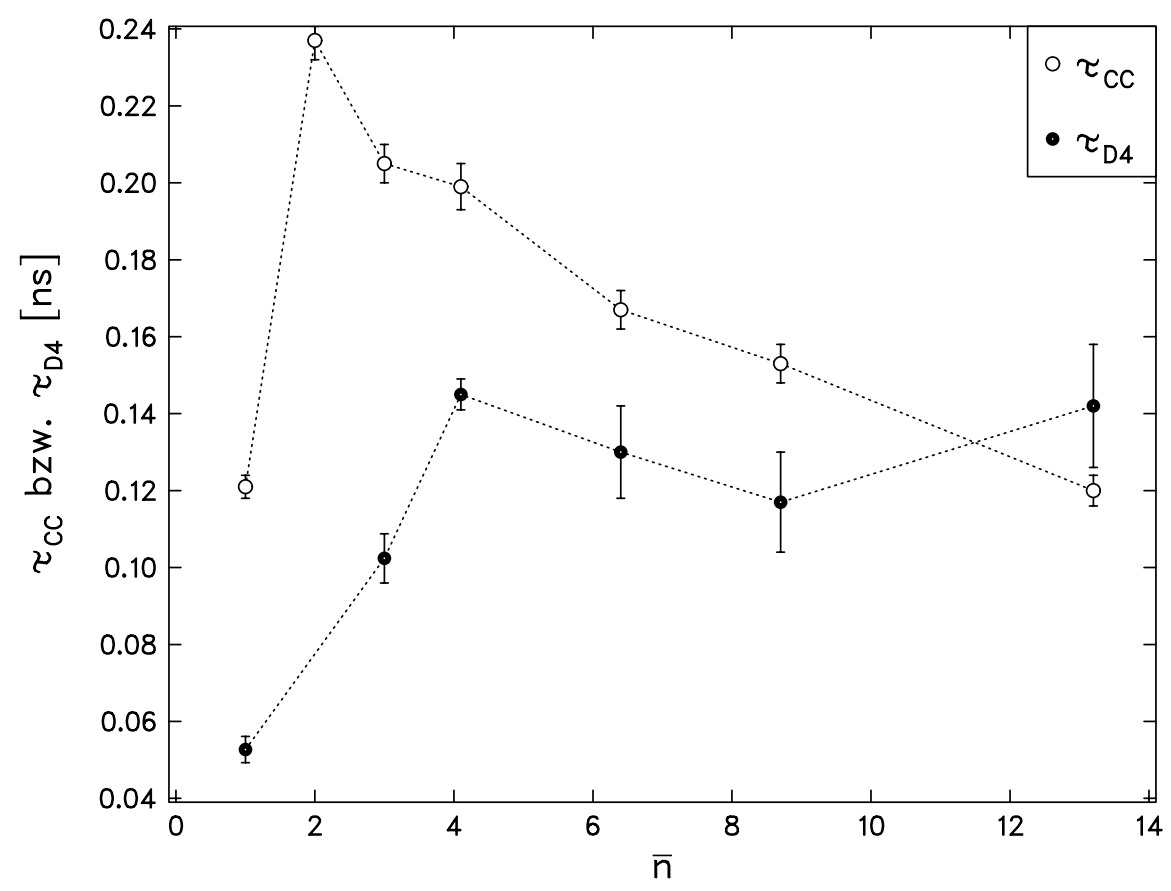

Abbildung 7.1: Hauptrelaxationszeit $\tau_{\mathrm{CC}}$ aus der Cole-Cole-Beschreibung der dielektrischen Spektren (Tab. 6.1) und die (Debye-)Relaxationszeit der höchsten im Ultraschallabsorptionsspektrum beobachteten Rouse-Mode $\tau_{\mathrm{D} 4}(\mathrm{Tab} .4 .3)$ bei $25^{\circ} \mathrm{C}$ als Funktion der Kettenlänge für verschiedene PEG-Schmelzen. $\bar{n}$ : mittlere Anzahl monomerer Einheiten. Gestrichelt: Hilfslinien.

aus der Reihe zu fallen scheint die Relaxationszeit $\tau_{\text {D4 }}$ von PEG $600(\bar{n}=13.2)$. Der (zu) hohe Wert wird jedoch auf die unzureichende analytische Beschreibung des zugehörigen Ultraschallabsorptionsspektrums zurückgeführt: Nach Tabelle (4.4) wären für PEG 600 eigentlich sechs (ungerade) Moden zu erwarten. Für eine verlässliche Beschreibung des Spektrums mit sechs Debye-Spektralfunktionen ist der untersuchte Frequenzbereich jedoch zu schmal (vergl. Abschnitt 4.3.2.1). Um noch stabile und sinnvolle Regressionsergebnisse gewährleisten zu können, wurden maximal vier Debye-Funktionen an die Messdaten angepasst. Dies hat jedoch zur Folge, dass besonders die eng nebeneinander liegenden, schlecht aufösbaren hohen Moden im oberen Rand des Messbereichs in Mischtermen zusammengefasst werden. Vor allem in den Parametern der hochfrequenten Einzelzeitrelaxationen ist daher im PEG600-Spektrum mit großen systematischen Fehlern zu rechnen, wie sich auch schon in den Untersuchungen des Zusammenhangs von Kettenlänge und Relaxationszeit bei fester Modenordnung (Abschnitt 4.3.2.1) gezeigt hat.

Für die zweithöchste Rouse-Mode steht zwar noch ein Messwert weniger zur Verfügung als bei der höchsten Mode, und der Wert von PEG 600 weicht, vermutlich aus den denselben Gründen wie bei $\tau_{\mathrm{D} 4}$, vom erwarteten Trend ab, dennoch deutet sich auch hier in guter Übereinstimmung mit den Ergebnissen aus den dielektrischen Messungen ein Maximum im kettenlängenabhängigen Verlauf der Relaxationszeit $\tau_{\mathrm{D} 3}$ an (Abb. 7.2).

Wie vermutet, beeinflusst die Assoziation über Wasserstoffbrücken also offenbar 


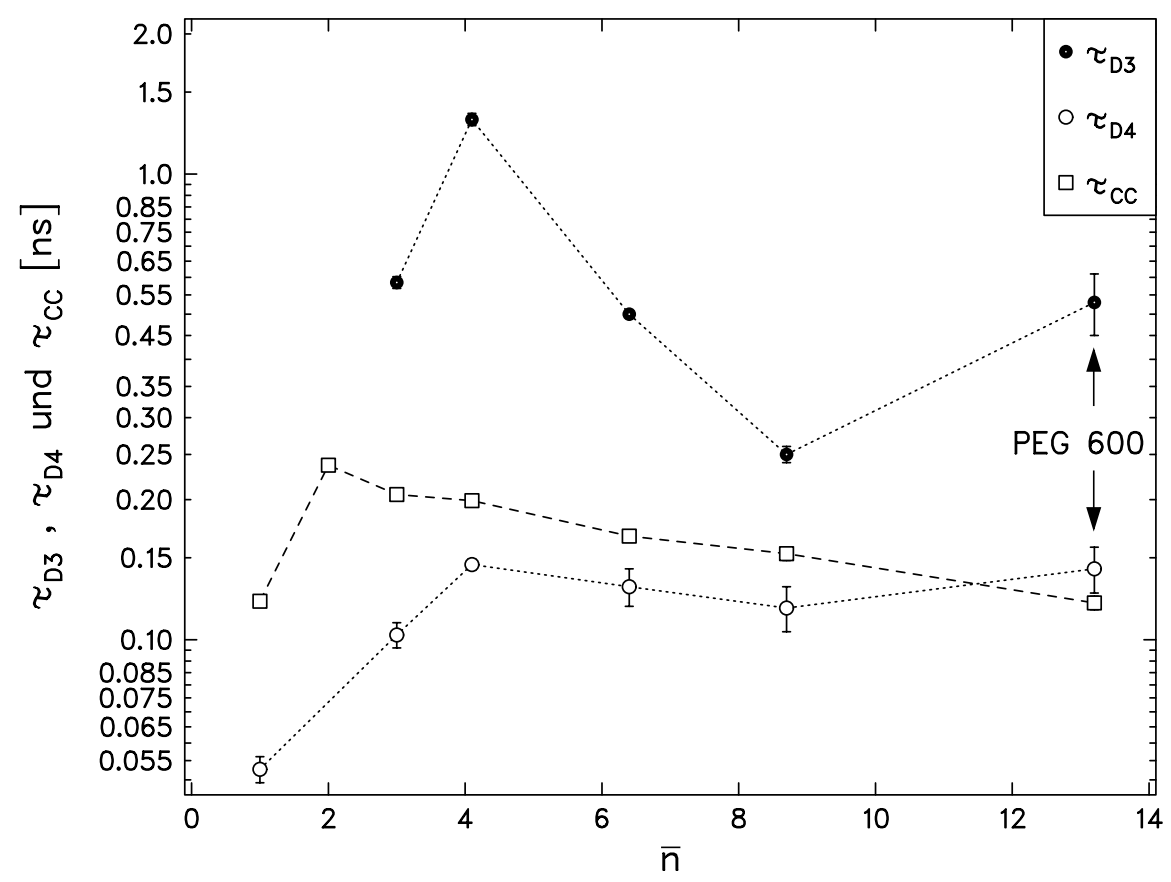

Abbildung 7.2: Relaxationszeit der höchsten und zweithöchsten Rouse-Mode im Vergleich mit der dielektrischen Relaxationszeit $\tau_{\mathrm{CC}}$ für die untersuchten PEG-Schmelzen. $\tau_{\mathrm{D} 4}$ : Relaxationszeit der höchsten beobachteten Mode, $\tau_{\mathrm{D} 3}$ : Relaxationszeit der zweithöchsten beobachteten RouseMode, Werte für $25^{\circ} \mathrm{C}$ nach Tab.4.3 und Tab.6.1. Gestrichelte und gepunktete Linien sind als Hilfslinien zu verstehen.

auch die Rouse-Moden: Wenn von größeren Kettenlängen kommend das Verhältnis von Wasserstoffbrückendonatoren zu -akzeptoren zunimmt, erhöht sich mit dem Vernetzungsgrad auch die Relaxationszeit $\tau_{\mathrm{D} 4}$, da die Bewegung eines assoziierten Kettensegments durch die Wasserstoffbrückenbindung etwas behindert wird. Bei kleinen Kettenlängen hat sich die Konzentration an alternativen Bindungspartnern dann so weit erhöht, dass die Wahrscheinlichkeit für eine Dreh- oder Translationsbewegung eines Kettensegments wieder ansteigt und die Relaxationszeit wieder abnimmt (vergl. Abschnitt 6.2.2).

\subsection{PEG/Wasser-Mischungen}

Um einen Einblick zu gewinnen, inwieweit sich die Polymerdynamik durch Zugabe von Wasser zu den PEG-Schmelzen ändert, wurden Ultraschallabsorptionsmessungen an einigen PEG 400/Wasser-Mischungen über einen Konzentrationsbereich von 4 bis 60 Massenprozent Wasser vorgenommen. Bei deren Interpretation besteht das folgende Problem: Voraussetzung für die Gültigkeit des Rouse-Modells ist eine verschwindende Volumenausschlusswechselwirkung (Abschnitt 4.3.1). Diese Voraussetzung ist jedoch nur in Polymerschmelzen und $\theta$-Lösungen erfüllt. Zwar existieren auch in den unterschiedlichen PEG/Wasser-Mischungen noch Bewegungsmoden, welchen Gesetzmäßigkeiten sie in den unterschiedlichen Konzentrationsbe- 
reichen folgen, ist allerdings unklar. Um eine Überinterpretation der Spektren zu vermeiden, wurden die Messdaten immer durch eine möglichst kleine Anzahl von Debye-Spektralfunktionen beschrieben, auch wenn die Ergebnisse aus Abschnitt 4 auf die Existenz weiterer Einzelzeitrelaxationsprozesse hinweisen. So wurden an die Spektren maximal drei Debye-Spektralfunktionen angepasst, obwohl für reines PEG 400 vier Rouse-Moden zu erwarten wären (s. Tab. 4.4). Insbesondere im hochfrequenten Bereich ist dementsprechend damit zu rechnen, dass möglicherweise nicht alle vorhandenen Relaxationsprozesse aufgelöst werden. Unter der Voraussetzung, dass sich alle Moden bei Zugabe von Wasser in ähnlicher Weise verändern, kann man aber dennoch erwarten, dass sich das konzentrationsabhängige Verhalten der einzelnen Moden tendenziell auch noch in den Überlagerungen dieser Prozesse wiederfindet.

Da für die Mischungen das vermutlich verunreinigte PEG 400 (I) verwendet wurde, musste ein zusätzlicher Relaxationsprozess nach Abschnitt 4.2.3.1 berücksichtigt werden. Dieser scheint jedoch nach Zugabe von Wasser zu verschwinden, denn bereits bei einem Wasseranteil von vier Massenprozent konnte er nicht mehr detektiert werden.

Besonders schwierig ist die Auswertung der Daten für die Mischungen mit 30 und 60 Massenprozent Wasser. Diese Messungen wurden zu einem deutlich späteren Zeitpunkt durchgeführt als alle anderen. Noch ungeklärte Probleme mit der Hyperschall-Messapparatur verursachen hier einen Versatz der hochfrequenten Daten gegenüber den Messwerten der hochfrequenten Pulsmesszelle. Auch bei Referenzmessungen an Wasser liegt der bestimmte Absorptionskoeffizient immer etwa sieben Prozent über dem Erfahrungswert. Es wurde versucht, die dadurch entstehenden Unsicherheiten im hochfrequenten Spektralbereich abzuschätzen. Sie wurden mit in die Fehler der Parameter einbezogen.

Eine weiteres Problem, das bereits bei der Diskussion der dielektrischen Spektren erwähnt wurde, trat bei den Messungen an den Mischungen mit einem Wasseranteil von vier und acht Massenprozent auf. In diesem Konzentrationsbereich sind reproduzierbar einige Stunden nach dem Befüllen der Pulstransmissionsmesszellen starke Schwankungen im Absorptionskoeffizienten zu beobachten, die typisch für Entmischungsvorgänge sind. Die Flüssigkeit ist jedoch weiterhin klar und zeigt keine Anzeichen einer makroskopischen Phasentrennung. Vermutet wird, dass sich in diesem Konzentrationsbereich Mikrophasen mit höheren bzw. niedrigeren Wasseranteilen als der mittleren Wasserkonzentration bilden. Für diese Vermutung sprechen auch Simulationsergebnisse von Borodin et al. [77], die für methyliertes Polyethylenoxid die Bildung von Wasserclustern im Bereich von $Y_{\mathrm{W}}=0.1$ vorhersagen. Um eine Messreihe überhaupt vollständig durchführen zu können, musste die Flüssigkeit zum Teil mehrfach abgezogen, geschüttelt und wieder neu eingefüllt werden. Zwar wurden nach einer Neubefüllung und vor dem Auftreten der Entmischungseffekte jeweils wieder die gleichen Absorptionskoeffizienten gemessen, da diese Mischungen aber offenbar nicht in einem stabilen Zustand vorliegen, sind die Ergebnisse mit einer gewissen Vorsicht zu betrachten.

In Tabelle 7.1 sind die Parameter, die sich aus der Regressionsanalyse der PEG 


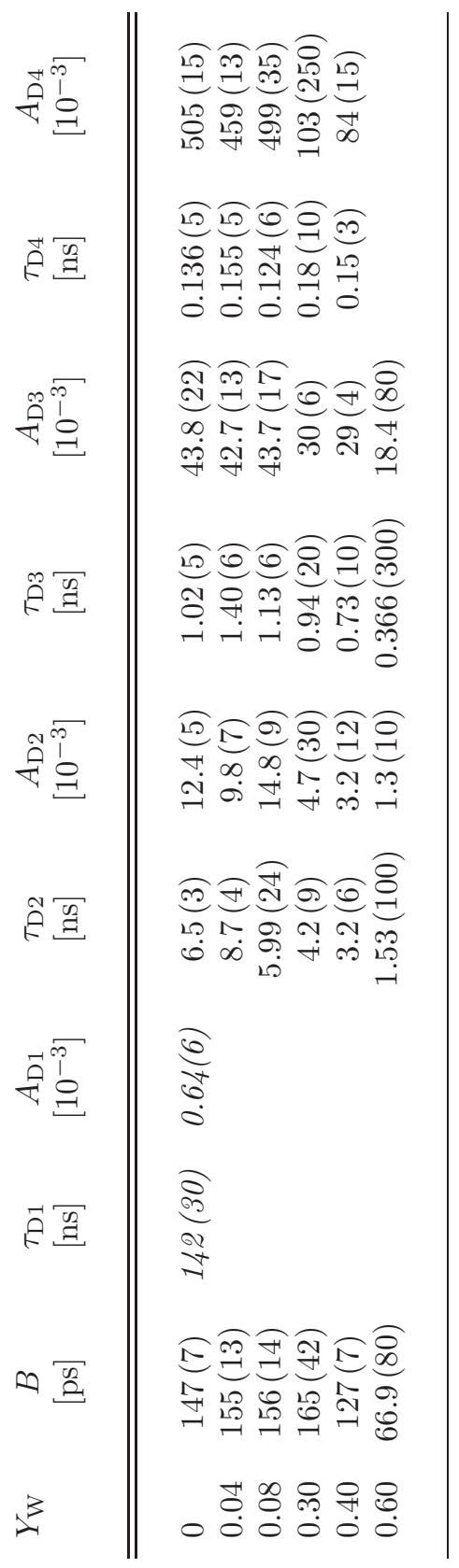

Tabelle 7.1: Parameter aus der Regressionsanalyse der Ultraschallabsorptionsspektren von PEG 400 (I)/Wasser-Mischungen mit Debye-Spektralfunktionen. Alle Werte für $25^{\circ} \mathrm{C}$. Verwendet wurden die nicht dispersionskorrigierten Messwerte. Bei den Fehlern wurden die dispersionskorrigierten und, soweit vorhanden, die gegen Polymerreferenz ausgewerteten Daten mit einbezogen, außerdem werden bei $Y_{\mathrm{W}}=0.3$ und $Y_{\mathrm{W}}=0.6$ die durch den nicht vollständigen Anschluss der Hyperschallmesspunkte verursachten hohen Unsicherheiten im hochfrequenten Messbereich berücksichtigt. $Y_{\mathrm{W}}$ : Massenbruch von Wasser, $B: B$-Wert nach Gl. (3.10), $\tau_{\text {Di }}$ : Relaxationszeit der $i$-ten Einzelzeitrelaxation, $A_{\mathrm{Di}}$ : Relaxationsamplitude der $i$-ten Einzelzeitrelaxation, Kursivdruck: in Abschnitt 4.2.3.1 diskutierter Zusatzterm. Nummerierung der Prozesse von großen zu kleinen Relaxationszeiten, Prozesse mit ähnlichen Relaxationszeiten und Amplituden sind gemeinsamen Spalten zugeordnet. 


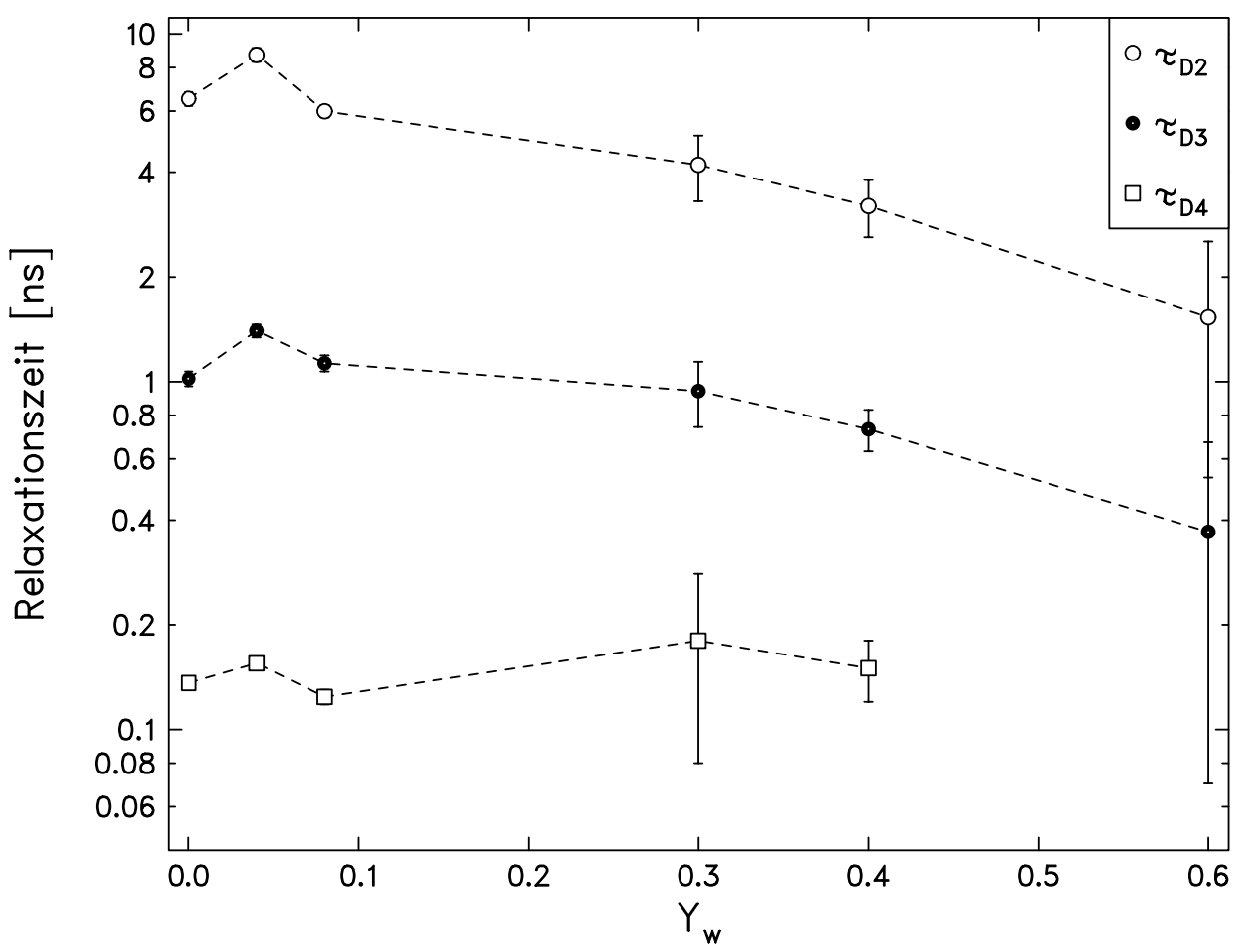

Abbildung 7.3: Relaxationszeiten $\tau_{\mathrm{Di}}, i=2,3,4$, nach Tab. 7.1 für die verschiedenen PEG 400/Wasser-Mischungen, dargestellt als Funktion des Massenbruchs $Y_{\mathrm{W}}$ von Wasser. Alle Werte für $25^{\circ} \mathrm{C}$. Gestrichelt: Hilfslinien.

400/Wasser-Spektren ergeben haben, aufgelistet. Wegen der oben diskutierten großen Unsicherheiten in einigen Spektren und mangels eines für alle Mischungsverhältnisse gleichermaßen geeigneten theoretischen Modells wird im Folgenden lediglich das konzentrationsabhängige Verhalten der Relaxationszeiten für die beobachteten Prozesse diskutiert.

\subsubsection{Konzentrationsabhängiger Verlauf der Relaxationzeiten}

Ausgehend von den Ergebnissen der akustischen Spektroskopie an PEG-Schmelzen (Abschnitt 4) werden die beobachteten Einzelzeitrelaxationen D2, D3 und D4 als Bewegungsmoden der Polymerketten aufgefasst, die mit den Rouse-Moden vergleichbar sind. Ihre Relaxationszeiten $\tau_{\mathrm{D} 2}, \tau_{\mathrm{D} 3}$ und $\tau_{\mathrm{D} 4}$ zeigen in Abhängigkeit vom Wassergehalt einen Verlauf, der erneut auf eine Beeinflussung der Polymerkettenbewegung durch die Wasserstoffbrückenassoziation hinweist. Während man wegen der geringeren Viskosität von Wasser eher eine Abnahme der Relaxationszeit mit steigendem Wassergehalt erwarten könnte, ist bei Zugabe geringer Wassermengen zunächst ein leichter Anstieg in $\tau_{\mathrm{D} 2}, \tau_{\mathrm{D} 3}$ und $\tau_{\mathrm{D} 4} \mathrm{zu}$ erkennen (Abb. 7.3). Erst oberhalb eines Wasser-Massenbruchs von 0.04 nehmen die Relaxationszeiten tendenziell mit wachsendem $Y_{\mathrm{W}}$ ab. Die Maxima sind zwar nur schwach ausgeprägt, 


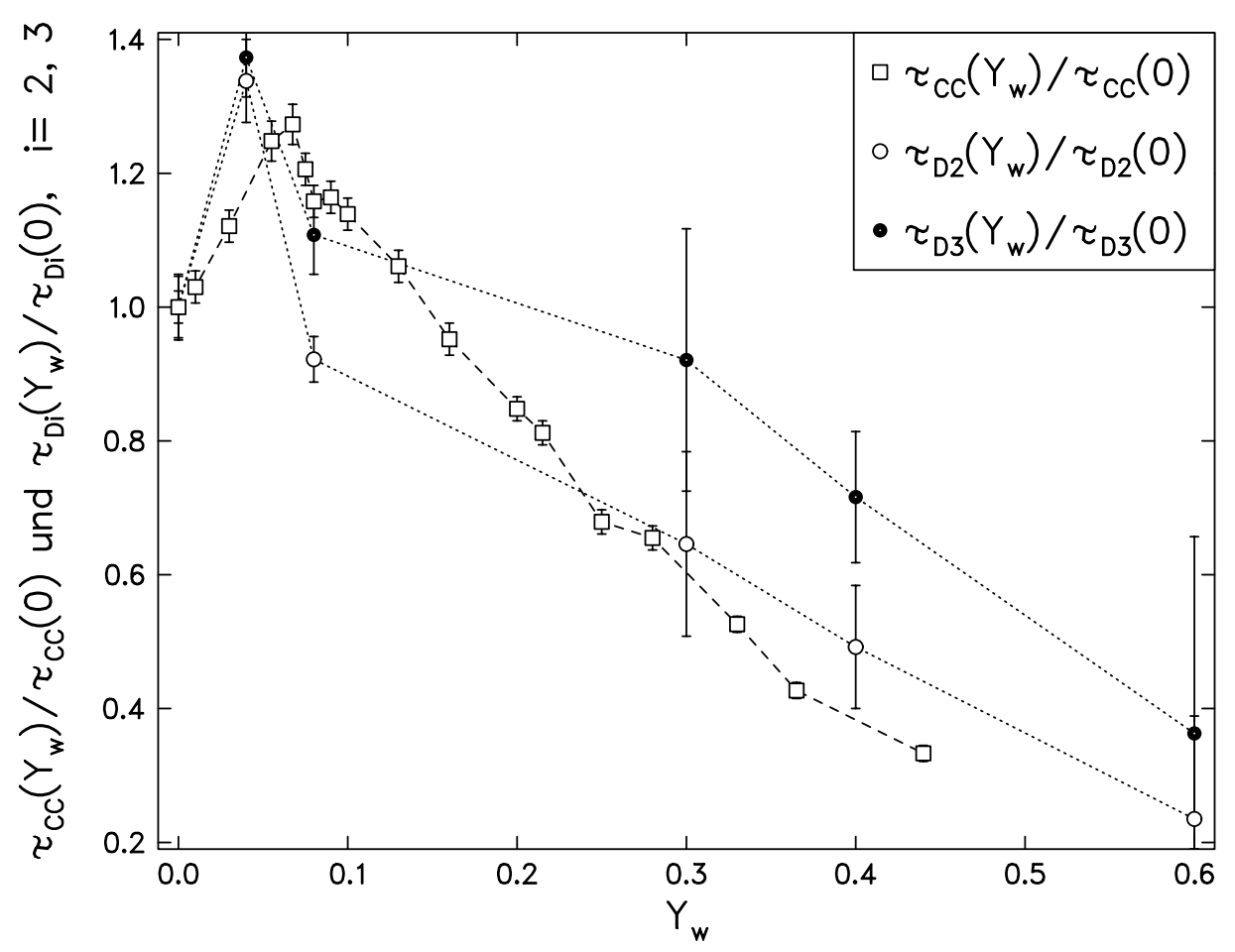

Abbildung 7.4: Auf die Relaxationszeit $\tau_{\mathrm{Di}}\left(Y_{\mathrm{W}}=0\right)$ der reinen Schmelze normierte Relaxationszeiten $\tau_{\mathrm{Di}}, i=2$, 3, nach Tab. 7.1 für die verschiedenen PEG 400/Wasser-Mischungen, dargestellt als Funktion des Massenbruchs $Y_{\mathrm{W}}$ von Wasser. Zusätzlich ist die auf $\tau_{\mathrm{CC}}\left(Y_{\mathrm{W}}=0\right)$ normierte dielektrische Cole-Cole-Relaxationszeit nach Tab. 6.2 mit eingetragen. Alle Werte für $25^{\circ} \mathrm{C}$. Gestrichelt/gepunktet: Hilfslinien.

sie treten jedoch in allen drei Einzelzeitrelaxationen auf. Ihre Existenz wird außerdem durch die Ergebnisse der dielektrischen Spektroskopie bestätigt. Denn der Trend in den Relaxationszeiten der Debye-Spektralfunktionen ähnelt stark dem konzentrationsabhängigen Verlauf der dielektrischen Hauptrelaxationszeit für die PEG 400/Wasser-Mischungen (Abb. 6.2, 7.4). Entsprechend naheliegend ist auch die Interpretation dieses Effekts: Der anfängliche Anstieg der Relaxationszeit wird auf die zunehmende Vernetzung der Polymerketten mit wachsendem Wassergehalt bzw. Donator-Akzeptor-Verhältnis zurückgeführt. Mit zunehmendem Wassergehalt wächst jedoch auch die Anzahl alternativer Bindungspartner, was nach dem Wait-und-Switch-Modell die Wahrscheinlichkeit für die Umorientierung eines Kettensegments erhöht (Abschnitt 6.2.2.2). Da die Beweglichkeit eines einzelnen Segments die Flexibilität der gesamten Polymerkette entscheidend mitbestimmt, wird sich dies auch auf die Relaxationszeiten der Bewegungsmoden auswirken und $\mathrm{zu}$ einem Absinken der Relaxationszeiten $\tau_{\mathrm{D} 2}, \tau_{\mathrm{D} 3}$ und $\tau_{\mathrm{D} 4}$ führen. 
7 Beeinflussung der Kettendynamik durch Wasserstoffbrückenassoziation 


\section{Zusammenfassung und Ausblick}

In der vorliegenden Arbeit wurden die strukturellen und molekulardynamischen Eigenschaften kurzkettiger Polyethylenglykol-Schmelzen (PEG) und ihrer Mischungen mit Wasser untersucht. Zu diesem Zweck wurden breitbandige frequenzabhängige Messungen des Schallabsorptionskoeffizienten im Bereich 0.2-2000 MHz sowie der komplexen Dielektrizitätszahl im Bereich 0.4-3000 MHz vorgenommen und durch Dichte-, Schallgeschwindigkeits- und Viskositätsmessungen ergänzt. Durch Variation des Polymerisationsgrades, der Molekül-Endgruppen, der Polymer- bzw. Wasser-Konzentration in den Mischungen und der Messtemperatur konnte mit diesen Methoden ein detaillierter Einblick in die Oligomerkettendynamik und ihre Beeinflussung durch die Assoziation der Flüssigkeitsmoleküle über Wasserstoffbrückenbindungen gewonnen werden.

Die Ultraschallabsorptionsspektren der Oligoethylenglykol-Schmelzen lassen sich durch eine mit dem Polymerisationsgrad zunehmende Anzahl von Debye-Spektralfunktionen beschreiben. Die Einzelzeitrelaxationsprozesse, die diesen Spektralfunktionen zugrunde liegen, werden auf der Grundlage des Rouse-Modells als Eigenmoden der Kettendynamik interpretiert. Aus der Kettenlängenabhängigkeit der Relaxationszeiten und den Relaxationszeitverhältnissen lassen sich die in dem jeweiligen Spektrum auftretenden Einzelzeitrelaxationen den niedrigsten ungeraden Rouse-Moden zuordnen. Für das Fehlen der geraden Moden gibt es bislang nur die Vermutung, dass diese nicht mit einer signifikanten Volumenänderung einhergehen und daher akustisch nicht detektierbar sind. Hierüber könnten molekulardynamische Simulationsrechnungen Aufschluss geben.

Bis zu einer Kettenlänge von neun monomeren Einheiten folgen die Relaxationszeiten der so zugeordneten Moden sehr gut den Vorhersagen des Rouse-Modells [6] in der Näherung für lange Ketten. Interessant ist, dass diese Näherung, anders als von Rouse berechnet, auch die Dynamik der kurzen Ketten gut beschreibt. Vermutete Ursachen für die überraschend gute Übereinstimmung sind eine im Modell nicht enthaltene Kettenlängenabhängigkeit der Reibungskonstante, die sich nur bei kleinem Polymerisationsgrad auswirkt, sowie die für kurze Ketten unzureichende Modellierung durch ein diskretes Feder-Masse-System. Bei längeren Oligomeren, insbesondere bei hochfrequenten Moden, weicht das beobachtete Verhalten der Relaxationszeiten etwas von der vorhergesagten quadratischen Abhängigkeit von der Kettenlänge ab, da die dann zusätzlich vorhandenen höheren Moden im Spektrum nicht mehr sicher aufgelöst werden können.

Aus dem experimentell ermittelten Zusammenhang zwischen Polymerisationsgrad und Anzahl auftretender Moden wurde die Länge eines Rouse-Segments abgeschätzt. Hiernach hat die kleinste, von der Bewegung der Nachbarsegmen- 
te unabhängige Untereinheit der Polymerkette eine Länge von etwa drei bis vier Kohlen- bzw. Sauerstoffatomen. Dieser Wert liegt deutlich unter der im Rahmen des Rouse-Modells zu erwartenden Segmentlänge von etwa zehn rotationsfähigen (C-C-)Bindungen, deckt sich jedoch sehr gut mit der Vorhersage des von Tobolsky, Aklonis und DuPré vorgeschlagenen Damped-Torsional-Oscillator-Modells [7, 8], für das sich eine Segmentlänge von zwei bis drei rotationsfähigen Bindungen ergibt. Dies deutet darauf hin, dass die Kettendynamik der Oligoethylenglykole eher auf Torsionsbewegungen als auf Abstandsänderungen atomarer Gruppen beruht.

Die bei PEG beobachtete Modenstruktur findet sich auch in den Ultraschallabsorptionsspektren anderer Oligomerschmelzen wieder. Die Relaxationszeiten des sehr ähnlich aufgebauten PPG 1000 fügen sich hinsichtlich ihrer Abhängigkeit von Kettenlänge und Modenordnung sehr gut in die Reihe der PEG-Schmelzen ein. Auch bei dem Dimethyletherderivat PEGDME und den flüssigen $n$-Alkanen [51] ist näherungsweise ein Anstieg der Relaxationszeit mit dem Quadrat der Segmentanzahl zu beobachten. Dabei wird der Absolutwert der Relaxationszeit durch den strukturellen Aufbau der Moleküle mitbestimmt. So ist die Modendynamik von PEG, das über Wasserstoffbrückenbindungen assoziieren kann, gegenüber dem in der Schmelze unvernetzten PEGDME verlangsamt. Auch führen bereits die veränderten stereochemischen Eigenschaften der sauerstoffhaltigen Bindungen gegenüber den reinen Kohlenwasserstoffketten der $n$-Alkane zu einer leicht erhöhten Relaxationszeit.

Ein bemerkenswertes Verhalten zeigen die Relaxationsamplituden der RouseModen. Sie nehmen für alle Schmelzen mit zunehmender Relaxationszeit ab. Dabei findet sich der vom Rouse-Modell postulierte Zusammenhang von Relaxationszeit, Modenordnung und Kettenlänge auch in einer experimentell beobachteten Korrelation von Amplitude und Modenordnung bzw. Kettenlänge wieder. Die Relaxationszeitabhängigkeit selbst sowie der Temperaturverlauf der Amplituden können durch das Rouse-Modell jedoch nicht erklärt werden. Hier ist die Entwicklung eines um die Kopplung an Schallwellen erweiterten theoretischen Modells notwendig, um ein tieferes Verständnis des Amplitudenverlaufs zu erhalten. Ein Vergleich des aus den Vorgaben des Rouse-Modells abgeschätzten Scherviskositätsanteils der Amplituden mit den experimentell ermittelten Werten weist darauf hin, dass in ein erweitertes Modell neben der Scherviskosität auch die im Rouse-Modell nicht berücksichtigte Volumenviskosität einzubeziehen ist.

Die dielektrischen Spektren der Oligoethylenglykole und ihrer Mischungen mit Wasser können durch eine Cole-Cole-Spektralfunktion beschrieben werden. Ihre Hauptrelaxationszeit lässt einen deutlichen Einfluss von Wasserstoffbrücken auf die molekulare Dynamik erkennen. Erhöht man durch Verkürzung der PEG-Moleküle oder Zugabe geringer Wassermengen die Zahl der Möglichkeiten zur Bildung von Wasserstoffbrücken, so steigt die Relaxationszeit zunächst an, was auf eine Erhöhung des Vernetzungsgrades in der Flüssigkeit zurückzuführen ist. Ab einer gewissen Konzentration von Wasserstoffbrückenbindungspartnern nimmt die Relaxationszeit jedoch wieder ab. Dieses Verhalten steht im Einklang mit dem Waitand-Switch-Modell [74-76] zur Beschreibung der Umorientierungsprozesse in asso- 
ziierenden Flüssigkeiten, wonach die dielektrische Relaxationszeit auch maßgeblich von der Anzahldichte geeigneter alternativer Bindungspartner abhängt. Diese wiederum steigt mit dem Wassergehalt und abnehmender Kettenlänge, wodurch die Rate der Umorientierungsprozesse dipolarer Gruppen nach Durchlaufen eines Minimums wieder wächst.

Ein Vergleich der dielektrischen Hauptrelaxationszeit mit den Relaxationszeiten der in den Ultraschallabsorptionsspektren beobachteten Einzelzeitrelaxationen zeigt, dass auch die Moden der Kettenbewegung ein Relaxationszeitmaximum durch Vernetzung bei kleinen Wasserkonzentrationen bzw. kleinen Kettenlängen aufweisen.

Die konzentrationsabhängigen Messungen der dielektrischen Relaxationszeit bei den PEG/Wasser-Mischungen weisen eine weitere Besonderheit auf: Hier ist abhängig von Temperatur und Polymerisationsgrad ein kleines lokales Relaxationszeitminimum bei einem Wassergehalt von etwa neun Massenprozent zu beobachten, das mit steigender Temperatur und abnehmender Kettenlänge verschwindet. Es wird vermutet, das in diesem Konzentrationsbereich eine vorkritische Entmischung in Mikrophasen mit höherem bzw. niedrigerem Wassergehalt als der mittleren Wasserkonzentration auftritt. Diese Vermutung wird durch neuere Molekulardynamiksimulationen [77] unterstützt. 
8 Zusammenfassung und Ausblick 


\section{Anhang}

\subsection{Ergänzende Dichte-, Schallgeschwindigkeits- und Viskositätsmessungen}

Zur Ergänzung und zur weiteren Auswertung der Ergebnisse aus der akustischen und der dielektrischen Spektroskopie wurden für die untersuchten Substanzen Messungen der Dichte, Schallgeschwindigkeit und Viskosität vorgenommen. Die Ergebnisse dieser Messungen werden im Folgenden tabellarisch aufgeführt.

Die Dichtemessungen wurden mit einem Hochpräzisionsdensitometer (Physica DMA 500, Anton Paar) durchgeführt, das nach dem Biegeschwingerprinzip arbeitet. Das Gerät bestimmt die Dichte nach Herstellerangaben bis auf $10^{-5} \mathrm{~g} / \mathrm{cm}^{3}$ genau. Es hat sich jedoch gezeigt, dass bei den zähflüssigen Schmelzen und den zur Blasenbildung neigenden Polymer-Wasser-Mischungen die Messwerte aufgrund von Schwankungen in der Befüllung und Benetzung der Messkapillare stärker variieren können. Auf der Basis von Wiederholungsmessungen wird daher ein Fehler von $3 \cdot 10^{-4} \mathrm{~g} / \mathrm{cm}^{3}$ angegeben. Die Schallgeschwindigkeitswerte wurden mit Hilfe des Resonatorverfahrens auf wenige Promille genau ermittelt (vergl. Abschnitt 3.2.1.3). Zur Bestimmung der Scherviskosität wurde ein Kugelfallviskosimeter (B/BH, Haake) verwendet. Der Fehler dieser Methode liegt bei etwa $1 \%$. 


\begin{tabular}{|c|c|c|c|}
\hline Substanz & $\rho\left[\mathrm{g} / \mathrm{cm}^{3}\right] \pm 0.03 \%$ & $c_{\mathrm{s}}[\mathrm{m} / \mathrm{s}] \pm 0.2 \%$ & $\eta_{\mathrm{s}}[\mathrm{mPas}] \pm 1 \%$ \\
\hline EG & 1.1100 & 1654.0 & 17.1 \\
\hline DEG & 1.1168 & - & - \\
\hline TEG & 1.1198 & 1610.2 & 37.5 \\
\hline PEG 200 & 1.1212 & 1600.8 & 50.7 \\
\hline PEG 300 & 1.1218 & 1596.0 & 70.4 \\
\hline PEG 400 & 1.1225 & 1591.9 & 93.1 \\
\hline PEG 600 & 1.1227 & 1586.1 & 133.5 \\
\hline PPG 1000 & 1.0009 & 1351.2 & 149.6 \\
\hline TEGDME & 1.0069 & 1378.9 & 3.5 \\
\hline PEGDME 250 & 1.0314 & 1423.1 & 4.9 \\
\hline PEGDME 500 & 1.0709 & 1484.8 & 20.5 \\
\hline TEGMEE & 1.0181 & - & - \\
\hline
\end{tabular}

Tabelle 9.1: Dichte $\rho$, Schallgeschwindigkeit $c_{\mathrm{s}}$ und Scherviskosität $\eta_{\mathrm{s}}$ der untersuchten OligomerSchmelzen bei $25^{\circ} \mathrm{C}$.

\begin{tabular}{lcccc}
$Y_{\mathrm{W}}$ & \multicolumn{2}{c}{$\rho\left[\mathrm{g} / \mathrm{cm}^{3}\right] \pm 0.03 \%$} & \multicolumn{2}{c}{$c_{\mathrm{S}}[\mathrm{m} / \mathrm{s}] \pm 0.2 \%$} \\
\hline & & & & \\
& $25{ }^{\circ} \mathrm{C}$ & $40{ }^{\circ} \mathrm{C}$ & $25{ }^{\circ} \mathrm{C}$ & $40^{\circ} \mathrm{C}$ \\
0 & & & & \\
0.1 & 1.0705 & 1.0574 & 1488.2 & 1431.0 \\
0.2 & 1.0764 & 1.0631 & 1555.2 & 1503.1 \\
0.3 & 1.0794 & 1.0662 & 1623.8 & 1571.2 \\
0.4 & 1.0743 & 1.06614 & 1683.2 & 1630.9 \\
0.5 & 1.0648 & 1.0531 & 1721.1 & 1670.9 \\
0.6 & 1.0552 & 1.0419 & 1709.3 & 1686.8 \\
0.7 & 1.0380 & 1.0295 & 1669.0 & 1652.2 \\
0.8 & 1.0238 & 1.0167 & 1612.6 & 1614.7 \\
0.9 & 1.0100 & 1.0042 & 1554.7 & 1571.2 \\
& & & & \\
\hline
\end{tabular}

Tabelle 9.2: Dichte und Schallgeschwindigkeit der PEGDME 500/Wasser-Mischungen bei $25^{\circ} \mathrm{C}$ und $40^{\circ} \mathrm{C} . Y_{\mathrm{W}}$ : Massenbruch von Wasser. 


\begin{tabular}{|c|c|c|c|c|c|c|c|c|c|}
\hline \multirow[t]{2}{*}{$Y_{\mathrm{W}}$} & \multicolumn{3}{|c|}{$\rho\left[\mathrm{g} / \mathrm{cm}^{3}\right] \pm 0.03 \%$} & \multicolumn{3}{|c|}{$c_{\mathrm{s}}[\mathrm{m} / \mathrm{s}] \pm 0.2 \%$} & \multicolumn{3}{|c|}{$\eta_{\mathrm{s}}[\mathrm{mPas}] \pm 1 \%$} \\
\hline & $10^{\circ} \mathrm{C}$ & $25^{\circ} \mathrm{C}$ & $40^{\circ} \mathrm{C}$ & $10^{\circ} \mathrm{C}$ & $25^{\circ} \mathrm{C}$ & $40^{\circ} \mathrm{C}$ & $10^{\circ} \mathrm{C}$ & $25^{\circ} \mathrm{C}$ & $40^{\circ} \mathrm{C}$ \\
\hline 0 & 1.1351 & 1.1225 & 1.1100 & 1642.4 & 1590.4 & 1541.4 & 229.8 & 92.7 & 45.8 \\
\hline 0.01 & 1.1351 & 1.1224 & 1.1099 & 1646.5 & 1592.3 & 1546.6 & 231.2 & 92.5 & 45.3 \\
\hline 0.03 & 1.1348 & 1.1222 & 1.1097 & 1654.8 & 1604.3 & 1556.1 & 237.8 & 91.8 & 43.8 \\
\hline 0.055 & 1.1343 & 1.1218 & 1.1093 & 1667.5 & 1617.7 & 1570.3 & 234.6 & 86.1 & 42.1 \\
\hline 0.065 & 1.1342 & 1.1216 & 1.1092 & 1672.8 & 1623.8 & 1576.5 & - & - & - \\
\hline 0.0675 & 1.1340 & 1.1215 & 1.1091 & 1675.2 & 1625.2 & 1578.5 & - & - & - \\
\hline 0.075 & 1.1340 & 1.1214 & 1.1090 & 1678.7 & 1629.8 & 1580.6 & - & - & - \\
\hline 0.8 & 1.1339 & 1.1214 & 1.1089 & 1680.9 & 1631.7 & 1584.7 & 223.2 & 83.6 & 39.4 \\
\hline 0.1 & 1.1334 & 1.1208 & 1.1084 & 1692.7 & 1644.6 & 1597.2 & 231.2 & 92.5 & 45.3 \\
\hline 0.13 & 1.1325 & 1.1199 & 1.1074 & 1709.2 & 1660.8 & 1613.8 & 194.6 & 72.2 & 33.9 \\
\hline 0.2 & 1.1292 & 1.1166 & 1.1041 & 1746.9 & 1698.5 & 1651.5 & 234.78 & 92.4 & 44.7 \\
\hline 0.215 & 1.1283 & 1.1157 & 1.1033 & 1754.4 & 1706.5 & 1659.1 & - & - & - \\
\hline 0.23 & 1.1273 & 1.1148 & 1.1023 & 1761.0 & 1712.8 & 1665.9 & 141.7 & 54.5 & 26.2 \\
\hline 0.25 & 1.1257 & 1.1132 & 1.1008 & 1768.4 & 1720.7 & 1673.5 & - & - & - \\
\hline 0.31 & 1.1200 & 1.1078 & 1.0955 & 1787.9 & 1741.3 & 1695.7 & - & - & - \\
\hline 0.365 & 1.1132 & 1.1015 & 1.0896 & 1794.7 & 1751.3 & 1706.9 & 52.8 & 23.2 & 12.2 \\
\hline 0.44 & 1.1024 & 1.0877 & 1.0801 & 1791.8 & 1753.1 & 1713.4 & - & - & - \\
\hline 0.6 & 1.0733 & 1.0649 & 1.0556 & 1728.9 & 1710.2 & 1690.4 & - & - & - \\
\hline 0.8 & 1.0350 & 1.0298 & 1.0231 & 1591.8 & 1607.8 & 1614.1 & - & - & - \\
\hline
\end{tabular}

Tabelle 9.3: Dichte $\rho$ und Schallgeschwindigkeit $c_{\mathrm{s}}$ und Viskosität $\eta_{\mathrm{s}}$ der PEG 400/WasserMischungen bei $10^{\circ} \mathrm{C}, 25^{\circ} \mathrm{C}$ und $40^{\circ} \mathrm{C} . Y_{\mathrm{W}}$ : Massenbruch von Wasser. 
9 Anhang 


\section{Literaturverzeichnis}

[1] Harris J.M. und Zalipsky S. (Herausgeber), Poly(ethylene glycol) - Chemistry and Biological Applications, Band 680 von ACS Symposium Series, American Chemical Society, Washington, DC (1997).

[2] Seifert B., Minanetzis G., Groth T., Albrecht W., Richau K., Missirlis Y., Paul D. und von Sengbusch G., Artificial Organs 26, 189 (2002).

[3] Eckert S., Hoffmann S., G.Meier und Alig I., Phys. Chem. Chem. Phys. 4, 2594 (2002).

[4] Bae J.R., Kim J.K. und Yi M.H., Jpn. J. Appl. Phys. 39, 2946 (2000).

[5] Fenner D.B., J. Chem. Phys. 87, 4 (1987).

[6] Rouse, JR. P.E., J. Chem. Phys. 21(7), 1272 (1953).

[7] Tobolsky A.V. und DuPré D.B., Adv. Polymer Sci. 6, 103 (1969).

[8] Tobolsky A.V. und Aklonis J.J., J. Phys. Chem. 68, 1970 (1964).

[9] Clariant Functional Chemicals, Polyalkylene/Polyethylene Glycols, Produktinformation (2003).

[10] Bailey F.E. und Koleske J.V., Poly(ethylene oxid), Academic Press, New York (1976).

[11] Branca C., Faraone A., Magazú S., Maisano G., Magliardo P. und Villari V., J. Mol. Liquids 87, 21 (2000).

[12] Bird R.B., Armstrong R.C. und Hassager O., Fluid Mechanics, Band 1 von Dynamics of Polymeric Liquids, John Wiley \& Sons (1987).

[13] www.de.wikipedia.org/wiki/Polyethylenglykol.

[14] KJellander R. und Florin E., J. Chem. Soc., Faraday Trans. 1 77, 2053 (1981).

[15] Bae Y.C., Shim J.J., Soane D.S. und Prausnitz J.M., J. Appl. Polym. Sci. 47, 1193 (1993). 
[16] Saeki S., Kuwahara N., Nakata M. und Kaneko M., Polymer 17, 685 (1976).

[17] Crupi V., Janelli M.P., Magazù S., Maisano G., Majolino D., MigLiardo P. und Ponterio R., J. Mol. Struct. 381, 207 (1996).

[18] Źwirbla W., Sikorska A. und Linde B.B.J., J. Mol. Struct. 743, 49 (2005).

[19] Branca C., Magazì S., Maisano G., Migliardo F., Migliardo P. und G.Romeo, J. Phys. Chem. B 106, 10272 (2002).

[20] Bhatia A.B., Ultrasonic Absorption, Oxford University Press, Oxford (1967).

[21] Landau L.D. und Lifschitz E.M., Hydrodynamik, Band 6 von Lehrbuch der Theoretischen Physik, Akademie-Verlag, Berlin (1966).

[22] Telgmann T., Dissertation, Math.-Nat. Fak. Univ. Göttingen (1997).

[23] Kühnel V., Dissertation, Math.-Nat. Fak. Univ. Göttingen (1995).

[24] Behrends R. und KaAtze U., J. Phys. Chem. A 105, 5829 (2001).

[25] Slutsky L.J., in: Ultrasonics, herausgegeben von L. Marton und C. Marton, Band 19 von Methods of Experimental Physics, Academic Press, New York (1981).

[26] Strehlow H., Rapid Reactions in Solutions, VCH Verlagsgesellschaft, Weinheim (1992).

[27] Schrader W., Dissertation, Math.-Nat. Fak. Univ. Göttingen (2001).

[28] Kaatze U., Behrends R. und Lautscham K., Ultrasonics 39, 393 (2001).

[29] Labhardt A. und Schwarz G., Ber. Bunsenges. Phys. Chem. 80(1), 83 (1976).

[30] Labhardt A., Dissertation, Math.-Nat. Fak. Univ. Basel (1975).

[31] Hagen R., Dissertation, Math.-Nat. Fak. Univ. Göttingen (2003).

[32] Kononenko V.S., Sov. Phys. Acoust. 31, 499 (1985).

[33] Behrends R., Diplomarbeit, Math.-Nat. Fak. Univ. Göttingen (1995).

[34] Telgmann T., Diplomarbeit, Math.-Nat. Fak. Univ. Göttingen (1994).

[35] Polacek R., Dissertation, Math.-Nat. Fak. Univ. Göttingen (2003). 
[36] Hagen R., Diplomarbeit, Math.-Nat. Fak. Univ. Göttingen (1998).

[37] Rupprecht A., Dissertation, Math.-Nat. Fak. Univ. Göttingen (1997).

[38] FAY B., Acustica 36, 209 (1976/1977).

[39] Menzel K., Dissertation, Math.-Nat. Fak. Univ. Göttingen (1993).

[40] Kaatze U. und Lautscham K., J. Phys. E: Sci.Instrum. 19, 1046 (1986).

[41] Gallus T., Dissertation, Math.-Nat. Fak. Univ. Göttingen (1996).

[42] Kaatze U., Lautscham K., Menzel K. und Schwerdtfeger S., Meas. Sci. Technol. 4, 1257 (1993).

[43] Kaatze U., Lautscham K. und Brai M., J. Phys. E: Sci. Instrum. 21, 98 (1988).

[44] Kaatze U., Kuehnel V. und Weiss G., Ultrasonics 34, 51 (1996).

[45] Bömmel H.E. und Dransfeld K., Phys. Rew. Lett. 1, 234 (1958).

[46] Brai M. und Kaatze U., J. Phys. Chem. 96, 8946 (1992).

[47] Marquardt D.W., J. Soc. Indust. Appl. Math. 2, 2 (1963).

[48] Strobl G., The Physics of Polymers, Springer, Berlin (1997).

[49] Boal D., Mechanics of the Cell, Cambridge University Press (2002).

[50] FröHlich H., Theory of Dielectrics. Dielectric Constant and Dielectric Loss, Clarendon Press, Oxford (1958).

[51] Behrends R. und Kaatze U., J. Phys. Chem. A 104(15), 3269 (2000).

[52] Viswanath D.S. und Natarajan G., Data Book on the Viscosity of Liquids, Hemisphere Publishing Corporation, New York (1989).

[53] Eliel E.L. und Wilen S.H., Stereochemistry of Organic Compounds, John Wiley \& Sons, New York (1994).

[54] Lamb J., in: Physical Acoustics, herausgegeben von W.P. Mason, Band 2, Academic Press, New York (1965).

[55] Kantze U., Hushcha T.O. und Eggers F., J. Sol. Chem. 29(4), 299 (2000).

[56] Schulz M. und Alig I., J. Chem. Phys. 97, 2772 (1992).

[57] Alig I., Stieber F., Bakhramov A.D., Manucharov Y.S. und SoloVYeV V.A., Polymer 30, 842 (1989). 
[58] Solovyev V.A., Manucharow Y.S. und Alig I., Acta Polymerica 40(8), 513 (1989).

[59] Alig I., Stieber F., Bakhramov A.D., Manucharov Y.S., ManuchaRova S.A. und Solovyev V.A., Polymer 31, 877 (1990).

[60] Saito N., Okano K., Imayanagi S. und Hideshima T., Solid State Physics, Band 14, Academic Press, New York (1963).

[61] Grigor'ev I.A.S.B., Manucharov Y.S. und Manucharova S.A., Acta Polymerica 37(1986), 698 (1985).

[62] Hill N.E., Vaughan W.E., Price A.H. und Davies M., Dielectric Properties and Molecular Behaviour, The Van Nostrand Series in Physical Chemistry LTD, Van Nostrand Reinhold Company, London (1969).

[63] Cole K.S. und Cole R.H., J. Chem. Phys. 9, 341 (1941).

[64] Böttcher C.J. und BordewiJk P., Dielectrics in time-dependent fields, Band 2 von Theory of Electric Polarization, Elsevier Scientific Publishing Company, Amsterdam (1978).

[65] Schrader W., Diplomarbeit, Math.-Nat. Fak. Univ. Göttingen (1998).

[66] Gabriel B., Diplomarbeit, Math.-Nat. Fak. Univ. Göttingen (1992).

[67] Menzel K., Diplomarbeit, Math.-Nat. Fak. Univ. Göttingen (1990).

[68] Göttmann O., Kantze U. und Petong P., Meas. Sci. Technol. 7, 525 (1996).

[69] Meyer E. und Pottel R., Physikalische Grundlagen der Hochfrequenztechnik, Vieweg (1969).

[70] Hanke E., von Roden K. und Kantze U., J. Chem. Phys. 125(084507) (2006).

[71] Kaatze U., Lönnecke-Gabel V. und Pottel R., Z. Phys. Chem. 175, 165 (1992).

[72] Kantze U., J. Chem. Eng. Data 37, 371 (1989).

[73] Sato T., Niwa H., Chiba A. und Nozaki R., J. Chem. Phys. 108, 4138 (1998).

[74] Kaftze U., Behrends R. und Pottel R., J. Non-Cryst. Solids 305, 19 (2002).

[75] Ohmine I., Tanaka H. und Wolynes P.G., J. Chem. Phys. 89, 5852 (1988). 
[76] Sciortino F., Geiger A. und Stanley H.E., J. Chem. Phys. 96, 3857 (1992).

[77] Borodin O., Bedrov D. und Smith G.D., J. Phys. Chem. B 106, 5194 (2002).

[78] Kaatze U., Ber. Bunsenges. Phys. Chem. 82, 690 (1978).

[79] Kirkwood J.G., J. Chem. Phys. 7, 911 (1939).

[80] HiLl N.E., J. Phys. C 3, 238 (1970).

[81] Maryott A.A. und Buckley F., Table of Dielectric Constants and Electric Dipole Moments of substances in the Gaseous State, Circ. No. 537, Nat. Bur. Stand. (U. S.), U. S. GPO, Washington D. C. (1953).

[82] www.dow.com/ethyleneglycol/about/properties.htm.

[83] Abboud J.L.M. und Notario R., Pure Appl. Chem. 71, 645 (1999).

[84] Hasted J.B., Aqueous Dielectrics, Chapman and Hall, London (1973). 
9 Literaturverzeichnis

128 


\section{Danksagung}

Zum Abschluss möchte ich denjenigen meinen Dank aussprechen, die mich bei der Anfertigung dieser Arbeit unterstützt haben. Herrn Prof. Dr. Dirk Ronneberger danke ich herzlich für die Betreuung meiner Arbeit. Seine ständige Gesprächsbereitschaft war mir eine große Hilfe. Herrn Priv.-Doz. Dr. Christian Jooß danke ich für das Interesse an meiner Arbeit und für die Übernahme des Korreferats. Besonders danken möchte ich Herrn Dr. Udo Kaatze für die freundschaftliche Arbeitsatmosphäre und sein stets offenes Ohr. Aus den zahlreichen Diskussionen und Gesprächen habe ich fachlich und menschlich viel dazugelernt. Herrn Dr. Ralph Behrends und Herrn Dr. Ralf Hagen danke ich sehr für die vielen konstruktiven Diskussionen, Ratschläge und Hinweise. Kerstin von Roden und Ulrike Schulz, Barbara Eberhardt, Karola Fritz und David Gottschalt gilt mein Dank für die sorgfältige Durchführung der dielektrischen Messungen, der Schallgeschwindigkeits-, Dichte- und Viskositätsmessungen. Allen Mitarbeitern der Feinmechanischen und Elektronischen Werkstätten des Dritten Physikalischen Instituts danke ich für ihre große Hilfsbereitschaft bei der Wartung und Reparatur der Messapparaturen und Geräte. Herzlich danken möchte ich außerdem Gisa Kirschmann-Schröder für ihre Unterstützung bei der Erstellung mancher Grafik sowie Thomas Geiling für seine freundliche und hilfsbereite Art, Computerprobleme aus dem Weg zu räumen. Ein großes Dankeschön geht aber auch an alle anderen Freunde und Kollegen im Dritten Physikalischen Institut für die herzliche Atmosphäre. Nicht zuletzt möchte ich meinem Freund Christian Wald danken, der mich während der gesamten Doktorarbeit in jeder Hinsicht unterstützt hat. 
Danksagung 


\section{Curriculum vitae}

Elke Susanne Hanke

Hauptstraße 14a

D-37083 Göttingen

Geburtsdatum: 4. August 1975

Geburtsort: Hildesheim

Staatsangehörigkeit: deutsch

1982-1986

1986-1995

$05 / 1995$

$10 / 1995$

$10 / 1997$

$02 / 1998$

02/1999-07/1999

01/2001-06/2001

$12 / 2001$

02/2002-09/2002

seit $10 / 2002$

seit 10/2002
Besuch der Grundschule Holle

Besuch des Gymnasiums Andreanum Hildesheim

Abitur am Gymnasium Andreanum Hildesheim

Beginn des Studiums der Physik und Mathematik an der Universität Göttingen. Studienziel: Erste Staatsprüfung für das Lehramt an Gymnasien

Zwischenprüfung in Mathematik an der Universität Göttingen Zwischenprüfung in Physik an der Universität Göttingen

Auslandsstudium an der Universität Autónoma in Madrid mit einem Stipendium des Erasmus-Programms

Examensarbeit mit dem Thema „Zum Einfluss ungesättigter Lipide auf das Domänenverhalten von Membranen. Breitbandige Ultraschallspektroskopie, Schallgeschwindigkeits- und Wärmekapazitätsmessungen“, betreut durch Dr. Udo Kaatze und Prof. Dr. Dirk Ronneberger am Dritten Physikalischen Institut der Universität Göttingen

Erste Staatsprüfung für das Lehramt an Gymnasien in Physik und Mathematik an der Universität Göttingen

Stipendium des Graduiertenkollegs 723 „Raumzeitliche Signalprozesse in Neuronen und zelluläre Biophysik", Forschungsthema: „Fluktuationen von Domänen auf Membranen“

Wissenschaftliche Mitarbeiterin am Dritten Physikalischen Institut der Universität Göttingen

Doktorarbeit zum Thema „Struktureigenschaften und molekulare Dynamik flüssiger Ethylenglykol-Oligomere und ihrer Mischungen mit Wasser", betreut durch Dr. Udo Kaatze und Prof. Dr. Dirk Ronneberger am Dritten Physikalischen Institut der Universität Göttingen

Göttingen, 24. Mai 2007 